Florida International University

FIU Digital Commons

FIU Electronic Theses and Dissertations

University Graduate School

$3-1-2016$

\title{
The Influence of the Proximal Thiolate Ligand and Hydrogen Bond Network of the Proximal Helix on the Structural and Biochemical Properties of Chloroperoxidase
}

Elena Shersher

Florida International University, esher002@fiu.edu

DOI: 10.25148 /etd.FIDC000241

Follow this and additional works at: https://digitalcommons.fiu.edu/etd

Part of the Biochemistry Commons, and the Biotechnology Commons

\section{Recommended Citation}

Shersher, Elena, "The Influence of the Proximal Thiolate Ligand and Hydrogen Bond Network of the Proximal Helix on the Structural and Biochemical Properties of Chloroperoxidase" (2016). FIU Electronic Theses and Dissertations. 2483.

https://digitalcommons.fiu.edu/etd/2483 


\title{
FLORIDA INTERNATIONAL UNIVERSITY
}

Miami, Florida

\section{THE INFLUENCE OF THE PROXIMAL THIOLATE LIGAND AND HYDROGEN BOND NETWORK OF THE PROXIMAL HELIX ON THE STRUCTURAL AND BIOCHEMICAL PROPERTIES OF CHLOROPEROXIDASE}

A dissertation submitted in partial fulfillment of

\author{
the requirements for the degree of \\ DOCTOR OF PHILOSOPHY \\ in \\ BIOCHEMISTRY \\ by
}

Elena Shersher 
To: Dean Michael R. Heithaus

College of Arts, Sciences, and Education

This dissertation, written by Elena Shersher, and entitled The Influence of the Proximal Thiolate Ligand and Hydrogen Bond Network of the Proximal Helix on the Structural and Biochemical Properties of Chloroperoxidase, having been approved in respect to style and intellectual content, is referred to you for judgment.

We have read this dissertation and recommend that it be approved.

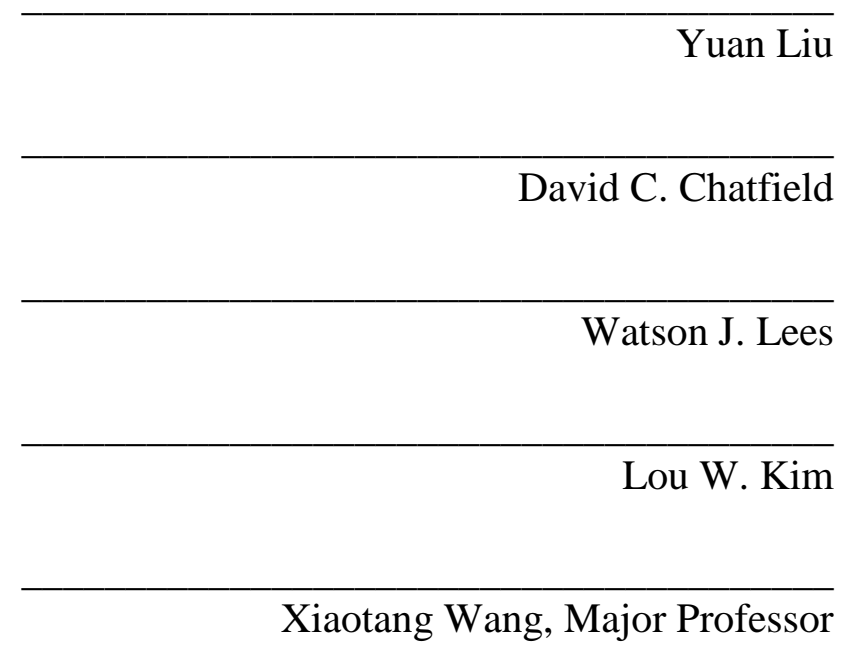

Date of Defense: March 1, 2016

The dissertation of Elena Shersher is approved.

Dean Michael R. Heithaus College of Arts, Sciences and Education

Andrés G. Gil Vice President for Research and Economic Development and Dean of the University Graduate School

Florida International University, 2016 
(C) Copyright 2016 by Elena Shersher

All rights reserved. 


\section{DEDICATION}

I dedicate this dissertation to my husband Vadim and my daughter Angelina. Their

love, understanding, encouragement, and support made completion of this work possible. 


\section{ACKNOWLEDGMENTS}

Being a part of graduate community at FIU has been a wonderful and rewarding experience. In the past few years with the help of my family, professors, colleagues, and friends, I have grown so much both professionally and personally. First of all, I wish to express my sincerest and deepest gratitude to my major professor, Dr. Xiaotang Wang, for his incredible support, encouragement, guidance, and patience. He was always there for me during both good and tough times in my work. He provided me with sufficient freedom in choosing and carrying out research projects that helped me become confident in my research and taught me to enjoy a good challenge. Dr. Wang is a wonderful advisor and a generous, understanding, caring, and kind person. I have been very lucky to have had him as my major professor.

I would also like to thank all my committee members, Dr. David Chatfield, Dr. Yuan Liu, Dr. Watson Lees, and Dr. Leung Kim, for their valuable time, professional advice and assistance. I want to acknowledge and thank my research teammates and friends, Elwood Kwong, Qinghao He, and Yongjian Guo, for all of the insightful discussions, kind help, and providing a pleasant research environment. They always made me feel like our research team is our research family. Especially I would like to thank Armando Pardillo from Dr. Chatfield's lab for inspiring discussions about CPO, for teaching me how to use docking and graphics software for my research, and helpful suggestions. I want to express my gratitude to all of my family and friends for encouraging and believing in me.

Finally, I would like to acknowledge the Department of Chemistry and Biochemistry, Florida International University and the National Institutes of Health of General Medical 
Sciences, for the financial support through Presidential Scholarship and Biomedical Research Initiative FIU Student Summer Research Awards. 
ABSTRACT OF THE DISSERTATION

THE INFLUENCE OF THE PROXIMAL THIOLATE LIGAND AND HYDROGEN

BOND NETWORK OF THE PROXIMAL HELIX ON THE STRUCTURAL AND

BIOCHEMICAL PROPERTIES OF CHLOROPEROXIDASE

by

Elena Shersher

Florida International University, 2016

Miami, Florida

Professor Xiaotang Wang, Major Professor

Chloroperoxidase (CPO) from Caldariomyces fumago is a versatile heme enzyme with great potential for environmental and pharmaceutical applications. It catalyzes a plethora of reactions including halogenation, dismutation, epoxidation, and oxidation. The diverse catalytic capabilities of CPO have long been attributed to the protein's distinct active site that combines structural features of peroxidases and cytochromes P450. Particularly, the role of the axial thiolate ligand in CPO catalysis has been much debated. Furthermore, no data are available on the role of hydrogen bonding between Arg26 Asn37 and Ala27 - Asn33 of the proximal helix in defining the structural and catalytic properties of CPO.

In order to investigate the influence of the proximal thiolate and the proximal hydrogen bond network on the structural and biochemical properties of CPO, several mutant CPOs were constructed and characterized using various spectroscopic techniques and enzymatic assays. Cysteine 29, which coordinates to the heme, was replaced with a His (C29H) to mimic the proximal ligation of classical peroxidases. The UV-Vis spectrum 
of the carbon monoxide complex of ferrous $\mathrm{C} 29 \mathrm{H}$ mutant remained essentially identical to that of wild type (WT) CPO and P450 although the ferric state of the variant enzyme showed a spectral pattern reminiscent of a classical histidine ligated heme peroxidase. Histidine ligation was further confirmed by paramagnetic NMR spectroscopy. Contrary to a previous report, the specific chlorination activity of $\mathrm{C} 29 \mathrm{H}$ was essentially abolished (less than $1 \%$ of that of WT CPO) but the epoxidation and peroxidation activities were enhanced 10-fold and 55-fold, respectively. These findings demonstrate for the first time that the heme ligand, Cys29 in CPO, is not a prerequisite for CPO's unique P450-like spectroscopic signatures but is constitutive for the protein's versatile catalytic activities.

Arginine 26 and Asparagine 33 in the proximal heme pocket were replaced with Ala (R26A, N33A, and R26A/N33A) to disrupt hydrogen bonding. Tertiary structures and heme environments of R26A, N33A, and R26A/N33A differed from those of WT CPO as determined by CD spectroscopy. The specific chlorination and dismutation activities of all mutants were almost abolished but the peroxidation and epoxidation rates were increased. These results show that the proximal hydrogen bond network plays an important role in maintaining the structure and catalytic diversity of CPO. 


\section{TABLE OF CONTENTS}

CHAPTER

PAGE

I. HEME PROTEIN DIVERSITY: STRUCTURE, FUNCTION, APPLICATIONS ........1

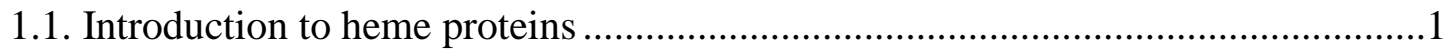

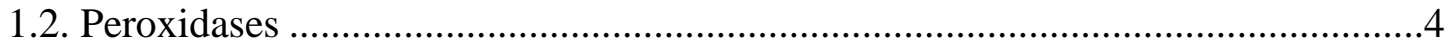

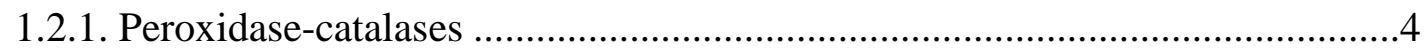

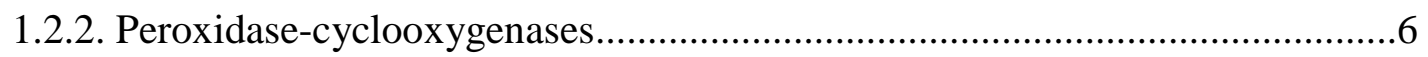

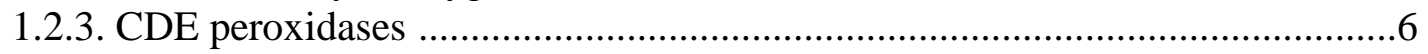

1.2.4. Peroxidase-peroxygenases ………………….......................................

1.3. Chloroperoxidase ........................................................................................10

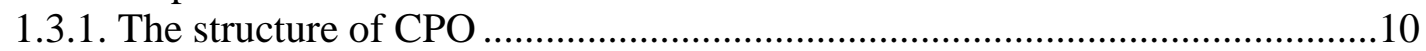

1.3.2. Overview of $\mathrm{CPO}$ catalytic cycle.................................................................

1.3.2.1. Active intermediates: Compound I, Compound II, and Compound X ..15

1.3.3. Reactions catalyzed by CPO ....................................................................17

1.3.3.1. One-electron oxidations ...................................................................17

1.3.3.2. Two-electron oxidations ....................................................................21

1.3.4. Ligand-binding properties.............................................................................30

1.4. Spectroscopic properties of heme proteins …………………………..................31

1.5. Site-directed mutagenesis to study structure-function relationships in heme

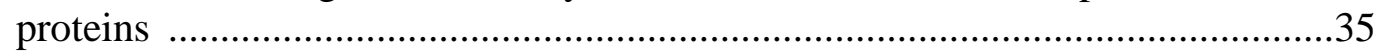

II. THE ROLE OF THE PROXIMAL LIGAND IN CPO, A LONG LASTING

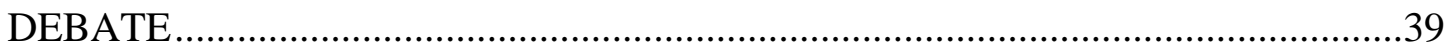

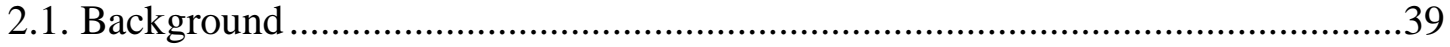

2.2. Experimental procedures .........................................................................

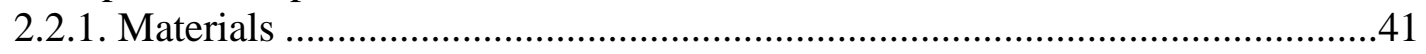

2.2.2. Construction of $\mathrm{C} 29 \mathrm{H}$ CPO gene and plasmid propagation .............................41

2.2.3. Transformation of Aspergillus niger with $\mathrm{C} 29 \mathrm{H} \mathrm{pCPO}$ and mutant

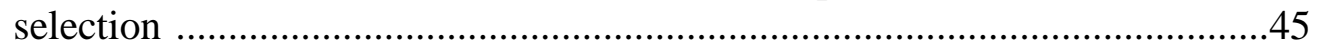

2.2.4. Expression and purification of C29H CPO ……………..............................4

2.2.5. Spectroscopic characterization of C29H CPO ............................................4

2.2.5.1. Proton nuclear magnetic resonance spectroscopy ……………………...47

2.2.5.2. Circular dichroism spectroscopy ..........................................................48

2.2.5.3. UV-Visible spectrophotometry ...........................................................49

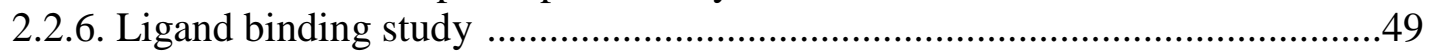

2.2.7. Enzymatic activity assays ...........................................................................49

2.2.7.1. Peroxidation assay (ABTS) ………………………………………....50

2.2.7.2. Chlorination assay (MCD) ……………………...............................50

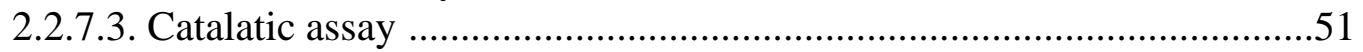

2.2.7.4. Epoxidation assay (styrene) ………………………..............................51

2.2.7.5. Calculations of specific activities ........................................................51

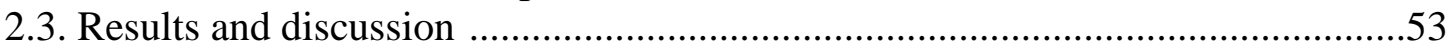

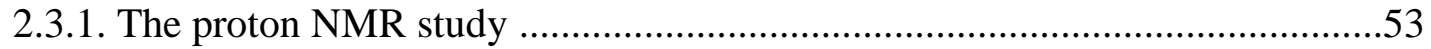


2.3.2. Circular dichroism of WT and variant CPO .................................................54

2.3.3. UV-Visible spectroscopic properties of WT and C29H CPO ..........................55

2.3.4. CO binding to WT, C29H, C29H/C79H, and C29H/C79H/C87H CPO ........59

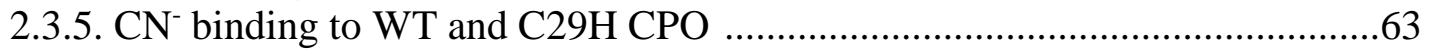

2.3.6. Catalytic properties of WT and C29H CPO ..................................................65

2.3.7. $\mathrm{pH}$ profiles of catalytic activities of WT and C29H CPO ..............................69

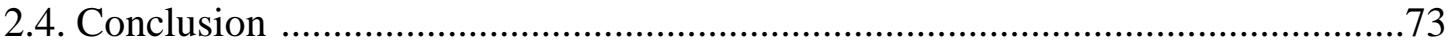

III. EFFECTS OF HYDROGEN BOND NETWORK IN THE PROXIMAL HELIX

ON THE SPECTROSCOPIC PROPERTIES OF CHLOROPEROXIDASE ...............74

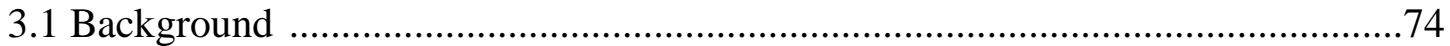

3.2. Experimental procedures ...............................................................................

3.2.1. Construction of R26A,N33A, and R26A/N33A CPO genes ...........................77

3.2.2. Transformation of A. niger with mutant $\mathrm{pCPO}$ and purification of R26A, N33A, and R26A/N33A CPO ……….....................................................79

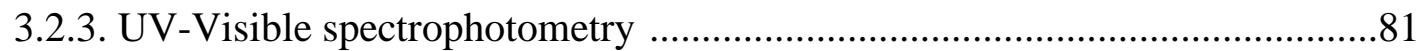

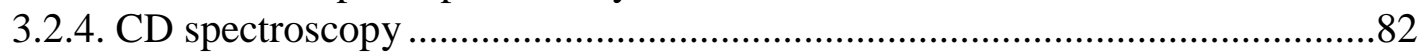

3.3. Results and discussion .......................................................................................82

3.3.1. Secondary and tertiary CD spectroscopy of WT and mutant CPO..................82

3.3.2. UV-Vis spectroscopic properties of WT, R26A, N33A, and

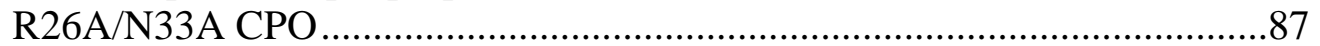

3.3.3. Ligand binding to WT, R26A, N33A, and R26A/N33A CPO ......................92

3.3.3.1. CO binding to WT and mutant CPO....................................................93

3.3.3.2. Cyanide binding to WT and mutant CPO ..............................................96

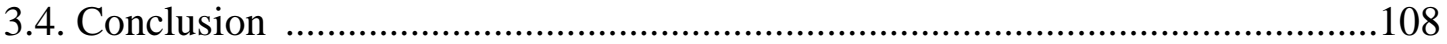

IV. EFFECTS OF HYDROGEN BOND NETWORK IN THE PROXIMAL HELIX

ON CATALYTIC PROPERTIES OF CHLOROPEROXIDASE................................109

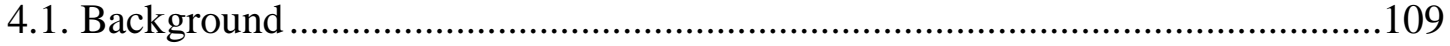

4.2. Experimental procedures ……………………….....................................113

4.2.1. Construction of R26A, N33A, and R26A/N33A CPO mutants ....................113

4.2.3. Enzymatic assays ........................................................................................113

4.3. Results and discussion ......................................................................................114

4.3.1. Chlorination and dismutation activities of WT and mutant $\mathrm{CPO}$ at various $\mathrm{pH}$

4.3.2. Peroxidation and epoxidation activities of $\mathrm{WT}$ and mutant $\mathrm{CPO}$ at various

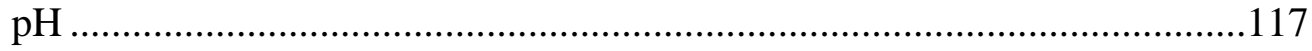

4.3.3. Comparison of $\mathrm{WT}$ and mutant $\mathrm{CPO}$ activities at optimal $\mathrm{pH}$....................120

4.4. Conclusion ......................................................................................................123

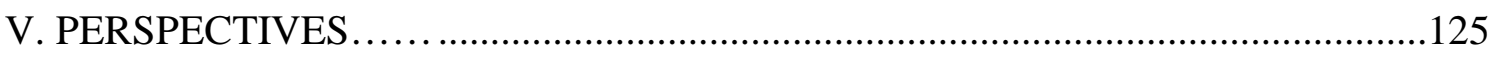

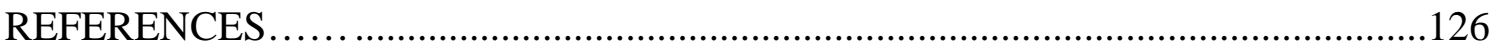

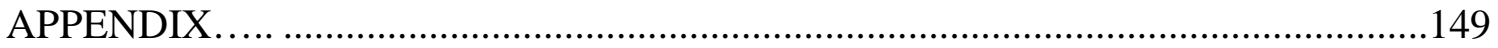




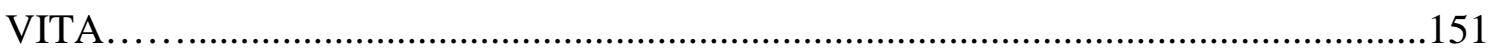




\section{LIST OF TABLES}

TABLE

PAGE

1.1 Heme proteins and their biological functions. ...............................................

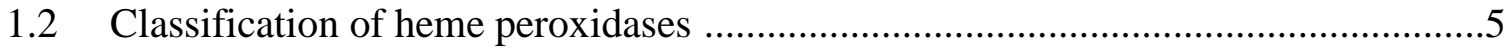

1.3 Effects of distal and proximal active site mutations on catalytic activities of some

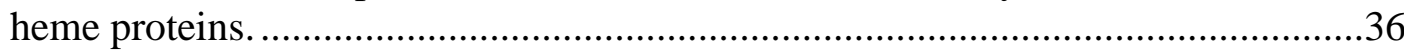

2.1 Effects of proximal active site mutations on catalytic activities of some

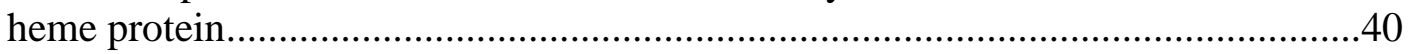

2.2 Reagents used in the PCR reaction. .............................................................43

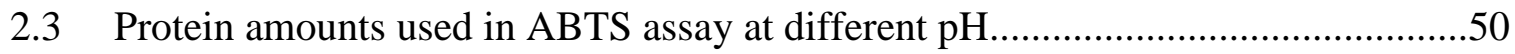

2.4 Extinction coefficients of oxidized ABTS, MCD, hydrogen peroxide, and styrene..

2.5 UV-Vis spectral properties of ferric WT and C29H CPO in phosphate buffer, pH 5.9

2.6 UV-Vis spectral properties of ferric WT and C29H CPO and their cyanide complexes at $\mathrm{pH}$ 5.9.

3.1 CD spectral properties of ferric WT and mutant CPO in phosphate buffer, pH 5.9 in the heme region $((+),(-),(s)$ indicate positive and negative signals, and a shoulder in a band, respectively).

3.2 UV-Vis spectral properties of ferric WT and mutant CPO at pH 5.9 (s-shoulder, n.s.- no shoulder, s.s.- slight shoulder)

3.3 Spectral properties of ferrous WT, R26A, N33A, and R26A/N33A CPO and their CO complexes.

3.4 UV-Vis spectral properties of ferric WT and mutant CPO and their cyanide complexes at $\mathrm{pH} 6.0$ and $\mathrm{pH} 12.0$

4.1 Enzyme amounts used in ABTS assay at different $\mathrm{pH}$ 


\section{LIST OF FIGURES}

FIGURE

PAGE

1.1 Structures of heme groups most frequently found in heme proteins ......................3

1.2 Active site structures of cytochrome c peroxidase from $S$. cerevisiae (A), lignin peroxidase from $P$. chrysosporium (B), horseradish peroxidase from A. rusticana (C), lactoperoxidase from $B$. bubalis (D), dye decolorizing peroxidase from $T$. cucumeris $(\mathrm{E})$, and chloroperoxidase from $C$. fumago $(\mathrm{F})$.........7

1.3 Active site structures of aromatic peroxygenase from $A$. aegerita (A), chloroperoxidase from C. fumago (B), and cytochrome P450 3A4 from Homo sapiens (C).

1.4 Crystal structure of chloroperoxidase from $C$. fumago .....................................11

1.5 Slice through surface representation of CPO .................................................13

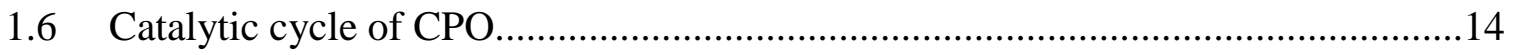

1.7 Mechanism of CPO Compound I formation, the horizontal bold line

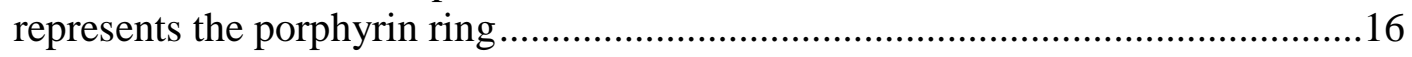

1.8 CPO-catalyzed chlorination mechanism .....................................................17

1.9 Mechanistic routes of different types of reactions catalyzed by CPO:

(a) halogenation, (b) phenol oxidation, (c) sulfoxidation

1.10 Oxidation of ABTS by CPO using hydrogen peroxide as an oxidant .20

1.11 Dehalogenation of (a) trihalophenol, where $\mathrm{X}=\mathrm{F}^{-}, \mathrm{Cl}^{-}, \mathrm{Br}^{-}, \mathrm{I}^{-}$and (b) 4-fluorophenol.

1.12 Examples of CPO-catalyzed halogenations : (a) 1,3-cyclopentanedione, (b) phenol, (c) alkene, (d) anthracene (aromatic hydrocarbon).

1.13 Possible transition states in epoxidation of cis-2-methylstyrene .....

1.14 Stereoselective epoxidation of (a) cis-heptene and (b) 3-methyl-3butenoate with yield and enantiomeric excess (ee) percentages.

1.15 Stereoselective sulfoxidation of (a,b) beta-carbonyl sulfides and (c,d,e) aryl sulfides with yield and enantiomeric excess (ee) percentages. The nature and size of the substrate influence the yield and ee values 
1.16 Dual mechanism proposed for CPO-catalyzed hydroxylations .....

1.17 Hydroxylation of 1,3-cyclohexadienes catalyzed by CPO. The nature and size of the substrate influence the yield and ee values

1.18 Oxidation of R-(+)-limonene catalyzed by CPO in the presence and absence of $\mathrm{KCl}$

1.19 A typical electronic absorption spectrum of a heme protein .

1.20 The Gouterman four orbital model

1.21 Interactions between iron $\mathrm{d} \pi(\mathrm{d}(\mathrm{xz}), \mathrm{d}(\mathrm{yz}))$ and porphyrin $\pi^{*}\left(\mathrm{a}_{1 \mathrm{u}}\right)$ orbitals

2.1 pCPO3.I-AmdS plasmid containing A. niger glucoamylase promoter (PglaA), CPO gene from $C$. fumago, $A$. nidulans anthranilate synthetase terminator $(\operatorname{Ttp} C)$, and $A$. nidulans acetamidase gene $(A m d S)$ as a selection marker allowing growth on acetamide as the sole source of nitrogen

$2.2 \mathrm{C} 29 \mathrm{H}$ pCPO sequencing results.

2.3 pAB4-1 plasmid containing A. niger PyrG gene as a selection marker allowing fungal growth without uridine supplementation

2.4 DNA electrophoresis. Restriction digestion of pCPO and pAB4-1. Lanes 1-4: pCPO3.I-AmdS, lanes 5-8 pAB4-1, lanes 1 and 5 - undigested DNA, lane 2 - HindIII, lane 3 - AflIII, lane 4 - Hind III and AflIII, lane 6 - XbaI, lane 7 - BamHI, lane 8 - XbaI and BamHI.

2.5 C29H CPO sample loaded on a gel filtration column. .46

2.6 SDS-PAGE. WT and C29H CPO. Lanes 1 and 2 - C29H, lane 3 - WT CPO

2.7 A plot of styrene concentration versus absorbance at $262 \mathrm{~nm}$ (average of 3 trials). Error bars represent standard deviation.

2.8 600-MHz proton NMR spectra of cyanide complexes of HRP, WT and C29H $\mathrm{CPO}$ in deuterated phosphate buffer, $\mathrm{pH}$ 5.9.

2.9 CD spectra of $1.0 \mu \mathrm{M}$ WT and C29H CPO in acetate buffer, pH 5.5. ...................55

2.10 UV-Vis spectra of WT and C29H CPO in 25 mM phosphate buffer, pH 5.9. .......56

$2.11 \mathrm{UV}$-Vis absorption spectra of WT CPO in [hos[hate or phosphate-citrate buffer at different $\mathrm{pH}$. 
2.12 UV-Vis absorption spectra of C29H CPO in phosphate or phosphate-citrate buffer in the acidic $\mathrm{pH}$ range..

2.13 UV-Vis absorption spectra of C29H CPO in phosphate-citrate buffer in basic pH 59

2.14 UV-Vis absorption spectra of ferric C29H, ferrous C29H, and ferrous C29H-CO complex in phosphate buffer, pH 5.9..................................................................60

2.15 UV-Vis absorption spectra of ferrous WT-CO and C29H-CO at pH 5.9................61

2.16 Locations of 3 cysteine residues in CPO. The following pdb file was used to

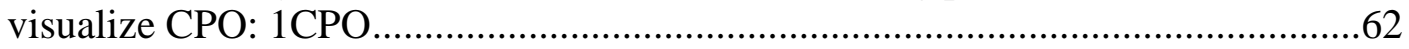

2.17 UV-Vis absorption spectra of ferrous $\mathrm{C} 29 \mathrm{H} / \mathrm{C} 79 \mathrm{H}-\mathrm{CO}$ and $\mathrm{C} 29 \mathrm{H} / \mathrm{C} 79 \mathrm{H} / \mathrm{C} 87 \mathrm{H}-\mathrm{CO}$ at $\mathrm{pH} 5.9$

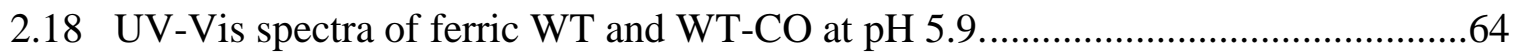

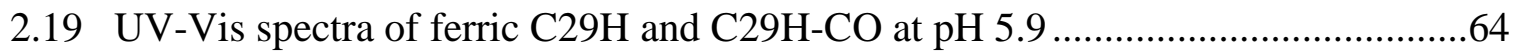

2.20 Chlorination of MCD to DCD by WT and C29H CPO at pH 2.75 ..........................66

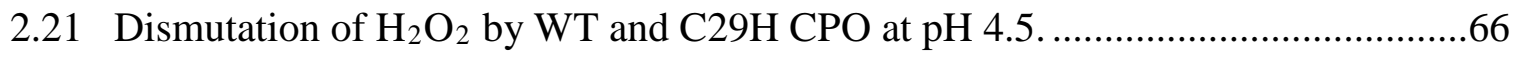

2.22 Epoxidation of styrene by WT and C29H CPO at pH 4.5....................................67

2.23 ABTS peroxidation by $\mathrm{WT}$ and $\mathrm{C} 29 \mathrm{H} \mathrm{CPO}$ at $\mathrm{pH} 4.5 \ldots \ldots \ldots \ldots \ldots \ldots \ldots \ldots \ldots \ldots \ldots \ldots \ldots \ldots \ldots . . .67$

2.24 Relative activities of WT (RZ=1.3-1.5) and C29H CPO (RZ=1.2-1.5) in chlorination ( $\mathrm{pH}$ 2.75), epoxidation ( $\mathrm{pH} 4.5)$, dismutation $(\mathrm{pH} 4.5)$, and peroxidation ( $\mathrm{pH} 4.5)$ assays

$2.25 \mathrm{pH}$ profile of chlorination activities of WT and C29H CPO..................................70

$2.26 \mathrm{pH}$ profile of dismutation activities of WT and C29H CPO...................................70

$2.27 \mathrm{pH}$ profile of epoxidation activities of WT and C29H CPO....................................71

$2.28 \mathrm{pH}$ profile of peroxidation activities of WT and C29H CPO.................................72

3.1 Hydrogen bond network in the proximal helix of CPO............................................75

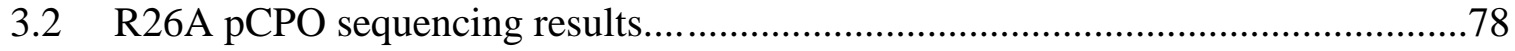




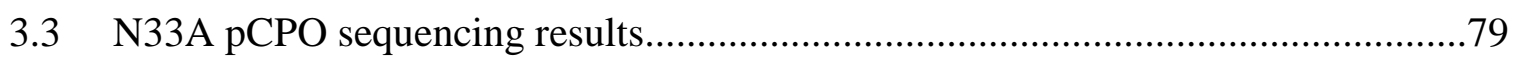

$3.4 \quad$ R26A/N33A pCPO sequencing results................................................................

3.5 R26A CPO sample loaded on a gel filtration column...............................................

3.6 SDS-PAGE. WT and R26A CPO. Lanes 1 and 2 - R26A, lane 3 - WT CPO.......80

3.7 SDS-PAGE. N33A CPO. WT - wild type CPO, 2-6 - N33A fractions from

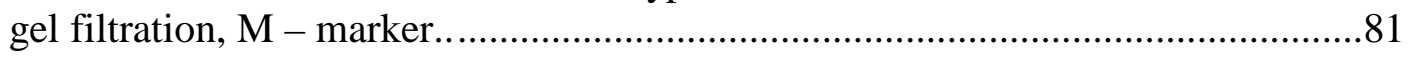

3.8 Secondary CD of $1.0 \mu \mathrm{M} \mathrm{WT}$ and mutant CPO in phosphate buffer, $\mathrm{pH} 5.9 \ldots \ldots . . .83$

3.9 Tertiary CD spectra of $16 \mu \mathrm{M} \mathrm{WT}$ and R26A CPO at pH 5.9..............................85

3.10 Tertiary CD spectra of $35 \mu \mathrm{M} \mathrm{WT}$ and N33A CPO at pH 5.9..............................85

3.11 Tertiary CD spectra of $11 \mu \mathrm{M}$ WT and R26A-N33A CPO in phosphate buffer,

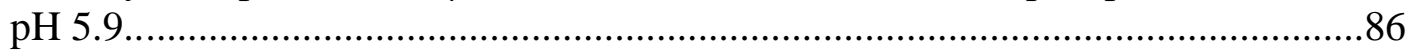

3.12 UV-Vis spectra of WT, R26A, N33A, and R26A/N33A CPO in 25 mM

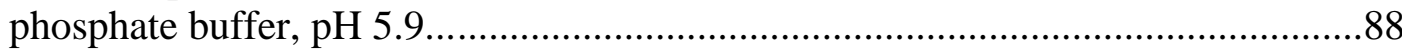

3.13 UV-Vis absorption spectra of N33A CPO in the acidic pH range..........................90

3.14 UV-Vis absorption spectra of N33A CPO in basic pH..........................................90

3.15 UV-Vis absorption spectra of R26A/N33A CPO in the acidic $\mathrm{pH}$ range.................91

3.16 UV-Vis absorption spectra of R26A/N33A CPO in basic pH.................................91

3.17 UV-Vis absorption spectra of R26A CPO at various pH......................................92

3.18 UV-Vis absorption spectra of ferric R26A, ferrous R26A, and ferrous R26A-CO

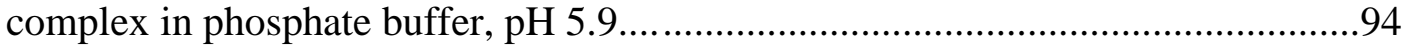

3.19 UV-Vis absorption spectra of ferric N33A, ferrous N33A, and ferrous N33A-CO complex in phosphate buffer, $\mathrm{pH}$ 5.9..

3.20 UV-Vis absorption spectra of ferric R26A/N33A, ferrous R26A/N33A, and Ferrous R26A/N33A-CO complex in phosphate buffer, pH 5.9..

3.21 Comparison of UV-Vis absorption spectra of ferrous mutant-CO complexes in phosphate buffer, pH 5.9. 
3.22 Cyanide (100 mM) binding to WT CPO in phosphate buffer, $\mathrm{pH}$ 6.0...................97

3.23 Cyanide $(100 \mathrm{mM})$ binding to R26A CPO in phosphate buffer, $\mathrm{pH}$ 6.0..............97

3.24 Cyanide (100 mM) binding to N33A CPO in phosphate buffer, $\mathrm{pH}$ 6.0...............98

3.25 Cyanide (100 mM) binding to R26A/N33A CPO in phosphate buffer, $\mathrm{pH}$ 6.0..... .98

3.26 Titration of WT CPO with cyanide in phosphate buffer, $\mathrm{pH}$ 6.0.

A. 2-10 $\mathrm{mM} \mathrm{CN}^{-}$. B. $10-800 \mu \mathrm{M} \mathrm{CN}^{-}$.

3.27 Titration of R26A CPO with $\mathrm{CN}^{-}(1-10 \mathrm{mM})$ in phosphate buffer, $\mathrm{pH}$ 6.0..........102

3.28 Titration of N33A CPO with $\mathrm{CN}^{-}(1-10 \mathrm{mM})$ in phosphate buffer, $\mathrm{pH}$ 6.0.........102

3.29 Titration of R26A/N33A with $\mathrm{CN}^{-}(1-10 \mathrm{mM})$ in phosphate buffer, $\mathrm{pH}$ 6.0.........103

3.30 $\mathrm{CN}^{-} \mathrm{Kd}$ determination for the mutant CPO (Only 1 trial for R26A was performed because of the limited amount of the protein, hence there are no error bars).

3.31 The Hill plot (determination of mutant CPO-CN binding stoichiometry).

The slopes of R26A, N33A, and R26A-N33A plots are 1.0048, 0.9943, and 1.0249, respectively.

3.32 Cyanide (100 mM) binding to WT CPO in phosphate buffer, $\mathrm{pH} 12$...................105

3.33 Cyanide (100 mM) binding to N33A in phosphate buffer, $\mathrm{pH} 12.0 \ldots \ldots \ldots \ldots \ldots \ldots \ldots . . . . .105$

3.34 Cyanide (100 mM) binding to R26A/N33A in phosphate buffer, $\mathrm{pH} 12.0 \ldots \ldots \ldots . .106$

4.1 $\mathrm{pH}$ profiles of WT and mutant CPO chlorination activities... ...........................115

4.2 pH profiles of R26A, N33A, and R26A/N33A CPO chlorination activities.........115

4.3 pH profiles of WT, R26A, N33A, and R26A/N33A CPO dismutation activities .116

$4.4 \mathrm{pH}$ profiles of WT and mutant CPO peroxidation activities...............................118

$4.5 \mathrm{pH}$ profiles of WT and R26A CPO epoxidation activity.................................119

4.6 Relative peroxidation activities of WT and mutant CPOs at $\mathrm{pH} 2.75 \ldots \ldots \ldots \ldots \ldots . . . . .121$

4.7 Relative peroxidation activities of WT and mutant CPOs at $\mathrm{pH} 4.5 \ldots \ldots \ldots \ldots \ldots \ldots . . .121$ 
4.8 Relative epoxidation activities of WT and mutant CPOs at $\mathrm{pH} 4.5 \ldots \ldots \ldots \ldots \ldots \ldots . . . . .122$

4.9 Relative dismutation activities of WT and mutant CPOs at $\mathrm{pH} 4 \ldots \ldots \ldots \ldots \ldots \ldots \ldots . . . . . . . . . .122$

4.10 Relative chlorination activities of WT and mutant CPOs at $\mathrm{pH} 2.75 \ldots . . . . . . . . . . . . .123$

A.1 Primers used for construction of C29H/C79H and C29H/C79H/C87H CPO (His mutation is shown in bold)............................................................149

A.2 Nucleotide sequence of full-length WT CPO gene.........................................149

A.3 Amino acid sequence of WT CPO including signal peptide (1-20) and propeptide (322-373).. .150 


\section{LIST OF ABBREVIATIONS}

ABBREVIATION

AaeUPO

A. aegerita

ABTS

Ala/A

Amds

A. nidulans

A. niger

APO

Arg/R

A. rusticana

Asn/N

Asp/D

B. bubalis

$\mathrm{Cc}_{C} \mathrm{P}$

CD

\section{FULL NAME}

Unspecific peroxygenase from Agrocybe aegerita

Agrocybe aegerita

2,20 -azino-bis(3-ethylbenzothiazoline-6- sulfonic acid)

Alanine

Acetamidase gene

Aspergillus nidulans

Aspergillus niger

Ascorbate peroxidase

Arginine

Armoracia rusticana

Asparagine

Aspartic acid

Bubalus bubalis

Cytochrome $c$ peroxidase

circular dichroism 


\begin{tabular}{|c|c|}
\hline CDE & Chlorite-dismutase-DyP-EfeB \\
\hline C. fumago & Calderiomyces fumago \\
\hline Cld & Chlorite dismutase \\
\hline Cpd 0 & Compound 0 \\
\hline Cpd I & Compound I \\
\hline Cpd II & Compound II \\
\hline Cpd X & Compound X \\
\hline $\mathrm{CPO}$ & Chloroperoxidase \\
\hline CT & Charge transfer \\
\hline CYP & Cytochrome P450 monooxygenase \\
\hline Cys/C & Cysteine \\
\hline DCD & Dichlorodimedone \\
\hline DEAE & Diethylaminoethanol \\
\hline DNA & Deoxyribonucleic acid \\
\hline dNTP & Deoxyribonucleotide triphosphate \\
\hline DyP & Dye-decolorizing peroxidase \\
\hline еe & Enantiomeric excess \\
\hline
\end{tabular}




\begin{tabular}{|c|c|}
\hline EPO & Eosinophil peroxidase \\
\hline $\mathrm{Gln} / \mathrm{Q}$ & Glutamine \\
\hline Glu/E & Glutamic acid \\
\hline Gly/G & Glycine \\
\hline $\mathrm{HF}$ & High fidelity \\
\hline $\mathrm{His} / \mathrm{H}$ & Histidine \\
\hline HOMO & Highest occupied molecular orbital \\
\hline HRP & Horseradish peroxidase \\
\hline KatG & Catalase-peroxidase \\
\hline Leu/L & Leucine \\
\hline LiP & Lignin peroxidase \\
\hline LPO & Lactoperoxidase \\
\hline LUMO & Lowest unoccupied molecular orbital \\
\hline $\mathrm{Mb}$ & Myoglobin \\
\hline MCD & Monochlorodimedone \\
\hline MM & Minimal medium \\
\hline $\mathrm{MnP}$ & Manganese peroxidase \\
\hline
\end{tabular}


MPO

$\mathrm{NAD}(\mathrm{P}) \mathrm{H}$

NMR

NOS

P. chrysosporium

PCR

PDB

PglaA

Phe/F

Pro/P

$\mathrm{RR}$

RZ value

SA

S. cerevisiae

SDS-PAGE

Ser/S

T. cucumeris
Myeloperoxidase

Nicotinamide adenine dinucleotide (phosphate)

Nuclear magnetic resonance

Nitric oxide synthase

Phanerochaete chrysosporium

Polymerase chain reaction

Protein data base

glucoamylase promoter

Phenylalanine

Proline

Resonance Raman

Reinheitzahl value

Specific activity

Saccharomyces cerevisiae

Sodium dodecyl sulfate polyacrylamide gel electrophoresis

Serine

Thanatephorus cucumeris 
$\mathrm{Thr} / \mathrm{T}$

TPO

Trp/W

TtrpC

Tyr/T

UPO

$\mathrm{Val} / \mathrm{V}$

VPO

WT
Threonine

Thyroid peroxidase

Tryptophan

anthranilate synthetase terminator

Tyrosine

Unspecific peroxygenase

Valine

Versatile peroxidase

Wild-type 


\section{CHAPTER I.}

\section{HEME PROTEIN DIVERSITY: STRUCTURE, FUNCTION, APPLICATIONS}

\subsection{Introduction to heme proteins}

Heme proteins are ubiquitous in both eukaryotic and prokaryotic organisms. They carry out a variety of biological functions that includes oxygen transport, electron transfer, signal transduction (via sensing diatomic gases), and catalysis of various redox reactions (Table 1.1).

Table 1.1. Heme proteins and their biological functions.

\begin{tabular}{lll}
\hline Function & Proteins & References \\
\hline $\mathrm{O}_{2}$ transport or & Myoglobin $(\mathrm{Mb})$ & $(1-3)$ \\
storage & Hemoglobin & $(1-3)$ \\
& Leghemoglobin & $(4)$ \\
& Neuroglobin & $(5-6)$ \\
& Cytoglobin & $(7)$ \\
\hline Electron transfer & Cytochromes $a, b$, and $c$ & $(8-9)$ \\
\hline Signal transduction & Guanylate cyclase (NO, CO) & $(10-12)$ \\
& FixL (O 2$)$ & $(13-15)$ \\
& EcDos $\left(\mathrm{O}_{2}, \mathrm{NO}, \mathrm{CO}\right)$ & $(16-17)$ \\
& NPAS2 $(\mathrm{CO})$ & $(18-19)$ \\
& HemAT $\left(\mathrm{O}_{2}\right)$ & $(20)$ \\
& CooA $(\mathrm{CO})$ & $(21-22)$ \\
\hline Catalysis & Peroxidases & $(23-28)$ \\
& Catalases & $(32-33)$ \\
& NO synthase & $(30)$ \\
& Cytochromes P450 & \\
\hline
\end{tabular}


Each class of heme proteins contains one or two heme moieties as the prosthetic group. In general, the heme consists of a ferrous iron centered inside a substituted heterocyclic porphine molecule known as porphyrin. The nature and location of the substituents on a porphine determines the type of heme $\left(a, b, c, d_{1}, f, o\right)$. The most frequently encountered heme groups are shown in Fig. 1.1. The majority of heme proteins possess heme $b$, also known as iron protoporphyrin IX (34). Enzymes containing non-b heme groups include cytochromes $a$, cytochromes $c$, nitrite reductases, cytochromes $b 6 f$, and heme-copper oxidases that possess heme $a, c, d_{1}, f$, and $o$, respectively (35-39).

Since iron protoporphyrin IX is the most common type of heme found in heme proteins, the following discussion will concentrate on $b$-type heme proteins. The iron in the heme group is coordinated to the four nitrogens of the porphyrin in addition to one or two axial ligands that usually include such amino acids as histidine, cysteine, tyrosine, methionine, or lysine (40). In many heme proteins, the heme group is located in proximity to an alpha-helix parallel to the porphyrin plane that is responsible for heme-protein packing interactions (41). The extent of heme exposure to solvent plays a role in determining biochemical properties of the protein (42). In addition, the type of the heme group together with the nature of metal ligation and the proximal and distal environment in the active site determine the reactivity of heme enzymes and their selectivity toward substrates (43-45).

The following two sections will describe heme proteins that carry out various catalytic functions and utilize either hydrogen peroxide or molecular oxygen to perform a plethora of oxidation reactions. I will concentrate my discussion on the family of enzymes called peroxidases and, particularly, on fungal chloroperoxidase (CPO) since it is the subject of 
my research. Structural, spectroscopic, and catalytic properties of CPO will be discussed in more detail.

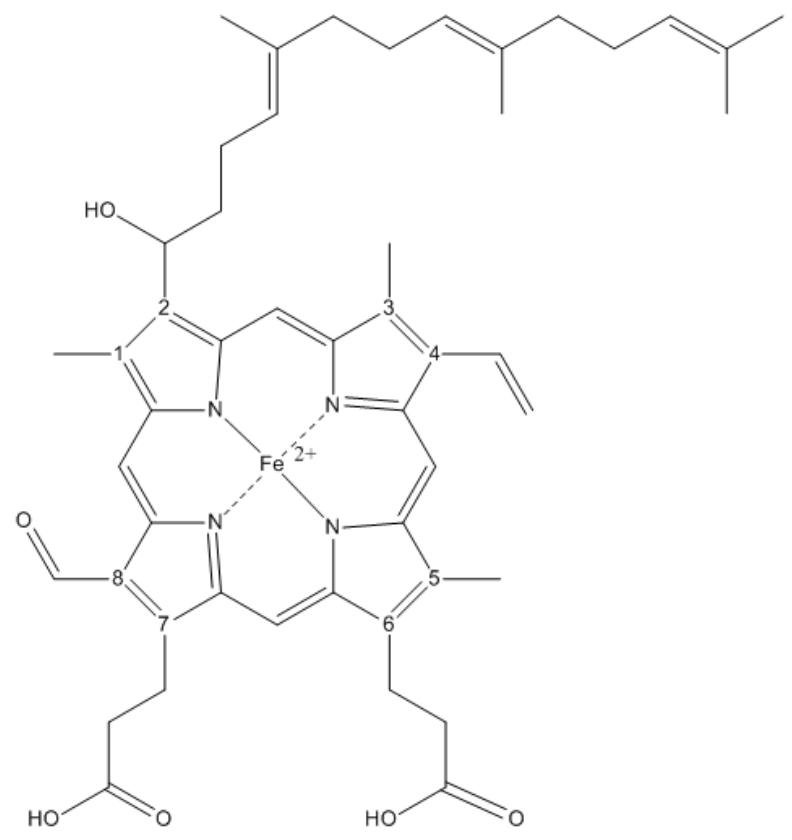

Heme $a$

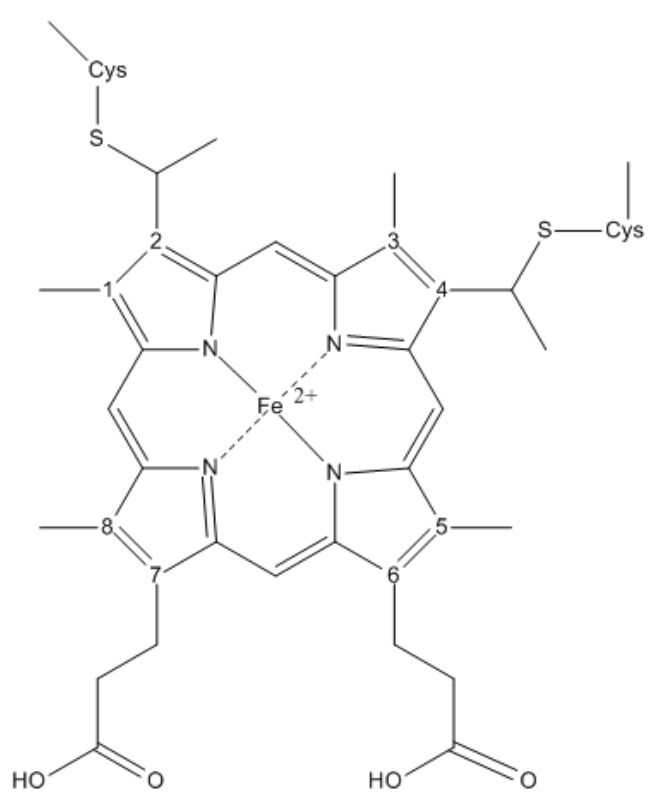

Heme $c$

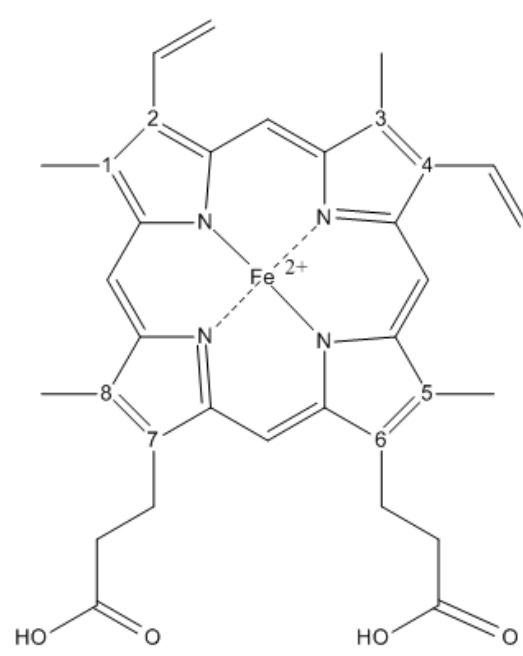

Heme $b$

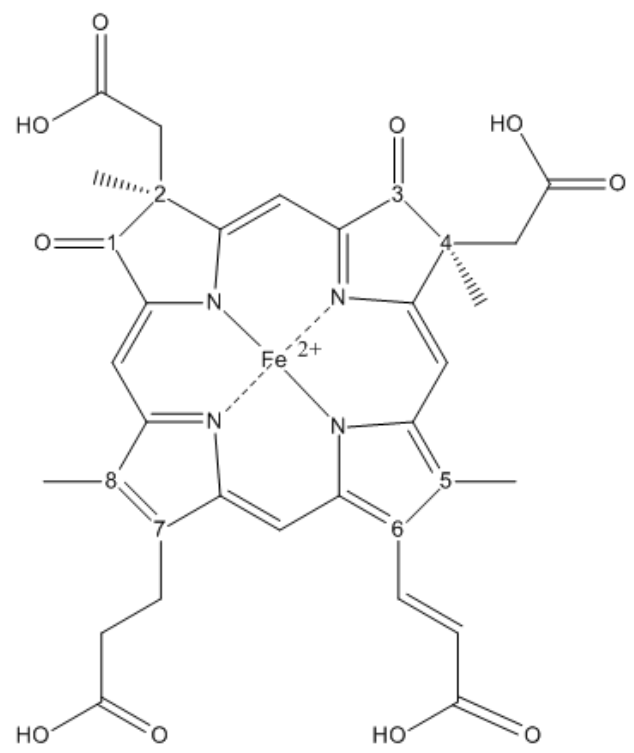

Heme $d_{1}$

Figure 1.1. Structures of heme groups most frequently found in heme proteins. 


\subsection{Peroxidases}

Classical peroxidases are enzymes capable of catalyzing one-electron oxidations of a variety of substrates, using hydrogen peroxide as an oxidizing agent. The majority of peroxidases contain a heme group as the co-factor; however, a few are known to utilize other co-factors. For example, glutathione peroxidase and phospholipid hydroperoxide glutathione peroxidase utilize selenium as a co-factor (46). Originally, peroxidases were grouped into two major families: animal and plant peroxidases. However, over time peroxidases were found in other organisms including bacteria, and many peroxidases were found across different kingdoms of life. Moreover, some peroxidases were found to be able to catalyze reactions that are usually performed by other biological catalysts such as catalases, oxygenases, and peroxygenases. Therefore, a more appropriate classification of peroxidases reflects the types of reactions catalyzed by a particular family. According to this classification, heme peroxidases are grouped into 4 superfamilies: peroxidasescatalases, peroxidases-cyclooxygenases, peroxidases-peroxygenases, and CDE (chloritedismutase-DyP-EfeB) heme peroxidases (47-49). The members of each protein superfamily are given in Table 1.2.

\subsubsection{Peroxidase-catalases}

Peroxidase-catalases, in addition to catalyzing classical peroxidation reactions, are able to perform the decomposition of hydrogen peroxide, a reaction typically catalyzed by regular catalases to protect cells from oxidative damage (30). Peroxidase-catalases are the most diverse and abundant superfamily, and is further divided into subfamilies of Class I, Class II, and Class III whose representatives belong to intracellular, fungal extracellular, and plant extracellular proteins, respectively. All three classes of peroxidases-catalases 
contain conserved, catalytically important distal Arg-Trp/Phe-His and proximal HisTrp/Phe-Asp triads (Fig. 1.2 A-C) (63-66). Proximal heme ligation to a His is similar to ligation in other peroxidase families (except peroxygenases) and in contrast to a tyrosine proximal heme ligand in monofunctional catalases (67). The proximal His is coordinated to the iron of the heme through $\mathrm{N}^{\varepsilon}$, and the distal His acts as the acid-base catalyst during the formation of Compound I, which is discussed below.

Table 1.2. Classification of heme peroxidases.

\begin{tabular}{lll}
\hline Superfamily & Representative proteins & References \\
\hline Peroxidase-catalases & & $(50)$ \\
Class I (intracellular) & Catalase-peroxidases (KatGs) & $(51)$ \\
& Cytochrome c peroxidase (CcP) & $(52)$ \\
& Ascorbate peroxidase (APO) & $(24)$ \\
Class II (extracellular fungal) & Lignin peroxidase (LiP) & $(25)$ \\
Class III (extracellular plant) & Versatile peroxidase (VPO) & $(53)$ \\
(Welinder 1992) & Horseradish peroxidase (HRP) & $(23)$ \\
\hline Peroxidase-cyclooxygenases & Myeloperoxidase (MPO) & $(26)$ \\
& Eosinophil peroxidase (EPO) & $(54)$ \\
Clds, EfeB, & Lactoperoxidase (LPO) & $(55)$ \\
DyPs A, B, C, and D & Thyroid peroxidase (TPO) & $(56)$ \\
\hline Peroxidase-peroxygenases & Chloroperoxidase (CPO) & $(52-33)$ \\
& Unspecific peroxygenases (UPOs) \\
\hline Chlorite dismutases (Clds) & $(59-60)$ \\
\hline & Dye-decolorizing peroxidases (DyPs) & $(61)$ \\
\hline & EfeB & \\
\hline
\end{tabular}




\subsubsection{Peroxidase-cyclooxygenases}

The members of the peroxidase-cyclooxygenase superfamily catalyze the oxygenation of fatty acids in the formation of prostaglandins which are autocrine and paracrine lipid mediators involved in pain sensitivity, fever regulation, cell growth control, and hormonal regulation (68). The superfamily includes mammalian, plant, fungal, and bacterial proteins. Mammalian cyclooxygenases are the most studied and include myeloperoxidase, eosinophil peroxidase, lactoperoxidase, and thyroid peroxidase. Active sites of mammalian cyclooxygenases possess two ester linkages between the heme group and conserved distal aspartate and glutamate residues. Other conserved residues include distal His and Asp together with proximal His hydrogen bonded to Asn (Fig. 1.2 D, 69). Distal His is involved in the formation of catalytic species, Compound I, while distal glutamine residue and several water molecules form a hydrogen-bond network involved in halide delivery and binding (47).

\subsubsection{CDE peroxidases}

The CDE superfamily consists of dye decolorizing peroxidases, chlorite dismutases, and EfeB enzymes that are found in fungal, archeal, and bacterial phyla. This superfamily of heme enzymes has only been recently identified and only a few members have crystal structures available that have been biochemically characterized. The active sites of known CDE proteins possess a His-Asp/Glu pair in the proximal pocket and an Asp-Arg pair in the distal pocket (49).

In contrast to a distal His in other peroxidases, CDE enzymes use aspartic acid in their catalysis (70-72). The reactions that are catalyzed by this superfamily include but are not limited to: oxidations of azo dyes such as Reactive Black 5, 2,6-dimethoxyphenol, and 
other substituted phenols, 2,20 -azino-bis(3-ethylbenzothiazoline-6-sulfonic acid (ABTS), $\beta$-carotene, ascorbic acid, and anthraquinone dyes such as Reactive Blue 19, Reactive Blue $5(73-79)$.
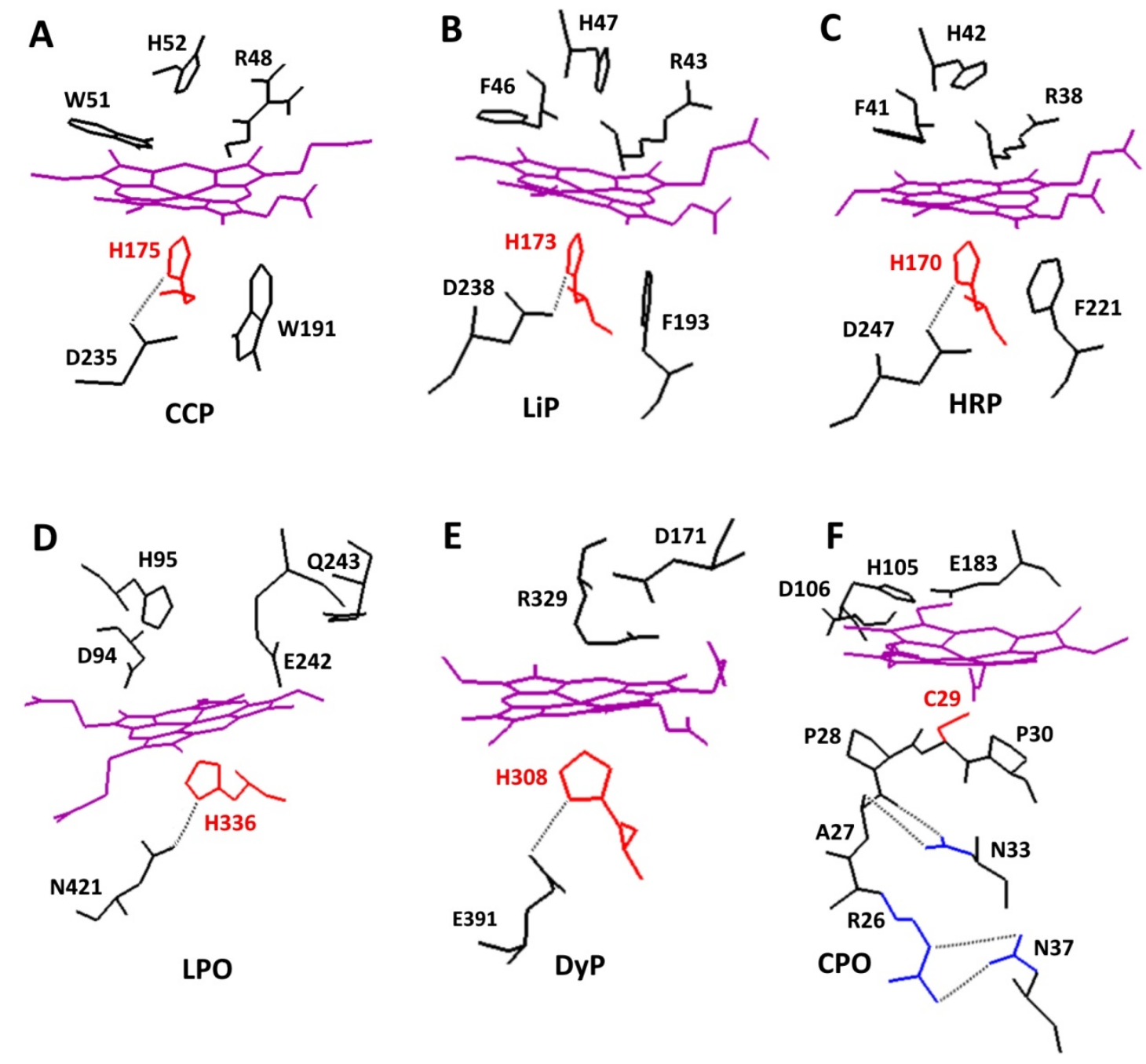

Figure 1.2. Active site structures of cytochrome c peroxidase from Saccharomyces cerevisiae (A), lignin peroxidase from Phanerochaete chrysosporium (B), horseradish peroxidase from Armoracia rusticana (C), lactoperoxidase from Bubalis bubalis (D), dye decolorizing peroxidase from Thanatephorus cucumeris (E), and chloroperoxidase from Calderiomyces fumago (F). The following pdb files were used to visualize the active sites: 1CCA, 1B82, 1ATJ, 2GJM, 3AFV, 1CPO (64-66, 69-71). 


\subsubsection{Peroxidase-peroxygenases}

Peroxidase-peroxygenases, besides catalyzing peroxidation reaction, are able to incorporate oxygen from hydrogen peroxide into various substrates. The most studied member of this superfamily is chloroperoxidase from the Caldariomyces fumago fungus, which is a focus of my research and will be discussed in detail in section 1.3. Other enzymes belonging to this group have only recently been discovered and characterized. Examples include unspecific peroxygenases (UPOs) from the basidiomycetous fungi Agrocybe aegerita (AaeUPO), Coprinus radians, and Marasmius rotula (80-82). These UPOs are functional peroxygenases that transfer an oxygen atom from hydrogen peroxide to diverse organic substrates (aromatics, heterocycles, linear and cyclic alkanes/ alkenes, fatty acids, etc. (83-86). In addition to these reactions, UPOs are able to catalyze O- and N-dealkylation, dechlorination, and halide oxidation (87).

A feature that distinguishes this superfamily from other heme peroxidases is the use of a Cys residue as the proximal heme ligand instead of a His. Furthermore, active sites of known peroxygenases and CPO share common structural motifs R(G/A)PCP(G/A)LN in the proximal side and $\mathrm{E}(\mathrm{N} / \mathrm{H}) \mathrm{D}$ in the distal side (Fig. 1.3). In addition, the proximal alphahelix in both CPO and AaeUPO has a similar orientation, being almost perpendicular to the heme plane. The orientation of this alpha-helix has been suggested to fine-tune the redox potential of the heme (88).

It has been proposed that UPOs are structural hybrids between classical peroxidases and cytochrome P450 monooxygenases (CYPs), combining the more polar distal side of peroxidases with the proximal thiolate ligation of CYPs (Fig. 1.3) (62). CYPs are hemethiolate proteins, whose main function is oxygen insertion into organic substrates using 
molecular oxygen and $\mathrm{NAD}(\mathrm{P}) \mathrm{H}(91)$. However, some CYPs, such as $\mathrm{P} 450{ }_{\mathrm{SP} \alpha}$ from Sphingomonas paucimobilis and $\mathrm{P} 450_{\mathrm{BS} \beta}$ from Bacillius subtilis, are capable of using hydrogen peroxide or organic peroxide through the 'peroxide-shunt' pathway in the absence of $\mathrm{NAD}(\mathrm{P}) \mathrm{H}$ (92-93). Peroxygenation reactions carried out by P450s are, however, much less efficient than those performed by true peroxygenases. Since peroxygenases combine structural features of peroxidases and CYPs, they allow for the utilization of a catalytic cycle of classical heme peroxidases with the "peroxide shunt" of P450s in their catalysis. Interestingly, spectral studies on CPO and AaeUPO suggest that the latter is closer to cytochromes P450 than to CPO and may constitute a functional hybrid of the two (94).
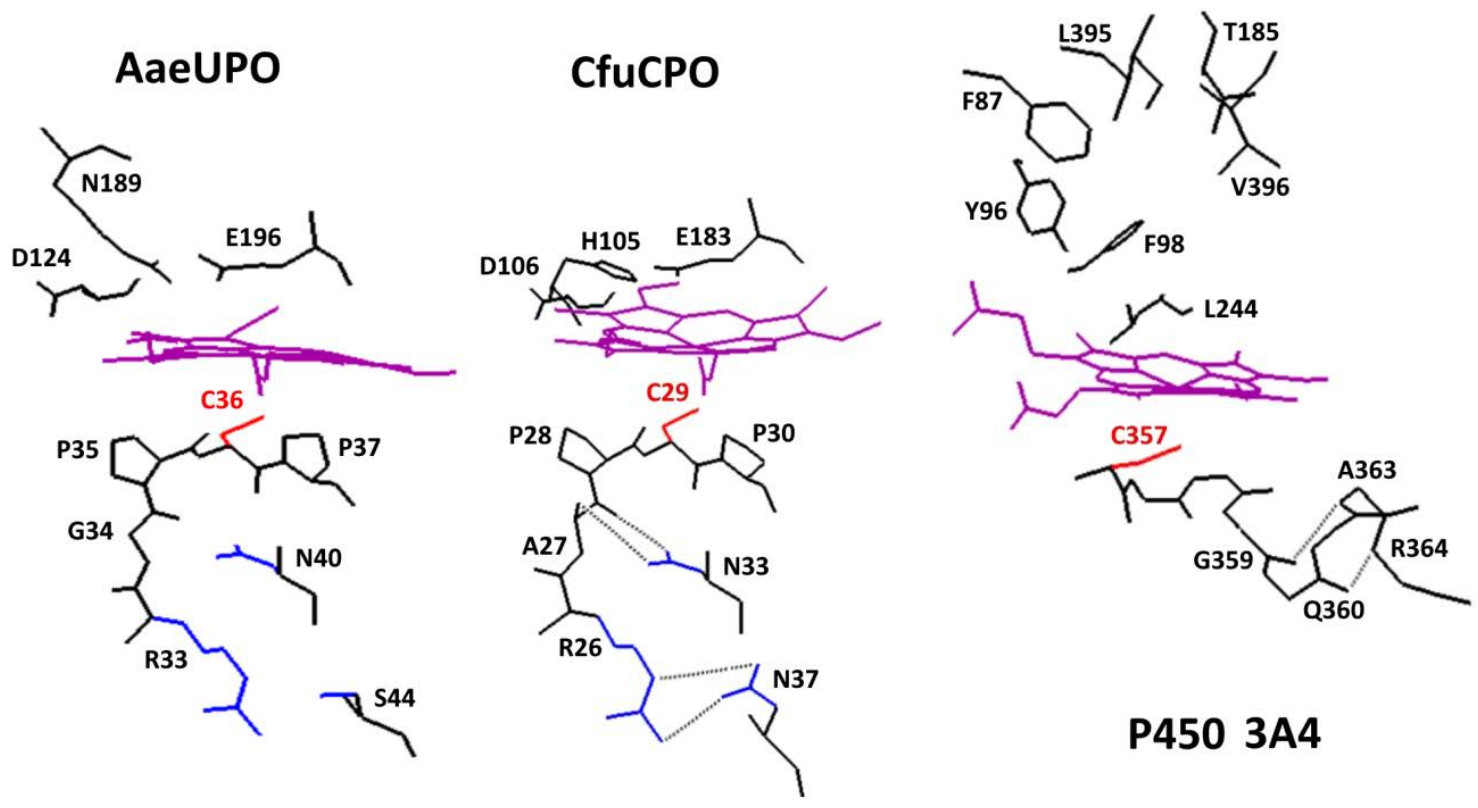

P450 3A4

Figure 1.3. Active site structures of aromatic peroxygenase from A. aegerita (A), chloroperoxidase from $C$. fumago (B), and cytochrome P450 3A4 from Homo sapiens (C). The following pdb files were used to visualize the active sites: $2 \mathrm{YOR}, 1 \mathrm{CPO}$, and 1W0E $(71,89,90)$. Heme is depicted in purple color and proximal axial ligands are indicated in red. Side chains of the proximal amino acids involved in hydrogen bond network (subject of chapters 3 and 4) formation are shown in blue. 


\subsection{Chloroperoxidase}

Chloroperoxidase, produced by the ascomycetous fungus Caldariomyces fumago, is a heme-containing protein that belongs to the peroxidase-peroxygenase family. The biological function of this extracellular protein is proposed to be the chlorination of 2chlorocyclopentane-1,3-dione to 2,2-dichlorocyclopentane-1,3-dione, which is a precursor of caldariomycin, a compound with antibacterial properties, thus providing the fungus with protection against microbes (95). In addition to halogenation, CPO is able to carry out catalysis of a plethora of oxidative reactions that are characteristic of other enzyme classes, using hydrogen peroxide as an oxidizing agent. The catalytic diversity of CPO has been attributed to its unique structure and will be discussed below.

\subsubsection{The structure of CPO}

Chloroperoxidase is a heavily glycosylated heme-containing protein with a molecular weight of approximately 42 kilodaltons (95). It is initially synthesized as a 373-amino-acid precursor polypeptide and then underdoes extensive post-translational modifications that include removal of a 21-amino-acid signal peptide from the N-terminus, removal of a 53amino-acid sequence from the C-terminus that aids in correct folding, cyclization of $\mathrm{N}$ terminal glutamic acid into pyroglutamic acid, $\mathrm{N}$ - and O-glycosylations, deamidation of three amidic residues to their corresponding acids, a disulfide bond formation, and incorporation of heme b $(71,96,97)$. Interestingly, two isoforms of CPO were identified depending on the glycosylation pattern, with the major one possessing 21 sugar groups located at $3 \mathrm{~N}$ - and 11 O-glycosylation sites (71).

Similarly to other heme peroxidases, CPO is folded into $\mathrm{N}$ - and C-terminal domains with the heme group located between the domains. The secondary structure of CPO 
includes 8 alpha helices (A-H) and one small beta pair (Fig. 1.4). However, the overall fold is different from that of classical peroxidases or P450s. In CPO, the proximal heme ligand, Cys 29, is located in the N-terminal part of the protein in the N-terminus of the helix A (residues 29-38) that is almost perpendicular to the heme plane (71). In CYPs and other peroxidases, the proximal ligand is situated in the C-terminal domain of the protein in the N-terminus of the helix that is parallel to the heme (98). It has been proposed that electropositive environment of helix $\mathrm{A}$ at the $\mathrm{N}$-terminus fine-tunes the electronegativity of the proximal Cys and influences structural, catalytic, and ligand binding properties of СРО $(88,99,100)$.

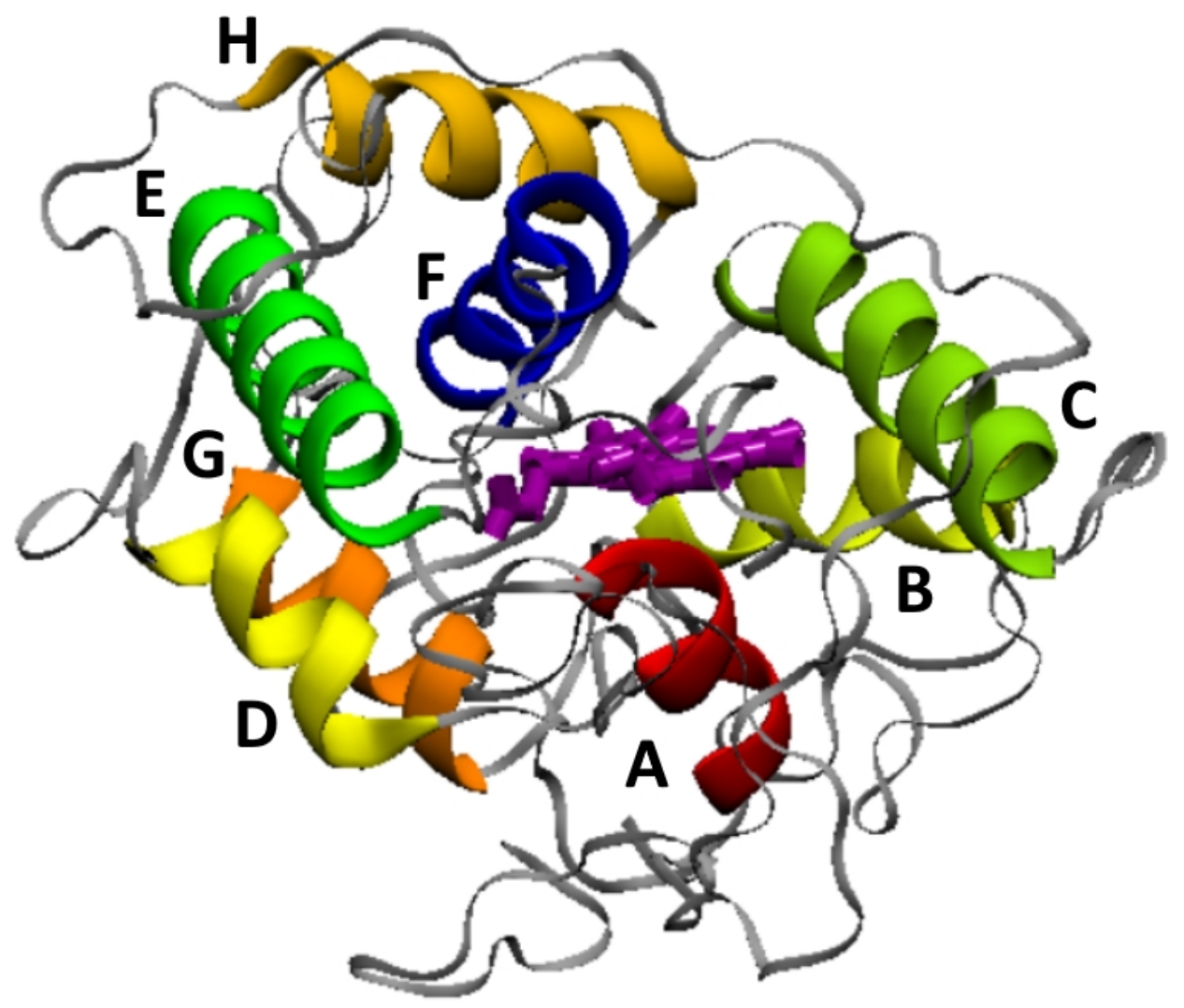

Figure 1.4. Crystal structure of chloroperoxidase from C. fumago. The following PDB file was used to visualize CPO: 1CPO (71). Heme is depicted in purple color. Letters indicate different helices in CPO structure. 
The proximal Cys in CPO is hydrogen-bonded (H-bonded) to the peptide NH groups of Ala 31 and Leu 32 situated at the N-terminus of the helix A. Hydrogen bonding between Cys and polypeptide amides is a common feature of other thiolate proteins including P450s, in which proximal Cys 357 is H-bonded to NH groups of Leu 358, Gly 359, and Gln 360 (98). These H-bonds contribute to the increase of the iron redox potential by decreasing the negative charge on the Cys ligand, and stabilize the thiolate by preventing its protonation and protecting against diatomic reactants, all of which result in the formation of inactive forms of the enzyme $(101,102)$.

The active site of CPO shares common structural features with both peroxidases and P450 enzymes (Figs. 1.2 and 1.3). In CPO, the heme iron is coordinated to the proximal Cys, similarly to P450s and in contrast to the proximal His of traditional peroxidases (71). However, the distal pocket of CPO, which is similar to that of classical peroxidases, is dramatically different from that of P450s which contains mainly non-polar residues. Some distal residues are involved in the formation of Compound I (Cpd I), an active species in the catalytic cycle of most heme enzymes. In classical peroxidases, the distal His is hydrogen-bonded to an Arg residue and is responsible for the peroxide bond cleavage in Cpd I formation. In CPO however, this function is carried out by Glu 183, which is hydrogen-bonded to the distal His 105. Arginine and histidine provide a particular orientation to the His and Glu, respectively. Therefore, the CPO active site environment is somewhat similar to other peroxidases in terms of polarity and spacial arrangement; however, it also resembles p450-type enzymes because of the thiolate proximal ligand as well as possessing direct accessibility to the heme through a wide channel (Fig. 1.5). Access to the heme is typically restricted in classical peroxidases (103). CPO's wide 
channel provides access to bulky substrates, such as those involved in oxygen insertion reactions, and is likely to be responsible for the observed enantioselectivity of CPO. In addition, there is a narrow channel connecting the heme center with the protein surface. Bulky and hydrophobic residues make up the narrow channel near the heme center, while small and polar residues are located near the exit to the protein surface. The narrow channel contains multiple halide binding sites and is proposed to provide halogen ion access to the $\mathrm{CPO}$ active site. It should be noted that in peroxidases the heme access by substrates is restricted; however, the heme edge is accessible by substrates for one-electron oxidations, which is in contrast to CPO whose heme edge is inaccessible to most substrates $(71,103)$. It is likely that the unique and unusual structure of CPO facilitates its diverse catalytic capabilities.

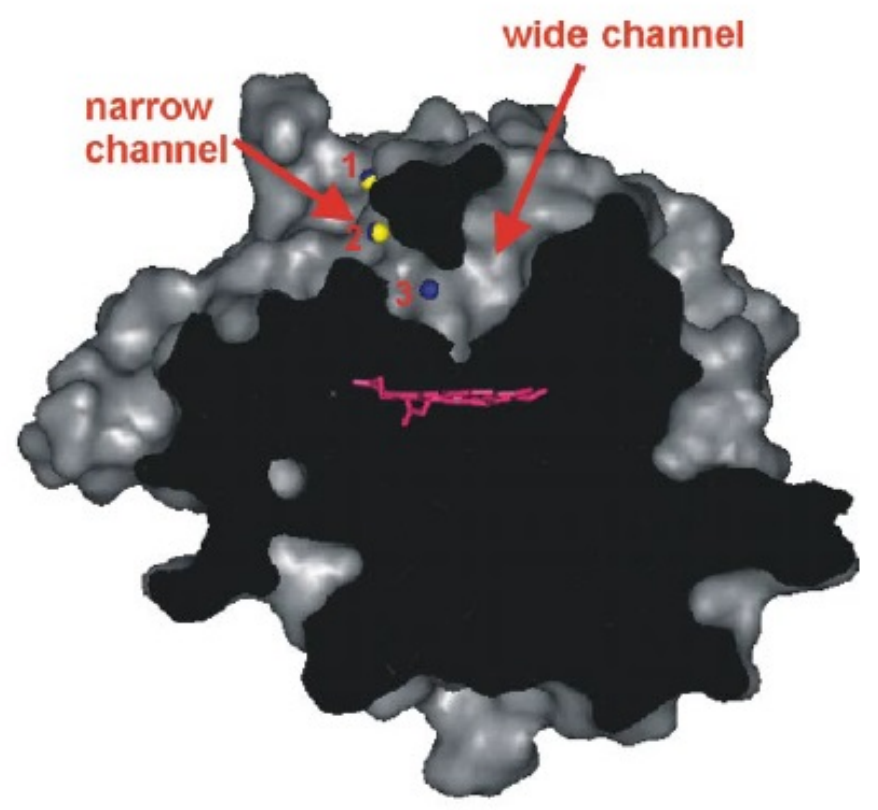

Figure 1.5. Slice through surface representation of CPO. The two channels are marked by arrows, and the heme is shown in magenta. The bromide and iodide binding sites are depicted by yellow and blue spheres, respectively (103). 


\subsubsection{Overview of CPO catalytic cycle}

As previously mentioned, $\mathrm{CPO}$ is unique because it is able to catalyze various reactions that are usually performed by different groups of enzymes. The catalytic pathways are summarized in the diagram created by L. P. Hager (Fig. 1.6).

The initial step of CPO catalytic cycle is the conversion of the native ferric enzyme (resting state) to the oxo-ferryl cation-radical intermediate $\left(\left[\mathrm{Fe}^{4+}=\mathrm{O}\right]^{\cdot+}\right)$ called Compound I. In many heme enzymes, Cpd I is efficient in catalyzing only one particular type of reaction, depending on the class of the enzyme. In the case of CPO, however, once Cpd I is formed, it is able to follow different catalytic pathways. For example, it can either form oxo-ferryl Compound II (Cpd II, $\mathrm{Fe}^{4+}=\mathrm{O}$, peroxidation pathway), oxo-ferric Compound X (Cpd X, $\mathrm{Fe}^{3+}=\mathrm{O}$, halogenation pathway), or return to its resting state $\left(\mathrm{Fe}^{3+}\right.$, dismutation and P450 pathways). Such catalytic diversity distinguishes CPO from other biocatalysts and attracts the attention of scientists from various fields.

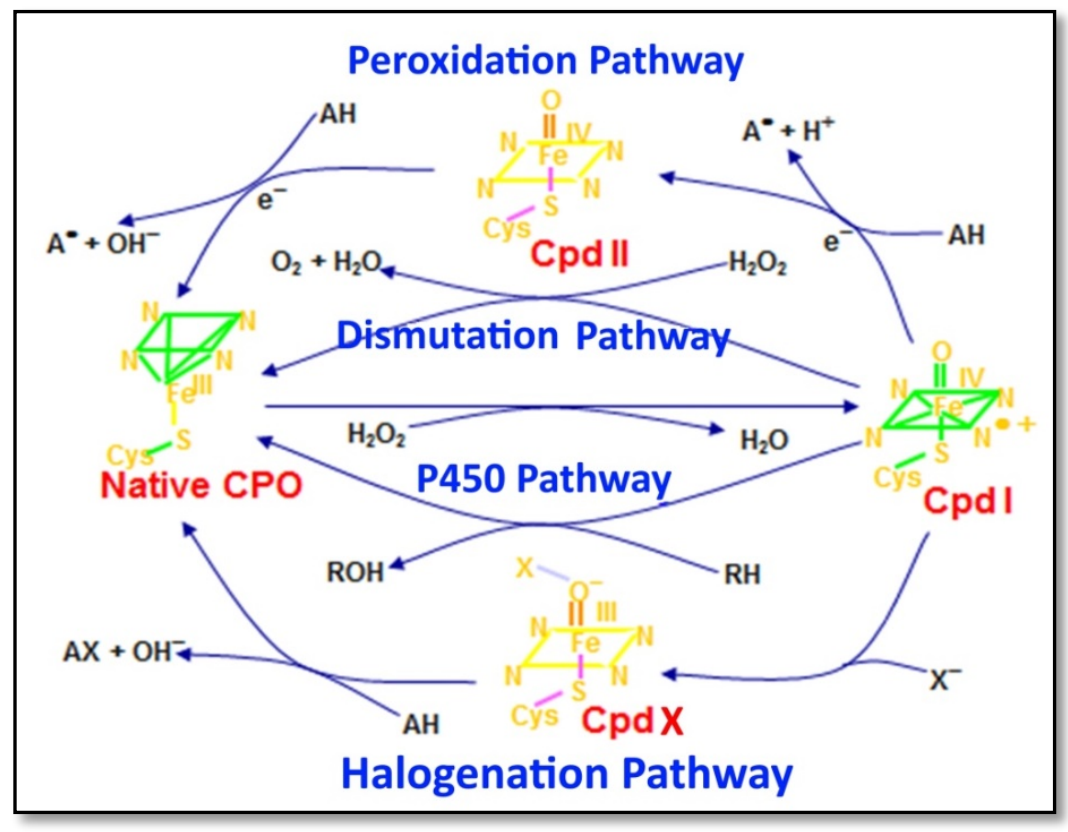

Figure 1.6. Catalytic cycle of CPO (created by L. P Hager). 


\subsubsection{Active intermediates: Compound I, Compound II, and Compound X.}

Compound I, an oxo-ferryl cation-radical, is an active intermediate in the catalytic cycle of all reactions catalyzed by CPO and is similar to that in other heme enzymes (104). The mechanism of Cpd I formation begins with the formation of Compound 0, as proposed by Chen et al. (Fig. 1.7) (105). An oxygen atom of hydrogen peroxide binds to the heme iron to form $\mathrm{Fe}(\mathrm{III}) \mathrm{HOOH}$ in two possible conformations, depending on whether Glu183 hydrogen bonds to the hydrogen of the proximal or distal oxygen. However, Shaik et al. have shown that interactions with the distal hydrogen will not lead to the production of Cpd I, which leaves the first possibility. In this conformation, Glu 183 abstracts a proximal proton concomitantly with the movement of the $-\mathrm{OOH}$ group about the $\mathrm{z}$-axis of the heme plane, resulting in formation of Cpd 0, in which protonated Glu 183 is hydrogen bonded to the distal oxygen. In the next step, Glu 183 protonates distal oxygen and upon water release, Cpd I is formed. Interestingly, the rate of Cpd I formation was shown to be only slightly dependent on the nature of the proximal ligand and independent of $\mathrm{pH}$. However, the axial ligand might influence the stability and reactivity of Cpd I as was shown in cytochrome $c$ peroxidase (106).

Another intermediate, Cpd II, is an oxo-ferryl species in the peroxidation pathway of CPO formed from Cpd I. The X-ray absorption fine structure study by Green et al. has demonstrated that there are three species of Cpd II (107). It was postulated that the major species of Cpd II is in the protonated form, while the minor species is in the de-protonated form. The third species had a low yield (6\%) and could not be assigned. A computational study by Lai et al. further explored the nature of Cpd II and assigned both the major and minor species to be iron(IV)-hydroxide species, differing only in hydrogen bonding with 
either a water molecule or Glu 183 (108). The third species was tentatively assigned to be an iron(III)-hydroxide cation radical; however, no experimental data have confirmed the nature of Cpd II proposed by Green and Lai.

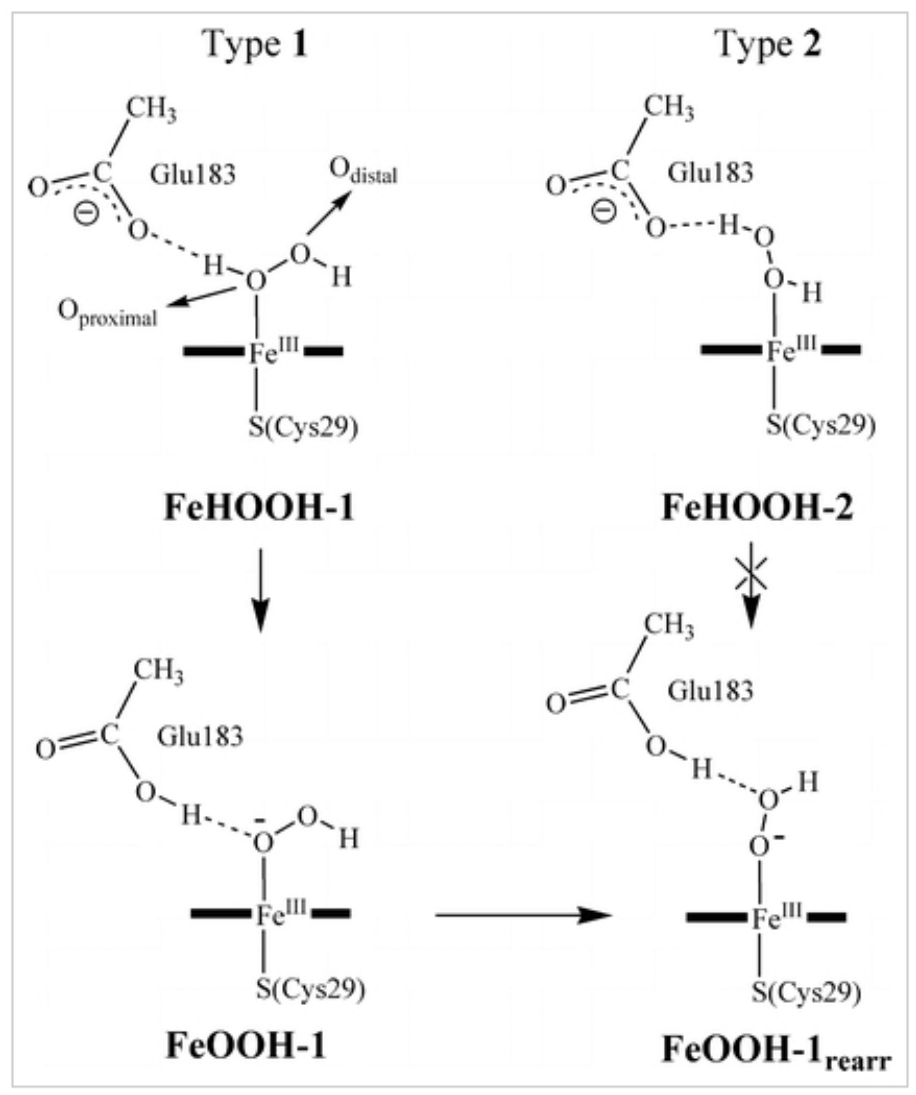

Figure 1.7. Mechanism of CPO Compound I formation, the horizontal bold line represents the porphyrin ring (105).

Compound $\mathrm{X}$ is an intermediate in the halogenation pathway and is also formed from Cpd I. In the synthetic enzyme model, Cpd X was formed upon addition of $\mathrm{Cl}^{-}$to $\mathrm{Cpd}$ I to produce a ferric $\mathrm{Fe}-\mathrm{OCl}^{-}$intermediate (109). Ferric $\mathrm{Fe}-\mathrm{OCl}^{-}$was unable to chlorinate monochlorodimedone (MCD), which is a common substrate used in chlorination reactions. In contrast, the protonated species Fe-HOCl converted MCD into dichlorodimedone (DCD). That explains the reactivity of $\mathrm{CPO}$ at a low $\mathrm{pH}$. The proposed reaction mechanism for chlorination reaction is shown in Figure 1.8 (110). 


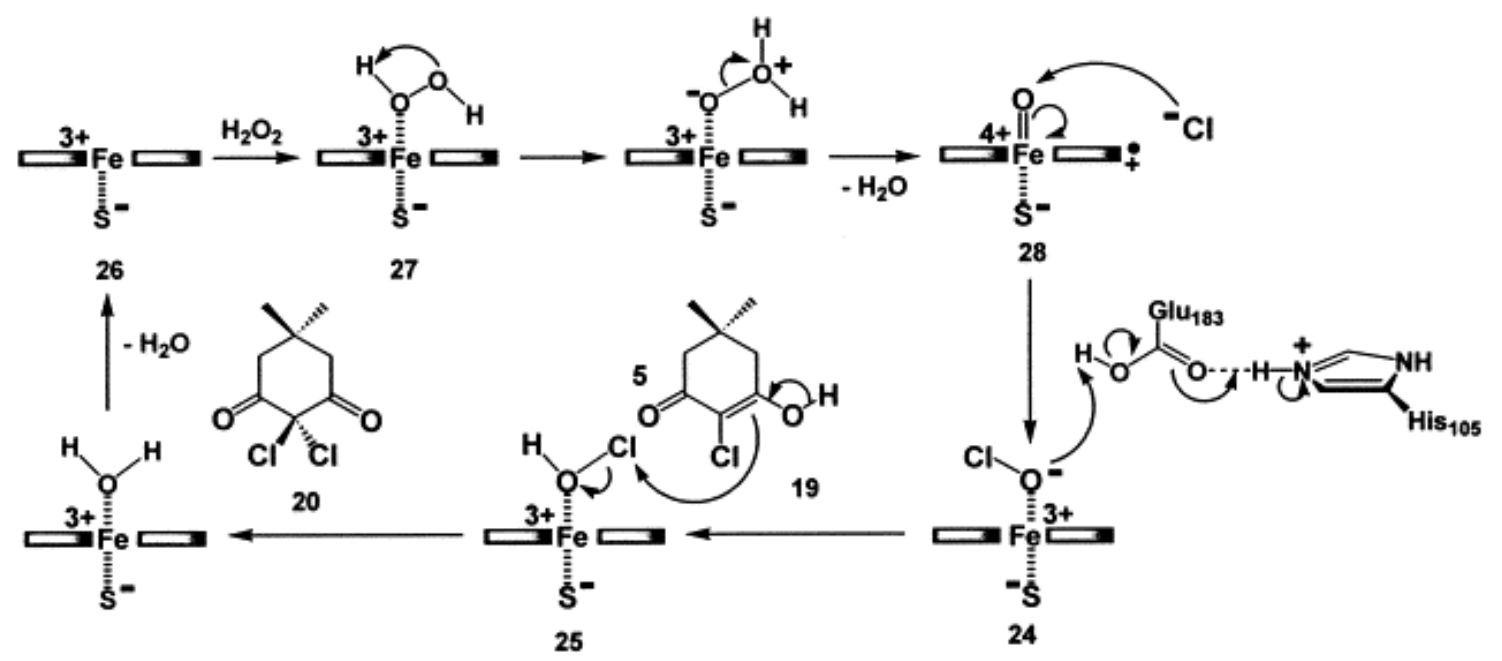

Figure 1.8. CPO-catalyzed chlorination mechanism (110).

\subsubsection{Reactions catalyzed by CPO}

As mentioned earlier, CPO is one of the most versatile heme enzymes, capable of catalyzing a plethora of oxidation reactions. One-electron oxidations occur via the peroxidation pathway while two-electron oxidations occur via the halogenation, oxygen insertion, and dismutation pathways (111-113). Chloroperoxidase is able to oxidize a variety of substrates including amines, alcohols, alkenes, esters, anisoles, sulfides, and indoles (111, 114-126).

\subsubsection{One-electron oxidations}

One-electron oxidations include peroxidation and oxidative de-halogenation reactions. Chloroperoxidase catalyzes peroxidations with less efficiency than classical peroxidases such as horseradish peroxidase and lignin peroxidase (127). Peroxidatic reactions occur on the surface of the enzyme (Fig. 1.9b) (112) via two one-electron transfer steps and involve formation of Cpd I and Cpd II intermediates (Reactions 1.1-1.4). In mechanisms of classical peroxidases (Fig 1.9), c was determined to be the rate-limiting 
step, while for CPO, the rates difference between b and c is smaller than that in other peroxidases (127).

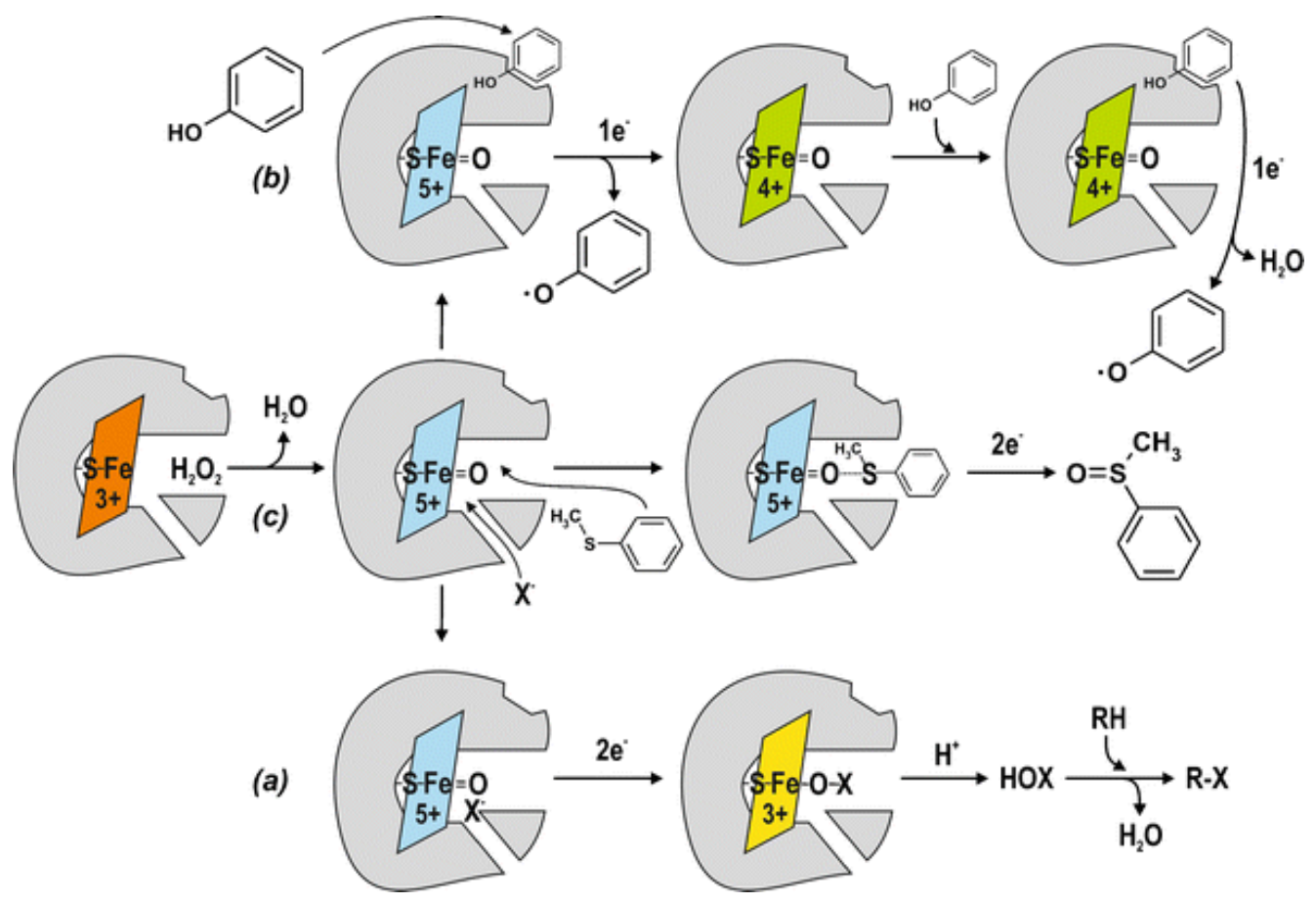

Figure 1.9. Mechanistic routes of different types of reactions catalyzed by CPO: (a) halogenation, (b) phenol oxidation, (c) sulfoxidation (113).
$2 \mathrm{R}-\mathrm{H}+\mathrm{H}_{2} \mathrm{O}_{2} \longrightarrow \mathrm{R}-\mathrm{R}+2 \mathrm{H}_{2} \mathrm{O}$
a. Enzyme $+\mathrm{H}_{2} \mathrm{O}_{2} \longrightarrow \mathrm{Cpd} \mathrm{I}+\mathrm{H}_{2} \mathrm{O}$
b. Cpd I + RH

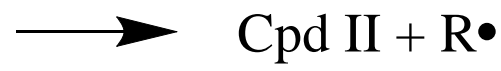
c. Cpd II + RH

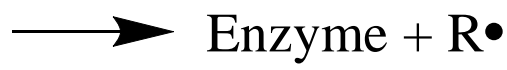

Peroxidation reactions are $\mathrm{pH}$-dependent with the highest rates achieved at acidic $\mathrm{pH}$ (128), which may indicate the requirement for Cpd II protonation and/or higher heme redox potential at low $\mathrm{pH}$. Typical substrates in one-electron reactions are aromatic hydrocarbons 
including ABTS (Fig. 1.10) and guaiacol that are commonly used to assay peroxidatic activities of enzymes $(129,130)$. Few data are available on the mechanism of peroxidation reactions catalyzed by $\mathrm{CPO}$, and there is an ongoing debate regarding the site at which oneelectron oxidations take place. Casella et al. reported that phenols possessing bulky substituents and bearing free amino groups were poor substrates for oxidation by CPO (131). In addition, CPO stereoselectively catalyzed the L-isomer of N-acetyltyrosine, but not the D-isomer. These results indicate that CPO's substrate selectivity is determined by size, charge, and the particular orientation of a molecule in the active site of the enzyme. Preference of smaller or para-substituted phenols as well as molecules lacking positive charge has been attributed to the rigidity of the access channel and the presence of positive amino acids in the active site, respectively. However, a more recent report by Manoj and Hager (112) argues that peroxidations most likely occur on the protein surface and not at the active site, on the basis of observed oxidations of bulky meta-substituted phenolics, benzylidene bis-dimethylaniline, and $\mathrm{N}^{\prime}, \mathrm{N}^{\prime}, \mathrm{N}^{\prime}, \mathrm{N}^{\prime}$-tetrabenzyl phenylenediamine, which are too big to fit into the active site of CPO. In addition, СPO treated with dialkyl pyrocarbonate is unable to perform reactions that require substrate binding to the active site, but retains its peroxidatic ability. Furthermore, different $\mathrm{pH}$ optima were reported for different substrates as well as for chloride-free and chloride-assisted peroxidations (132). These results suggest the possibility of two different peroxidation mechanisms and/or reaction sites.

Enzymatically catalyzed peroxidative reactions are of great interest to the public since they may be used to eliminate phenolic contaminants from water, degrade azo dyes utilized in textile industry, synthesize phenolic polymers as a healthier alternative to harmful 
phenol-formaldehyde methods $(133,134)$, remove lignin degradation products generated by wood decomposition for bio-fuel production, etc. $(135,136)$.<smiles>CCc1n/c(=N/N=c2\sc3ccc(S(=O)(=O)[O-])cc3s2)sc2cc(S(=O)(=O)O)ccc12</smiles>

ABTS<smiles>CCCCCCCCCCCC(=O)OO</smiles>

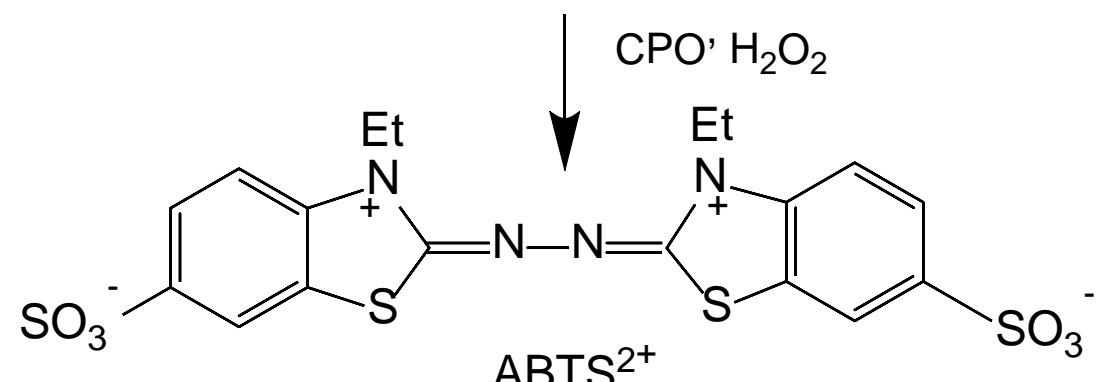

Figure 1.10. Oxidation of ABTS by CPO using hydrogen peroxide as an oxidant.

Another reaction that occurs via two one-electron transfer steps with Cpd I and Cpd II intermediates is the oxidative dehalogenation reported by Osborne et al. $(137,138)$ and further investigated by Murphy (139) and Diaz-Diaz (140). The substrates analyzed included trihalophenols and monofluorophenols (Fig. 1.11). In contrast to halogenation, where CPO is unable to use fluoride as a halogen source, CPO has been shown to catalyze defluorination. The discovery of this new reactivity of CPO has significant environmental implications. In particular, introduction of toxic chlorinated phenols into the environment 
by industrial processes is a growing concern and effective methods of phenol degradation are needed. Thus, CPO has a potential to be used in catalytic bioremediation of toxic compounds and help create healthier environment.

a)<smiles>[X]C1=CC(=O)C([X])C(=O)C1</smiles>

b)<smiles>Oc1ccc(F)cc1</smiles>

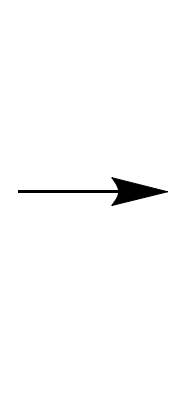<smiles>O=C1C=CC(=O)C=C1</smiles>

Figure 1.11. Dehalogenation of (a) trihalophenol, where $\mathrm{X}=\mathrm{F}^{-}, \mathrm{Cl}^{-}, \mathrm{Br}^{-}, \mathrm{I}^{-}$and (b) 4-fluorophenol.

\subsubsection{Two-electron oxidations}

Two-electron oxidations include a variety of reactions such as halogenations, peroxygenations, peroxide dismutations, and have been proposed to occur at the active site of CPO, with the exception of halogenations (Figs. 1.9a and 1.9c) $(112,113,141,142)$. The signature function of CPO is chlorination; however, it is also able to use bromide and iodide to halogenate various substrates as depicted in the reaction below, where $\mathrm{X}^{-}$includes $\mathrm{Cl}^{-}$, $\mathrm{Br}^{-}$, and $\mathrm{I}^{-}$:

$$
\mathrm{RH}+\mathrm{H}_{2} \mathrm{O}_{2}+\mathrm{X}^{-}+\mathrm{H}^{+} \longrightarrow \mathrm{R}-\mathrm{X}+2 \mathrm{H}_{2} \mathrm{O}
$$


Chloroperoxidase is unable to use hydrogen peroxide to oxidize fluoride ion since such a reaction is thermodynamically unfavorable (28). Halogenation involves formation of Cpd $\mathrm{X}$ and is most efficient at low $\mathrm{pH}$ with an optimum $\mathrm{pH}$ of 2.75 for $\mathrm{CPO}(132,143)$. It has been debated whether halogenations occur in the active site of CPO or via diffusible intermediate(s). Manoj investigated those two possibilities in chlorination reactions of 35 different substrates, and proposed that final chlorine transfer occurs outside the active site of CPO (141). This statement was derived from the observations that substrate size and topography had no significant effect on chlorination rates, and substrates larger that the active site dimensions were effectively chlorinated.

Substrates susceptible to halogenation by CPO include $\beta$-diketones, phenols (28), $\beta$ keto acids (27), tyrosine, thyroglobulin (144), alkenes (143), alkynes (118), and aromatic hydrocarbons (145). A few examples are shown in Figure 1.12. It should be noted that halogenation occurs non-stereoselectively (146) and with a difference in an intermediate formation between chlorination/bromination and iodination reactions. In the former two types of reactions there is no formation of halogen molecule while in the latter reaction iodide is oxidized into iodine intermediate $(28,143)$.

Other two-electron oxidations include oxygen insertions and other oxidative reactions. Oxygen insertions can be divided into epoxidations, sulfoxidations, and hydroxylations, which occur stereo- and regio-selectively $(124,147,148)$. Stereoselectivity arises from the fact that to gain access to CPO's active site, the substrates need to properly orient in the channel that connects the surface of the enzyme to the heme pocket. Substrates for these two-electron oxidations include amines, alcohols, aldehydes, alkenes, alkynes, esters, anisoles, sulfides and indoles (111-112, 115-126). 
a)<smiles>CCCCCCCCCCCCCC(=O)C1CC(=O)CC1=O</smiles>

b)<smiles>Oc1ccccc1Cl</smiles>

c)<smiles>[X]C(C)(C)C(C)(C)O</smiles>

d)<smiles>Clc1c2ccccc2c(Cl)c2ccccc12</smiles>

Figure 1.12. Examples of CPO-catalyzed halogenations : (a) 1,3cyclopentanedione, (b) phenol, (c) alkene, (d) anthracene (aromatic hydrocarbon).

Epoxidation reactions occur stereoselectively via oxygen transfer from hydrogen peroxide or organic peroxide to form epoxide products (34):
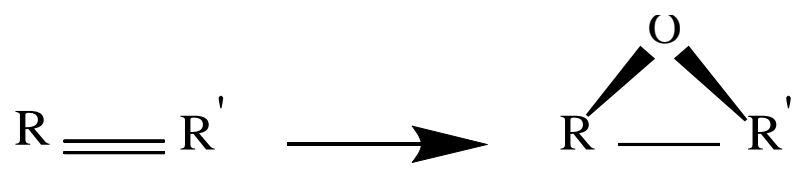

In $\mathrm{CPO}$, the nature of intermediates involved in epoxidation has not been elucidated yet; however, it has been suggested that epoxidation might occur via a dual pathway involving two different transition states (Fig. 1.13) (149). 
(a)

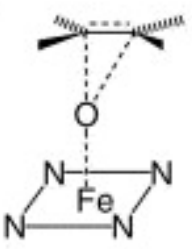

(b)

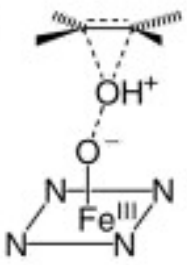

Figure 1.13. Possible transition states in epoxidation of cis-2-methylstyrene (149).

Chloroperoxidase catalyzes epoxidation of cis-disubstituted, 1,1-disubstituted, and tri-substituted alkenes that possess not more than 8 carbons, producing epoxides with the R-configuration as the major product. The highest enantiomeric excess and yields are obtained for cis-disubstituted olefins (Fig.1.14). Interestingly, trans-olefins and terminal alkenes were found to be poor substrates for CPO (147) as well as mono-substituted alkenes (150).

a)

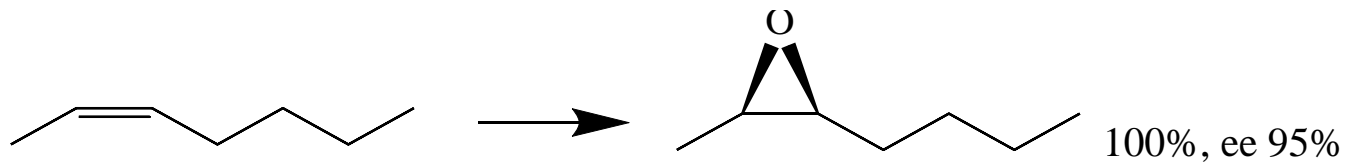

b)<smiles>C=C(C)CC(=O)OCC</smiles>

$67 \%$, ee $93 \%$

Figure 1.14. Stereoselective epoxidation of (a) cis-heptene and (b) 3-methyl-3butenoate with yield and enantiomeric excess (ee) percentages.

Chiral epoxides are of great interest to pharmaceutical and chemical industries since they can be used as synthetic precursors to drugs and natural products (151). Currently, enantiopure drugs can be synthesized by high-cost multi-step organic methods which produce large amounts of toxic by-products and low yields of the compounds of interest $(128,152)$. Non-toxic, environment-friendly synthesis via enzymatic catalysis is thus a very promising alternative; however, there are some difficulties associated with the largescale utilization of CPO for epoxide production. 
One of the challenges in epoxidation reactions is determining the appropriate amount of the hydrogen peroxide oxidant to be used since large amounts of the oxidant cause oxidative damage to $\mathrm{CPO}$. Another issue is the hydrogen peroxide dismutation ability of СРО (Eq. 1.7), which depletes the oxidant before significant yields of the desired product is produced. Decreased product yield is the result of competition between hydrogen peroxide and the desired substrate for the particular reaction with Cpd I (153). Sometimes, other commonly used oxidants such as iodosobenzene and tert-butyl hydroperoxide can be substituted for hydrogen peroxide as is the case with functionalized cis-olefins (121). However, many simple unfunctionalized cis-alkenes require $\mathrm{H}_{2} \mathrm{O}_{2}$ for the epoxidation to occur (147).

$$
2 \mathrm{H}_{2} \mathrm{O}_{2} \longrightarrow \mathrm{O}_{2}+2 \mathrm{H}_{2} \mathrm{O}
$$

Despite difficulties of epoxidations, there are several examples of successful applications of CPO in the production of special pharmaceutical compounds including synthesis of R-(-)-mevalonolactone (154) and (R)-dimethyl 2-methylaziridine-1,2dicarboxylate (155) that are important precursors for pentanoid compounds and alphamethylamino acids, respectively.

Another two-electron oxidation catalyzed by CPO is the stereoselective sulfoxidation of organosulfides (Eq. 1.8), producing mainly R-sulfoxides (142, 156-158):

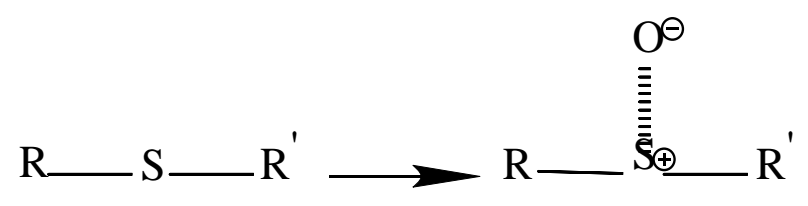

Several substrates such as aromatic and $\beta$-carbonyl sulfides (157-160) were studied by different research teams. As with the epoxidation reaction, the size and nature of substrates 
as well as the choice of the oxidant play an important role in product yield and enantiomeric excess (Fig. 1.15) $(148,160)$. Bulky substrates are either poorly oxidized or not oxidized at all. Para-substituted aryl alkyl sulfides or sulfides containing electron-donating groups on the benzene ring are more easily oxidized than ortho-substituted sulfides or sulfides containing electron-withdrawing groups. As for the oxidants, tert-butyl hydroperoxide and hydrogen peroxide are more effective than other oxidants in sulfide oxidations, giving increased product yields and enantioselectivity (157). Additionally, the manner in which the oxidant is introduced into the reaction system is critical for obtaining high yields of enantiopure products. The concentration of the oxidant must be kept low to prevent $\mathrm{H}_{2} \mathrm{O}_{2}$ accumulation, which hinders the reaction by inactivating the enzyme. A low concentration of the oxidant can be achieved by continuously adding the oxidant at the rate of the initial reaction (125).

Many CPO-catalyzed sulfoxidations have environmental and biomedical significance. One of the examples of this utility is the removal of organosulfur from diesel fuel to reduce harmful effects on the environment. Chloroperoxidase converts organosulfides into the corresponding sulfones and sulfoxides, which can be effectively eliminated by distillation $(152,161)$. Another example is CPO-catalyzed synthesis of $(R)$-modafinil, which is used to treat sleep disorders and narcolepsy (162).

The next type of oxidation to be discussed will be hydroxylation, which can be stereoselective or nonstereoselective, depending on reaction conditions. Additionally, hydroxylation most likely occurs via the oxygen insertion pathway as determined by Zaks and Dodds (117). A dual pathway for CPO-catalyzed hydroxylations was proposed based on hydroxylations catalyzed by P450s (Fig. 1.16) (149). The first one involves an oxo- 
ferryl species with transition state $\mathbf{2 0}$ while the other one involves ferric hydroperoxide species with transition state 21 (Fig. 1.16). However, the mechanism of hydroxylation remains to be elucidated.

a)<smiles>CCCCCCCCC(C)=O</smiles>

b)<smiles>CCSCC(=O)c1ccc(C)cc1</smiles>
$100 \%$, ee $99 \%$

c)<smiles>CSc1ccc(C)cc1</smiles>
$31 \%$, ee $92 \%$

d)

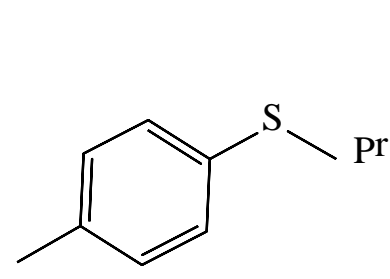

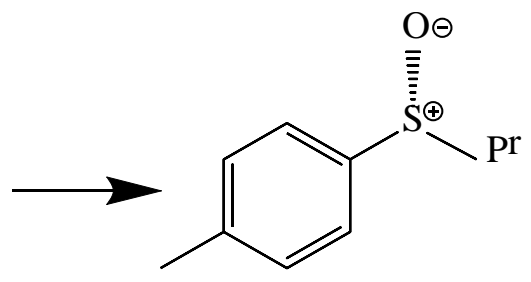
$98 \%$, ee $91 \%$

e)<smiles>CSc1ccc([N+](=O)[O-])cc1</smiles>

Figure 1.15. Stereoselective sulfoxidation of (a,b) beta-carbonyl sulfides and (c,d,e) aryl sulfides with yield and enantiomeric excess (ee) percentages. The nature and size of the substrate influence the yield and ee values. 


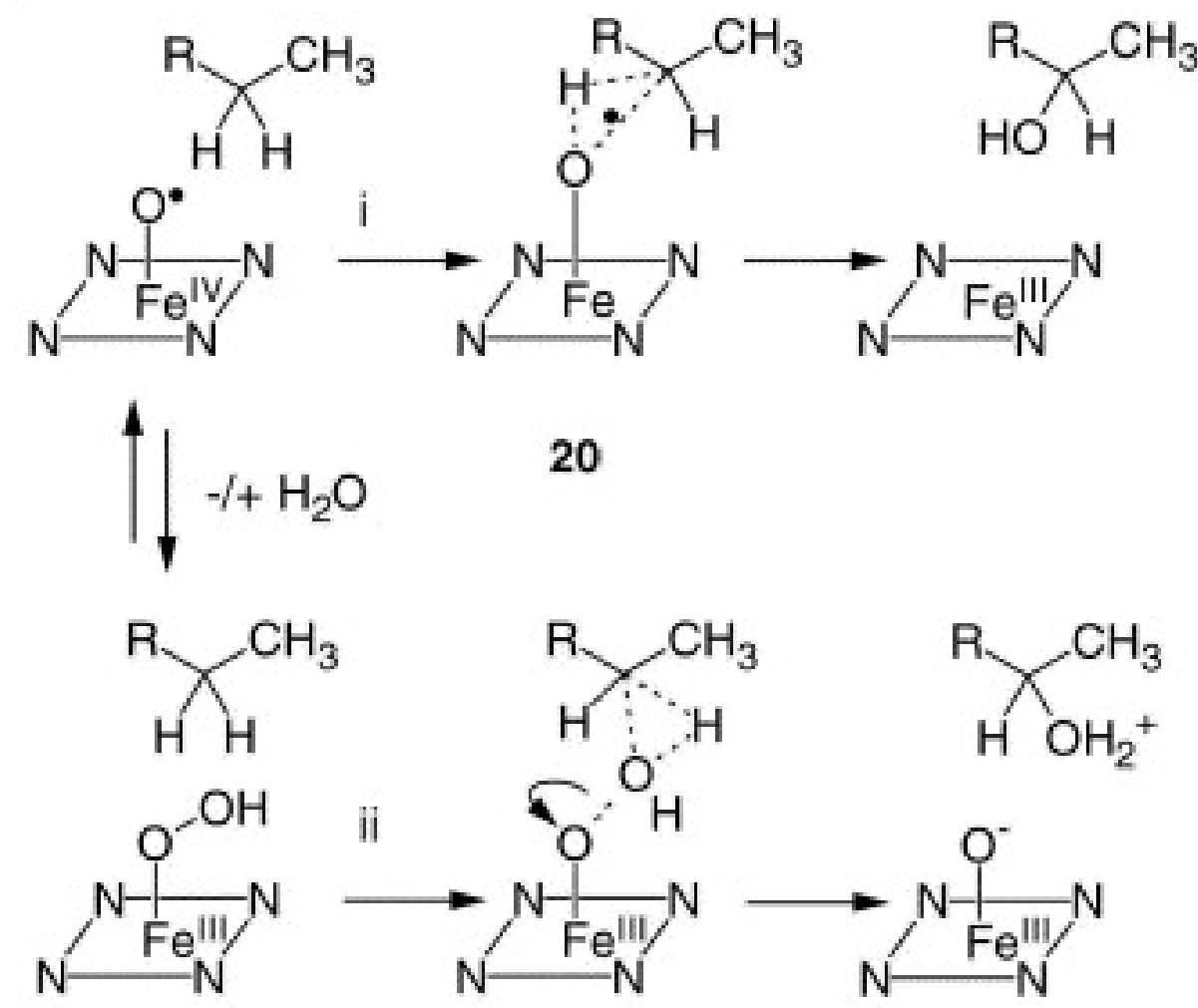

21

Figure 1.16. Dual mechanism proposed for CPO-catalyzed hydroxylations (149).

As with other oxidation reactions, the oxygen in hydroxylation comes from an oxidant such as hydrogen peroxide (124). In contrast with epoxidation and sulfoxidation reactions, hydroxylation products can have either R (2-phenethyl alcohol) or S (1-phenyl-1-propanol) configuration (117). Among the substrates of CPO that undergo hydroxylation are pmethylanisole and p-ethylanisole (124), functionalized and unfunctionalized acetylenes (121), cyclic conjugated dienes $(163,164)$, and $\mathrm{R}-(+)-$ limonene that belongs to monoterpenes (165). As with aryl sulfides, benzylic compounds containing orthosubstitutions are poorly hydroxylated or not hydroxylated at all. The degree to which the reaction proceeds is determined by substrate oxidation potential, nature of substituents, and steric requirements (124). 
The stereoselectivity of CPO-catalyzed hydroxylation is dependent on the nature of the substrate. In reactions with 1,3-cyclohexadienes, the product of the reaction with 2hydroxyphenol has an enantiomeric excess of $78 \%$ while 1,3 -cyclohexadiene diacetate is converted into a racemic mixture (Fig. 1.17) (163). The presence of chloride in the reaction may influence the enantiomeric excess as observed with the R-(+)-limonene, which is converted into a single enantiomer (99\%) when there is no chloride, while a racemic product is formed if chloride is present (Fig. 1.18) (165). The loss of stereoselectivity when chloride is present suggests that chloride ions may be converted to hypochlorite, which in turn oxidizes limonene without stereoselectivity. Thus, CPO-catalyzed hydroxylations are less stereoselective than epoxidations or sulfoxidations. However, with carefully controlled conditions, useful enantiopure products can be obtained, such as during halo-hydroxylation of chloropropene and allyl alcohol to prepare chiral precursors for agrochemical and pharmaceutical industries (166). The side reactions that need to be controlled include further oxidation of alcohols to aldehydes and oxidant dismutation (122).

In addition to the reactions described above, $\mathrm{CPO}$ is able to catalyze the oxidation of different monoterpenes in the presence of halide to yield halohydrins (167), regio- and stereoselective bromohydration of glycals to produce 2-deoxy-2-bromo saccharides (168), oxidation of unsubstituted and substituted indoles to yield oxindoles $(125,169,170)$, Noxidation of arylamines to give nitroso metabolites $(111,169,171)$, oxidation of alcohols to aldehydes (116), and oxidation of conjugated dienoic esters to yield a variety of products (123). Many of the above compounds can be converted into chemicals that serve as intermediates in the synthesis of flavors, fragrances, and drugs $(125,167)$. 
a)<smiles>O[C@H]1C=CC=C[C@@H]1O</smiles><smiles>O[C@H]1C=C[C@@H](O)[C@H](O)[C@H]1O</smiles>

$25 \%$, ee $78 \%$

b)

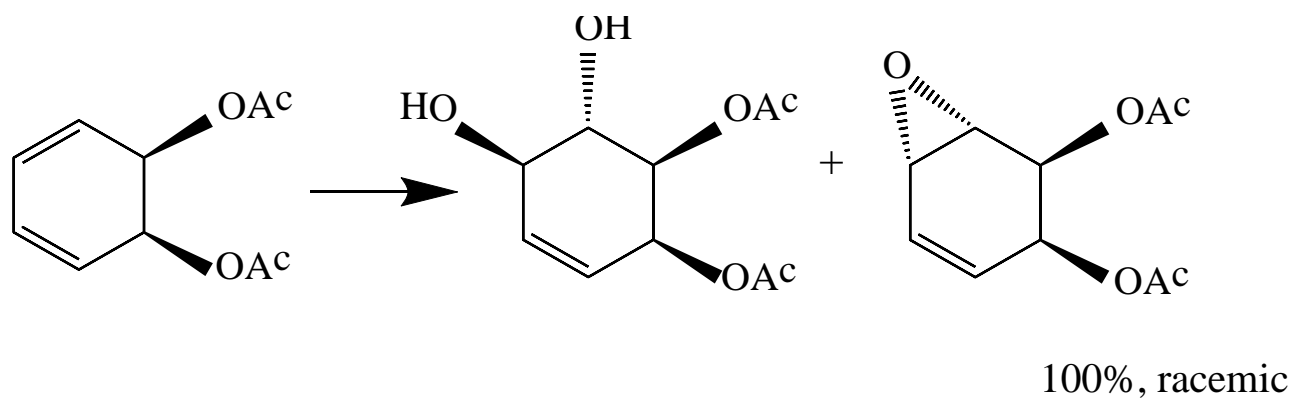

Figure 1.17. Hydroxylation of 1,3-cyclohexadienes catalyzed by CPO. The nature and size of the substrate influence the yield and ee values.

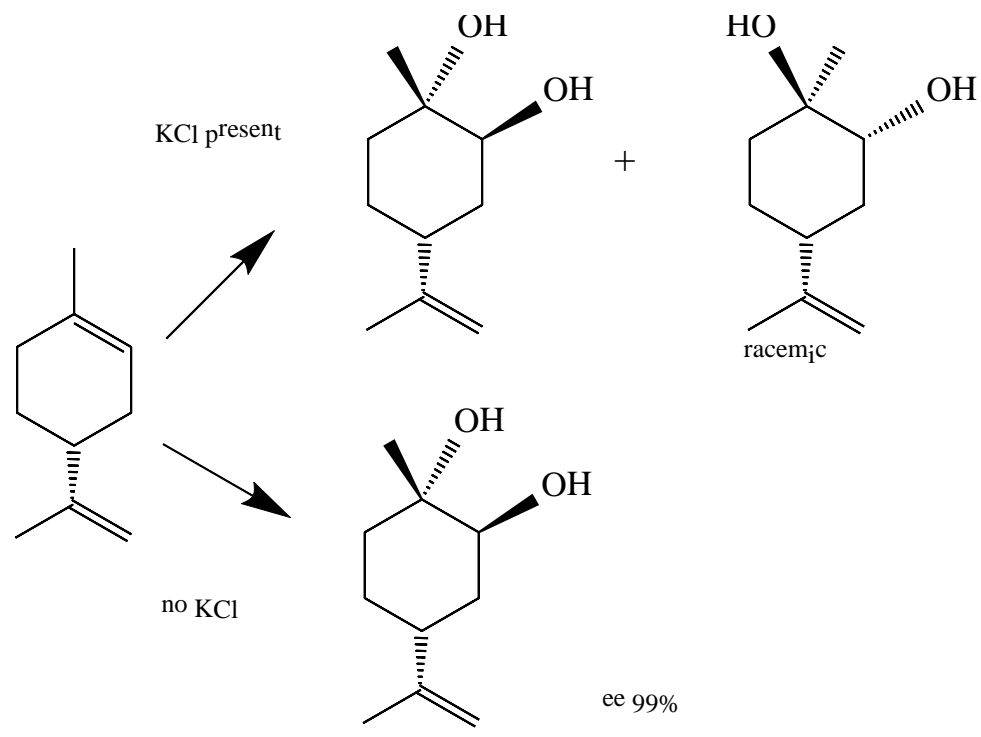

Figure 1.18. Oxidation of R-(+)-limonene catalyzed by CPO in the presence and absence of $\mathrm{KCl}$.

\subsubsection{Ligand-binding properties}

Despite similarities between spectroscopic properties of CPO and P-450cam (172), in terms of ligand-binding properties, CPO has a closer relationship to peroxidases. Many 
ligands except for the halides, acetate, and nitrate, form low-spin complexes with CPO (173, 174). Since weakly acidic ligands with pKa 3.2-9.14 (cyanide, pyridine, imidazole, fluoride) bind to ferric $\mathrm{CPO}$ in their neutral form and lose their proton upon ligation, the binding affinity is decreased above their pKa. In contrast, very acidic ligands with negative pKa (thiocyanate, nitrate, chloride, bromide, and iodide) seem to bind in their anionic form and display varying affinities in $\mathrm{pH}$ range 2-7. For neutral ligands such as carbon monoxide and $n$-butyl isocyanide, no changes in affinity were observed at various $\mathrm{pH}$. It has been suggested that for ligand binding to ferric CPO, an amino acid with a pKa less than 2 is involved, while a group with the pKa of 5.5 is involved in ligand binding to ferrous form of the enzyme (174).

Carbon monoxide and cyanide have high affinities for $\mathrm{CPO}$ and most heme proteins in general, and make open crevice structures more stable (175). Interestingly, when diatomic and triatomic ligands bind in the distal site of $\mathrm{CPO}$, they do so without requiring movement of the distal residues to accommodate those ligands (176). Instead, the distal Glu 183 interacts with ligands either electrostatically or via hydrogen bonding. This becomes very important during the formation of Cpd I when hydrogen peroxide binds to the heme, and Glu 183 acts as an acid-base catalyst to promote O-O scission and release of water (176).

\subsection{Spectroscopic properties of heme proteins}

Electronic absorption, resonance Raman (RR), nuclear magnetic resonance (NMR), and circular dichroism (CD) spectroscopies are frequently used to investigate heme proteins. Electronic absorption spectroscopy or UV-Vis spectrophotometry, can be used to investigate electronic structure of heme and its environment, i. e., the coordination and spin 
state of the heme iron. A typical absorbance spectrum of a heme protein (Fig. 1.19) (177) includes a peak at around $400 \mathrm{~nm}$ called the Soret band and minor peaks in 500-700 nm region called Q bands that result from transitions in the porphyrin (Fig. 1.20) (178).

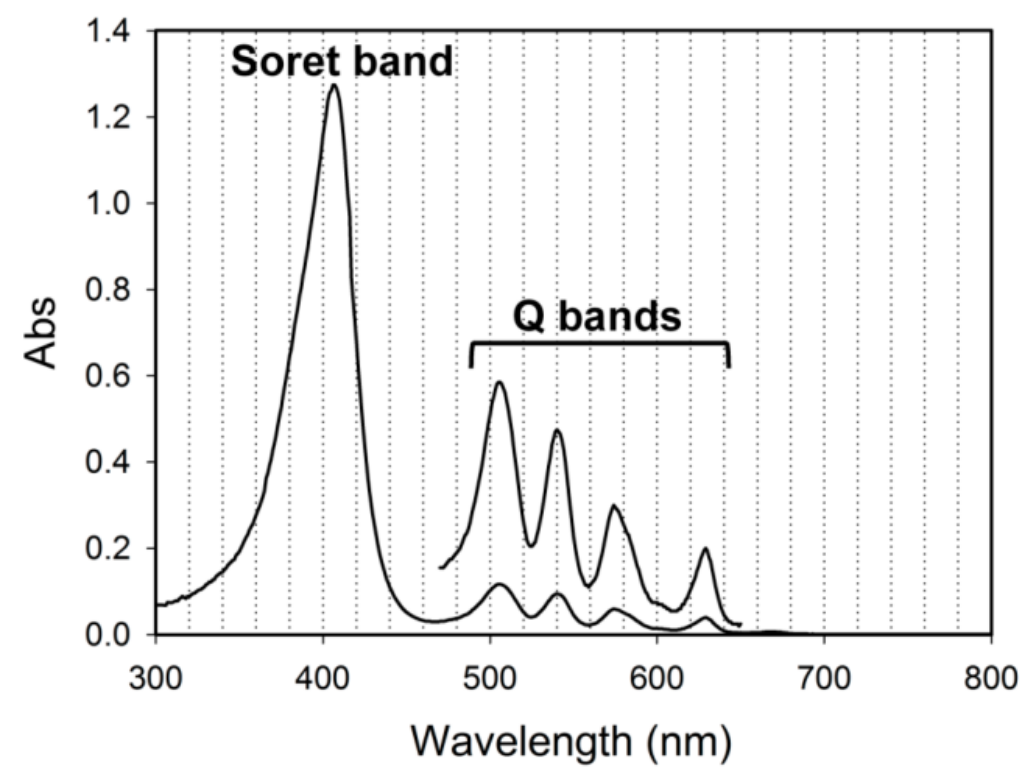

Figure 1.19. A typical electronic absorption spectrum of a heme protein (177).

The Soret and Q bands both arise from $\pi-\pi^{*}$ transitions and can be explained using the Gouterman four orbital model that was proposed in the early 1960s (178). According to this model, transitions between two highest occupied molecular orbitals (HOMOs) and two lowest unoccupied molecular orbitals (LUMOs) produce these absorption bands and their relative energies depend on the substituents on the porphyrin ring and the identity of the metal center. The Soret peak is a result of a strong transition to the second excited state (S2) while minor peaks are the result of weak transitions to the first excited state (S1). Furthermore, the $\mathrm{Q}$ band that results from a transition from the resting state to the $\mathrm{S} 1$ vibrational ground state is called the $\alpha$ band while the $\mathrm{Q}$ band due to transitions to higher vibrational levels in the S1 is called the $\beta$ band and appears at slightly lower wavelengths 
than the $\alpha$ band. The intensities of the $\alpha$ and $\beta$ bands relative to each other can serve as a measure of how close in energy the $\mathrm{a}_{2 u}$ and $\mathrm{a}_{1 \mathrm{u}}$ orbitals are. For instance, if both orbitals are very similar in energy, the degree of orbital mixing will be large. In this case, the $\alpha$ band intensity will be small and the $\alpha / \beta$ intensity ratio will be small as well. On the contrary, if the $\mathrm{a}_{2 u}$ and $\mathrm{a}_{1 \mathrm{u}}$ orbitals have very different energies, the $\alpha / \beta$ intensity ratio will be larger because of the smaller degree of orbital mixing (179).

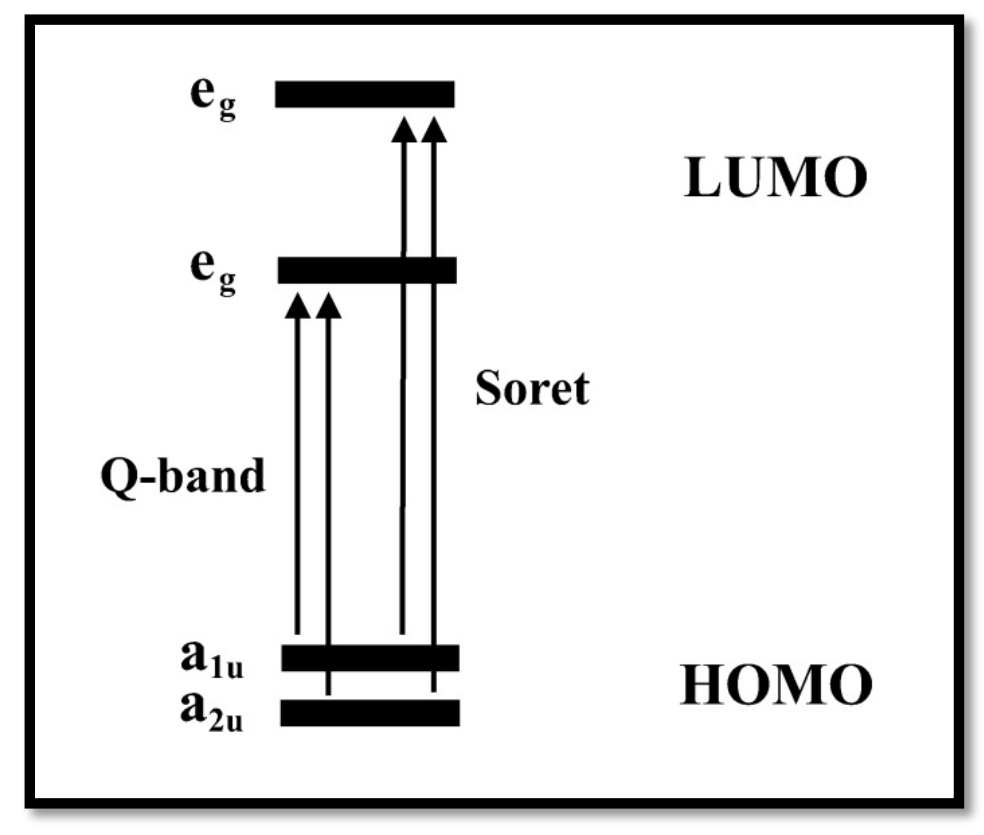

Figure 1.20. The Gouterman four orbital model.

Other interactions that can be observed with UV-Vis spectrophotometry include porphyrin - iron interactions. Since ferrous iron has filled $d \pi$ orbitals $\left(\mathrm{d}^{6}\right)$, ferrous heme is a hypsoporphyrin in which the interactions between iron $\mathrm{d} \pi(\mathrm{d}(\mathrm{xz}), \mathrm{d}(\mathrm{yz}))$ and porphyrin $\pi^{*}\left(\mathrm{a}_{1 \mathrm{u}}\right)$ orbitals are significantly stronger. These strong interactions result in a larger porphyrin $\pi$ to $\pi^{*}$ ( $\mathrm{a}_{2 \mathrm{u}}$ to $\mathrm{a}_{1 \mathrm{u}}$ ) energy difference, causing the hypsochromic (blue) shifts in the electronic spectra (Fig. 1.21). In contrast, in regular metalloporphyrins with closed dshells $\left(\mathrm{d}^{0}\right.$ or $\mathrm{d}^{10}$ ), the $\mathrm{d} \pi$ metal orbitals have little effect on the $\pi$ to $\pi^{*}$ energy separation in 
porphyrin electronic spectra because of their low energy (180).

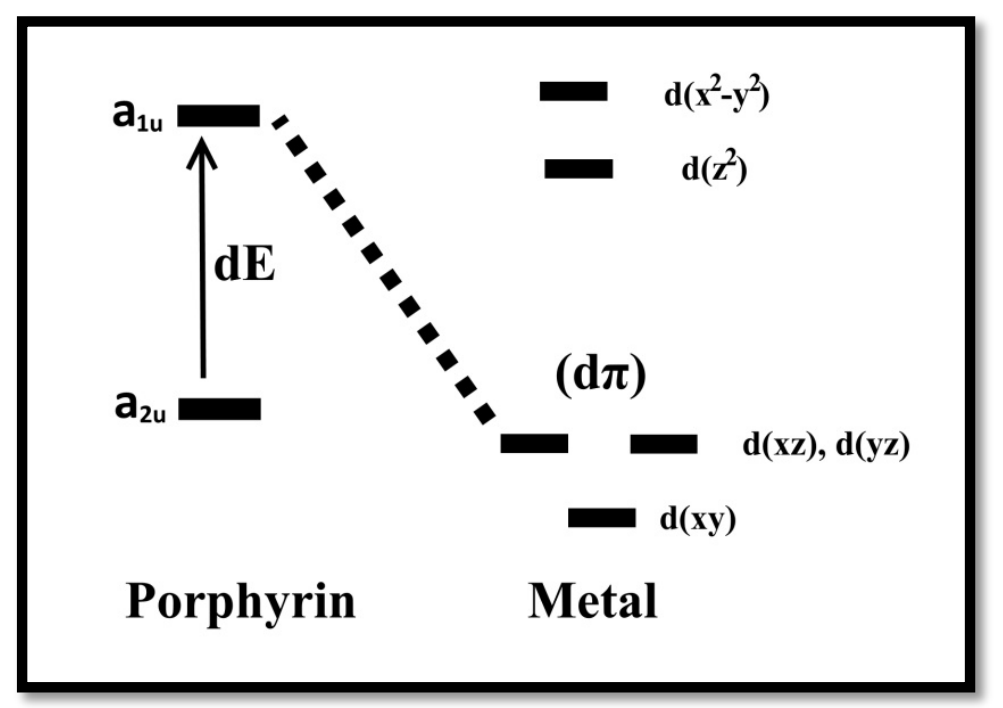

Figure 1.21. Interactions between iron $d \pi(d(x z), d(y z))$ and porphyrin $\pi^{*}\left(a_{1 u}\right)$ orbitals. Resonance Raman spectroscopy is used to monitor structural changes in the heme upon ligand binding and electron transfer (181). In the Raman spectrum of a heme protein, several bands in the high-frequency region are associated with vibrations of porphyrin substituents while bands in the low-frequency region arise from vibrations of the axial ligands. Thus, resonance Raman spectroscopy can be used to investigate the structure of a heme center, as well as the spin of the iron and the nature of the proximal and distal ligands (182).

Proton NMR is another powerful technique that allows structural characterization of hemoproteins. Although it has been challenging to assign all heme protons in the NMR spectrum for CPO, it is a useful technique for verifying the proximal ligand substitutions, as well as assessing other important residues in the active site of CPO (183). The broad resonances make it difficult to analyze a proton NMR spectrum of a native high-spin heme protein. Thus, the cyanide-bound low-spin protein complex may be analyzed to obtain 
information on electronic and molecular structural properties.

Circular dichroism can be utilized in the determination of protein alpha-helical and beta-sheet content as well as for monitoring of secondary and tertiary structural changes in mutant proteins or protein-ligand complexes. Several programs, such as CONTINLL (184, 185), SELCON3 $(186,187)$, CDSSTR $(188-190)$, VARSLC $(188,189)$, and K2D (191) are available for estimation of the protein secondary structures. The algorithms differ in the number and nature of the reference protein sets used in analysis, thus one method might be more suitable than others for a particular protein. As for tertiary structure CD, it is useful for confirming the integrity of protein domains expressed separately, investigating protein stability under changing conditions, and observing structural alterations in mutants or upon ligand binding. For heme proteins, CD may also provide information on changes in the environment of the heme group (192). Additionally, it is possible to assign a protein to a particular class (all alpha, all beta, alpha+beta, alpha/beta, denatured) based on the method developed by Venyanimov and Vassilenko (193), which is especially useful for new understudied proteins.

\subsection{Site-directed mutagenesis to study structure-function relationships in heme proteins}

Heme enzymes are attractive targets for protein engineering because of their catalytic diversity. There are four approaches to constructing heme enzymes with desired catalytic and substrate-binding properties: modification of the heme group, exon shuffling of hemoglobin, directed evolution, and rational design (194). In my study, I employ the latter approach using site-directed mutagenesis to explore how changes in the proximal active site affect biochemical properties of CPO. Many heme protein mutants have been prepared 
using site-directed mutagenesis to gain insights into the role of the heme environment in catalysis and substrate specificity. The results of several of those studies are summarized in Table 1.3.

Table 1.3. Effects of distal and proximal active site mutations on catalytic activities of some heme proteins.

\begin{tabular}{|c|c|c|}
\hline Protein/Mutation & Activity Changes & References \\
\hline \multicolumn{3}{|l|}{$M b$} \\
\hline $\begin{array}{l}\text { F43H/H64L } \\
\text { (distal His relocation) }\end{array}$ & $\begin{array}{l}\text { Increased peroxidase, epoxidase, and } \\
\text { sulfoxidase activities, increase in heme } \\
\text { iron-distal His distance from } 4.3 \AA \text { to } 5.7 \AA \\
\text { (similar to peroxidases) }\end{array}$ & (195) \\
\hline $\begin{array}{l}\mathrm{L} 29 \mathrm{H} / \mathrm{H} 64 \mathrm{~L} \\
\text { (distal His relocation) }\end{array}$ & $\begin{array}{l}\text { Decreased peroxidase activity, increase in } \\
\text { heme iron-distal His distance from } 4.3 \AA \\
\text { to } 6.6 \AA \text { (too far for efficient } \mathrm{H}_{2} \mathrm{O}_{2} \\
\text { activation) }\end{array}$ & $(195)$ \\
\hline $\begin{array}{l}\text { H64D } \\
\text { (distal His substitution) }\end{array}$ & $\begin{array}{l}\text { Increased peroxidase activity, } \\
\text { replacement with aspartate that can act as } \\
\text { acid-base catalyst in formarion of Cpd I }\end{array}$ & $(195)$ \\
\hline $\begin{array}{l}\text { H93Y } \\
\text { (proximal His substitution) }\end{array}$ & $\begin{array}{l}\text { Decrease in catalase activity, possibly } \\
\text { poor } \mathrm{H}_{2} \mathrm{O}_{2} \text { binding }\end{array}$ & (196) \\
\hline \multicolumn{3}{|l|}{ HRP } \\
\hline $\begin{array}{l}\mathrm{R} 38 \mathrm{H}, \mathrm{R} 38 \mathrm{H} / \mathrm{H} 42 \mathrm{~V} \\
\text { (distal His relocation) }\end{array}$ & $\begin{array}{l}\text { Decreased peroxidase activity, but } \\
\text { increased sulfoxidation and epoxidation, } \\
\text { better access to the active site }\end{array}$ & $(197)$ \\
\hline $\begin{array}{l}\text { H42E, H42Q } \\
\text { (distal His substitution) }\end{array}$ & $\begin{array}{l}\text { Decreased peroxidase activity, } \\
\text { suboptimal orientation of E lacking H- } \\
\text { bonding with N70 }\end{array}$ & $(198)$ \\
\hline $\begin{array}{l}\text { F41A } \\
\text { (increase in heme access) }\end{array}$ & $\begin{array}{l}\text { Similar peroxidase activity to wild-type } \\
\text { (WT), increased sulfoxidation and } \\
\text { epoxidation }\end{array}$ & (199) \\
\hline N70V, N70D & & $(200)$ \\
\hline
\end{tabular}




\begin{tabular}{|c|c|c|}
\hline (H-bond disruption) & $\begin{array}{l}\text { Decreased peroxidation, deletion of } \mathrm{H}- \\
\text { bonding between } \mathrm{H} 42 \text { and N70 }\end{array}$ & \\
\hline \multicolumn{3}{|l|}{ CcP } \\
\hline $\begin{array}{l}\text { W51H/H52L } \\
\text { W51H/H52W } \\
\text { (distal His relocation) }\end{array}$ & $\begin{array}{l}\text { Decreased catalytic activity because of the } \\
\text { slower reaction with } \mathrm{H}_{2} \mathrm{O}_{2}\end{array}$ & $(201)$ \\
\hline $\begin{array}{l}\text { H52D, H52E, H52N, H52Q } \\
\text { (distal His substitution) }\end{array}$ & $\begin{array}{l}\text { Decreased activity because of the slower } \\
\text { rates of Cpd I formation }\end{array}$ & (202) \\
\hline $\begin{array}{l}\text { H175E } \\
\text { (proximal His substitution) }\end{array}$ & Increased activity & $(106)$ \\
\hline \multicolumn{3}{|l|}{ P450cam } \\
\hline $\begin{array}{l}\text { T96A, T96F } \\
\text { (distal Tyr substitution) }\end{array}$ & Increased hydroxylation & (203) \\
\hline C357U & Decreased oxygenase activity & $(204)$ \\
\hline \multicolumn{3}{|l|}{ P450175A1 } \\
\hline $\begin{array}{l}\text { L80H, L80Q } \\
\text { (distal Leu substitution) }\end{array}$ & $\begin{array}{l}\text { Increased peroxidase activity } \\
\text { (pH dependence, increase in basicity of } \mathrm{H} \\
\text { and Q and pKa increase of peroxide more } \\
\text { active at basic } \mathrm{pH} \text { ) }\end{array}$ & $(205)$ \\
\hline \multicolumn{3}{|l|}{ CPO } \\
\hline $\begin{array}{l}\text { E183H } \\
\text { (distal Glu substitution) }\end{array}$ & $\begin{array}{l}\text { Decreased chlorination and dismutation, } \\
\text { increased epoxidation. }\end{array}$ & (206) \\
\hline
\end{tabular}

From the data above, it is evident that there are multiple factors that regulate redox potentials and catalytic activities of heme enzymes. These factors include the nature of the proximal ligand, the heme iron-ligand distance, the nature of a distal acid-base catalyst, hydrogen bonding networks in both distal and proximal heme pockets, polarity of the active site, and the accessibility of the active site by substrates.

To better understand structure-function relationships in this class of proteins, several mutagenesis studies were performed on such heme proteins as myoglobin, horseradish 
peroxidase, and cytochrome $c$ peroxidases. Despite the fact that CPO from C. fumago was discovered in 1966, it is still poorly understood how CPO directs catalysis of such a broad range of reactions and substrates. There is much debate about the importance of the proximal thiolate ligation in $\mathrm{CPO}$ as well as contributions of other structural features in defining biochemical properties of this enzyme.

In my work, I utilized site-directed mutagenesis to introduce single or double amino acid substitutions in the active site of $\mathrm{CPO}$ in order to explore their roles in the structure and catalysis of this protein. I concentrated my attention on the proximal region of the heme pocket, and investigated the importance of Cys 29 as well as the hydrogen bond network between Arg 26 - Asn 37 and Ala 27 - Asn 33 for biochemical properties of CPO. Since there is no consensus on the role of Cys 29 in CPO catalysis, I chose to substitute Cys 29 with a His to imitate proximal ligation of a classical peroxidase and investigate the properties of the mutant. Furthermore, I replaced Arg 26 and Asn 33 with Ala in R26A, N33A, and R26A/N33A mutants to disrupt the hydrogen bond network. It is reasonable to suggest that these hydrogen bonds are important for maintaining the particular tertiary structure of CPO, and possibly, for catalysis. Since no data are available on the role of hydrogen bonds in the proximal pocket of CPO, I chose to investigate how removal of some or all hydrogen bonds in this network would affect the biochemical properties of this enzyme. The following chapters will present my findings on the characterization of $\mathrm{C} 29 \mathrm{H}$, C29H/C79H, C29H/C79H/C87H, R26A, N33A, and R26A/N33A CPO mutants, compare their biochemical properties to those of WT CPO, and discuss factors that play a significant role in the structure and function of CPO. 


\section{CHAPTER II.}

\section{THE ROLE OF THE PROXIMAL LIGAND IN CPO, A LONG LASTING \\ DEBATE}

\subsection{Background}

In heme proteins, the nature of the axial ligand together with active site polarity and electrostatic interactions influence the heme redox potential, which in turn, affects reactivity (207). Common proximal ligands include histidine (most peroxidases), cysteine (CPO, UPOs, and P450s), and tyrosine (catalases) $(49,63,64,67,71)$. The role of the proximal thiolate ligand in defining the biochemical properties of heme proteins has been much debated $(48,172,208-210)$. It has been suggested that the heme proximal ligation to a cysteine is responsible for the Soret peak near $450 \mathrm{~nm}$ for ferrous CPO - carbon monoxide (CO) complex, similarly to CO-bound P450 (172, 208). By comparison, carbon monoxide complexes of heme proteins with His as a proximal ligand absorb near $420 \mathrm{~nm}(106,196)$. In addition, it has been postulated that thiolate ligand plays a crucial role in the catalytic diversity of CPO and provides it with the ability to perform chlorination reactions, while other peroxidases with the imidazole proximal ligation either lack or have a very low level of chloride oxidation ability $(48,209,210)$.

Many site-directed mutagenesis studies have been done on heme proteins with imidazole and thiolate proximal ligation to understand the role of the proximal ligand in catalysis of heme proteins and the results of those studies are summarized in Table 2.1. From the data in Table 2.1, it is evident that substitutions of native proximal ligands dramatically change the catalytic abilities of heme proteins. However, it is difficult to clearly define ligand-function relationships. For example, upon substitution of the proximal 
His with a Cys in myoglobin, oxygen insertion reaction rates are increased $(196,211)$. However, similar substitution in $\mathrm{C} c \mathrm{P}$ results in decreased overall activity of the enzyme (106). Furthermore, replacement of the proximal Cys with a His in P450s results in a complete loss of oxygenase activity (212-214). However, similar substitution in CPO results in only slightly decreased epoxidation rates (215).

Table 2.1. Effects of proximal active site mutations on catalytic activities of some heme proteins.

\begin{tabular}{|c|c|c|}
\hline Protein/Mutation & Activity Changes & References \\
\hline \multicolumn{3}{|l|}{$M b$} \\
\hline H93С & $\begin{array}{l}\text { Increased sulfoxidation and epoxidation } \\
\text { rates, decreased dismutation }\end{array}$ & $(196,211)$ \\
\hline $\begin{array}{l}\text { H64G/H93C, } \\
\text { H64V/H93C }\end{array}$ & $\begin{array}{l}\text { Slightly decreased sulfoxidation rates } \\
\text { because of the hydrophobic distal site }\end{array}$ & $(211)$ \\
\hline H93Y & $\begin{array}{l}\text { Decrease in catalase activity possibly } \\
\text { because of the poor } \mathrm{H}_{2} \mathrm{O}_{2} \text { binding }\end{array}$ & $(196)$ \\
\hline \multicolumn{3}{|c|}{$\mathrm{S}_{2}$} \\
\hline H170A & Loss of peroxidase activity & $(199)$ \\
\hline \multicolumn{3}{|l|}{ CcP } \\
\hline H175Q & Similar activity to WT & $(106)$ \\
\hline H175E & Increased activity & $(106)$ \\
\hline H175C & Significant decrease in activity & $(106)$ \\
\hline \multicolumn{3}{|l|}{ P450 2B4 } \\
\hline A298E/C436H & $\begin{array}{l}\text { Increased peroxidase activity while } \\
\text { complete loss of oxygenase activity }\end{array}$ & $(212)$ \\
\hline \multicolumn{3}{|l|}{ P450cam } \\
\hline C357H & $\begin{array}{l}\text { Loss of oxygenase activity } \\
\text { Significant reduction in hydroxylation } \\
\text { activity }\end{array}$ & $(213,214)$ \\
\hline C357U & Decreased oxygenase activity & $(204)$ \\
\hline \multicolumn{3}{|l|}{ CPO } \\
\hline $\mathrm{C} 29 \mathrm{H}$ & $\begin{array}{l}\text { Chlorination, dismutation, peroxidation, } \\
\text { and epoxidation slightly decreased }\end{array}$ & $(215)$ \\
\hline
\end{tabular}


In the present study, I replaced proximal thiolate ligand in CPO with a His to mimic the ligation in classical peroxidases using site-directed mutagenesis. I utilized such spectroscopic techniques as proton NMR, CD spectroscopy, and UV-Visible spectrophotometry to investigate how the Cys $29 \rightarrow$ His mutation affects structural and catalytic properties of CPO to gain insights into the role of proximal Cys in CPO and other thiolate proteins.

\subsection{Experimental procedures}

\subsubsection{Materials}

A filamentous fungus, Aspergillus niger, was chosen for expression of the mutant CPO since expression in C. fumago produces WT CPO background that interferes with characterization of the mutant. The MGG029 strain A. niger was purchased from American Type Culture Collection. The plasmid coding for WT CPO (pCPO3.I-Amds) and cotransformation plasmid containing PyrG selection marker (pAB4-1) were purchased from TNO Microbiology and Systems Biology, Netherlands. The QuikChange II XL sitedirected mutagenesis kit and PfuUltra HF DNA polymerase were purchased from Stratagene (La Jolla, CA). Restriction enzymes were obtained from New England Biolabs (Ipswich, MA). Purified oligonucleotide primers were purchased from Eurofins MWG Operon (Huntsville, AL). De-ionized water for all experiments were prepared freshly by Milli-Q Biocel (Millipore, Billerica, MA). All other chemicals and materials were purchased from Fisher Scientific (Waltham, MA) unless otherwise stated.

\subsubsection{Construction of C29H CPO gene and plasmid propagation}

To probe the structural, spectroscopic, and functional role of proximal ligand in CPO, the C29H mutation was introduced into the pCPO3.I - AmdS (a PUC19-based 14-kb 
plasmid, Fig. 2.1) via polymerase chain reaction (PCR) amplification using the QuikChange II XL site-directed mutagenesis kit. The PCR reaction was carried out in Eppendorf thermal cycler (Mastercycler gradient) using PfuUltra HF DNA polymerase. The amounts of reagents used in a PCR reaction (total volume $=50 \mu \mathrm{L}$ ) are listed in Table 2.2. The following primers were used to introduce the $\mathrm{C} 29 \mathrm{H}$ mutation:

Coding strain - C29H primer 1

5’- CTACCGACTCTCGTGCTCCTCACCCAGCTCTGAACGCTCTTG - 3’

Non-coding strain - C29H primer 2

5’- CAAGAGCGTTCAGAGCTGGGTGAGGAGCACGAGAGTCGGTAG - 3’

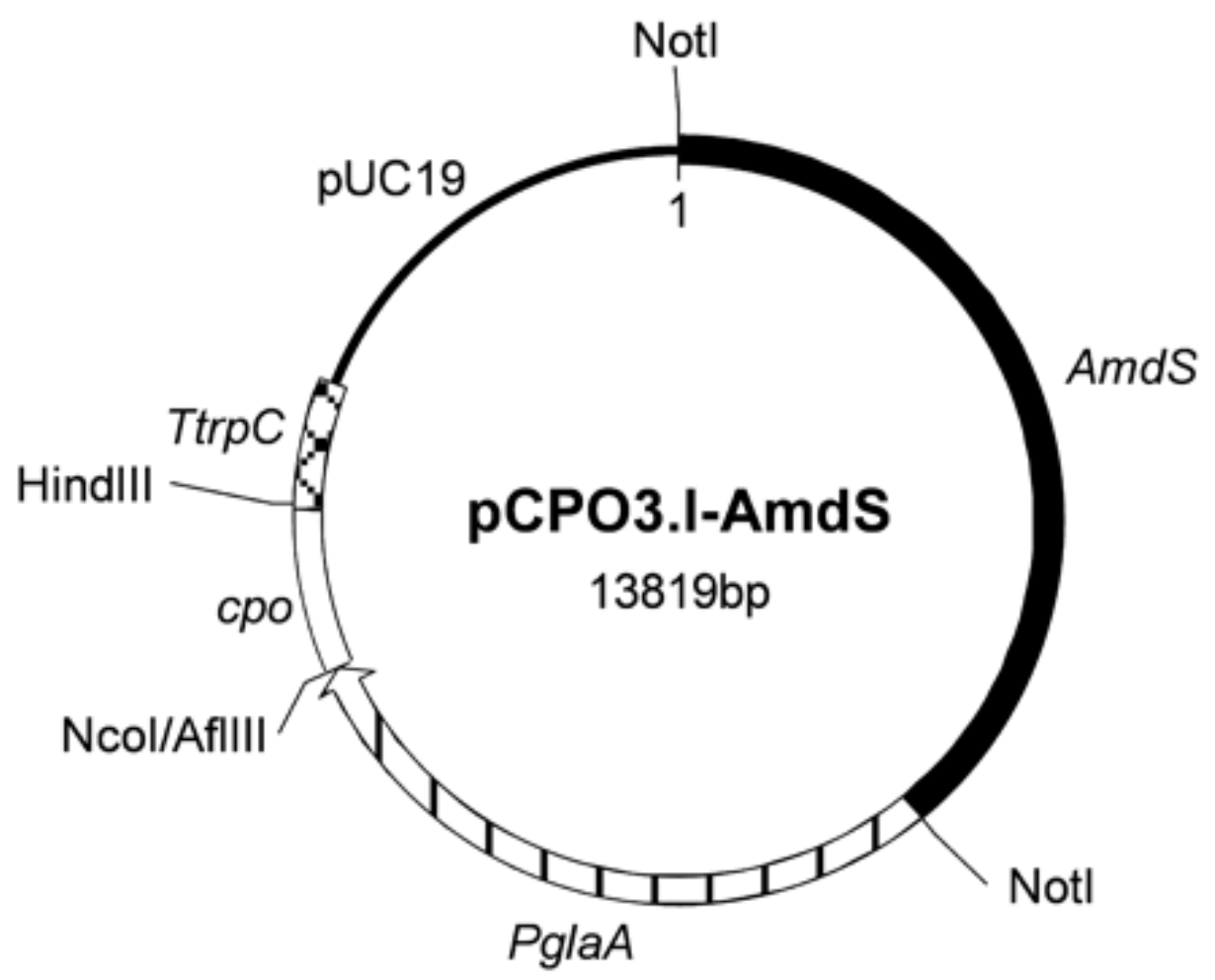

Figure 2.1. pCPO3.I-AmdS plasmid containing A. niger glucoamylase promoter (PglaA), CPO gene from C. fumago, A. nidulans anthranilate synthetase terminator (TtrpC), and $A$. nidulans acetamidase gene $(\mathrm{AmdS})$ ) as a selection marker allowing growth on acetamide as the sole source of nitrogen. 
Table 2.2. Reagents used in the PCR reaction.

\begin{tabular}{ll}
\hline Reagent & Amount \\
\hline 10Xreaction buffer & $5 \mu \mathrm{L}$ \\
\hline pCPO3.I - AmdS & $1 \mu \mathrm{L}$ \\
\hline Primer 1 & $125 \mathrm{ng}$ \\
\hline Primer 2 & $125 \mathrm{ng}$ \\
\hline dNTP mix & $1 \mu \mathrm{L}$ \\
\hline QuikSolution reagent & $3 \mu \mathrm{L}$ \\
\hline PfuUltra HF polymerase & $1 \mu \mathrm{L}$ \\
\hline DpnI & $1 \mu \mathrm{L}$ \\
\hline
\end{tabular}

The PCR was performed using the following steps:

1 cycle $\rightarrow 95^{\circ} \mathrm{C}$ for 1 minute

18 cycles $\rightarrow 95^{\circ} \mathrm{C}$ for 50 seconds (denaturation)

$60^{\circ} \mathrm{C}$ for 50 seconds (annealing)

$68^{\circ} \mathrm{C}$ for 14 minutes (extension)

1 cycle $\rightarrow 68^{\circ} \mathrm{C}$ for 7 minutes

The DNA product was digested with Dpn I restriction endonuclease to eliminate native plasmid. Mutated plasmid was propagated in E. Coli (DH5- $\alpha$ strain), and C29H mutation was confirmed by DNA sequencing (Fig. 2.2). Before transformation, both pCPO3.I and pAB4-1 (Fig. 2.3) were verified by DNA digestion and electrophoresis (Fig. 2.4).

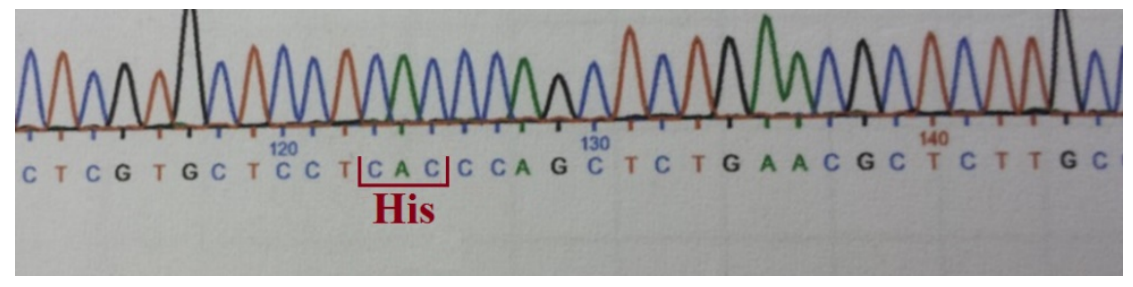

Figure 2.2. C29H pCPO sequencing results. 


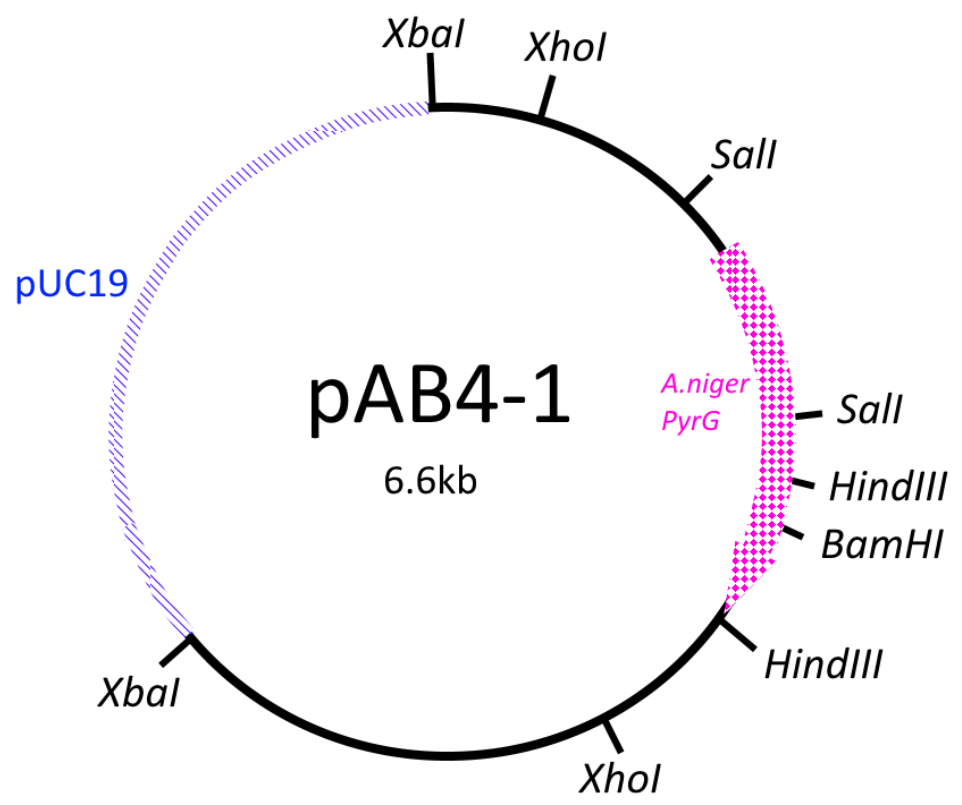

Figure 2.3. pAB4-1 plasmid containing A. niger PyrG gene as a selection marker allowing fungal growth without uridine supplementation.

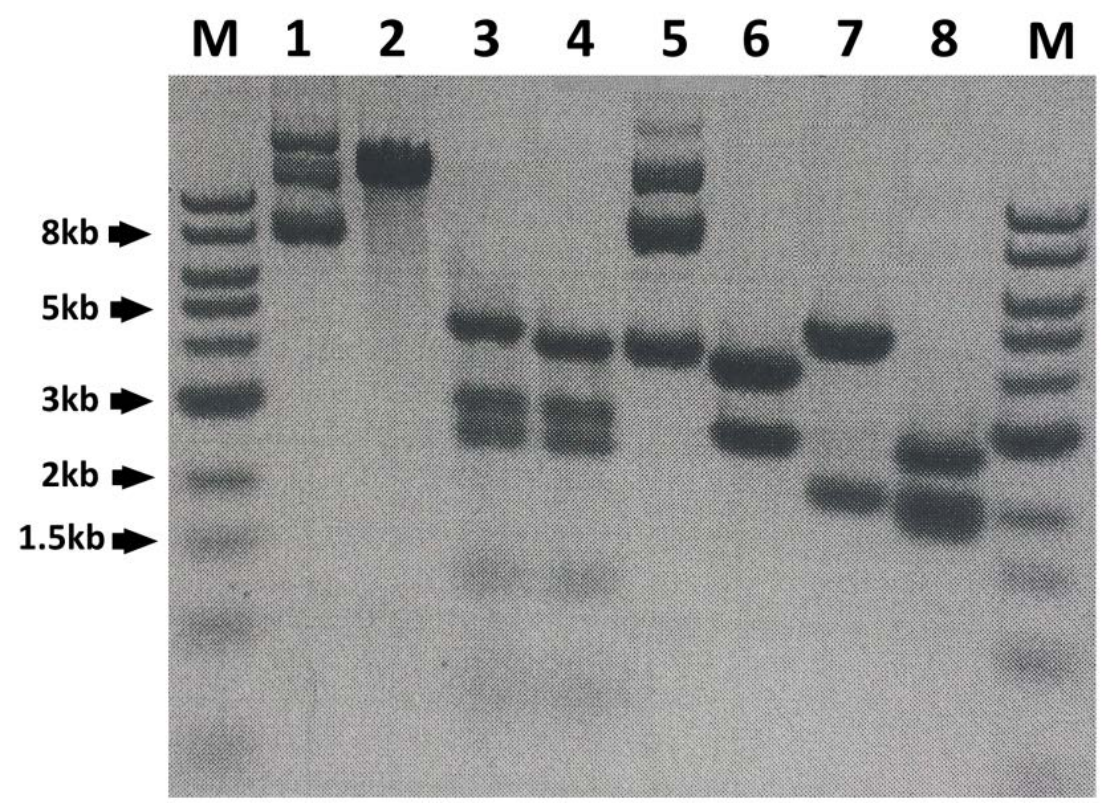

Figure 2.4. DNA electrophoresis. Restriction digestion of pCPO and pAB4-1. Lanes 1-4: pCPO3.I-AmdS, lanes 5-8 pAB4-1, lanes 1 and 5- undigested DNA, lane 2 - HindIII, lane 3 - AflIII, lane 4 - Hind III and AflIII, lane 6 - XbaI, lane 7 - BamHI, lane 8 - XbaI and BamHI. 


\subsubsection{Transformation of Aspergillus niger with C29H pCPO and mutant selection}

The pCPO3.I-C29H was co-transformed with pAB4-1 into the protease deficient MGG029 strain of A. niger (prtT gla::fleor pyrG) using procedures described previously $(97,216)$ with the following modifications. Brown conidiospores were inoculated in 50$\mathrm{mL}$ minimal medium (MM) containing 0.5\% yeast extract, 0.5\% Casamino acids, 5\% maltose, 1\% glucose, 70mM NaNO 3 , $7 \mathrm{mM} \mathrm{KCl,} 6 \mathrm{mM} \mathrm{KH}_{2} \mathrm{PO}_{4}, 6 \mathrm{mM} \mathrm{K}_{2} \mathrm{HPO}_{4}, 2 \mathrm{mM}$ $\mathrm{MgSO}_{4}, 10 \mathrm{mM}$ uridine, $25 \mu \mathrm{L}$ of carbenicillin (100 mg/mL stock), 50uL of the Hutner trace elements solution (217). The mini-culture was incubated at $250 \mathrm{rpm}$ and $37^{\circ} \mathrm{C}$ for 1 day and then at $30^{\circ} \mathrm{C}$ until white-yellow mycelium is observed (2-5 days). After disruption of the mycelium with a homogenizer, the culture was transferred to 1-L MM and incubated at $30^{\circ} \mathrm{C}$ and $250 \mathrm{rpm}$ for $16-20$ hours followed by protoplast preparation and DNA

transformation using polyethylene glycol as described previously (216). Successful transformants were selected on polyacrylamide plates lacking uridine supplementation and used to express the mutant protein.

Each clone from the acrylamide plates was inoculated in 50-ml MM lacking uridine and yeast extract, and incubated at $250 \mathrm{rpm}$ and $22^{\circ} \mathrm{C}$ for 1 week. The ABTS assay, described below, was used to determine the clone with the highest protein yield, which was used to set up large scale protein expression.

\subsubsection{Expression and purification of C29H CPO}

The A. niger spores (1 plate per $1 \mathrm{~L}$ medium) were inoculated into 48-L MM lacking uridine and yeast extract and containing 10\% fructose, carbenicillin (50 mg/L), and 100 $\mu \mathrm{M} \delta$-aminolevulinic acid. Cultures were incubated at $250 \mathrm{rpm}$ and $25^{\circ} \mathrm{C}$ for 3 days and then at $22^{\circ} \mathrm{C}$ for 4 more days. Then, the medium was filtered through glass fiber filter 
circles G6 and concentrated down to $50 \mathrm{~mL}$ in the Amicon stirring pressure cells. Concentrated sample was dialyzed against 25 mM phosphate buffer, pH 5.9 and filtered through $0.45 \mu \mathrm{m}$ membrane.

Mutant CPO was purified using AKTA prime chromatography system. All buffers used during purification were filtered through $0.45 \mu \mathrm{m}$ membrane and de-gassed for 15 minutes. The protein sample $(50 \mathrm{~mL})$ in $25 \mathrm{mM}$ phosphate buffer, $\mathrm{pH} 5.9$ was filtered through $0.45 \mu \mathrm{m}$ membrane and applied onto a $100-\mathrm{mL}$ column packed with $50 \mathrm{~mL}$ of diethylaminoethanol (DEAE) sepharose resin, and washed with $25 \mathrm{mM}$ phosphate buffer, pH 5.9 while gradually increasing $\mathrm{NaCl}$ concentration. The $\mathrm{C} 29 \mathrm{H}$ CPO eluted at approximately 30\% $0.5 \mathrm{M} \mathrm{NaCl}$. Fractions with Reinheitzahl (RZ) values $\left(\mathrm{A}_{420} / \mathrm{A}_{280}\right)$ above 0.7 were combined and concentrated down to about $1 \mathrm{~mL}$ using Millipore 30,000 centrifugal filter unit. The sample was filtered through $0.45 \mu \mathrm{m}$ membrane and applied onto a gel filtration column containing $500 \mathrm{~mL}$ of Sephadex G75 resin and eluted with 25 mM phosphate buffer, pH 5.9 (Fig. 2.5).

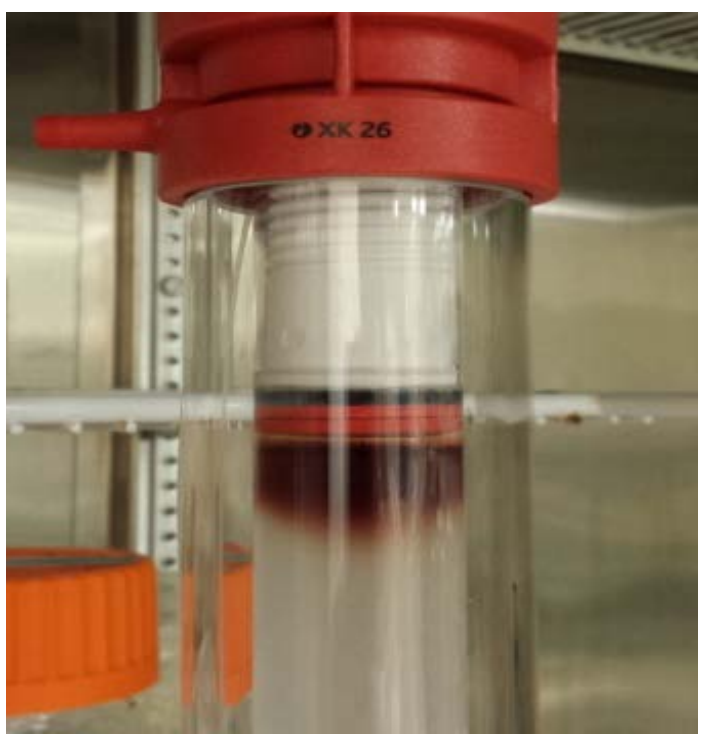

Figure 2.5. C29H CPO sample loaded on a gel filtration column. 
Fractions with RZ values between 1.2 - 1.5 were used in all subsequent studies. Purity of the mutant CPO was assessed by sodium dodecyl sulfate polyacrylamide gel electrophoresis (SDS-PAGE) (Fig. 2.6).

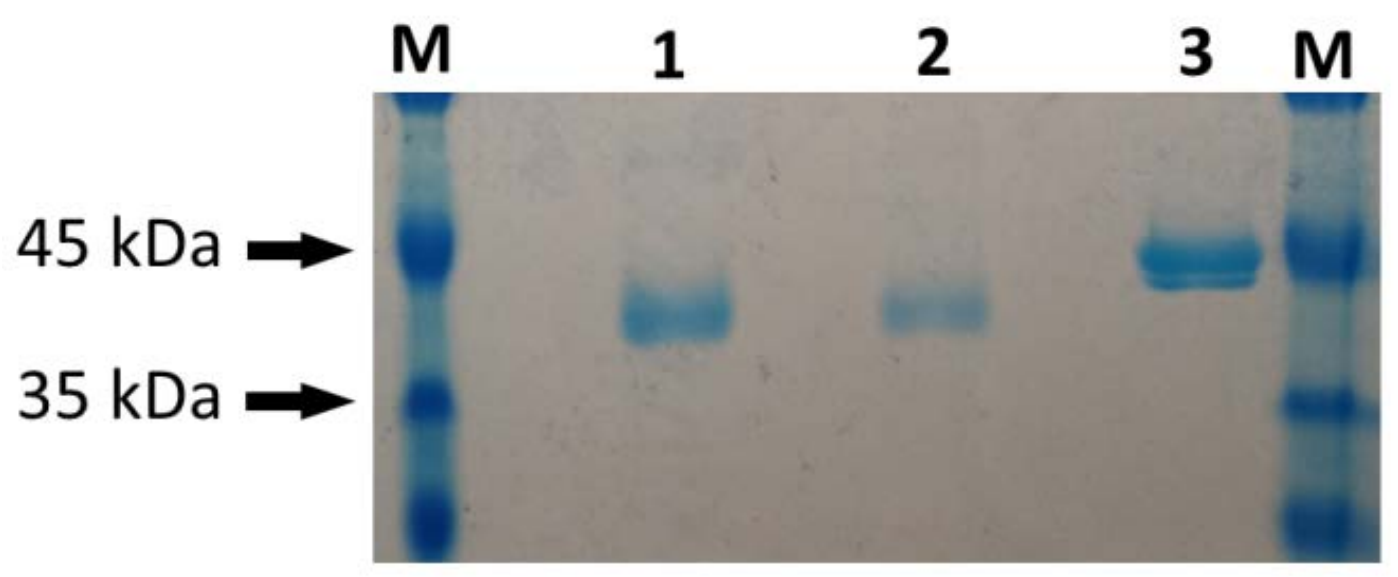

Figure 2.6. SDS-PAGE. WT and C29H CPO. Lanes 1 and 2 - C29H, lane 3 - WT CPO.

\subsubsection{Spectroscopic characterization of C29H CPO}

Structural characterization of $\mathrm{C} 29 \mathrm{H} \mathrm{CPO}$ was performed using $600 \mathrm{MHz}$ nuclear magnetic resonance spectrometer, JASCO CD spectrometer, and VARIAN UV-Vis spectrophotometer (Cary 300 Bio).

\subsubsection{Proton nuclear magnetic resonance spectroscopy}

Proton nuclear magnetic resonance (NMR) spectroscopy was used to confirm C29H mutation of CPO. A $600-\mathrm{MHz}$ NMR spectrometer was used to investigate the NMR spectral properties of the ferric low-spin cyanide complexes of C29H CPO in comparison with cyanide complexes of HRP and WT CPO. HRP was chosen for comparison since it is a classical peroxidase with the imidazole proximal ligation. The spectra of the enzyme- $\mathrm{CN}^{-}$ complexes in deuterated phosphate buffer were taken at room temperature. I chose to work with dCPO-CN complexes since the high-spin nature of WT dCPO produces broad spectral 
resonances providing limited information about the structure of the protein. When bound to cyanide, heme iron in WT CPO converts to a low spin, and protein-CN complex spectrum can provide more information about structural properties of the active site than the native ferric form of the protein.

The spectra of a $0.8 \mathrm{mM}$ cyanide-bound C29H CPO (RZ=1.2), 1.0 mM WT CPO, and 1.0 mM HRP were collected in a 5-mm NMR tubes. Protein samples were exchanged with deuterated $25 \mathrm{mM}$ potassium phosphate buffer before analysis using Centricon from Amicon. Protein-cyanide complexes were prepared by addition of an excess amount of freshly prepared $1 \mathrm{M} \mathrm{KCN}$ in deuterated phosphate buffer, $\mathrm{pH}$ 5.9. The concentration of the C29H sample was estimated from the theoretical extinction coefficient of $44,015 \mathrm{M}^{-1} \mathrm{~cm}^{-1}$ at $280 \mathrm{~nm}$ as calculated by ProtParam tool (expasy.org). For WT CPO, the signals in the downfield region represent protons from amino acid residues in the proximal and distal sites of CPO and heme substituents while upfield shifts belong to beta-protons of amino acid residues near the center and the heme propionate and vinyl groups (183).

\subsubsection{Circular dichroism spectroscopy}

The CD spectra of $1 \mu \mathrm{M} \mathrm{C29H}$ and WT CPO in $5 \mathrm{mM}$ sodium acetate buffer, pH 5.5 were collected in the 190-260 $\mathrm{nm}$ range at room temperature using 5-mm quartz cuvettes. Three spectra were averaged for each protein. The secondary structures were determined using CDSSTR method and reference \#4 (218). CDSSTR is a program that performs calculations of the secondary structures using a particular number of proteins in the reference set. Those proteins that do not reflect characteristics of the test protein are removed from the basis set, and secondary structures are assigned by the singular value decomposition algorithm (DICHROWEB). 


\subsubsection{UV-Visible spectrophotometry}

The UV-Vis absorption spectra of C29H and WT CPO in phosphate buffer, pH 5.9 were collected in the $250-700 \mathrm{~nm}$ range at room temperature using 1-cm quartz cuvettes. Three spectra were averaged for each protein. To study the effect of $\mathrm{pH}$, the spectra of C29H and WT CPO in $100 \mathrm{mM}$ phosphate-citrate buffer or $100 \mathrm{mM}$ phosphate buffer (pH 2.5 - 10.0) were collected using 1-cm quartz cuvettes at room temperature. Buffer exchange was performed using PD-10 column from GE Healthcare Life Sciences.

\subsubsection{Ligand binding study}

Cyanide $\left(\mathrm{CN}^{-}\right)$and carbon monoxide (CO) are known substrates for WT CPO (219, 220). The absorption spectra of C29H and WT CPO in $25 \mathrm{mM}$ phosphate buffer, pH 5.9 in the absence and presence of substrates were collected using 1-cm quartz cuvettes. The spectra were recorded in $250-700 \mathrm{~nm}$ range at room temperature using UV-Vis spectrophotometer. The final concentration of cyanide in the sample was $100 \mathrm{mM}$. In $\mathrm{CN}^{-}$binding study, the spectra were collected upon initial addition of the substrate and after 15-minute incubation of the sample at room temperature. For CO binding, WT and C29H CPO were reduced with sodium dithionite and purged with $\mathrm{CO}$ for 1 minute. To eliminate

the possibility of the proximal His to Cys switch, I also prepared C29H/C79H and C29H/C79H/C87H CPO as described in sections 2.2.2-2.2.4 and performed CO binding study with those two mutants as well. The primers used to construct $\mathrm{C} 29 \mathrm{H} / \mathrm{C} 79 \mathrm{H}$ and C29H/C79H/C87H CPO are given in Fig. A1.

\subsubsection{Enzymatic activity assays}

All reactions were initiated by the addition of hydrogen peroxide (except for catalase assay) and monitored for 180 seconds with a UV/Vis spectrophotometer at room 
temperature. The initial rates of reactions were calculated from the linear portions of the reaction curves.

\subsubsection{Peroxidation assay (ABTS)}

Peroxidase activity of CPO was measured by the oxidation of ABTS that is commonly used as a substrate to estimate peroxidation activity of heme enzymes (221). The reaction system consisted of either WT or mutant CPO in 100 mM phosphate-citrate buffer (pH 2.75 - 6.0) containing $36.8 \mu \mathrm{M}$ ABTS. After $\mathrm{H}_{2} \mathrm{O}_{2}(3.6 \mathrm{mM})$ addition, the reaction was monitored for the formation of oxidized ABTS at $405 \mathrm{~nm}$. Protein amounts used for the assay at various $\mathrm{pH}$ are listed in Table 2.3. The activity of CPO was decreased at higher $\mathrm{pH}$; therefore, larger amounts of protein were used to detect peroxidation.

Table 2.3. Protein amounts used in ABTS assay at different $\mathrm{pH}$.

\begin{tabular}{lll}
\hline $\mathbf{p H}$ & $\mathbf{W T}(\boldsymbol{\mu M})$ & $\mathbf{C 2 9 H}(\boldsymbol{\mu M})$ \\
\hline 2.75 & 0.003 & 0.03 \\
\hline $3.0,3.25,3.5$ & 0.03 & 0.03 \\
\hline 4.0 & 0.1 & 0.03 \\
\hline 4.5 & 0.5 & 0.1 \\
\hline $5.0,5.5$ & 1.0 & 0.1 \\
\hline 6.0 & 2.0 & 0.1 \\
\hline
\end{tabular}

\subsubsection{Chlorination assay (MCD)}

The MCD assay was used to determine the chlorination activity of CPO that catalyzes conversion of MCD into DCD (222). The reaction system consisted of either WT or C29H CPO in 100 mM phosphate-citrate buffer (pH 2.75, 3.0, 3.5, 4.0, 4.5, 5.0) containing 20 $\mathrm{mM} \mathrm{KCl}$ and $0.17 \mathrm{mM} \mathrm{MCD}$. After $\mathrm{H}_{2} \mathrm{O}_{2}(2.2 \mathrm{mM})$ addition, the reaction was monitored at $278 \mathrm{~nm}$, at which MCD absorbs. In the pH range 2.75-4.0, $0.005 \mu \mathrm{M}$ WT CPO was used 
in the assay while $0.05 \mu \mathrm{M}$ was used at $\mathrm{pH} 4.5$ and 5.0. As for C29H CPO, $0.6 \mu \mathrm{M}$ mutant protein was used in MCD reactions at all $\mathrm{pH}$.

\subsubsection{Catalatic assay}

Catalatic activity was measured by monitoring decomposition of hydrogen peroxide (34). The reaction system consisted of $0.05 \%$ hydrogen peroxide in either $100 \mathrm{mM}$ phosphate buffer, $\mathrm{pH} 2.4$ or $100 \mathrm{mM}$ phosphate-citrate buffer (pH 3.0, 3.5, 4.0, 4.5, 5.0, 5.5, 6.0). After either $0.5 \mu \mathrm{M} \mathrm{WT}$ or $0.5 \mu \mathrm{M}$ C29H CPO was added, the reaction was monitored at $240 \mathrm{~nm}$.

\subsubsection{Epoxidation assay (styrene)}

Styrene epoxidation assay (15) was performed in $100 \mathrm{mM}$ phosphate-citrate buffer (pH 2.75, 3.0, 3.5, 4.0, 4.5, 6.0, 7.5) containing $0.3 \mathrm{mM}$ styrene and either $0.3 \mu \mathrm{M}$ WT or $\mathrm{C} 29 \mathrm{H}$ CPO. After addition of $2.2 \mathrm{mM} \mathrm{H}_{2} \mathrm{O}_{2}$, the reaction was monitored at $262 \mathrm{~nm}$, at which styrene absorbs.

\subsubsection{Calculations of specific activities}

Specific activities (SA) were calculated using equation 2.1 while the change in the amount of a substrate or product over time was calculated using equation 2.2, where $\mathrm{A}$ is absorbance, $\varepsilon$ is the extinction coefficient at a specific wavelength, $b$ is the length of a pathway, and c is the concentration of the substrate or product.

$$
\begin{gathered}
S A=\frac{\mu m o l \text { substrate or product } / \mathrm{sec}}{\mu \mathrm{mol} \text { enzyme }} \\
A=\varepsilon b c,
\end{gathered}
$$

The extinction coefficients used to calculate substrate or product concentration change are 
listed in Table 2.4.

Table 2.4. Extinction coefficients of oxidized ABTS, MCD, hydrogen peroxide, and styrene.

\begin{tabular}{lll}
\hline Substrate or product & Wavelength & $\begin{array}{l}\text { Extinction coefficient } \\
\left(\mathbf{m M}^{-\mathbf{1}} \mathbf{c m}^{-\mathbf{1}} \mathbf{)}\right.\end{array}$ \\
\hline Oxidized ABTS & $405 \mathrm{~nm}$ & 36.8 \\
\hline $\mathrm{MCD}$ & $278 \mathrm{~nm}$ & 12.2 \\
\hline $\mathrm{H}_{2} \mathrm{O}_{2}$ & $240 \mathrm{~nm}$ & 0.0436 \\
\hline Styrene & $262 \mathrm{~nm}$ & 0.6225 \\
\hline
\end{tabular}

The styrene extinction coefficient at $262 \mathrm{~nm}$ was determined to be $0.6225 \pm 0.0781 \mathrm{mM}^{-}$ ${ }^{1} \mathrm{~cm}^{-1}$ from the slope of a plot of absorbance versus concentration (from 3 trials) (Fig. 2.7). Styrene was dissolved in $100 \mathrm{mM}$ phosphate-citrate buffer, $\mathrm{pH}$ 3.25. Since styrene absorbance decreases with time because of its poor solubility in aqueous solutions, all solutions with styrene were freshly prepared for each trial.

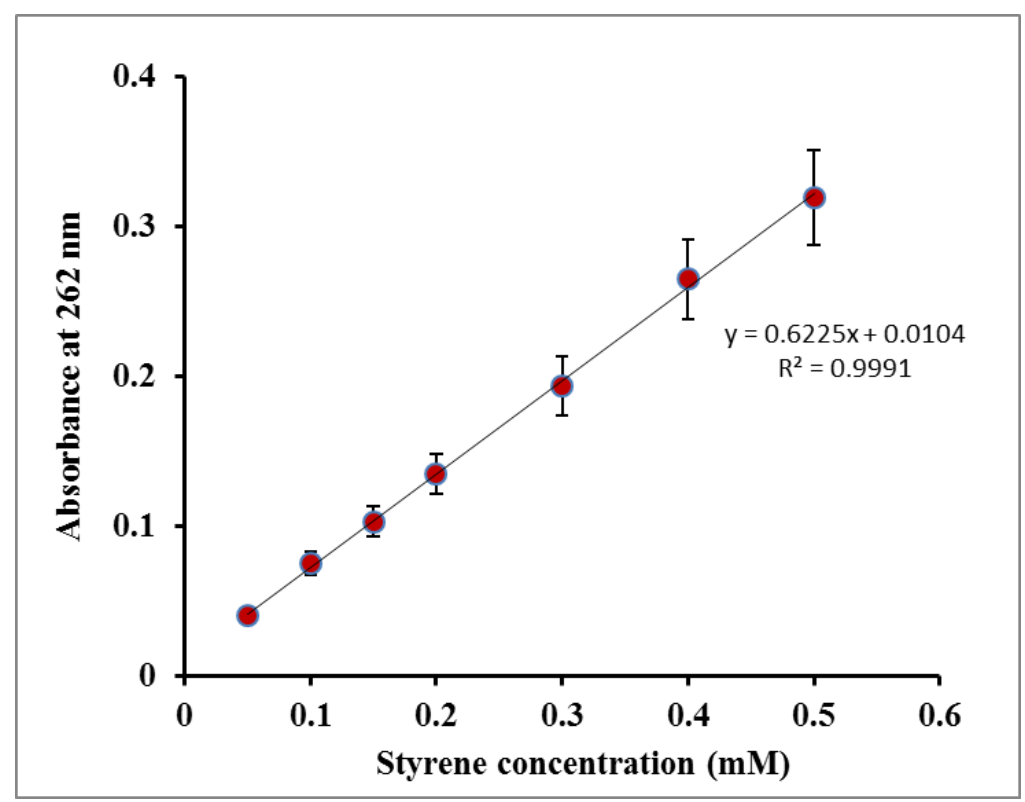

Figure 2.7. A plot of styrene concentration versus absorbance at $262 \mathrm{~nm}$ (average of 3 trials). Error bars represent standard deviation.

\subsection{Results and discussion}

\subsubsection{The proton NMR study}


The chemical shifts of two beta protons of Cys 29 in the WT were observed at +39.5 ppm and -21.5 ppm, which are consistent with results reported by Wang et al. (183). The signal of His $\mathrm{H} \varepsilon$ of $\mathrm{HRP}$ is located at $-31.0 \mathrm{ppm}$, consistent with results reported previously (223). The chemical shifts at $+60.0 \mathrm{ppm}$ and $-28.5 \mathrm{ppm}$ were assigned to His $29 \mathrm{H} \delta$ and $\mathrm{H} \varepsilon$ of the mutant enzyme, respectively. The difference in the location of $\mathrm{H} \delta$ signals in HRP and C29H might be attributed to the differences in the orientation of the proximal His with respect to the heme. Thus, the substitution of the proximal Cys in $\mathrm{C} 29 \mathrm{H} \mathrm{CPO}$ was confirmed by ${ }^{1} \mathrm{H}$-NMR spectroscopy.

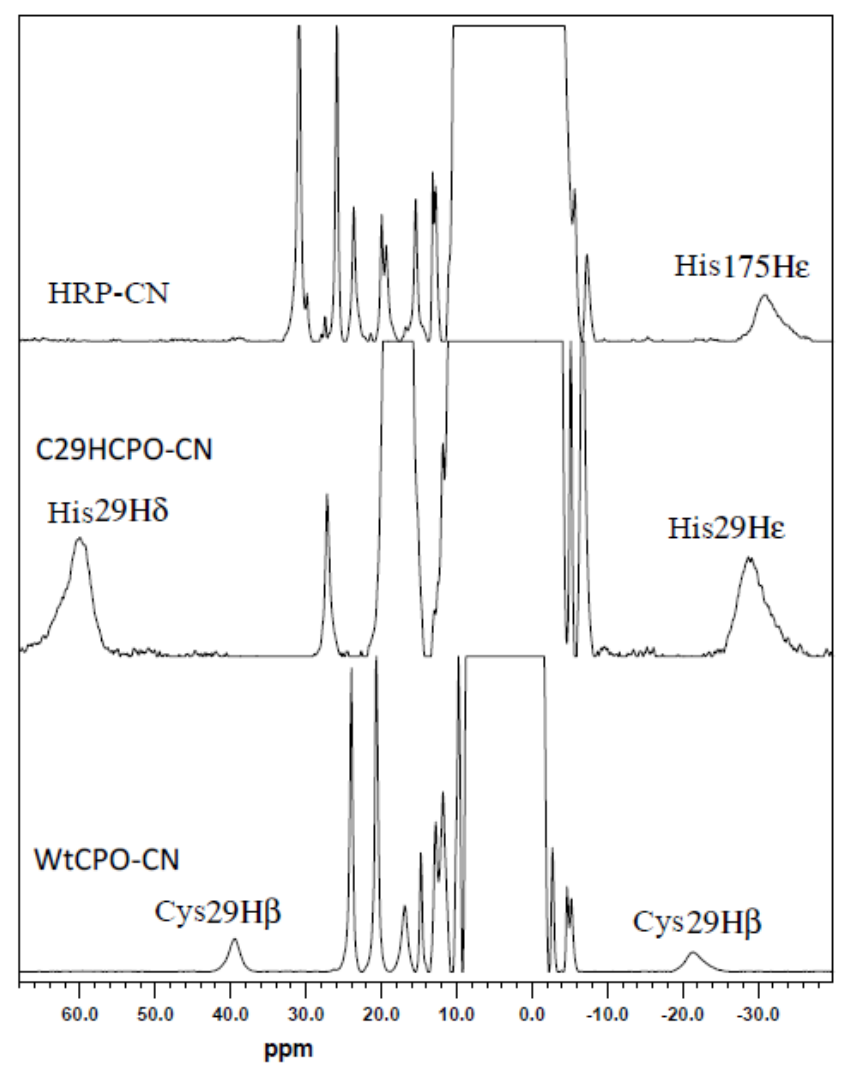

Figure 2.8. 600-MHz proton NMR spectra of cyanide complexes of HRP, WT and C29H $\mathrm{CPO}$ in deuterated phosphate buffer, $\mathrm{pH}$ 5.9.

\subsubsection{Circular dichroism of WT and variant CPO}


Circular dichroism spectroscopy was used to determine if substitution of Cys 29 with a His had a significant effect on the secondary structure of $\mathrm{CPO}$, which in turn might affect the substrate affinity and enzymatic activity of the enzyme. Since the van der Waals volume of a histidine $\left(118 \mathrm{~A}^{3}\right)$ is larger than that of a cysteine $\left(86 \mathrm{~A}^{3}\right)(224,225)$, it is likely that some perturbation of the mutant active site occurred which may affect the protein secondary structure, possibly in the proximal helix region where the proximal ligand is located. This, in turn, could affect the redox potential of the heme iron and biochemical properties of the protein. Thus, cysteine ligation might be important for maintaining a particular architecture of the active site necessary for catalytic diversity.

The secondary structures of the C29H and WT CPO were estimated using JASCO CD spectrometer and CDSSTR (Fig. 2.9). For WT, alpha-helical and beta-sheet contents were found to be $30 \%$ and $25 \%$, respectively. For C29H, alpha-helices and beta-sheets were estimated at $34 \%$ and $20 \%$, respectively. The differences in the secondary structural elements of the WT and the mutant are quite small and are unlikely to contribute significantly to the functional differences of these two enzymes. However, possible changes in the tertiary structure of the protein, especially proximal helix orientation (226), could affect the redox potential and reactivity of C29H CPO.

Using CD spectroscopy, it is possible to determine if tertiary structural changes occur upon mutation; however, this method is not specific enough to indicate the region where the changes occur. In order to determine the locations and magnitude of the tertiary structural changes, it would be necessary to solve crystal structure of the mutant and compare it with that of the WT. 


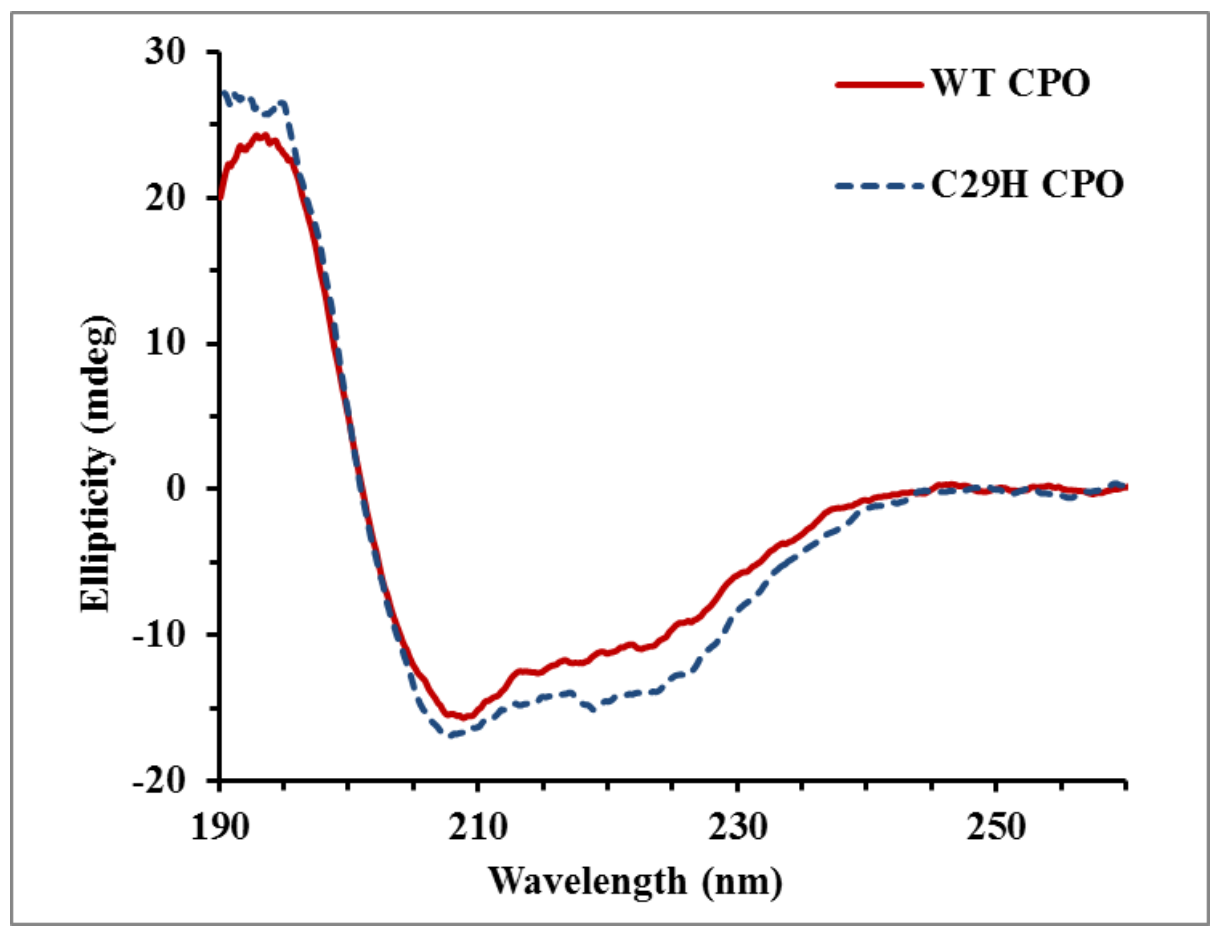

Figure 2.9. CD spectra of $1.0 \mu \mathrm{M}$ WT and C29H CPO in acetate buffer, pH 5.5.

\subsubsection{UV-Visible spectroscopic properties of WT and C29H CPO}

$\mathrm{UV}$-Vis spectrophotometry was used to obtain a spectroscopic signature of CPO that can provide useful information about the electronic, magnetic, and molecular structure of the heme active center. The spectrum of WT CPO shows the Soret peak at $398 \mathrm{~nm}$ as well as $\beta, \alpha$, and charge transfer (CT) bands at 515, 545, and $650 \mathrm{~nm}$, respectively (Fig. 2.10 and Table 2.5). The peaks at 515 and $650 \mathrm{~nm}$ are considered the high-spin markers indicating that ferric WT CPO is in high-spin pentacoordinate form. The Soret band of $\mathrm{C} 29 \mathrm{H}$ is red shifted to $419 \mathrm{~nm}$. The Q bands shifted as well to 540 and $570 \mathrm{~nm}$ indicating that ferric $\mathrm{C} 29 \mathrm{H}$ possesses low-spin heme that is coordinated to a sixth ligand, possibly water or an amino acid in the distal active site. Furthermore, it has been proposed that the relative intensities of the $\alpha$ and $\beta$ bands can be an indication of how close in energy the $a_{2 u}$ and $\mathrm{a}_{1 \mathrm{u}}$ orbitals of porphyrin are (179, Figs. 1.20 and 1.21). 


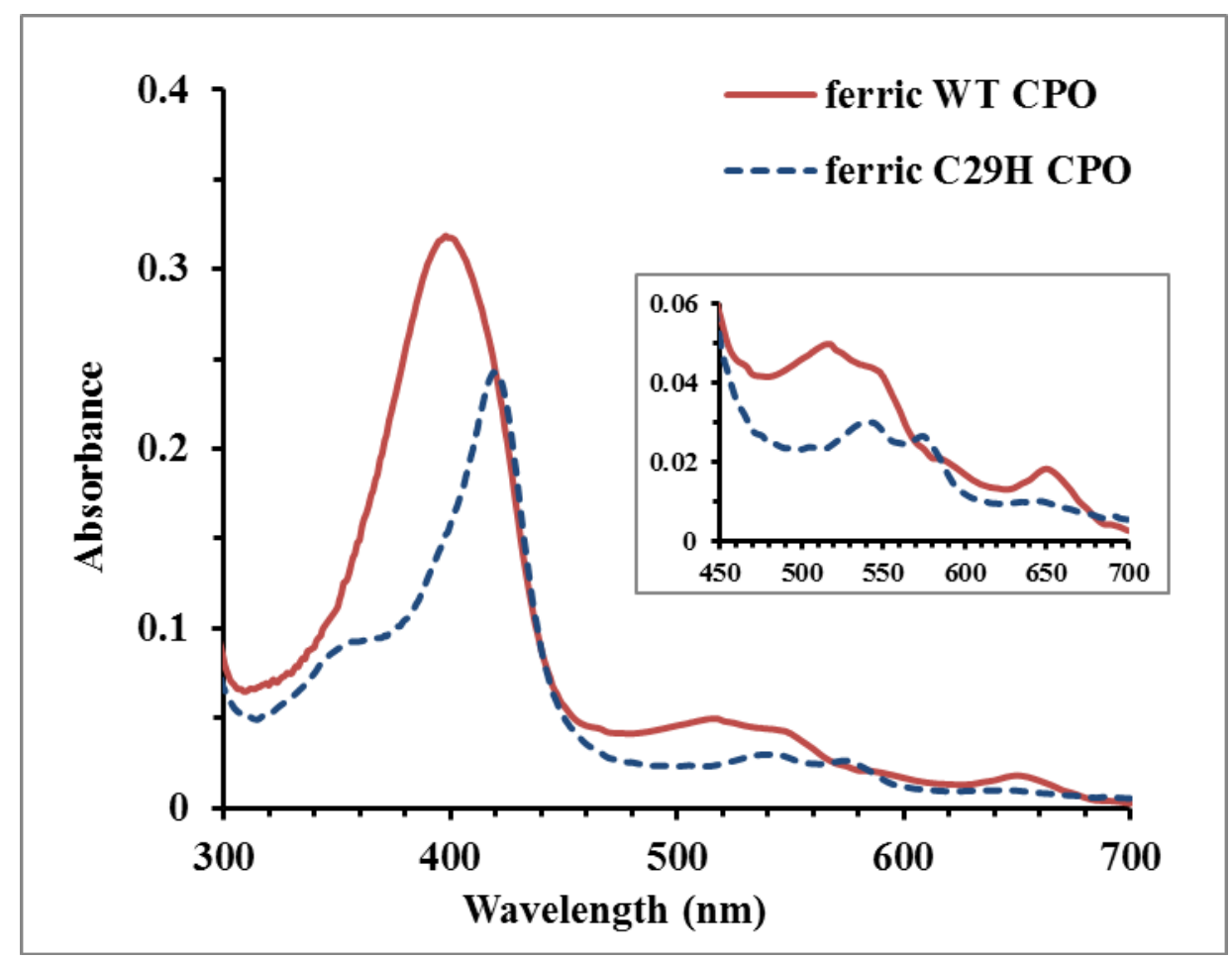

Figure 2.10. UV-Vis spectra of WT and C29H CPO in $25 \mathrm{mM}$ phosphate buffer, pH 5.9.

Table 2.5. UV-Vis spectral properties of ferric WT and C29H CPO in phosphate buffer, pH 5.9 (n.s.-no shoulder, s.s.-slight shoulder, l.i.-low intensity).

\begin{tabular}{lccccc}
\hline Protein & $\begin{array}{c}\boldsymbol{\delta} \text { band } \\
(\mathbf{n m})\end{array}$ & $\begin{array}{c}\text { Soret } \\
(\mathbf{n m})\end{array}$ & $\begin{array}{c}\boldsymbol{\beta} \text { band } \\
(\mathbf{n m})\end{array}$ & $\begin{array}{c}\boldsymbol{\alpha} \text { band } \\
(\mathbf{n m})\end{array}$ & $\begin{array}{c}\text { CT } \\
(\mathbf{n m})\end{array}$ \\
\hline \multirow{2}{*}{ WT CPO } & -- & 398 (n.s.) & 515 & 550 & 650 \\
\hline C29H & 354 & 419 (s.s.) & 540 & 570 & 645 (l.i.) \\
\hline
\end{tabular}

If the two orbitals are very similar in energy, the $\alpha / \beta$ intensity ratio will be small. The $\alpha / \beta$ intensity ratios of WT and C29H CPO suggest that $\mathrm{a}_{2 \mathrm{u}}$ and $\mathrm{a}_{1 \mathrm{u}}$ porphyrin orbitals in WT are closer in energy than those in $\mathrm{C} 29 \mathrm{H}$ mutant. In addition, the spectrum of $\mathrm{C} 29 \mathrm{H}$ exhibits a clear $\delta$-band, which indicates that the heme iron is in a low spin. Moreover, the $\delta$-band was proposed to be a result of an electron cloud density around the heme, and hence can be viewed as a sensor for the heme redox state (227). 
To investigate the $\mathrm{pH}$ influence on the structure of $\mathrm{CPO}$, the absorption spectra of both WT and C29H CPO in a phosphate buffer at different pH were obtained. It is well known that WT CPO transforms from the active high-spin state to the inactive low-spin form at alkaline $\mathrm{pH}$ (228). The pKa for the transition of the active WT CPO to the inactive C420 form was determined to be 7.5 by Blanke et al. (228). It has been proposed that at this $\mathrm{pH}$, deprotonation of an amino acid in the active site results in further ionization of other residues, which in turn induces a conformational change in the protein. Later, it has been shown that this ionizable amino acid interacts with the heme iron. It is possible that these interactions prevent ligand binding to the alkaline form since exogenous molecules were shown to be unable to bind heme (228). Therefore, the inactivation of CPO might be a result of its inability to bind $\mathrm{H}_{2} \mathrm{O}_{2}$, which is required for formation of the catalytic species, Cpd I.

Wild-type $\mathrm{CPO}$ at neutral and acidic $\mathrm{pH}$ exists as a pentacoordinate high-spin form as suggested by its UV-Visible spectrum (Fig. 2.11). At pH 8.0 and above, the heme iron state changes to a low-spin as evident from the red shift of the Soret band and decrease in absorbance at 515 and $650 \mathrm{~nm}$ (high spin markers). My results are consistent with previously reported data and show that transition to the alkaline low-spin, hexacoordinate form occurs between $\mathrm{pH} 7$ and 8 (Fig. 2.11).

Different results are observed for $\mathrm{C} 29 \mathrm{H}$, which stays in a hexacoordinate, low-spin state over a wide range of $\mathrm{pH}$ from very acidic to alkaline (Figs. 2.12 and 2.13). The spectrum starts changing at $\mathrm{pH}$ 3.5. The intensity of the Soret band starts decreasing and a new peak at around $400 \mathrm{~nm}$ is observed at $\mathrm{pH} 2.5$ indicating transitioning from the low spin species to the high spin form. 


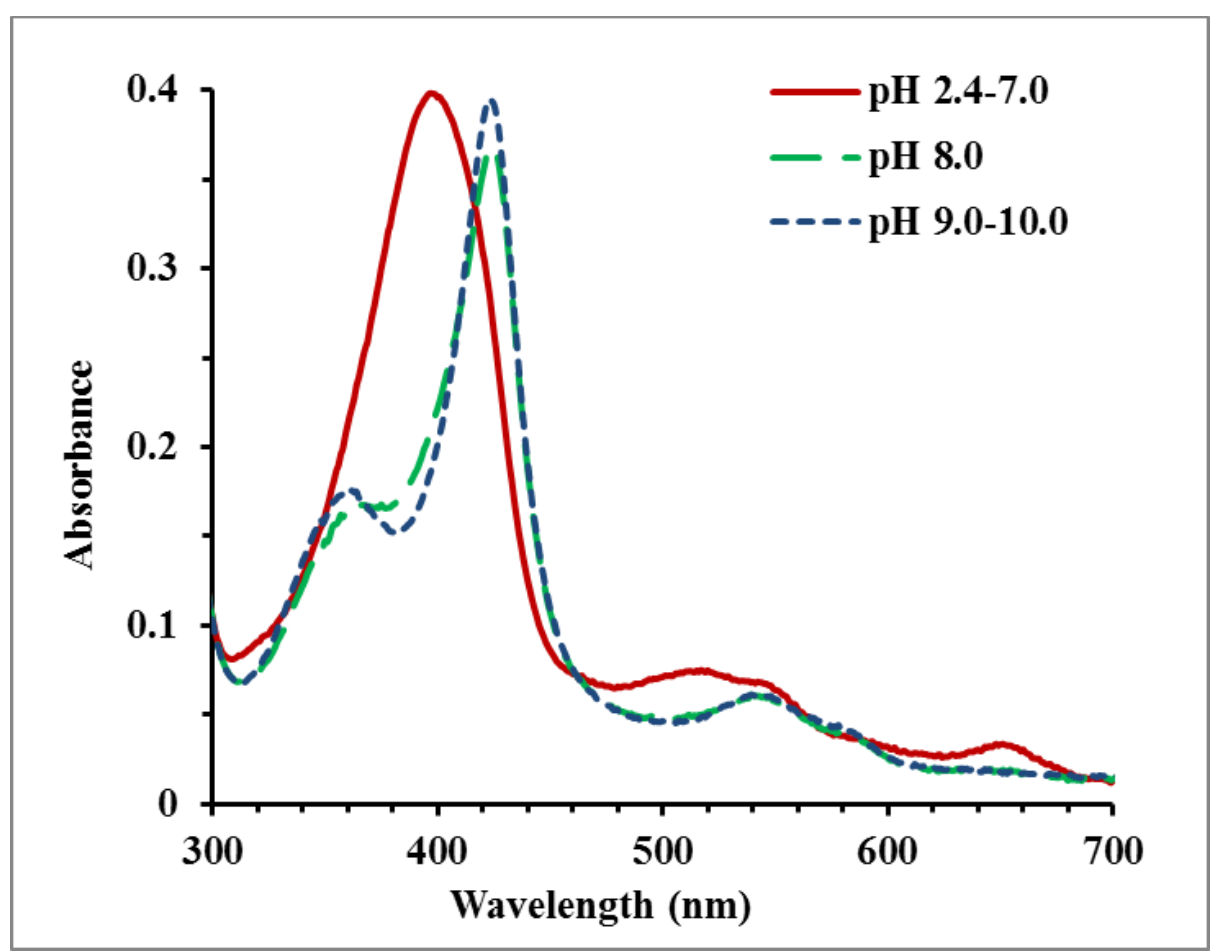

Figure 2.11. UV-Vis absorption spectra of WT CPO in phosphate or phosphate-citrate buffer at different $\mathrm{pH}$.

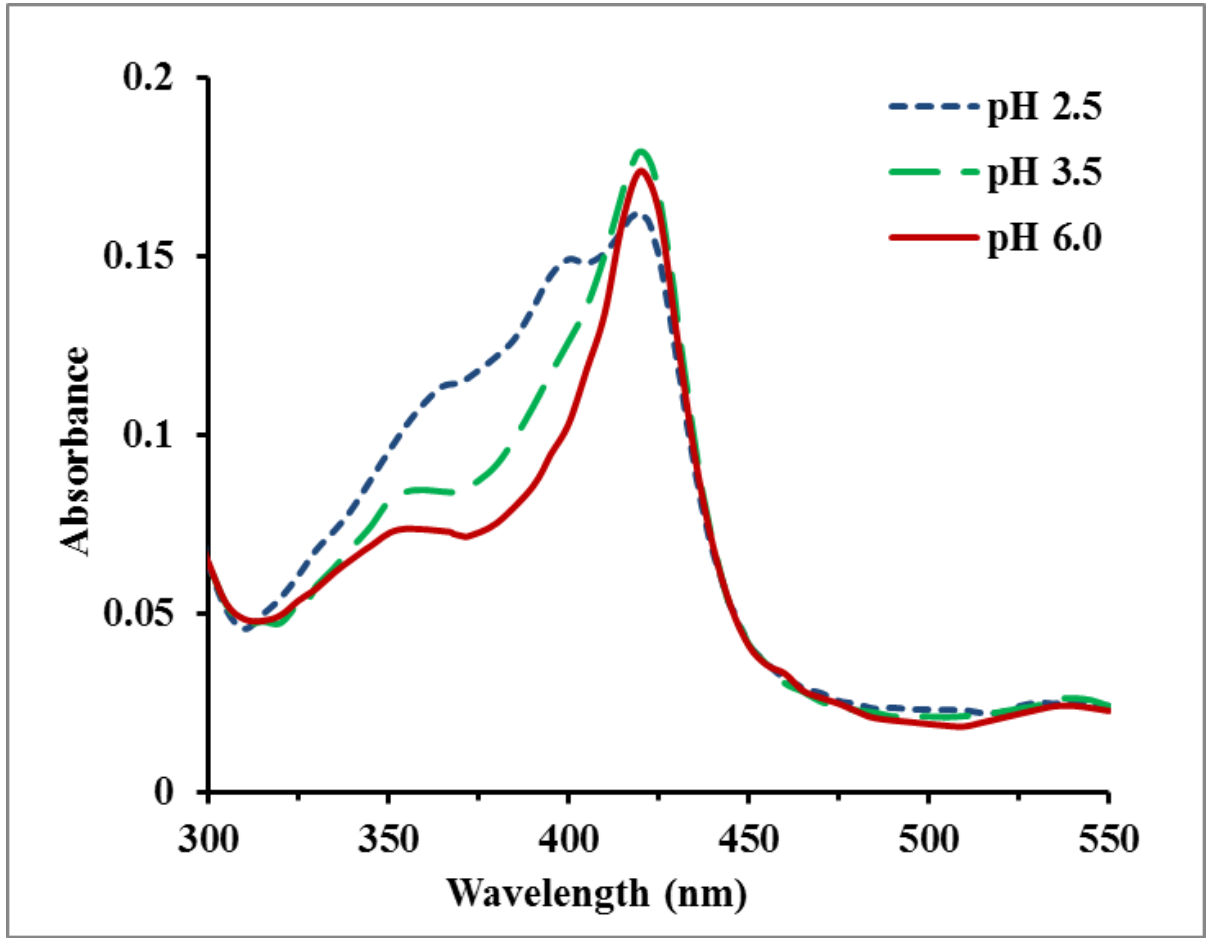

Figure 2.12. UV-Vis absorption spectra of C29H CPO in phosphate or phosphate-citrate buffer in the acidic $\mathrm{pH}$ range. 


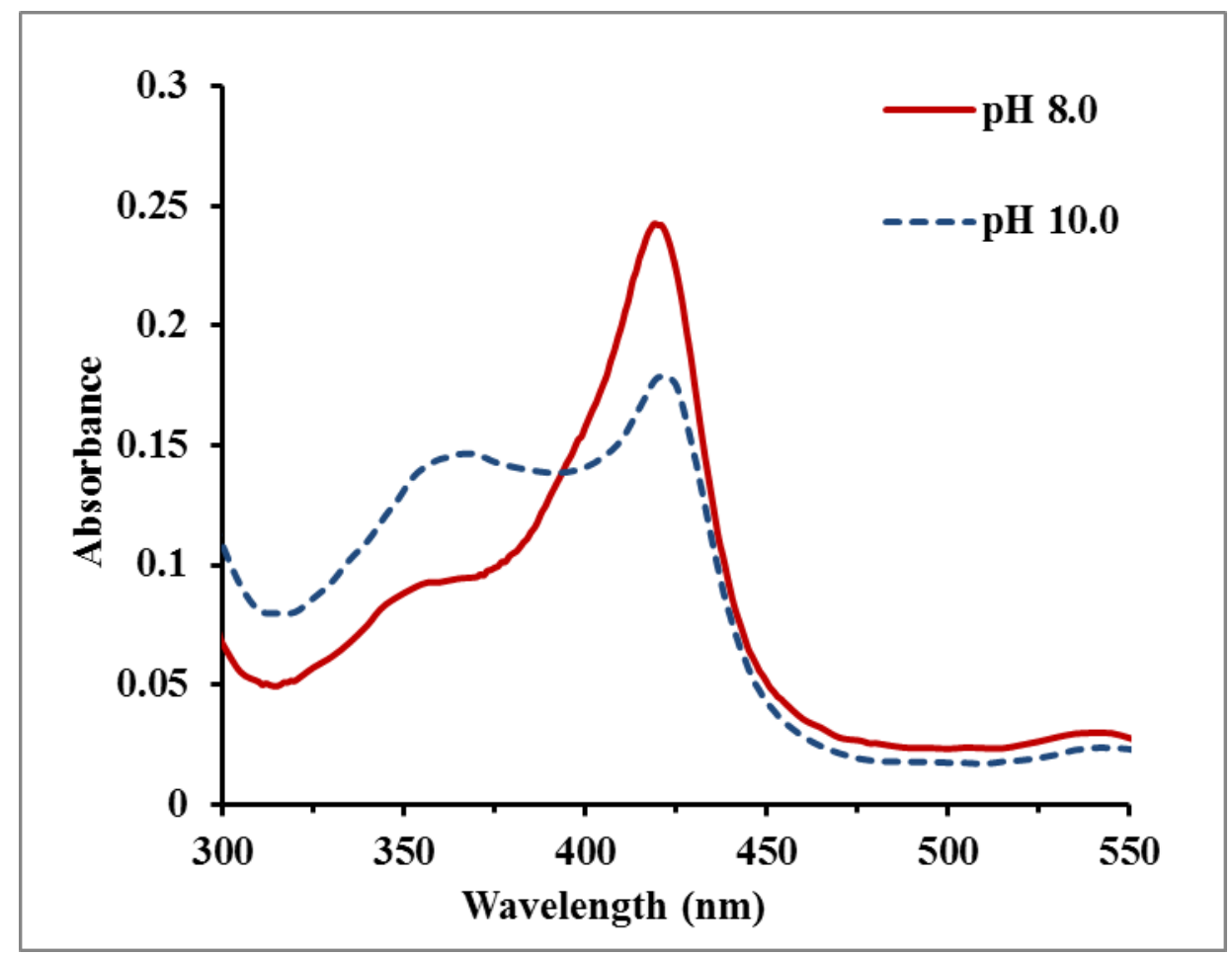

Figure 2.13. UV-Vis absorption spectra of C29H CPO in phosphate-citrate buffer in basic $\mathrm{pH}$.

These spectroscopic results also suggest that the distal ligand, possibly water or an amino acid, is very acidic with a low pKa. Even though it would be unusual for water to have such a low pKa (below 3.5), it is possible that its interactions with heme result in a pKa decrease.

\subsubsection{CO binding to WT, C29H CPO, C29H/C79H, and C29H/C79H/C87H CPO}

Binding of ligands to CPO depends on the size of the ligands and their pKa values. Quite a few ligands were found to bind either to ferric or ferrous CPO. The ligands include but are not limited to dioxygen, carbon monoxide, cyanide, pyridine, nitrate, nitrite, thiocyanate, imidazole, organic isocyanides, and acetate $(174,219)$. In general, the binding properties of CPO are similar to those of classical peroxidases. An exception is the binding of carbon monoxide to ferrous CPO to produce an absorption peak at around $450 \mathrm{~nm}$, 
similar to cytochromes P450. It has been postulated that thiolate ligation in P450s and CPO enzymes is responsible for the observed unique 450-nm absorption. (9). Several studies utilized heme Soret band absorption location for a ferrous protein-CO complex to investigate the nature of the proximal ligand, stating that cysteine and histidine, upon binding CO, would produce the peak near $450 \mathrm{~nm}$ and $420 \mathrm{~nm}$, respectively $(106,196$, 211-215).

My results with $\mathrm{C} 29 \mathrm{H}$ CPO mutant shown in Figure 2.14 indicate that proximal cysteine is not a prerequisite for the P450-like spectroscopic signature. The Soret band of $\mathrm{C} 29 \mathrm{H}$ upon reduction with sodium dithionite shifts from $419 \mathrm{~nm}$ to $407 \mathrm{~nm}$. The spectrum of C29H-CO complex (444 nm) is almost identical to that of the WT-CO (445 nm) although the two enzymes have dramatically different proximal ligation (Fig. 2.15).

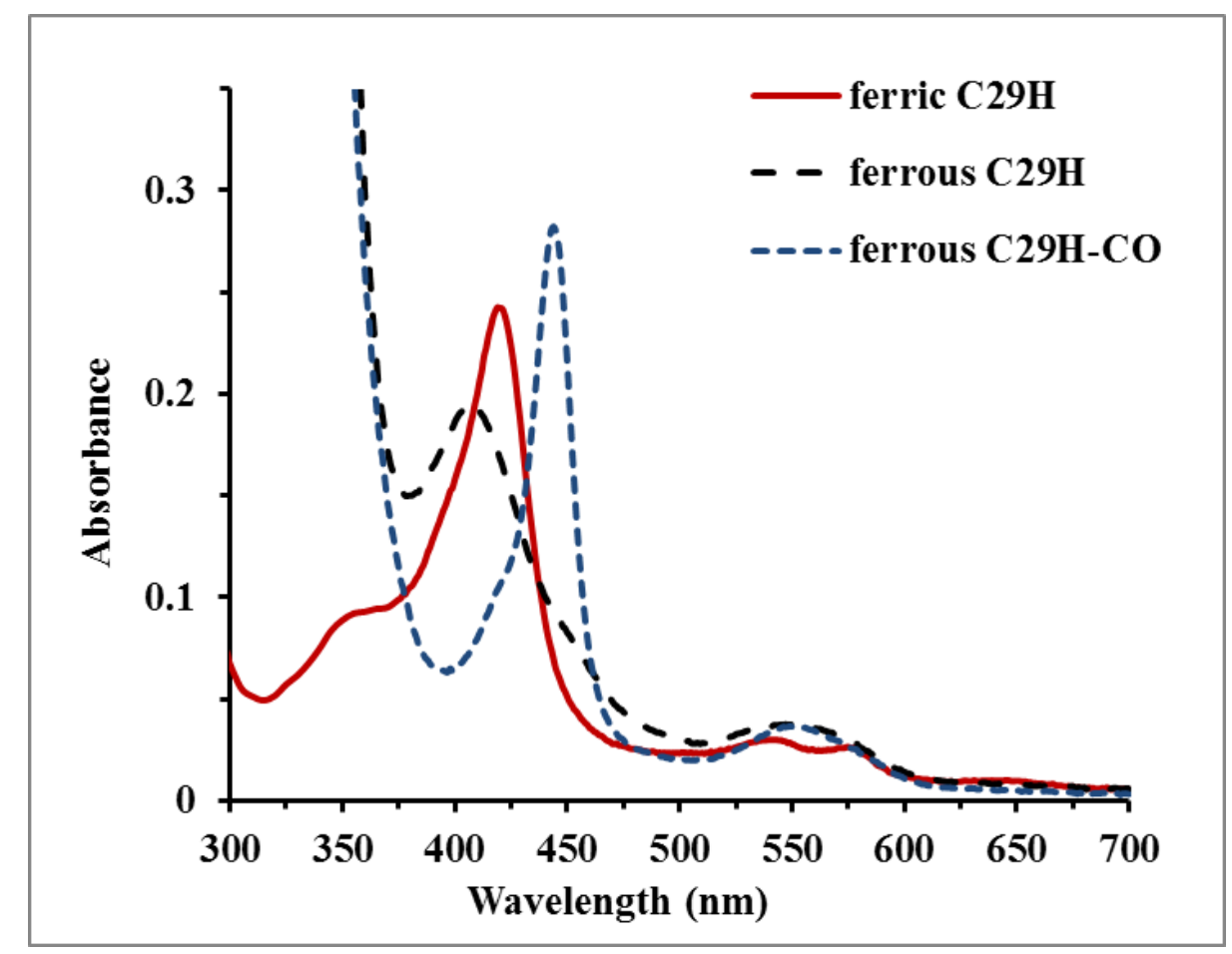

Figure 2.14. UV-Vis absorption spectra of ferric C29H, ferrous C29H, and ferrous C29HCO complex in phosphate buffer, pH 5.9. 


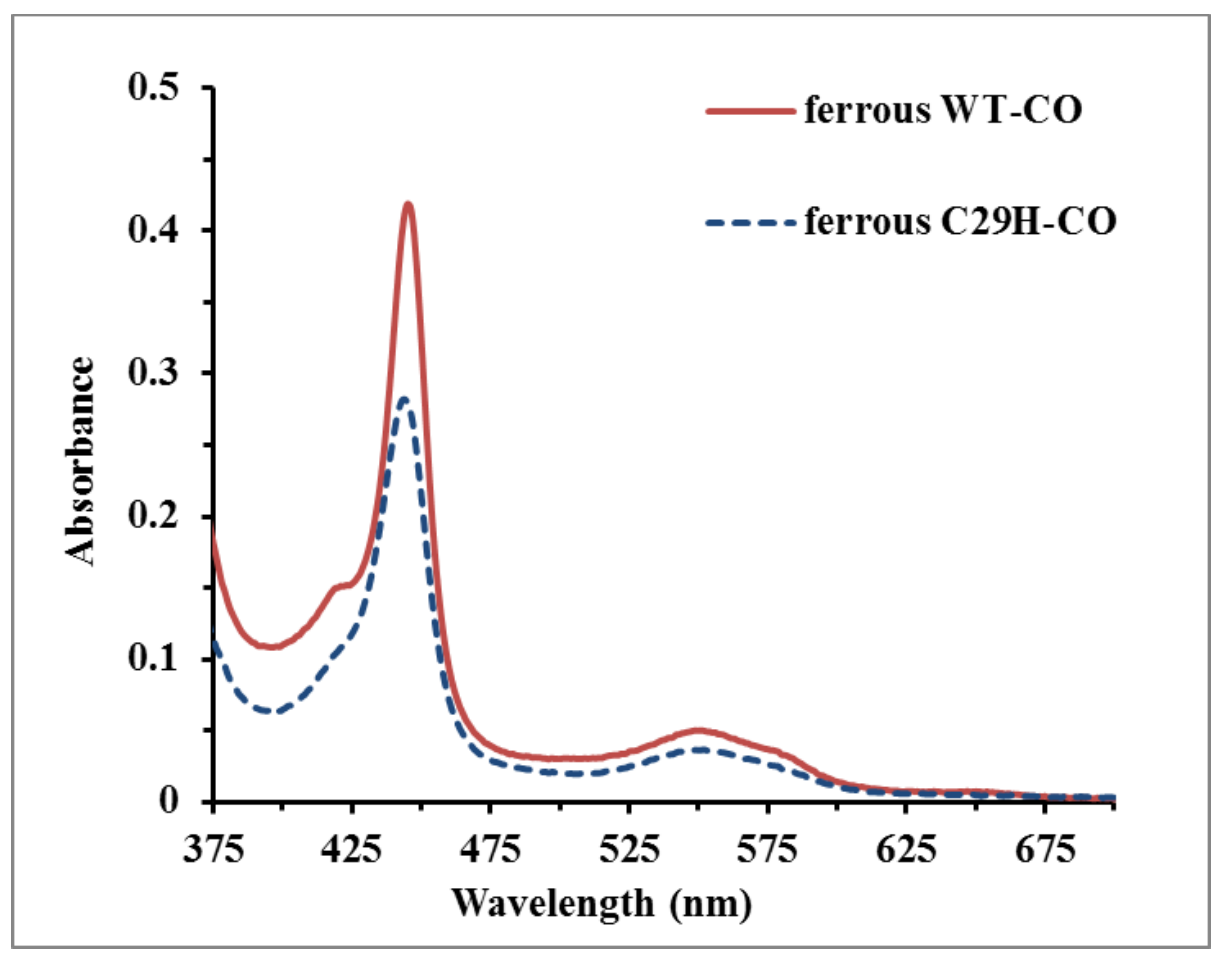

Figure 2.15. UV-Vis absorption spectra of ferrous WT-CO and C29H-CO at pH 5.9.

It can be argued that the absorption peak near $450 \mathrm{~nm}$ might be the result of the switch of a His to a Cys ligation since CPO possesses two more cysteines (Fig 2.16). However, even though such a switch is possible, it is unlikely because of the location of both Cys residues in the helix $\mathrm{C}$ and their involvement in the formation of a disulfide bond. Swapping of a proximal His in $\mathrm{C} 29 \mathrm{H} \mathrm{CPO}$ would require significant changes in the tertiary structure of the protein and is very unlikely to occur.

To further investigate the possibility of the His to Cys switch, Cys 79 and Cys 87 were also replaced with a His to create double and triple CPO mutants, C29H/C79H and C29H/C79H/C87H. The spectra of mutant-CO complexes are shown in Figure 2.17. Both mutant-CO absorb at $444 \mathrm{~nm}$, similarly to the WT-CO. Since C29H/C79H/C87H mutant is devoid of Cys, it cannot form a thiolate ligation. This finding supports my hypothesis that thiolate is not a prerequisite for the P450-like spectroscopic signature of CPO. 


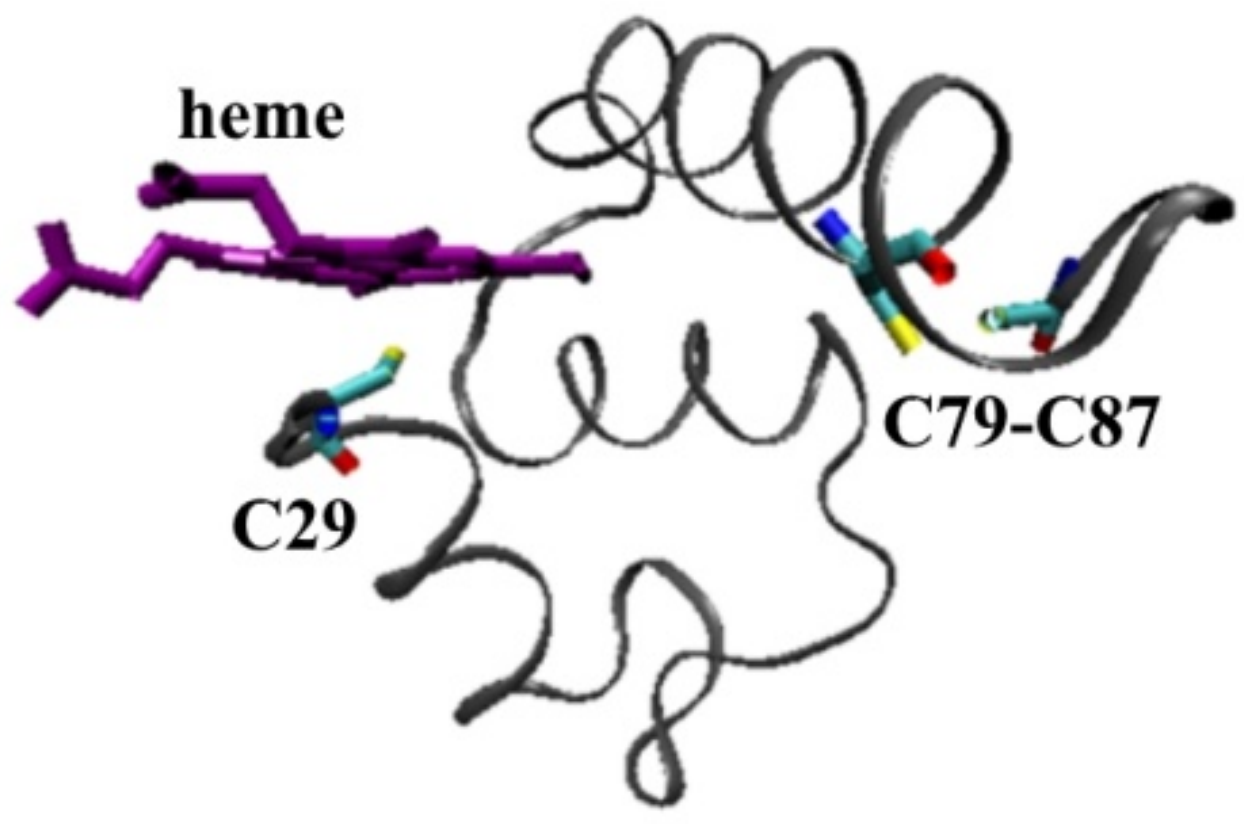

Figure 2.16. Locations of 3 cysteine residues in CPO. The following pdb file was used to visualize CPO: $1 \mathrm{CPO}(75)$.

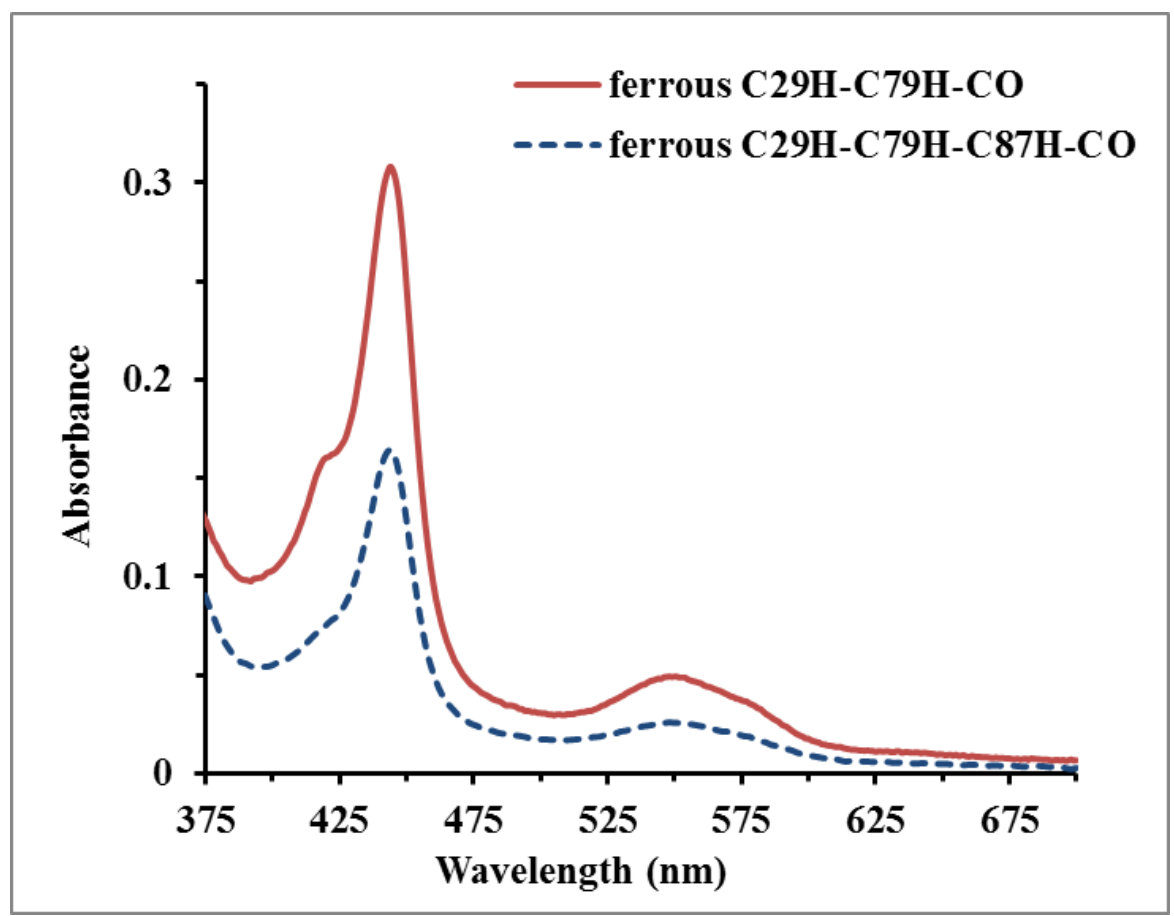

Figure 2.17. UV-Vis absorption spectra of ferrous C29H/C79H-CO and $\mathrm{C} 29 \mathrm{H} / \mathrm{C} 79 \mathrm{H} / \mathrm{C} 87 \mathrm{H}-\mathrm{CO}$ at $\mathrm{pH}$ 5.9. 


\subsection{5 $\mathrm{CN}^{-}$binding to WT and $\mathrm{C29H}$ CPO}

Cyanide is a known inhibitor of CPO and binds to other heme proteins as well. The spectra of the WT CPO and its cyanide complex were obtained at pH 5.9. Since cyanide is a very weak acid with a pKa of 9.14 (229), it binds to the ferric state of CPO in its protonated form at $\mathrm{pH}$ 5.9. Upon binding cyanide, the spin state of CPO changes from high to low as evident from the Soret band shift from $398 \mathrm{~nm}$ to $439 \mathrm{~nm}$, the presence of the $\delta$ band, and the disappearance of the CT band at $650 \mathrm{~nm}$ (Fig. 2.18, Table 2.6). After 15-min incubation, the spectrum of the WT-CN complex remained the same.

The change in the spin state of iron occurs because of the rearrangement of electrons in the molecular orbital of heme. According to the ligand-field theory originally developed by Bethe (230) and later extended by others (231, 232), ligand electrons interact with electrons in d orbitals of the iron (Fig. 1.21) resulting in a particular energy gap between the $d(x y), d(x z), d(y z)$ and $d\left(x^{2}-y^{2}\right), d\left(z^{2}\right)$ orbitals called the crystal-field splitting energy. If this energy difference is larger than the electron pairing energy, then electrons fill up the lower energy orbitals $\left(d_{x y}, d_{x z}, d_{y z}\right)$ first resulting in a low spin state. If the crystal-field splitting energy is smaller than the electron pairing energy, then electrons fill up empty $\mathrm{d}_{\mathrm{x} 2-}$ y2 and $\mathrm{d}_{\mathrm{z} 2}$ orbitals before pairing in $\mathrm{d}_{\mathrm{xy}}, \mathrm{d}_{\mathrm{xz}}$, and $\mathrm{d}_{\mathrm{yz}}$ orbitals, resulting in a high spin state. Since cyanide is a strong field ligand, its binding to iron leads to a high crystal-field splitting energy in d orbitals resulting in a low spin heme.

Slightly different behavior is observed for the C29H-CN complex, the Soret peak of which shifts with time from $436 \mathrm{~nm}$ to $424 \mathrm{~nm}$, in contrast to the unchanged WT-CN Soret absorption (Fig. 2.19). This indicates that cyanide competes with another axial ligand, possibly water, and has a lower affinity for the mutant than for the WT. 


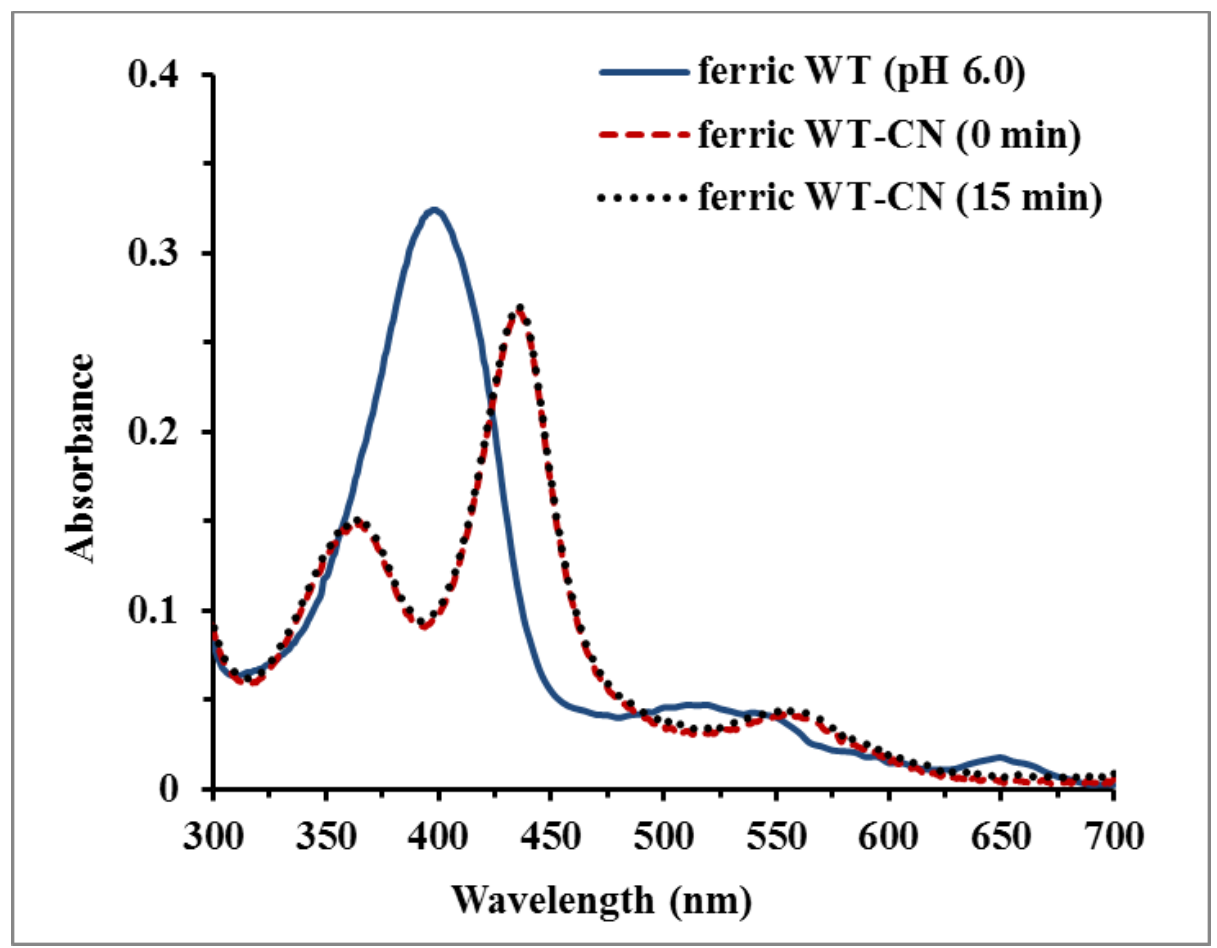

Figure 2.18. UV-Vis spectra of ferric WT and WT-CN in phosphate buffer, pH 5.9.

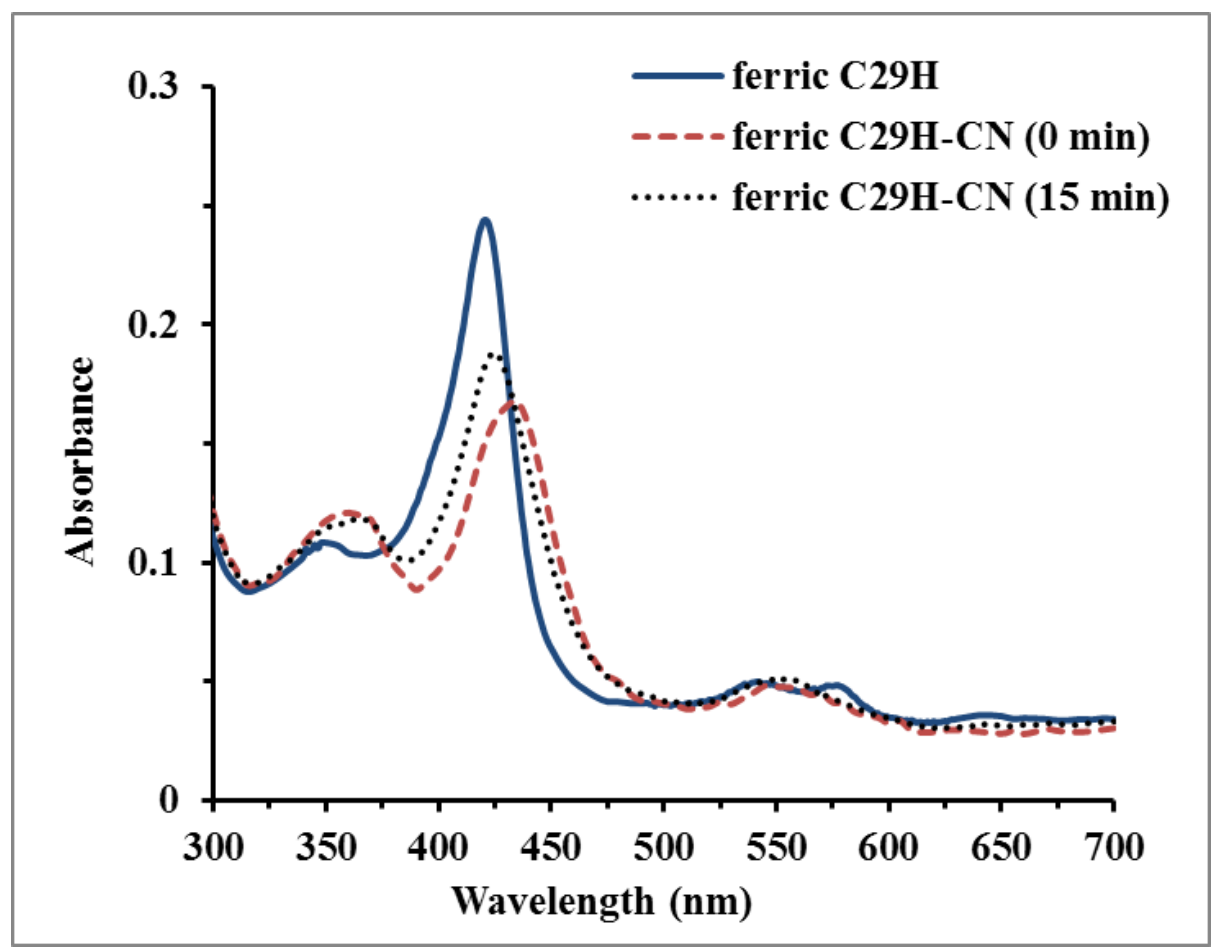

Figure 2.19. UV-Vis spectra of ferric C29H and C29H-CN in phosphate buffer, pH 5.9. 
Table 2.6. UV-Vis spectral properties of ferric WT and C29H CPO and their cyanide complexes at $\mathrm{pH}$ 5.9.

\begin{tabular}{lccccc}
\hline Protein & $\begin{array}{c}\boldsymbol{\delta} \text { band } \\
(\mathbf{n m})\end{array}$ & $\begin{array}{c}\text { Soret } \\
(\mathbf{n m})\end{array}$ & $\begin{array}{c}\boldsymbol{\beta} \text { band } \\
(\mathbf{n m})\end{array}$ & $\begin{array}{c}\boldsymbol{\alpha} \text { band } \\
(\mathbf{n m})\end{array}$ & $\begin{array}{c}\mathbf{C T} \\
(\mathbf{n m})\end{array}$ \\
\hline WT CPO & -- & 398 & 515 & 550 & 650 \\
\hline WT-CN & 364 & 439 & -- & 564 & -- \\
\hline C29H CPO & 354 & 419 & 540 & 570 & 645 \\
\hline C29H-CN & 364 & $436 \rightarrow 424$ & -- & 558 & - \\
\hline
\end{tabular}

\subsubsection{Catalytic properties of WT and C29H CPO}

To investigate the effect of proximal ligand substitution on the catalytic capabilities of $\mathrm{CPO}$, the activities of $\mathrm{C} 29 \mathrm{H}$ and WT were measured in 4 different assays: chlorination of MCD, dismutation of $\mathrm{H}_{2} \mathrm{O}_{2}$, epoxidation of styrene, and peroxidation of ABTS (Figs. 2.20-2.23). C29H CPO showed a 10 -fold and more than 50 -fold increase in epoxidation ( $\mathrm{pH}$ 4.5) and peroxidation ( $\mathrm{pH} 4.5$ ) rates, respectively, while essentially abolishing dismutation ( $\mathrm{pH} 4.5)$ activity. In addition, the chlorination ( $\mathrm{pH} 2.75)$ activity of the mutant was only about $0.5 \%$ of the original activity of CPO (Fig. 2.24).

Catalysis of heme proteins is generally influenced by such factors as heme iron redox potential, bond length between iron and proximal ligand, active site polarity, and accessibility of the heme to substrates. Inability of $\mathrm{C} 29 \mathrm{H}$ to carry out chlorination and dismutation reactions could be caused by the change in the redox potential of iron (233).

Since thiolate ligation provides a stronger push than less electronegative imidazole, the substitution of Cys with a His is expected to increase the redox potential of the heme iron (234). However, a mere substitution of a proximal ligand for a less electronegative residue does not necessarily result in redox potential increase since multiple factors 
determine activity. For example, C357H P450cam loses its oxygenase activity upon substitution of Cys 357 with a His $(208,209)$ while the activity of myoglobin is increased when proximal His is replaced with a Cys $(196,196)$.

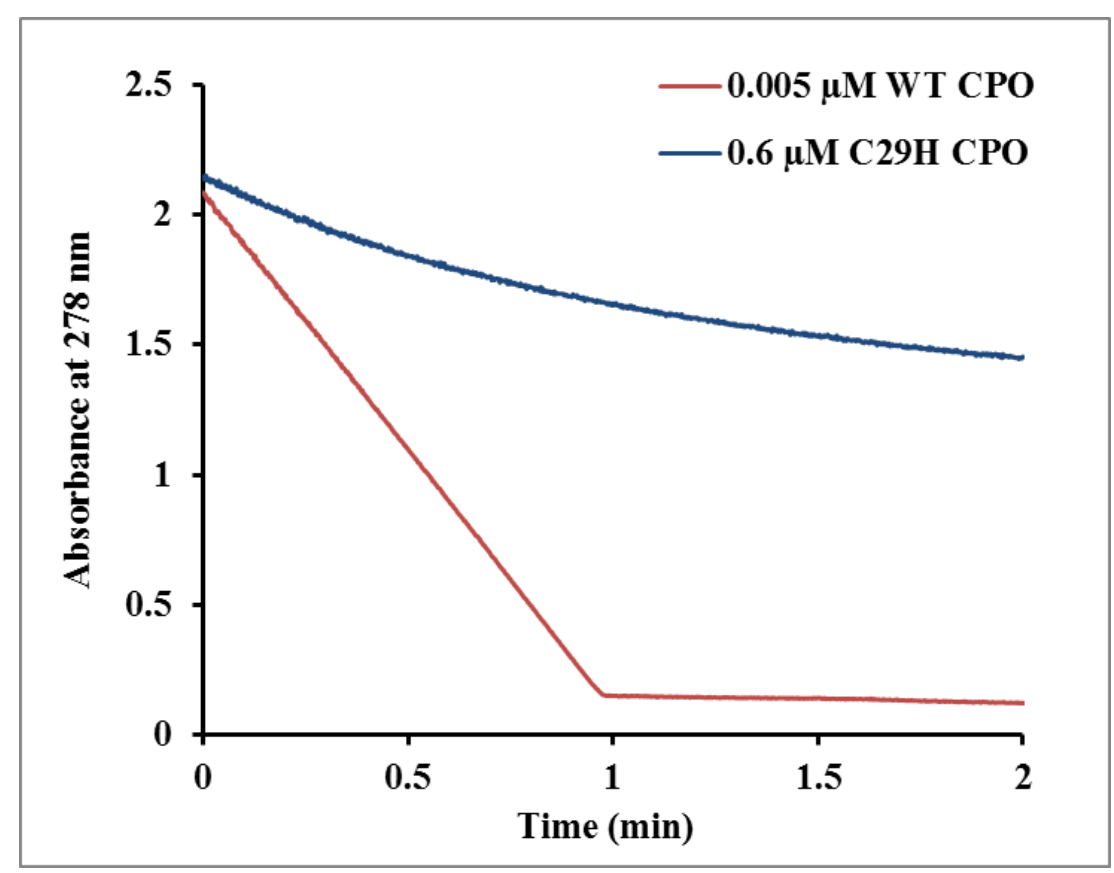

Figure 2.20. Chlorination of MCD to DCD by WT and C29H CPO at pH 2.75.

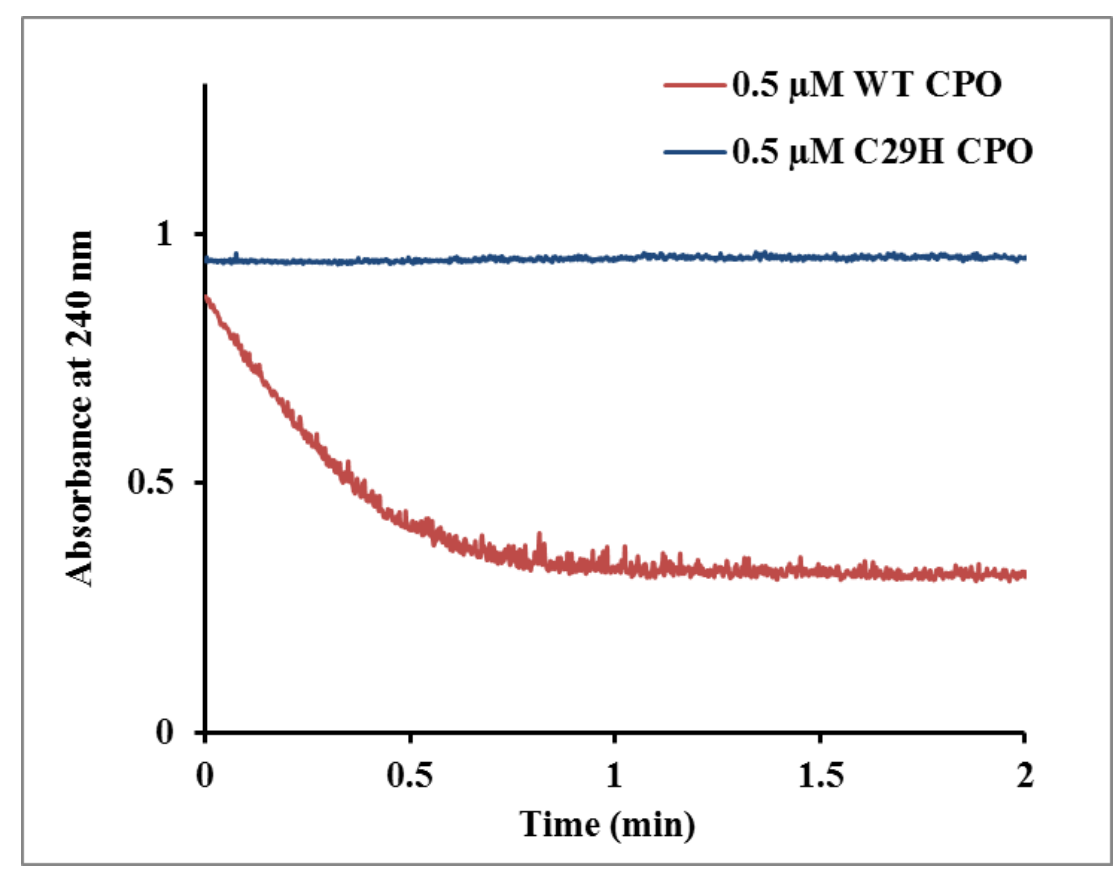

Figure 2.21. Dismutation of $\mathrm{H}_{2} \mathrm{O}_{2}$ by WT and C29H CPO at $\mathrm{pH} 4.5$. 


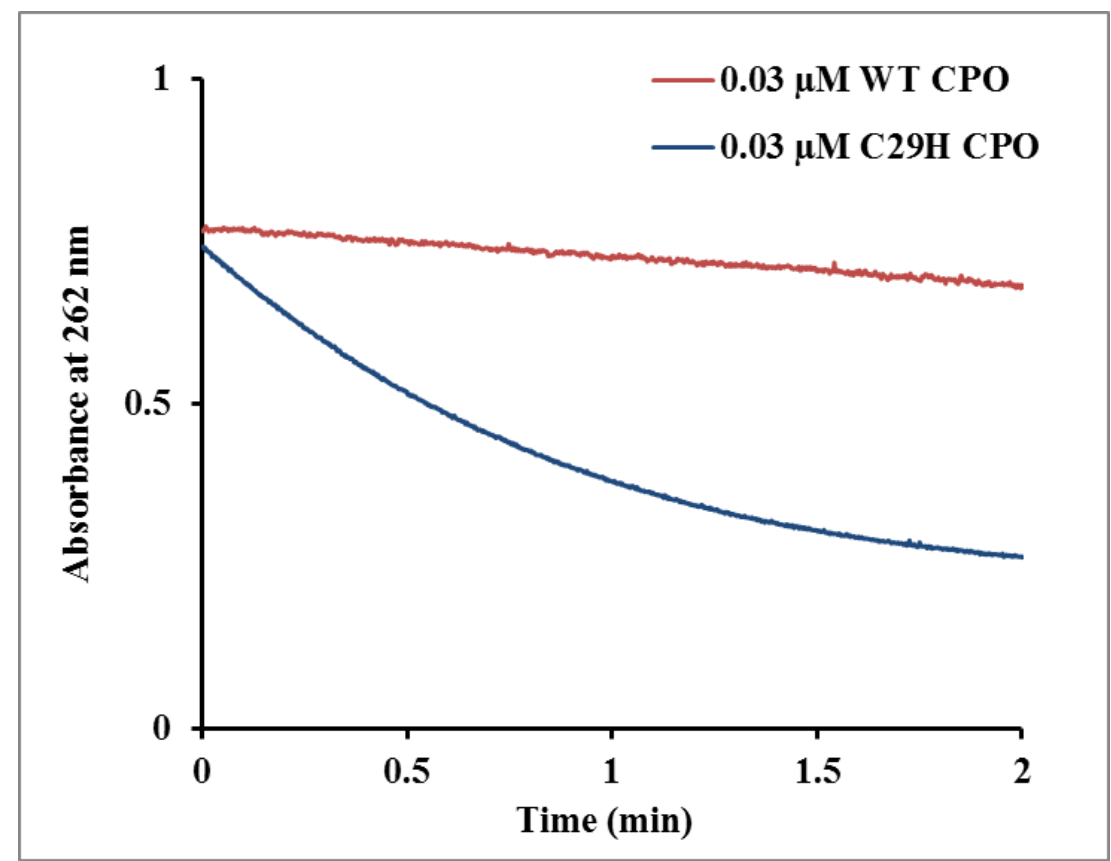

Figure 2.22. Epoxidation of styrene by WT and C29H CPO at pH 4.5.

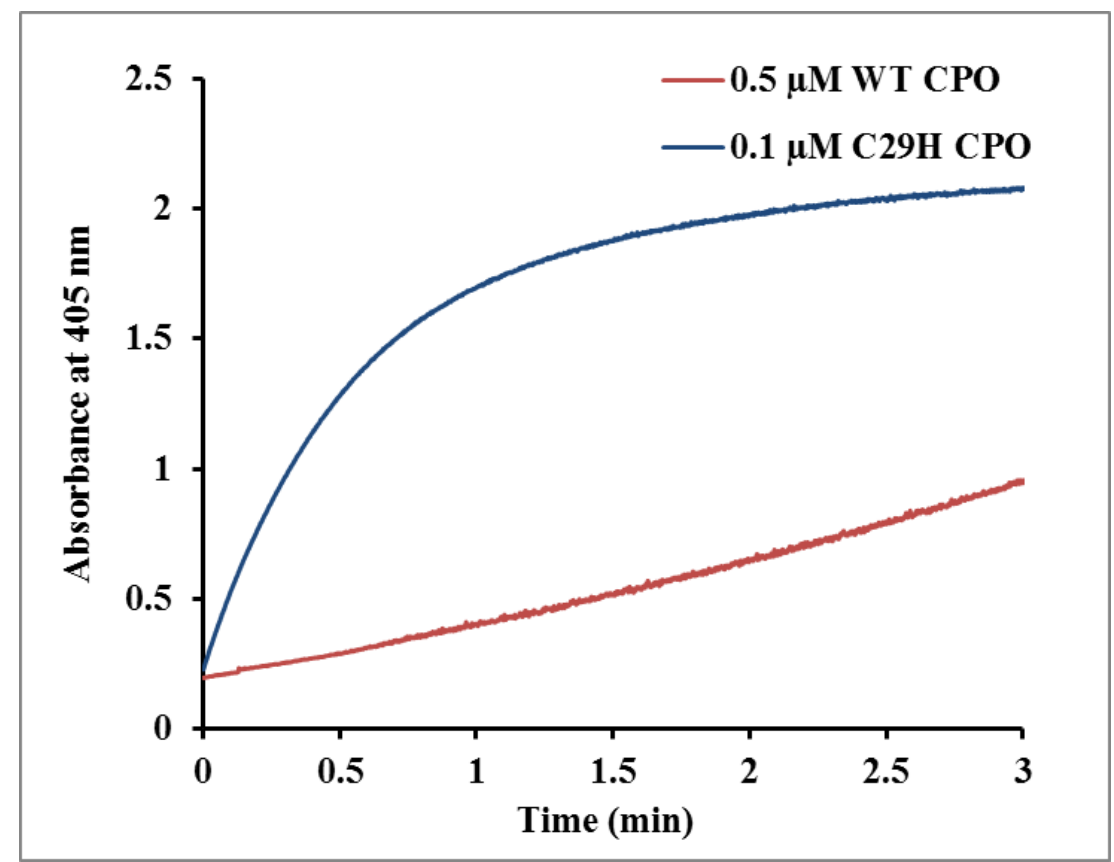

Figure 2.23. ABTS peroxidation by WT and $\mathrm{C} 29 \mathrm{H} \mathrm{CPO}$ at $\mathrm{pH} 4.5$.

My results on $\mathrm{C} 29 \mathrm{H}$ catalysis suggest a decrease in the redox potential of the mutant, which in turn would lead to changes in the distal axial ligand pKa, important for the formation of the active catalytic intermediates (Cpd I, Cpd II, Cpd X) (109, 204). Redox 
potential and basicity of the distal ligand have an inverse relationship $(235,236)$, so increase in the ligand basicity would be expected. A more basic distal ligand is easier to protonate, which partly explains the increase in peroxidation activity, which requires Cpd II formation. It is possible that WT-Cpd II is harder to protonate at $\mathrm{pH} 4.5$, resulting in lower activity than in the mutant, whose Cpd II pKa is higher. Similarly, lack of dismutation activity can be explained by changes in a substrate pKa that might prevent binding of hydrogen peroxide to Cpd I.

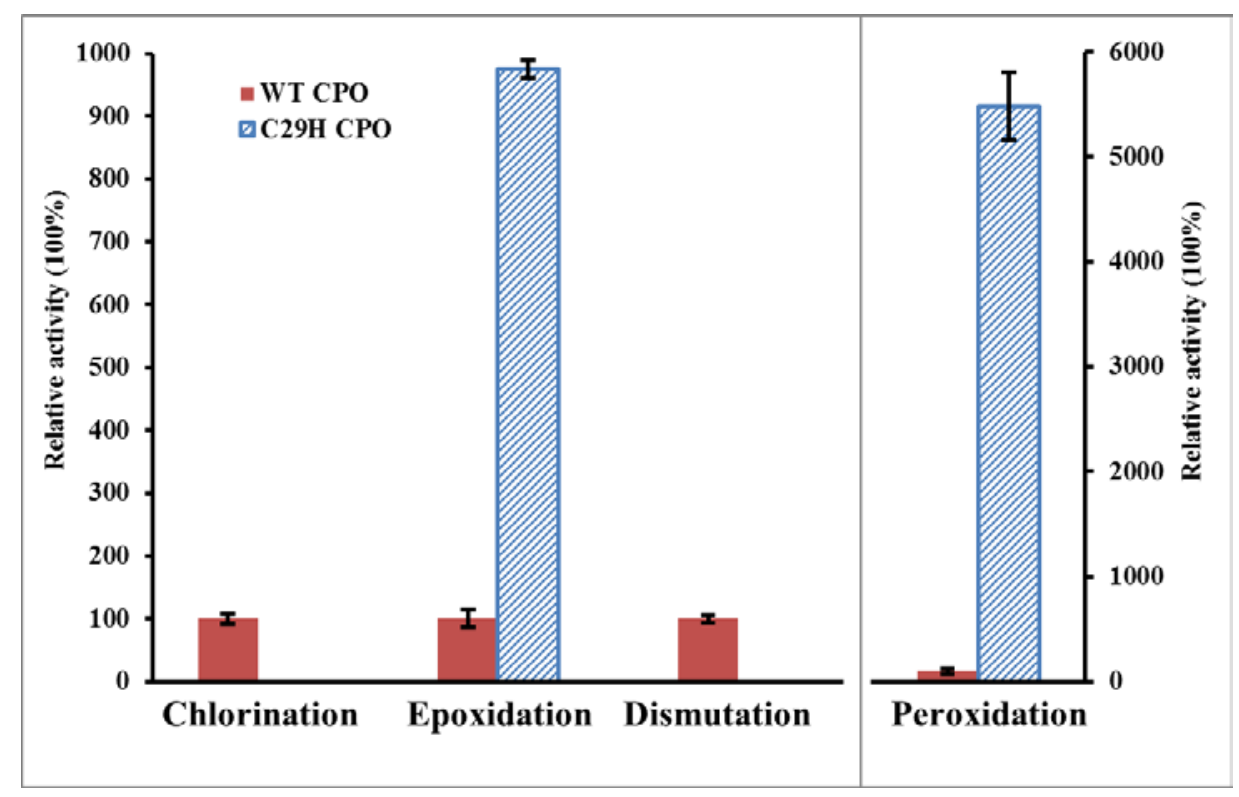

Figure 2.24. Relative activities of $\mathrm{WT}(\mathrm{RZ}=1.3-1.5)$ and $\mathrm{C} 29 \mathrm{H} \mathrm{CPO}(\mathrm{RZ}=1.2-1.5)$ in chlorination ( $\mathrm{pH} 2.75$ ), epoxidation ( $\mathrm{pH} 4.5)$, dismutation ( $\mathrm{pH} 4.5)$, and peroxidation $(\mathrm{pH}$ 4.5) assays.

The lack of dismutation activity in the mutant is expected to contribute to the increase in peroxidation and epoxidation rates, since there is no depletion of Cpd I and oxidizing agent (peroxide) via the dismutation pathway. Another possible contribution to the increase in epoxidation activity is better heme center accessibility by substrate, which is important for two-electron oxidations by heme enzymes (237). The astounding increase in ABTS 
oxidation rates could be a result of perturbations in the active site affecting the heme-edge accessibility required for one-electron oxidations (29). Similar observations were made in cytochrome P450 by Auclair et al., where the Cys $357 \rightarrow$ His mutation resulted in a novel peroxidase activity of the enzyme (30). My results indicate that thiolate ligation is required for dismutation and chlorination activities of CPO, but is not important for peroxidation and epoxidation reactions.

\subsection{7 $\mathrm{pH}$ profiles of catalytic activities of WT and C29H CPO}

Catalytic properties of $\mathrm{CPO}$ are highly $\mathrm{pH}$ dependent. To investigate how $\mathrm{pH}$ affects reaction rates of both WT and C29H CPO, four assays discussed above were performed at various $\mathrm{pH}$. No significant chlorination or dismutation activities were observed for $\mathrm{C} 29 \mathrm{H}$ at any $\mathrm{pH}$ checked (Figs. 2.25 and 2.26). As discussed in the previous section, C29H may be unable to form the protonated Cpd X and Cpd I-peroxide species required for chlorination and dismutation reactions, respectively.

Interestingly, C29H maintained both epoxidase and peroxidase activities over a much wider $\mathrm{pH}$ range than the WT (Figs. 2.27 and 2.28). The rates of the epoxidation reaction catalyzed by WT CPO do not fluctuate significantly over the entire active $\mathrm{pH}$ range, suggesting little influence of $\mathrm{pH}$ on the epoxidation activity of the WT. However, a clear optimum of $\mathrm{pH} 4.5$ is observed for $\mathrm{C} 29 \mathrm{H} \mathrm{CPO}$. Such a $\mathrm{pH}$ profile is consistent with the fact that abolishing dismutation activity should contribute to the increase in epoxidation, and since the highest dismutation activity of the $\mathrm{WT}$ is at $\mathrm{pH} 4.5$, the largest increase is observed at that particular $\mathrm{pH}$. Alternatively, epoxidation reactions catalyzed by WT and C29H CPO might proceed via a dual pathway involving different active intermediates, as has been suggested for the epoxidations catalyzed by CYPs (149). 


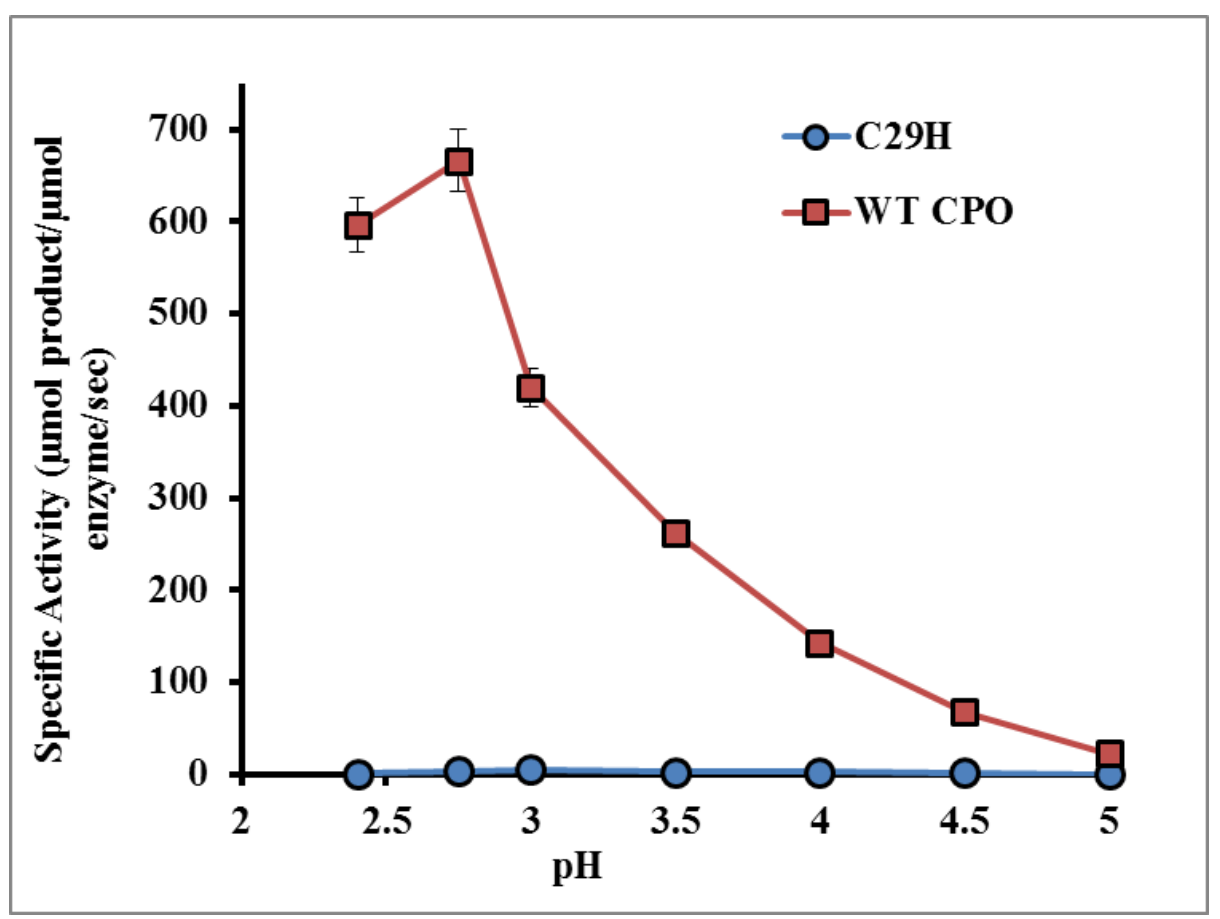

Figure 2.25. $\mathrm{pH}$ profile of chlorination activities of WT and C29H CPO (some error bars are smaller than point size and are not visible on the graph).

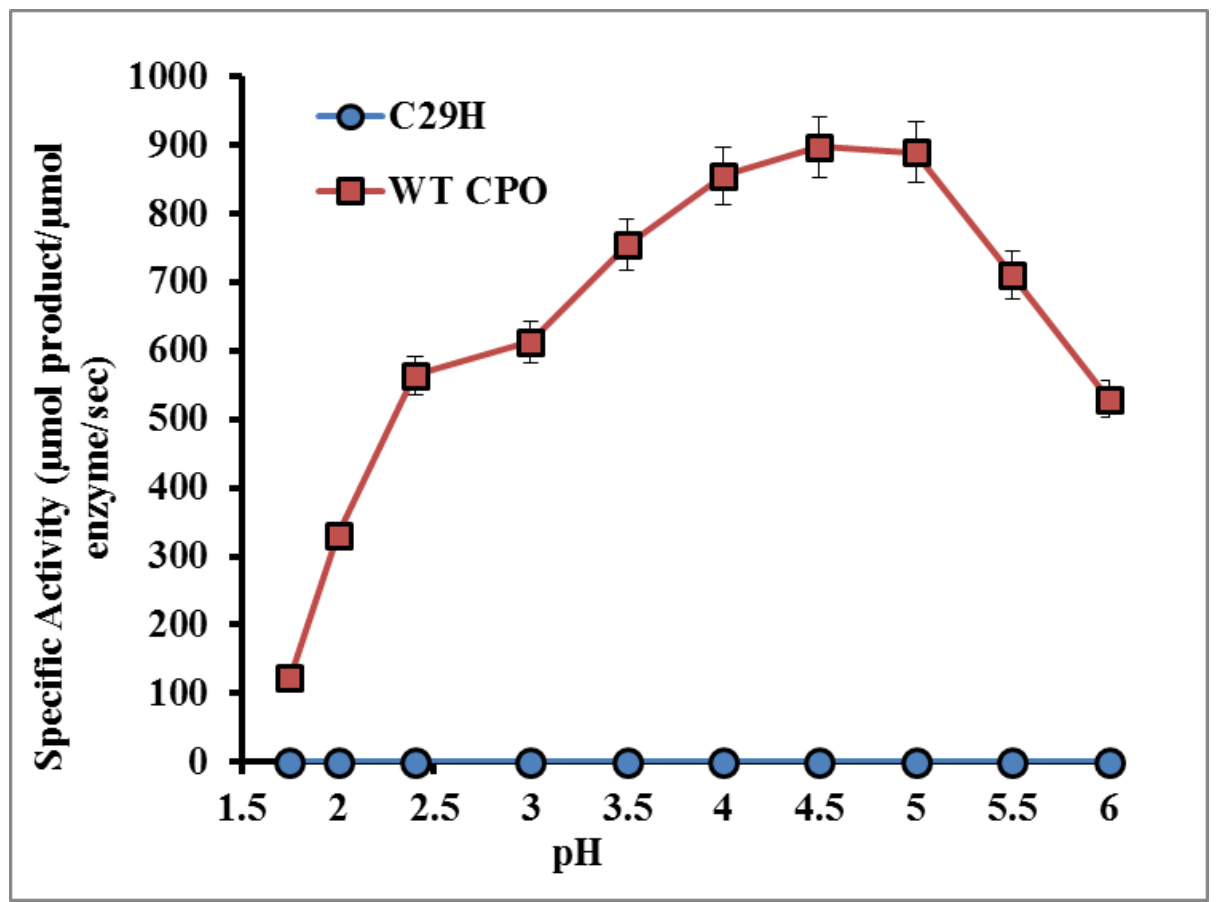

Figure 2.26. $\mathrm{pH}$ profile of dismutation activities of WT and C29H CPO (some error bars are smaller than point size and are not visible on the graph). 


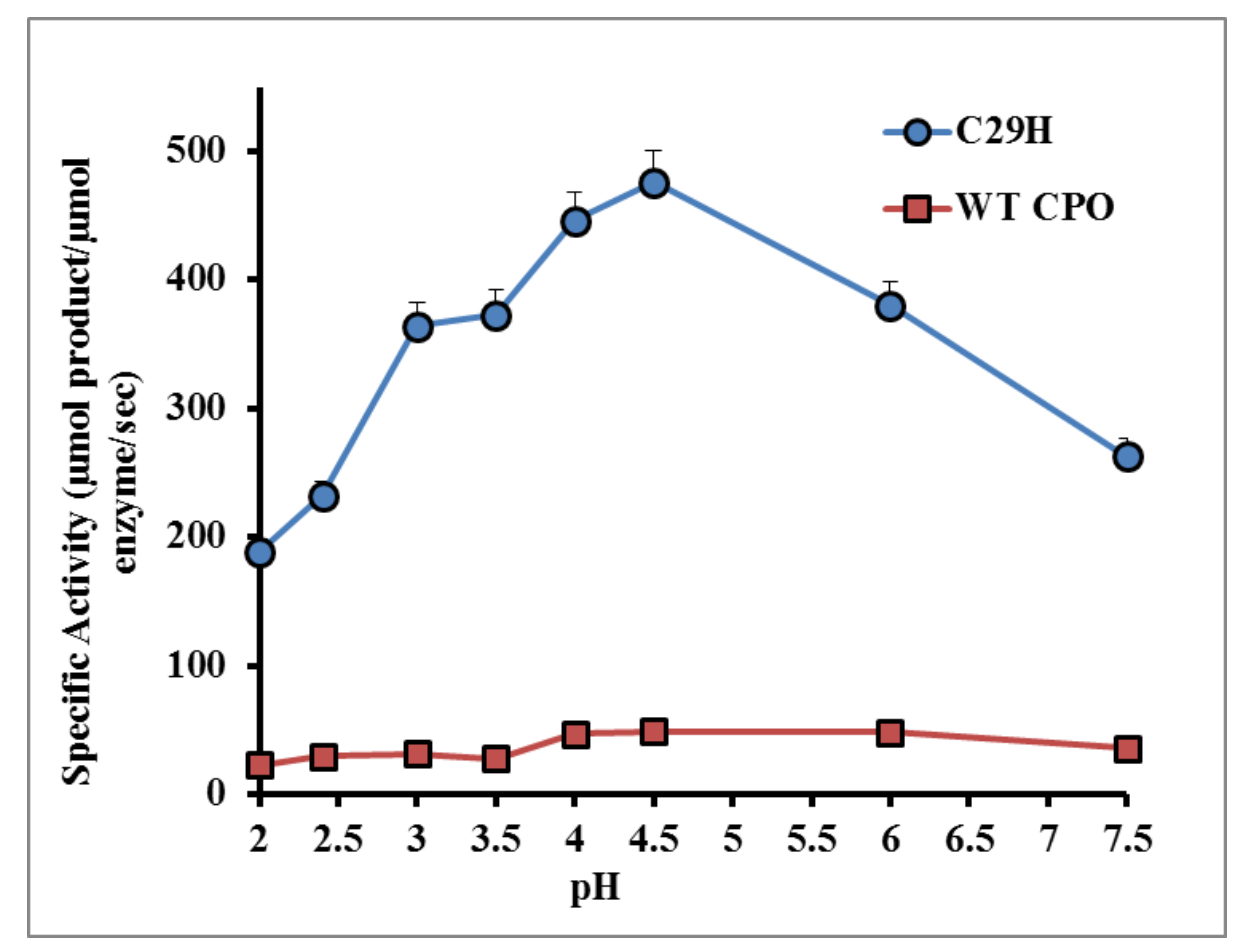

Figure 2.27. $\mathrm{pH}$ profile of epoxidation activities of WT and C29H CPO (some error bars are smaller than point size and are not visible on the graph).

In the peroxidation assay, the WT CPO reaction rates are significantly reduced above $\mathrm{pH} 3.5$ while the mutant remains active even at $\mathrm{pH}$ 5.5. Since Cpd I is the active species in the catalytic cycle of $\mathrm{CPO}, \mathrm{C} 29 \mathrm{H}$ must be able to form this intermediate at both acidic and neutral $\mathrm{pH}$ in contrast to the WT, which loses activity at neutral $\mathrm{pH}$ because of the formation of the inactive alkaline form (228). C29H-Cpd I formation at higher $\mathrm{pH}$ can be explained by the fact that the mutant does not undergo alkaline transition near neutral $\mathrm{pH}$ as observed in the WT. In addition, if Cpd II, the second intermediate in the peroxidation pathway, is indeed an iron(IV)-hydroxide intermediate as suggested by Green and Lai (107), then its pKa would affect the optimal $\mathrm{pH}$. WT is active in the peroxidation assay at very acidic $\mathrm{pH}$, suggesting that the pKa of WT-Cpd II is below 3.25. In C29H, the pKa of Cpd II might be significantly higher (around 4-4.5), and that would result in the formation 
of the active protonated Cpd II at higher $\mathrm{pH}$. For this reason, it is understandable that $\mathrm{C} 29 \mathrm{H}$ CPO is able to carry out peroxidation over a wider $\mathrm{pH}$ range than the WT.

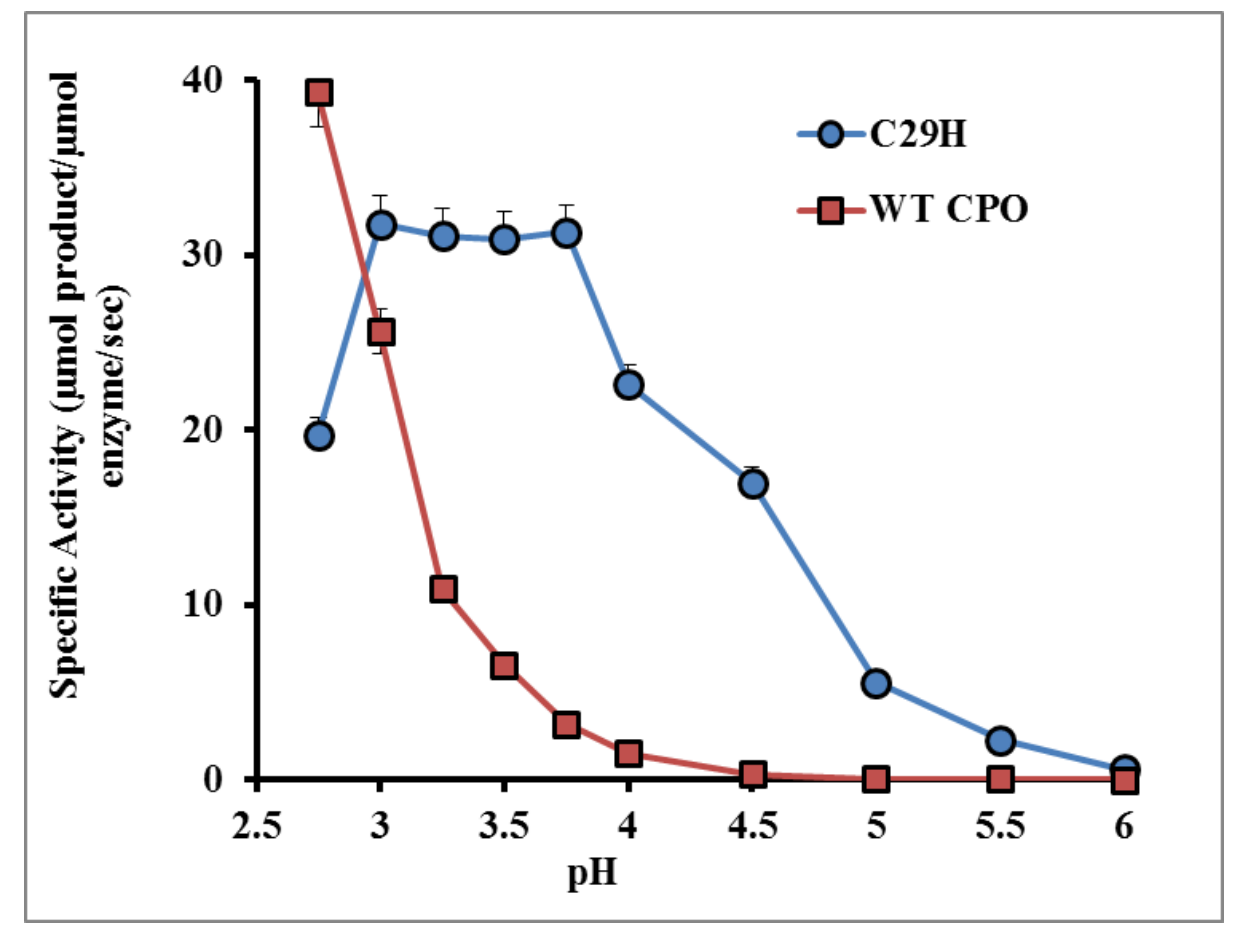

Figure 2.28. $\mathrm{pH}$ profile of peroxidation activities of WT and C29H CPO (some error bars are smaller than point size and are not visible on the graph).

Overall, this study suggests that thiolate ligation plays an important role in both the structure and catalysis of CPO, and substitution with a histidine affects the biochemical properties of the enzyme not only because of the different natures of the ligands but also because of possible changes in the protein architecture. Although, secondary structures of the WT and C29H did not differ significantly as measured by CD, it is possible that tertiary changes have affected the heme environment (for example, the high to low spin conversion and the change in the heme coordination), which is important for the enzyme function. Furthermore, my results on CO binding to single, double, and triple Cys mutants have shown that proximal thiolate ligation is not required for P450-type spectroscopic properties 
of CPO. However, proximal thiolate is important for binding of anionic ligands.

\subsection{Conclusion}

After years of research on heme catalysis, there is still an ongoing debate about the importance of the proximal thiolate ligation in heme proteins. Several proximal ligand mutant heme proteins were engineered and characterized by different researchers with varying success, and no consensus on the role of proximal Cys has been achieved. In this study, I employed rational design to investigate the role of Cys 29 in biochemical and physical properties of CPO. Three CPO mutants (C29H, C29H/C79H, C29H/C79H/C87H) with proximal imidazole ligation were constructed using site-directed mutagenesis and characterized using various spectroscopic techniques. My study has demonstrated that proximal thiolate ligation is important for the catalytic diversity of CPO, particularly for chlorination and dismutation activities. However, it is not essential for peroxidation and epoxidation. Furthermore, I discovered that thiolate ligation is not a prerequisite for the P450-like spectroscopic signature of CPO. This finding is quite unexpected since it has been proposed that proximal Cys ligation in CYPs and CPO is responsible for the unique optical property of these proteins. My work has provided solid support for the importance

of thiolate ligation in heme catalysis and has opened new avenues for further exploration of factors defining biochemical properties of heme enzymes. 


\section{CHAPTER III.}

\section{EFFECTS OF HYDROGEN BOND NETWORK IN THE PROXIMAL HELIX ON THE SPECTROSCOPIC PROPERTIES OF CHLOROPEROXIDASE}

\subsection{Background}

Despite extensive research performed on various heme-thiolate enzymes including CYPs, CPO, and NO synthase, much remains to be explored about mechanisms influencing their catalysis $(28,31-33)$. Various spectroscopic techniques along with site-directed mutagenesis are utilized to obtain information about how particular protein structural features influence its function. It has been shown that the nature of the proximal and distal ligands as well as hydrogen bonding networks in the active site play important roles in defining the biochemical and physical properties of heme proteins. In general, the redox potentials of heme proteins decrease with more electronegative proximal ligands creating the following trend: imidazole $>$ thiolate $>$ phenolate (48). However, proximal imidazole ligation does not guarantee higher redox potential since the overall architecture of the active site fine-tunes reactivity. For example, the redox potentials of CPO, a heme thiolate protein, and HRP, a heme imidazolate enzyme, are $-140 \mathrm{mV}$ and $-278 \mathrm{mV}$ at $\mathrm{pH} 7$, respectively (48), which implicates the critical role of hydrogen bonding networks within the active site in determining the structural and functional properties of heme proteins.

In the proximal site of CPO, there are $2 \mathrm{H}$-bonds between Cys 29 and the amide groups of Ala 31 and Leu 32 situated at the N-terminus of helix A (98). These H-bonds prevent protonation of the thiolate, which results in inactivation of CPO, and decrease its negative charge thus contributing to the increase of the iron redox potential $(101,102)$. In addition to these H-bonds, there is another H-bonding network formed by Arg 26-Asn 37 and Ala 
27-Asn 33, which is a part of helix A of CPO (Fig. 3.1). The effects of this H-bonding network on the spectroscopic and catalytic properties of CPO are unknown.

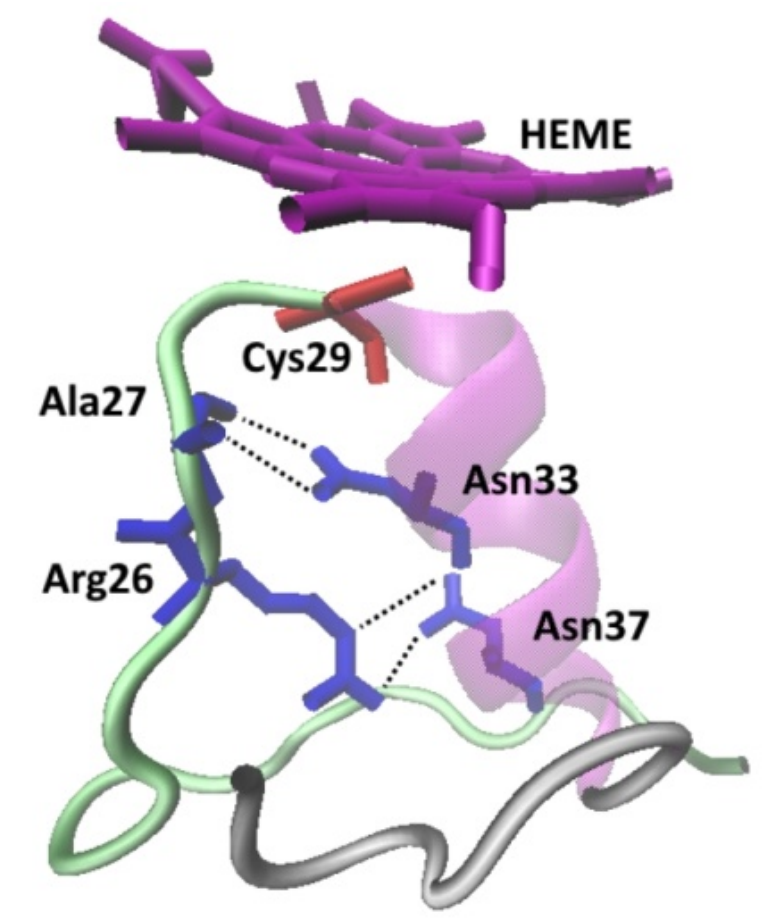

Figure 3.1. Hydrogen bond network in the proximal helix of CPO.

In this study, I explored how the partial or complete removal of the aforementioned H-bonds affected spectroscopic properties of CPO. I replaced Arg 26 and Asn 33 with Ala to disrupt H-bonding and characterized R26A, N33A, and R26A/N33A CPO using UVVis spectrophotometry and CD spectroscopy.

Electronic absorption spectroscopy, or UV-Vis spectrophotometry, is often used to probe the heme environment and its electronic structure, i. e., the coordination and spin state of the heme iron. As described in the introduction, a typical heme protein absorption spectrum (Fig. 18) (177) displays the Soret, $\delta$, and Q $(\alpha, \beta, \mathrm{CT})$ bands that result from electronic transitions in the porphyrin (Fig. 19) (178). Shifts and shapes of these bands can provide information about the spin and coordination state of the heme iron. The Soret and 
Q bands at lower wavelengths as well as a charge transfer band at around $650 \mathrm{~nm}$ indicate that the heme iron is in a high spin state. Furthermore, the intensities of the $\alpha$ and $\beta$ bands relative to each other can provide information about relative energies of the $a_{2 u}$ and $a_{1 u}$ orbitals of the porphyrin. For example, if both orbitals are very similar in energy, the $\alpha / \beta$ intensity ratio will be small. On the contrary, if the $\mathrm{a}_{2 u}$ and $\mathrm{a}_{1 u}$ orbitals have different energies, the $\alpha / \beta$ intensity ratio will be large (179).

Other interactions that can be observed with UV-Vis spectrophotometry include porphyrin-iron and iron-ligand interactions. For example, ferrous iron has filled $\mathrm{d} \pi$ orbitals $\left(d^{6}\right)$, and electrons in $d \pi(d(x z), d(y z))$ orbitals of the iron strongly interact with electrons in porphyrin $\pi^{*}\left(\mathrm{a}_{1 \mathrm{u}}\right)$ orbital. Such strong interactions result in a larger separation between porphyrin $\pi$ and $\pi^{*}\left(\mathrm{a}_{2 \mathrm{u}}\right.$ and $\left.\mathrm{a}_{1 \mathrm{u}}\right)$ orbitals causing the hypsochromic (blue) shifts in electronic spectra (Fig. 20). In contrast, in metalloporphyrins with closed d-shells ( $\mathrm{d}^{0}$ or $\left.\mathrm{d}^{10}\right)$, the $\mathrm{d} \pi$ metal orbitals have little effect on the $\pi$ to $\pi^{*}$ energy separation in porphyrin electronic spectra because of their low energy (180). Ligand-binding studies can provide useful information about the environment of the active site and insights about catalytic mechanisms. Frequently, binding of a ligand in the heme distal side causes changes of the iron spin state. For example, CPO, whose resting state is a five-coordinate high spin form, changes to the low spin upon binding cyanide.

Circular dichroism is often used to investigate protein secondary and tertiary structures and to monitor structural changes in mutant proteins or protein-ligand complexes. Several algorithms are available for estimation of alpha-helical, beta-sheet, and random coil contents and include CONTINLL $(184,185)$, SELCON3 $(186,187)$, CDSSTR (188-190), VARSLC $(188,189)$, and K2D (191). One method might be more suitable than 
others for the analysis of a particular protein since the aforementioned algorithms utilize different protein reference sets. For example, when analyzing globins, it is prudent to choose a program that has more globular proteins in its reference set so that better prediction of the protein structure can be made. In the case of CPO, I found that CDSSTR program produced more accurate estimations of secondary structures, especially for alphahelices that were in a better agreement with the published crystal structure of CPO.

Circular dichroism can provide useful information about integrity of protein domains expressed separately, protein stability under changing conditions, and structural alterations in mutants or upon ligand binding. For heme proteins, CD may also reflect changes in the environment of the heme (192). For example, heme absorbs strongly at around $410 \mathrm{~nm}$ and has additional signals between 500 and $600 \mathrm{~nm}$. Thus, changes in those regions would indicate changes in the heme active site (238).

In this study, I utilized the techniques described above to probe the roles of proximal H-bonds in CPO. My results have shown that the H-bond network of the proximal helix is important not only for maintaining a local active site environment but also for the overall organization of $\mathrm{CPO}$ that in turn affects ligand binding.

\subsection{Experimental procedures}

All chemicals and materials used are the same as described in section 2.2.1.

\subsubsection{Construction of R26A, N33A, and R26A/N33A CPO genes}

To investigate the structural and spectroscopic roles of the hydrogen bond network in the proximal helix of CPO (Arg 26-Asn 37 and Ala 27-Asn 33), two single (R26A, N33A) mutants and one double (R26A/N33A) mutant were created. Mutations were introduced into the pCPO3.I - AmdS (Fig. 2.1) via PCR amplification using the QuikChange II XL 
site-directed mutagenesis kit. Polymerase chain reaction was carried out as described in section 2.2.2. The following primers were used to introduce the R26A, N33A, and R26A/N33A mutations:

Coding strain - R26A primer 1

5’- CTACCGACTCTGCTGCTCCTTGCCCAGCTCTGAACG - 3’

Non-coding strain - R26A primer 2

5’- GAGCTGGGCAAGGAGCAGCAGAGTCGGTAGGACCTG - 3’

Coding strain - N33A primer 1

5’- CTTGCCCAGCTCTGGCCGCTCTTGCCAACCACGGTT - 3’

Non-coding strain - N33A primer 2

5’- GTTGGCAAGAGCGGCCAGAGCTGGGCAAGGAGCACGAG - 3’

Coding strain - R26A/N33A primer 1

5’- GTCCTACCGACTCTGCTGCTCCTTGCCCAGCTCTGG-3’

Non-coding strain - R26A/N33A primer 2

5’- CAAGGAGCAGCAGAGTCGGTAGGACCTGGGGCGAC -3’

Polymerase chain reaction products were amplified in DH5- $\alpha$ E. Coli. Mutations were confirmed by DNA sequencing (Figs. 3.2-3.4).

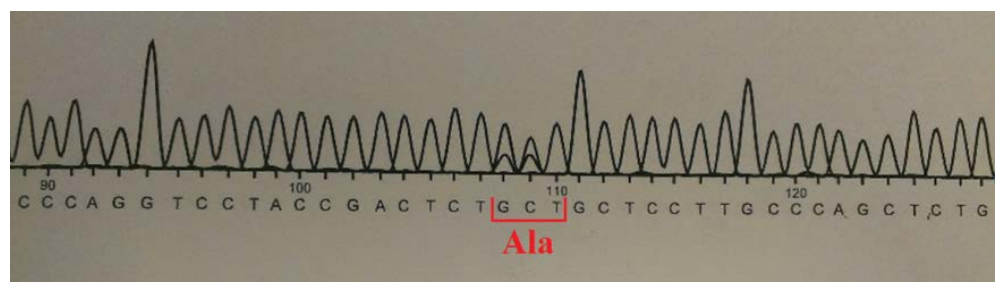

Figure 3.2. R26A pCPO sequencing results. 


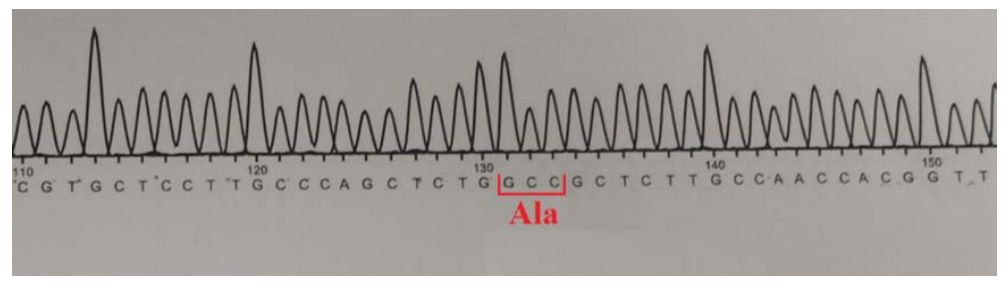

Figure 3.3. N33A pCPO sequencing results.

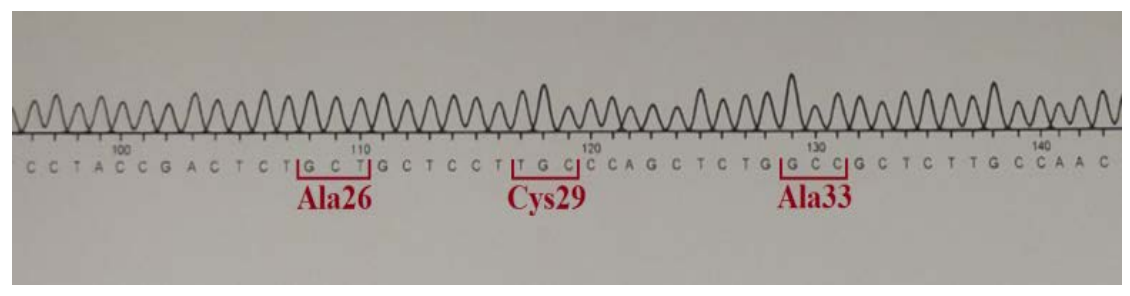

Figure 3.4. R26A/N33A pCPO sequencing results.

\subsubsection{Transformation of Aspergillus niger with mutant pCPO, expression and purification of R26A, N33A, and R26A/N33A CPO}

Transformation of $A$. niger with mutant pCPO was performed as described in section 2.2.3. Expression and purification of mutant $\mathrm{CPO}$ was carried out as described in section 2.2.4 with some modifications. Expression of R26A CPO proved to be quite difficult. Since R26A had low expression levels, $72 \mathrm{~L}$ of culture were grown. After purification, the protein contained both halo- and apoprotein. Hoping to improve incorporation of heme into the R26A mutant, I added hemin to cultures grown at $25^{\circ} \mathrm{C}$ for three days to a final concentration of either $25 \mu \mathrm{M}$ or $50 \mu \mathrm{M}$.

During purification on DEAE column, R26A CPO eluted at approximately 20\% 0.5 $\mathrm{M} \mathrm{NaCl}$. Fractions with RZ values $\left(\mathrm{A}_{420} / \mathrm{A}_{280}\right)$ above 0.5 were used for further purification by size exclusion chromatography (Fig. 3.5). Fractions with RZ values between $1.0-1.1$ were used in subsequent studies. Purity of the mutant CPO was assessed by SDS-PAGE (Figs. 3.6 and 3.7). 


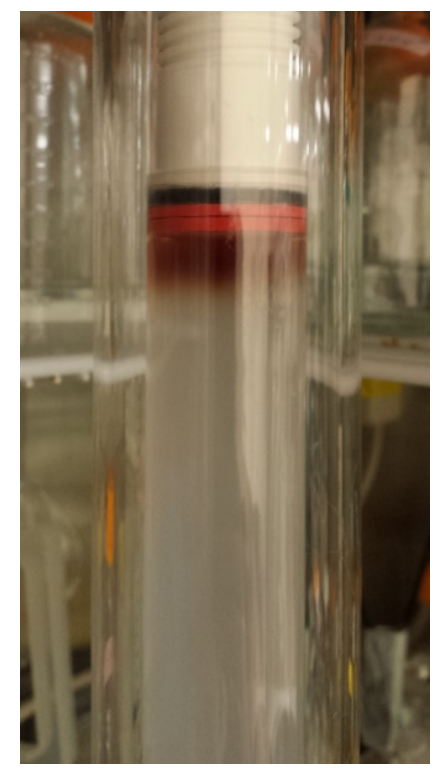

Figure 3.5. R26A CPO sample loaded on a gel filtration column.

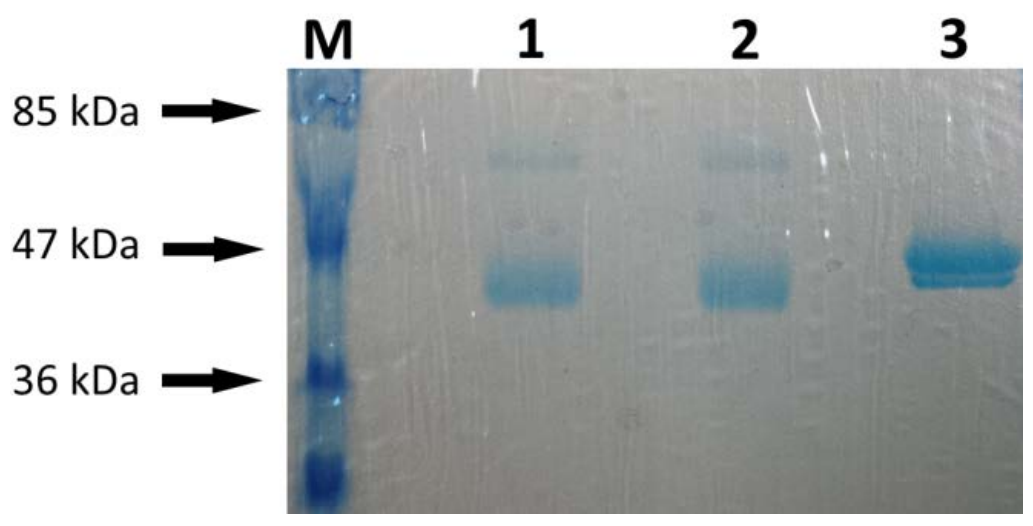

Figure 3.6. SDS-PAGE. WT and R26A CPO. Lanes 1 and 2 - R26A, lane 3 - WT CPO.

Expression of the other two mutants, N33A and R26A/N33A, produced better yields, thus 36-L and 30-L cultures were grown, respectively. During ion-exchange chromatography, N33A and R26A/N33A CPO eluted at approximately 30\% and 20\% 0.5 M NaCl, respectively. Fractions with $\mathrm{RZ}$ values $\left(\mathrm{A}_{420} / \mathrm{A}_{280}\right)$ above 0.6 and 0.5 , respectively, were used for further purification by size exclusion chromatography. Fractions with RZ values between 1.0 - 1.1 were used in subsequent studies. Purity of the mutant CPO was assessed by SDS-PAGE. 


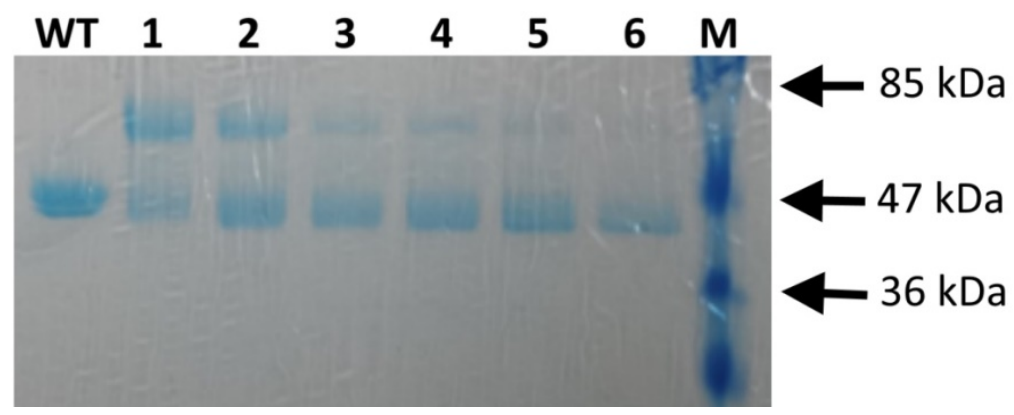

Figure 3.7. SDS-PAGE. N33A CPO. WT - wild type CPO, 2-6 - N33A fractions from gel filtration, $\mathrm{M}-$ marker.

\subsubsection{UV-Visible spectrophotometry}

UV-Visible spectrophotometry was used to investigate heme electronic configuration and ligand-binding properties of the mutants lacking hydrogen bonds in the proximal helix A of CPO. The procedures are described in sections 2.2.5.3 and 2.2.6 with the following modifications. The effect of $\mathrm{pH}$ study was performed at $\mathrm{pH} 2.4,3.5,6.0,8.0,10.0$. Excess cyanide (100 mM) binding was performed at pH 6.0 and $\mathrm{pH} 12.0$. Titrations of CPO (10$800 \mu \mathrm{M}$ for WT and 1-10 mM for mutants) with $\mathrm{CN}^{-}$were performed in a phosphate buffer, $\mathrm{pH}$ 6.0. The cyanide dissociation constant $(\mathrm{Kd})$, which is an inverse of a binding constant (K), was calculated from the following equation (239):

$$
\frac{1}{\Delta A}=\frac{1}{[S]} \times \frac{1}{K \Delta A \infty}+\frac{1}{\Delta A \infty},
$$

where $\Delta A$ is the maximum absorbance difference, $\Delta A \infty$ is the change in absorbance of CPO-CN complete formation, $\mathrm{K}$ is a binding affinity constant, and [S] is the concentration of free $\mathrm{CN}^{-}$. The Hill equation (240) was used to determine the stoichiometry of $\mathrm{CN}^{-}$ binding to mutants. In Eq. 3.2, $h$ is the number of binding sites:

$$
\log \left[\frac{\Delta A}{\Delta A \infty-\Delta A}\right]=h \times \log [S]+\log (K)
$$




\subsubsection{CD spectroscopy}

Circular dichroism spectroscopy was used to probe possible changes in secondary and tertiary structures of mutant CPOs as a result of the proximal helix hydrogen bond network disruption. Procedures for the secondary CD are described in section 2.2.5.2. As for the tertiary CD, the spectra of $16 \mu \mathrm{M}$ R26A, $35 \mu \mathrm{M}$ N33A, $11 \mu \mathrm{M}$ R26A/N33A, and corresponding concentrations of WT CPO in phosphate buffer, $\mathrm{pH} 5.9$ were collected in the $250-600 \mathrm{~nm}$ range at room temperature using 1 -cm quartz cuvettes. Three spectra were averaged for each protein.

\subsection{Results and discussion}

\subsubsection{Secondary and tertiary CD spectroscopy of WT and mutant CPO}

Asn 33 and Asn 37 are hydrogen bonded to Ala 27 and Arg 26, respectively, and are located in proximal helix A. Helix A also contains Cys 29 coordinated to the heme, thus deletion of the H-bonds in either of the aforementioned residue pairs might affect both the local environment of the heme and the alpha-helical structure of CPO. Structural alterations, in turn, could influence catalytic and binding properties of CPO. Removal of all H-bonds in the proximal helix is expected to have a larger effect on the helix A position, possibly influencing its flexibility, which also might affect the proximal site environment.

The secondary CD spectra of the single amino acid mutants, R26A and N33A, show only slight changes while the CD spectrum of R26A/N33A is significantly different from that of the WT (Fig. 3.8). As estimated by CDSSTR, the alpha helical structures of WT, R26A, N33A, and R26A/N33A are 31\%, 29\%, 30\%, and 51\%, respectively. The betasheets have been estimated to be at $25 \%, 23 \%, 22 \%$, and $18 \%$, respectively. Disruption of the pair of hydrogen bonds between either R26-N37 or A27-N33 does not seem to 
significantly change secondary structure of CPO. However, removal of both pairs of Hbonds results in a noticeable alteration of the alpha-helix (20\%-increase). Since the amino acids directly adjacent to the proximal helix are disordered, it is possible that the absence of the H-bonds promotes organization of this region into an alpha helix extending helix A affecting its rigidity. Such changes in the proximal helix would affect the local heme environment since Cys 29, the heme ligand, is situated in this helix.

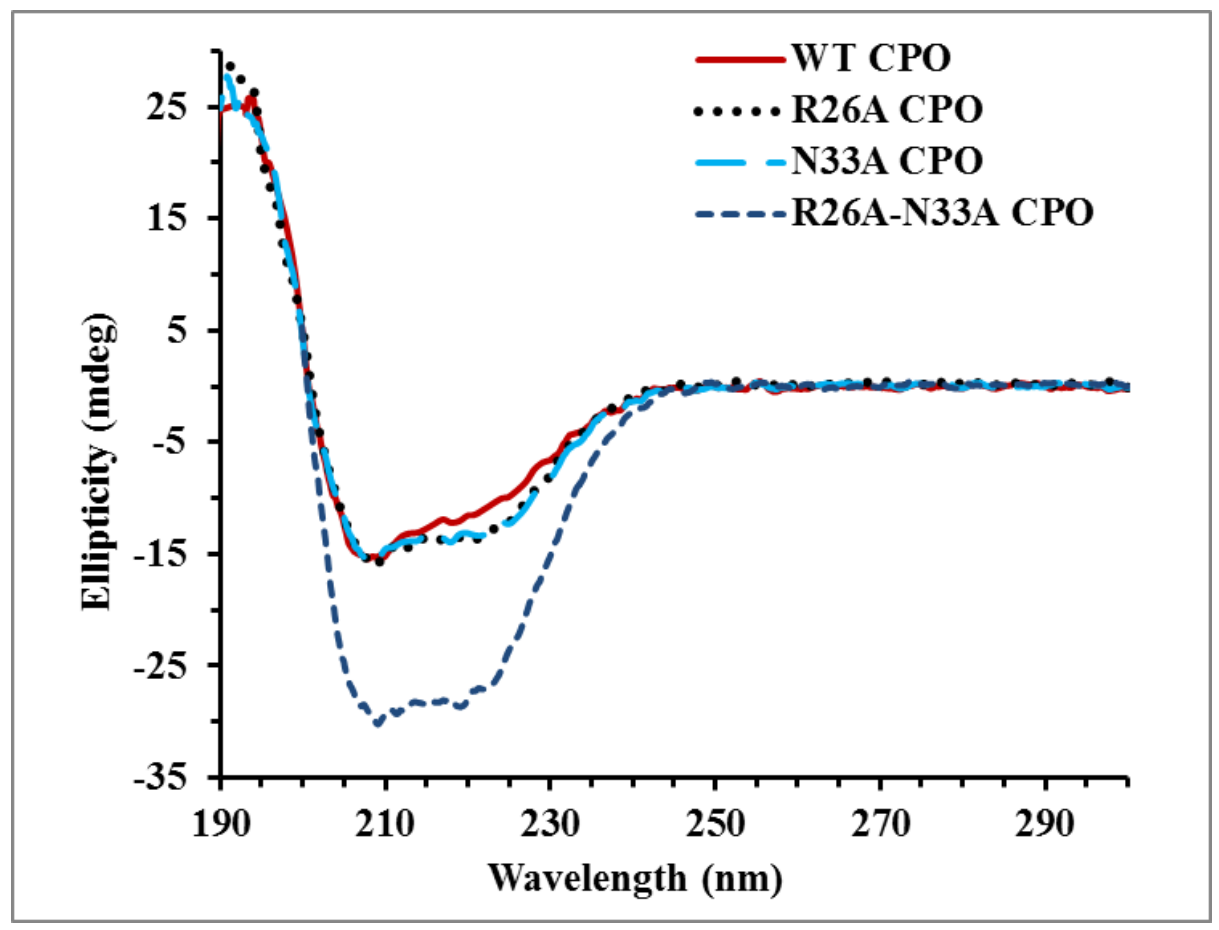

Figure 3.8. Secondary CD of $1.0 \mu \mathrm{M}$ WT and mutant CPO in phosphate buffer, pH 5.9.

To investigate whether mutations in the active site of $\mathrm{CPO}$ affect its tertiary structure, CD spectra of the WT and the mutants have been taken in the 250-600 nm region (Figs. 11-13). All mutants exhibit significant tertiary spectral changes in both aromatic and heme regions. Aromatic residues (Phe, Trp, Tyr) as well as disulfide bonds produce signals between 250 and $330 \mathrm{~nm}$. Trp, Tyr, and Phe absorb at around $290 \mathrm{~nm}, 280 \mathrm{~nm}$, and 260$270 \mathrm{~nm}$, respectively (241). In addition, the disulfide bond band extends from about 330 
$\mathrm{nm}$ to its maximum at around $260 \mathrm{~nm}$ and is usually weak. However, its intensity depends on C-S-S bond angle, dihedral angle of the disulfide bond, and effects of surrounding groups (238). Chloroperoxidase has 18 Phe, 5 Trp, and 11 Tyr in its primary structure. Thus the broad positive signal in 275-300 nm region is likely produced by Trp and Tyr residues. The negative band at around $265 \mathrm{~nm}$ can be a combination of absorptions arising from Phe and a disulfide bond between Cys 79 and Cys 87. All three mutants produce negative signals in aromatic region, which indicate changes in the rigidity or environment of aromatic residues and possibly of the disulfide bond region.

The region between $400-650 \mathrm{~nm}$ provides information about the heme environment. Heme absorbs at around $350 \mathrm{~nm}$ (delta band), $410 \mathrm{~nm}$ (Soret), and between 500-650 nm (Q bands). In the CD spectrum of WT CPO, the heme group has strong absorptions at 428 $\mathrm{nm}$ (Soret) and 545 (Q band) nm. Additional weak negative signals are located at $372 \mathrm{~nm}$ and $480 \mathrm{~nm}$. Spectra of the mutants are markedly different from that of the WT (Figs. 3.93.11 and Table 3.1). The signals described above for the WT disappear in R26A with the exception of a weak absorption at around $480 \mathrm{~nm}$. There is a broad negative peak in 500$600 \mathrm{~nm}$ region with two maxima at $561 \mathrm{~nm}$ and $572 \mathrm{~nm}$. The CD spectrum of R26A suggests that the heme environment in R26A is quite different from that in the WT. In addition, the absence of the Soret signal may reflect poor incorporation of the heme into the R26A mutant, which is also supported by the lower RZ value. Furthermore, the Soret band in R26A UV-Vis spectrum decreases significantly within 1-2 months of protein expression, indicating low mutant stability. A very weak signal at around 344-350 nm (delta band) is present in the spectra of all three mutants, which indicates that the electronegative environment of the heme has changed in H-bond deletion mutants. 


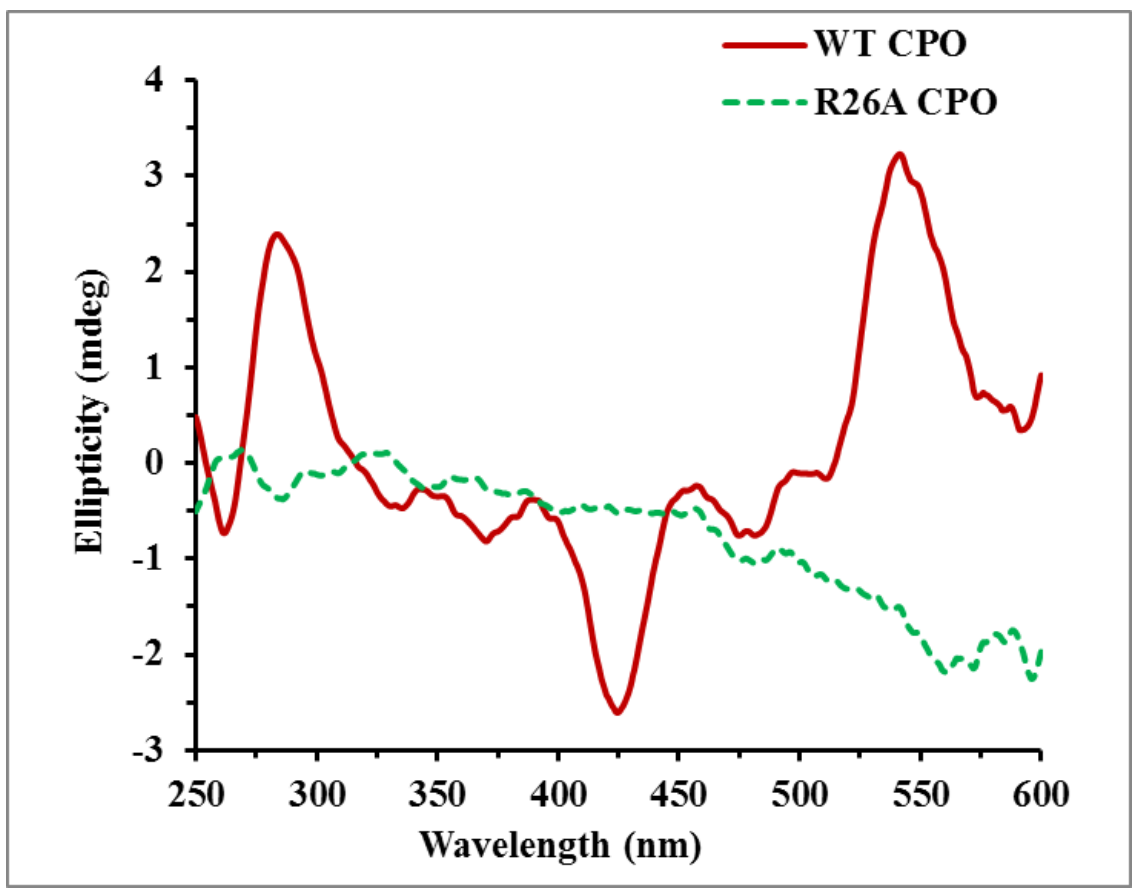

Figure 3.9. Tertiary CD spectra of $16 \mu \mathrm{M}$ WT and R26A CPO at pH 5.9.

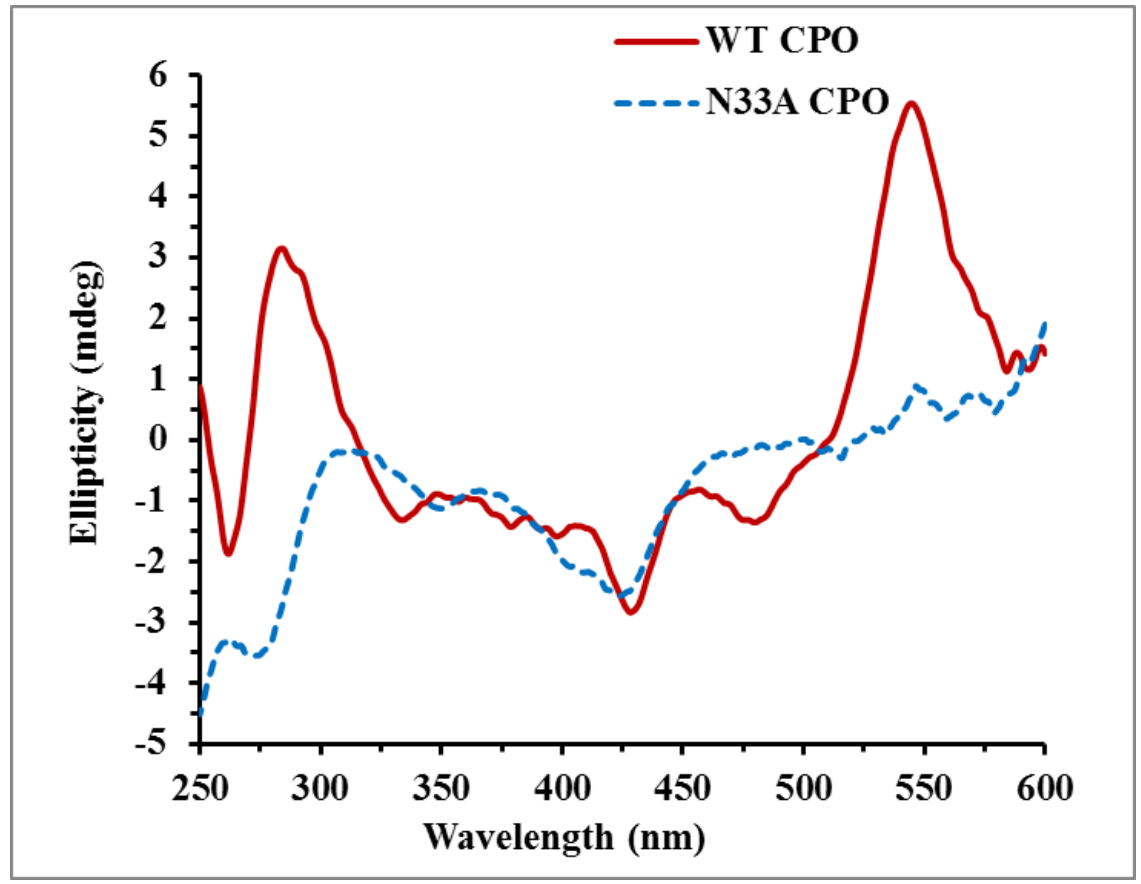

Figure 3.10. Tertiary CD spectra of $35 \mu \mathrm{M}$ WT and N33A CPO at pH 5.9. In N33A and R26A/N33A, the Soret bands are observed at $424 \mathrm{~nm}$ and $420 \mathrm{~nm}$, respectively, and are broader than that of the WT with a shoulder at around $400 \mathrm{~nm}$. 


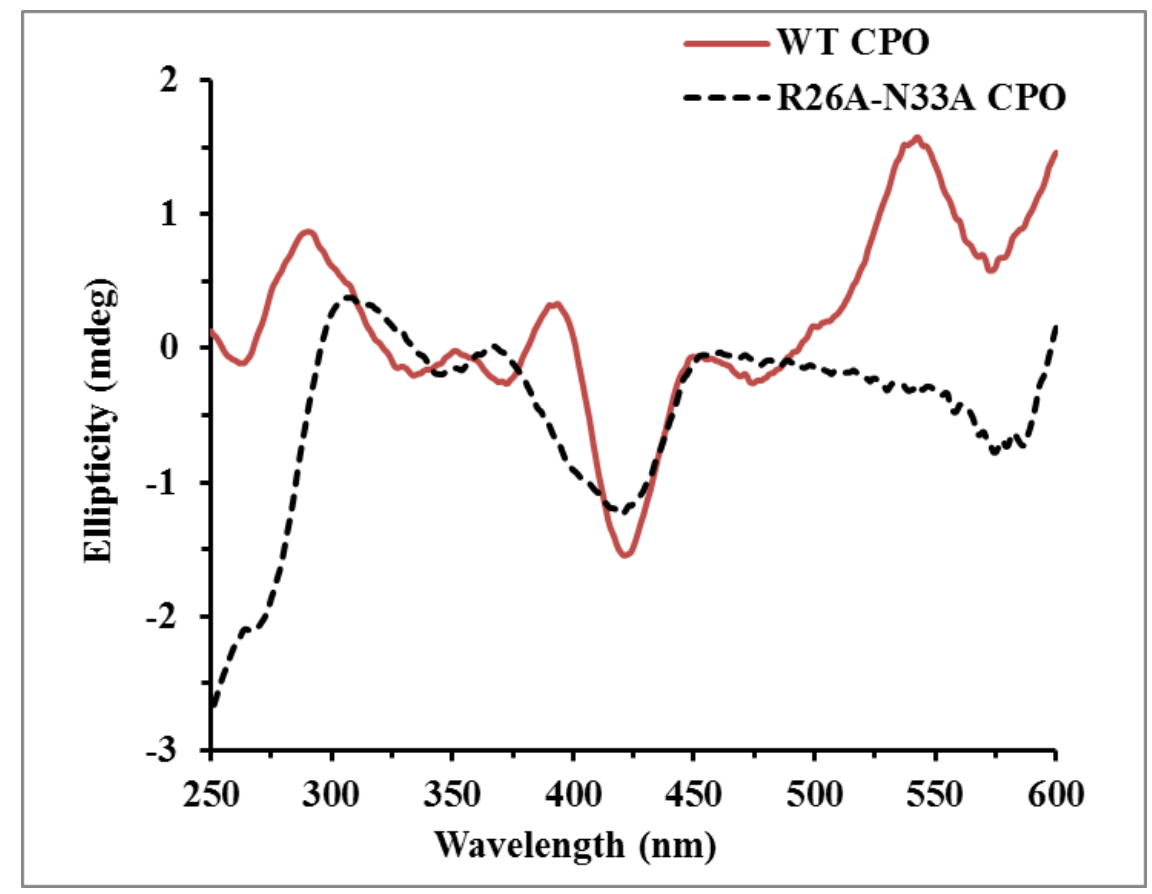

Figure 3.11 Tertiary CD spectra of $11 \mu \mathrm{M}$ WT and R26A-N33A CPO in phosphate buffer, pH 5.9.

Table 3.1. CD spectral properties of ferric WT and mutant CPO in phosphate buffer, $\mathrm{pH}$ 5.9 in the heme region $((+),(-)$, (s) indicate positive and negative signals, and a shoulder in a band, respectively).

\begin{tabular}{lcccc}
\hline Protein & $\boldsymbol{\delta}$ band (nm) & Soret (nm) & $\begin{array}{c}\text { CT band } \\
(\mathbf{n m})\end{array}$ & Q bands (nm) \\
\hline WT CPO & $372(-)$ & $428(--)$ & $480(-)$ & $545(++)$ \\
\hline R26A CPO & $344(-)$ & -- & $481(-)$ & $561(--), 572(--)$ \\
\hline N33A CPO & $350(-)$ & $424(--)($ s) & -- & $547(+), 559(-), 579(-)$ \\
\hline $\begin{array}{l}\text { R26A/N33A } \\
\text { CPO }\end{array}$ & $345(-)$ & $420(--)(s)$ & -- & $575(--), 580(--)$ \\
\hline
\end{tabular}

In the spectra of both N33A and R26A/N33A mutants, there are no signals at $370 \mathrm{~nm}$ and $480 \mathrm{~nm}$; however, three and two Q bands are present, respectively. These observations suggest that the heme active centers in those two mutants are similar to each other but different from that in the WT. 
Overall, the CD results indicate that the largest secondary structural changes have occurred in the R26A/N33A affecting alpha-helical content, while the heme environment has been altered in all three mutants. To further investigate the properties of the heme, UVVis spectroscopy was employed, and the results are discussed in the following section.

\subsubsection{UV-Vis spectroscopic properties of WT, R26A, N33A, and R26A/N33A CPO}

As mentioned before, heme proteins possess a spectroscopic signature in the UVVisible region that can provide useful information about the electronic and molecular structure of the heme active site. The spectrum of WT CPO shows the Soret band at 398 $\mathrm{nm}$ as well as $\beta, \alpha$, and CT signals at 515, 545, and $650 \mathrm{~nm}$, respectively (Figs. 14, 15). The $\beta$ and $\alpha$ bands are not well-resolved indicating greater mixing of the $\mathrm{a}_{2 \mathrm{u}}$ and $\mathrm{a}_{1 \mathrm{u}}$ porphyrin orbitals. The high spin markers at 515 and $650 \mathrm{~nm}$ indicate that the heme iron is in the high-spin pentacoordinate form. The Soret and Q bands of the mutants are red-shifted and their positions are consistent with mutants having a low spin heme (Fig. 3.12 and Table 3.2).

Table 3.2. UV-Vis spectral properties of ferric WT and mutant CPO at pH 5.9 (s-shoulder, n.s.- no shoulder, s.s.- slight shoulder).

\begin{tabular}{lccccc}
\hline Protein & $\begin{array}{c}\delta \text { band } \\
(\mathbf{n m})\end{array}$ & $\begin{array}{c}\text { Soret } \\
(\mathbf{n m})\end{array}$ & $\begin{array}{c}\boldsymbol{\beta} \text { band } \\
(\mathbf{n m})\end{array}$ & $\begin{array}{c}\boldsymbol{\alpha} \text { band } \\
(\mathbf{n m})\end{array}$ & $\begin{array}{c}\text { CT } \\
(\mathbf{n m})\end{array}$ \\
\hline WT CPO & -- & $398($ n.s.) & 515 & 550 & 650 \\
\hline R26A CPO & 354 & $418(\mathrm{~s})$ & 540 & 568 & 644 (low) \\
\hline N33A CPO & 356 & 420 (n.s.) & 540 & 575 & -- \\
\hline R26A/N33A CPO & 356 & 421 (s.s.) & 540 & 575 & 644 (low) \\
\hline
\end{tabular}




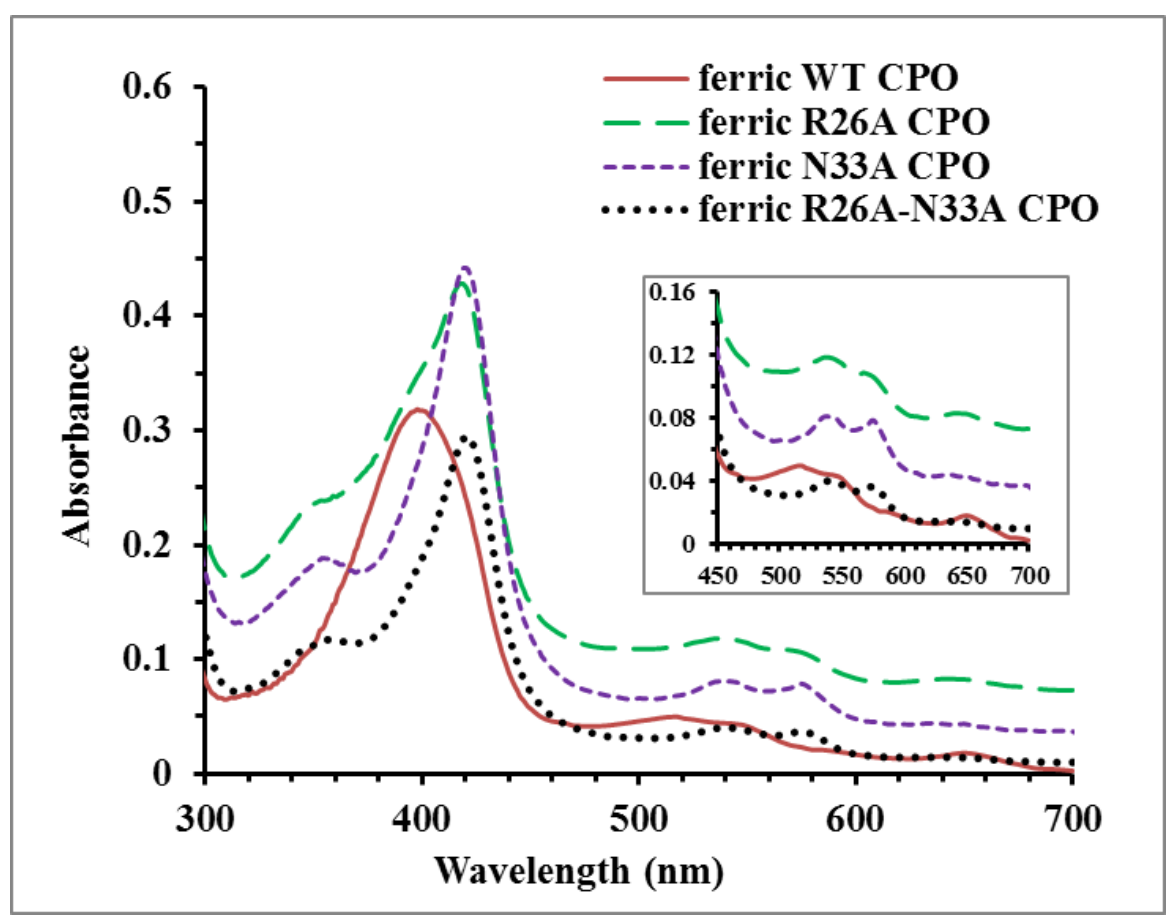

Figure 3.12. UV-Vis spectra of WT, R26A, N33A, and R26A/N33A CPO in $25 \mathrm{mM}$ phosphate buffer, pH 5.9.

The Q bands at around $540 \mathrm{~nm}$ and $570 \mathrm{~nm}$ in the mutant spectra indicate that ferric mutants possess the hexacoordinate low-spin heme with water or an amino acid as a distal ligand. R26A and R26A/N33A show very low absorption peaks at $644 \mathrm{~nm}$, which suggests that a small fraction of the protein might be a high-spin state. Furthermore, the $\alpha / \beta$ band intensity ratio in WT spectrum is similar to that in R26A and lower than that in N33A and R26A/N33A mutant spectra (Fig. 15). These ratios suggest that $\mathrm{a}_{2 u}$ and $\mathrm{a}_{1 \mathrm{u}}$ porphyrin orbitals (Figs. 1.20 and 1.21) in WT and R26A might be closer in energy than those in N33A and R26A/N33A. In addition, the spectra of all mutants possess a clear $\delta$-band, which is also an indication of a low-spin heme.

To investigate structural changes in $\mathrm{CPO}$ at acidic and alkaline $\mathrm{pH}$, the UV-Vis spectra of both WT and mutants were taken at 2.4-10.0 pH range. Wild-type CPO remains in a high spin penta-coordinate form in the acidic $\mathrm{pH}$ range, and converts from the active 
high-spin state to the inactive low-spin form at pH above 7.5 (220). It has been suggested that at this $\mathrm{pH}$, an amino acid in the active site of $\mathrm{CPO}$ is deprotonated, which results in further ionization of other residues. The ionizable amino acid interacts with the heme iron, which influences its pKa. It was also shown that binding of exogenous ligands to the alkaline form of CPO is difficult or impossible (228). These observations indicate that interactions of the ionizable residue with the heme might prevent binding of $\mathrm{H}_{2} \mathrm{O}_{2}$ to heme required for Cpd I formation, thus resulting in inactivation of CPO.

My spectral results are consistent with those reported previously and show that WT $\mathrm{CPO}$ is stable even at very acidic $\mathrm{pH}(2.4)$ and its transition to the alkaline low-spin hexacoordinate form occurs between pH 7 and 8 (Fig. 2.11). Different UV-Vis spectra have been obtained for CPO H-bond mutants, which primarily exist in the low-spin hexacoordinate state in a broad pH range, except R26A (Figs. 3.13-3.17). The spectrum of N33A at pH 2.4 has a broad peak at around $380 \mathrm{~nm}$ without any Q bands present. Such a spectrum resembles that of free heme in aqueous solution, suggesting protein unfolding and exposure of heme to the solvent (242). Another transition occurs above pH 9.0 (Fig. 3.14), which is likely because of the deprotonation of one or more critical residues with an apparent pKa near 10. It is also possible that partial denaturation of the protein occurs because of the high alkalinity. A similar transition is also observed for R26A/N33A at pH10.0 (Fig. 3.16). However, different results are obtained at acidic $\mathrm{pH}$. The shape of the Soret band in R26A/N33A at pH 2.4 suggests that the transition from the low spin has not completely occurred and the double mutant has higher stability at pH 2.4 than N33A (Fig. 3.15). A shoulder at around $400 \mathrm{~nm}$ emerging from the Soret band and a high-spin marker at around $650 \mathrm{~nm}$ indicate the mixed spin state of R26A/N33A. 


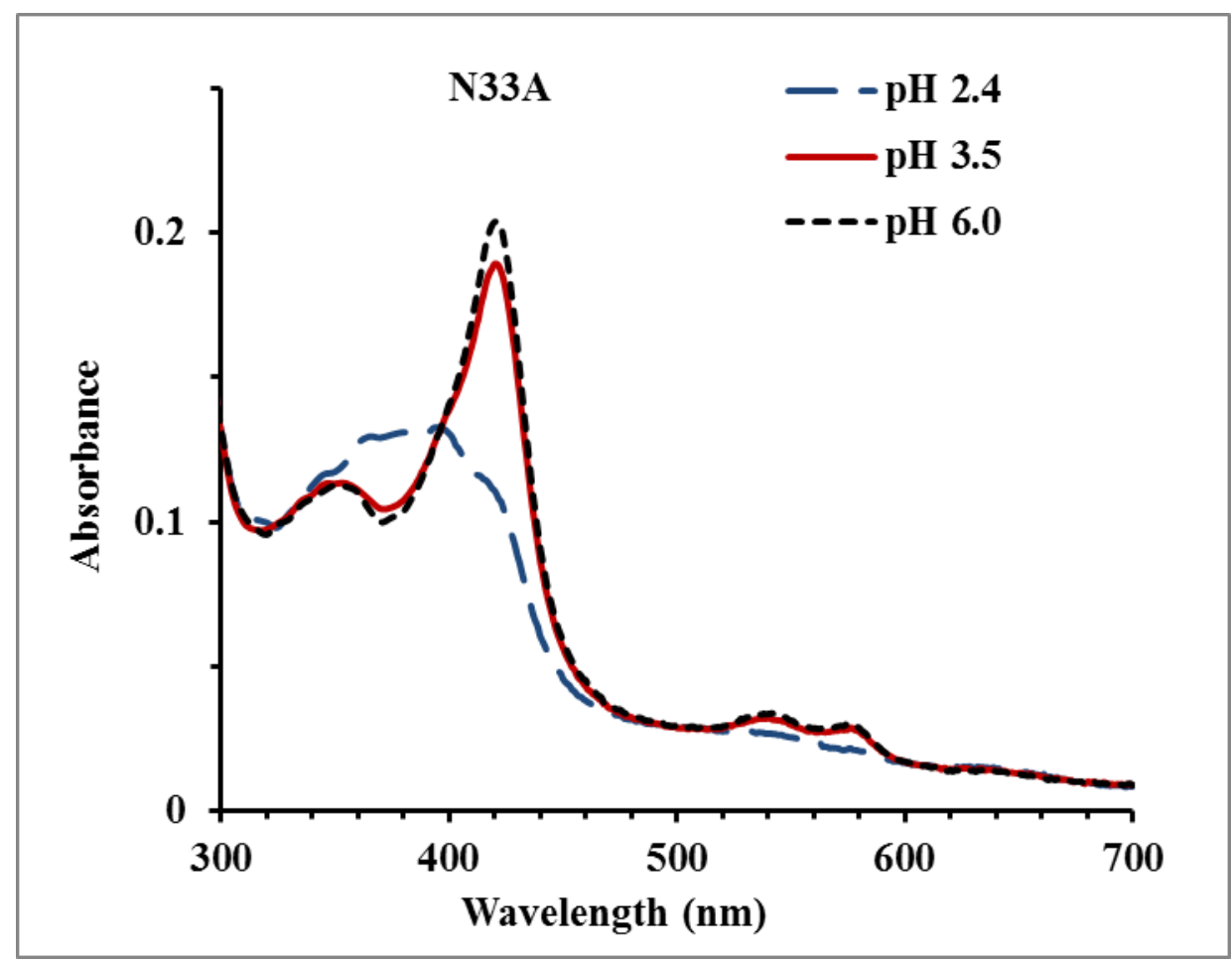

Figure 3.13. UV-Vis absorption spectra of N33A CPO in the acidic $\mathrm{pH}$ range.

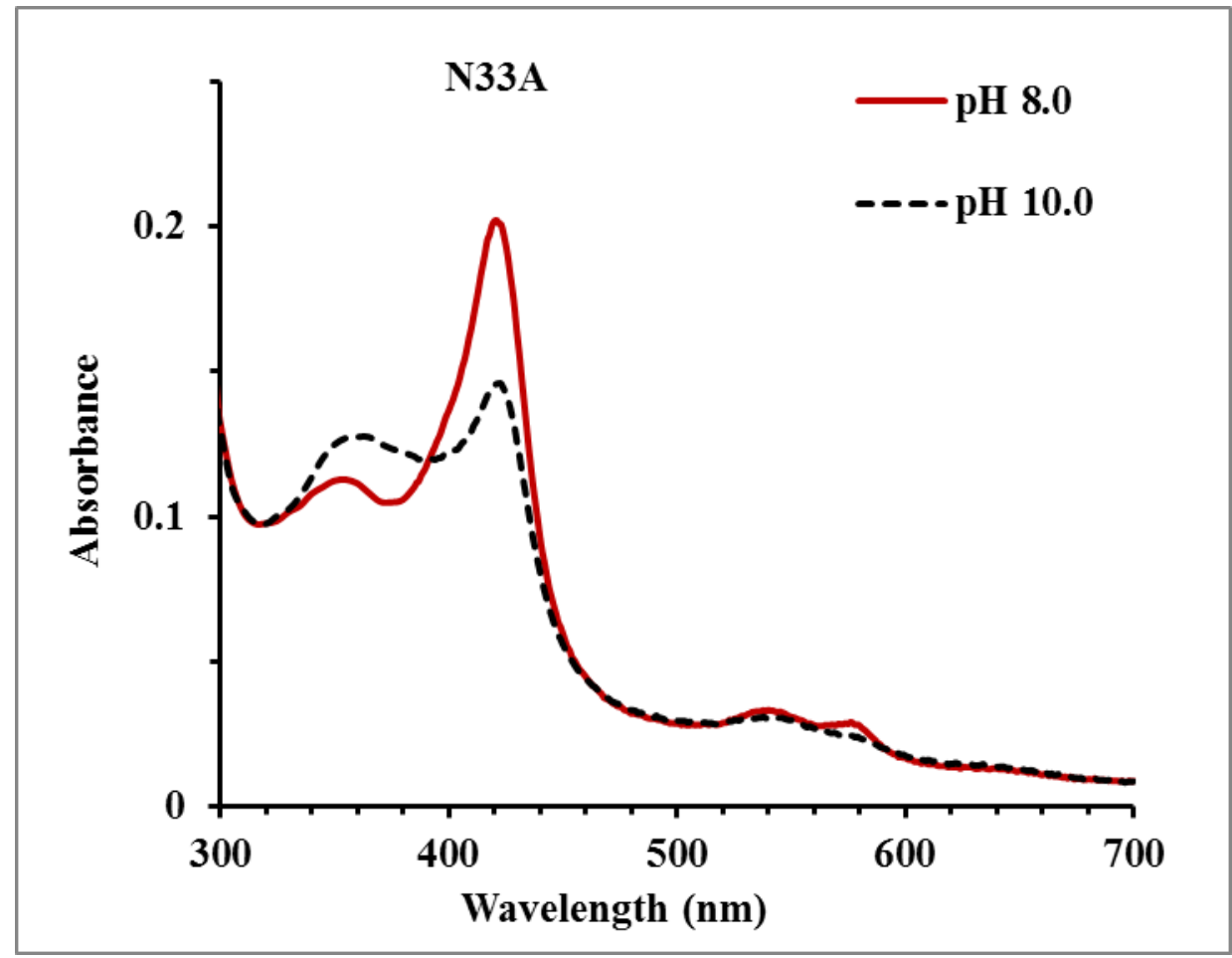

Figure 3.14. UV-Vis absorption spectra of N33A CPO in basic $\mathrm{pH}$. 


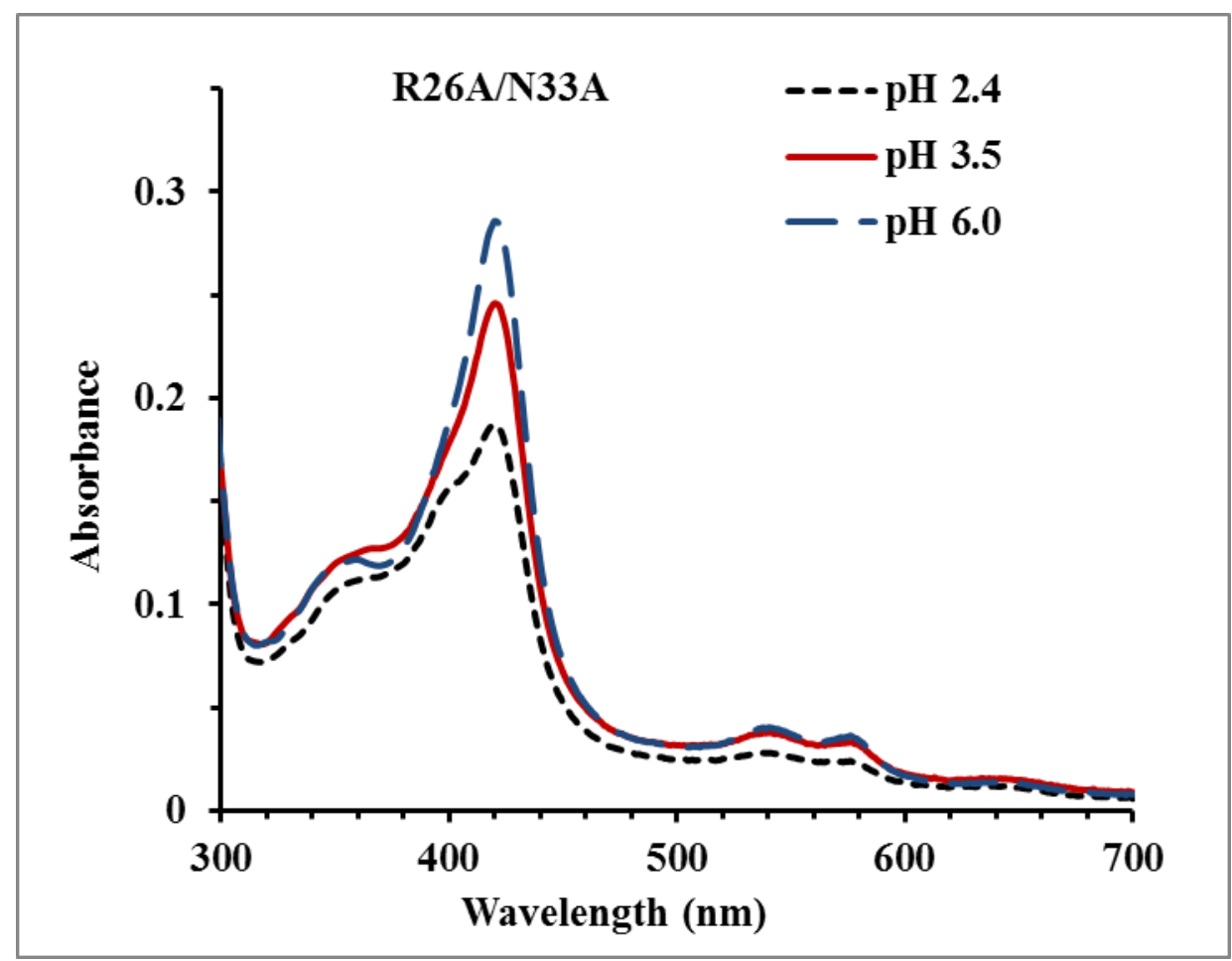

Figure 3.15. UV-Vis absorption spectra of R26A/N33A CPO in the acidic pH range.

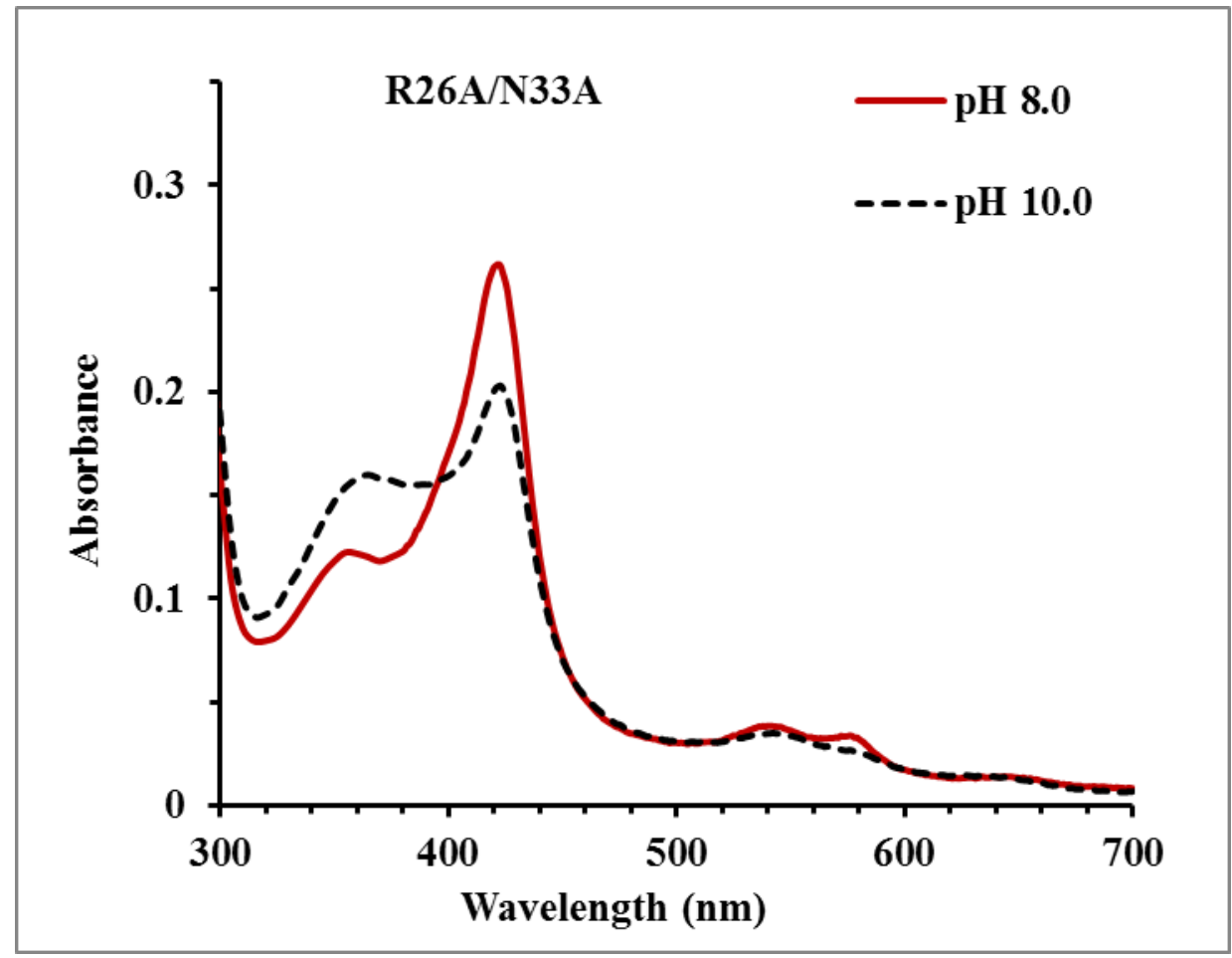

Figure 3.16. UV-Vis absorption spectra of R26A/N33A CPO in basic pH. 


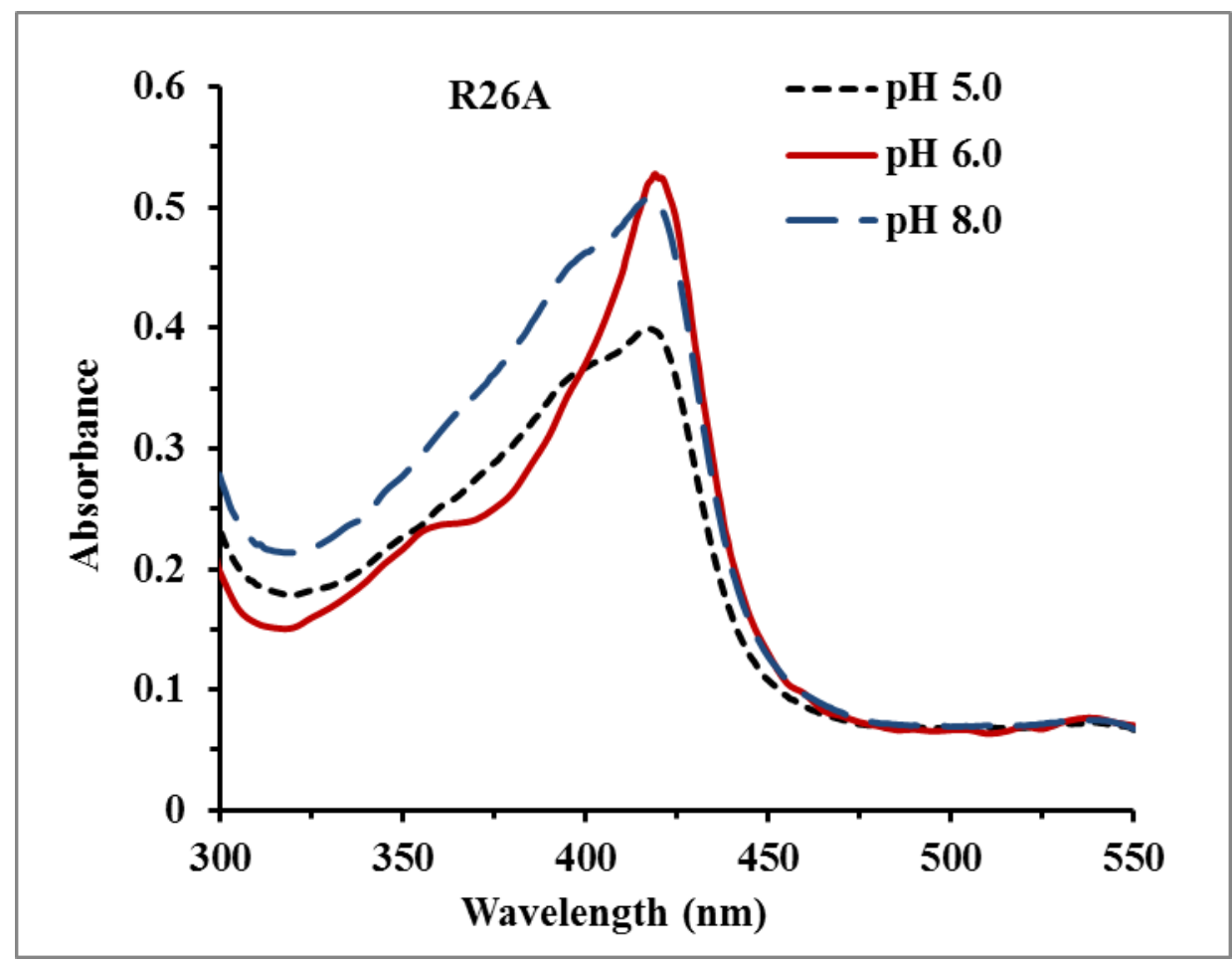

Figure 3.17. UV-Vis absorption spectra of R26A CPO at various $\mathrm{pH}$.

It should be noted that out of all CPO mutants created during my research, R26A had the lowest stability and it was the most difficult to express. Its decreased stability is evident from the spectral study with varying $\mathrm{pH}$ (fig. 3.17). The spectrum of R26A already starts changing at pH 5.0 in the acidic pH range, suggesting a conformational change involving protonation of a residue with a pKa around 5. Similar spectral changes occur at $\mathrm{pH}$ 8.0, indicating a second transition possibly involving deprotonation of another residue with a higher pKa.

\subsubsection{Ligand binding to WT and R26A, N33A, and R26A/N33A CPO}

The ligand-binding properties of CPO mutants can be used to further probe their active site structures. Although WT CPO spectroscopic properties and heme iron coordination are similar to those in P450s, WT CPO possesses a peroxidase-like active site heme environment because its ligand-binding properties resemble those of classical peroxidases 
(174). The size and pKa of exogenous ligands determine how they bind to CPO. Weakly acidic ligands bind in a protonated state and exhibit decreased affinities above their pKa (for example, cyanide, pKa 9.14). Strongly acidic ligands bind in a deprotonated form and exhibit larger variations in affinities especially at more acidic and alkaline $\mathrm{pH}$ (for example, thiocyanate, pKa -1.9). Affinities of neutral ligands do not depend on $\mathrm{pH}$ (for example, $\mathrm{CO})$ (174). In this study, the binding of $\mathrm{CO}$ and $\mathrm{CN}^{-}$to the $\mathrm{WT}$ and mutant $\mathrm{CPO}$ was investigated. Since CO is a neutral ligand whose affinity does not depend on $\mathrm{pH}$, its binding to the ferrous $\mathrm{CPO}$ was monitored at a single $\mathrm{pH}$ of 6.0. In contrast, weakly acidic $\mathrm{CN}^{-}$ should exhibit lower affinity above $\mathrm{pH} 9.14$ and, therefore, its binding to the ferric CPO was investigated at $\mathrm{pH} 6.0$ and $\mathrm{pH} 12.0$.

\subsubsection{CO binding to WT and mutant CPO}

The Soret bands of the WT and mutant CPOs upon reduction with sodium dithionite shift to around $408 \mathrm{~nm}$ (Figs. 3.18-3.21 and Table 3.3).

Table 3.3. Spectral properties of ferrous WT, R26A, N33A, and R26A/N33A CPO and their CO complexes.

\begin{tabular}{lccc}
\hline Protein & $\boldsymbol{\delta}$ band (nm) & Soret (nm) & Q band (nm) \\
\hline WT CPO & -- & 408 & 550 \\
\hline WT-CO & -- & 445 & 550 \\
\hline R26A & -- & 408 & 551 \\
\hline R26A-CO & -- & 444 & 551 \\
\hline N33A & -- & 407 & 551 \\
\hline N33A-CO & 358 & 443 & 551 \\
\hline R26A/N33A & -- & 408 & 550 \\
\hline R26A/N33A-CO & 357 & 444 & 550 \\
\hline
\end{tabular}




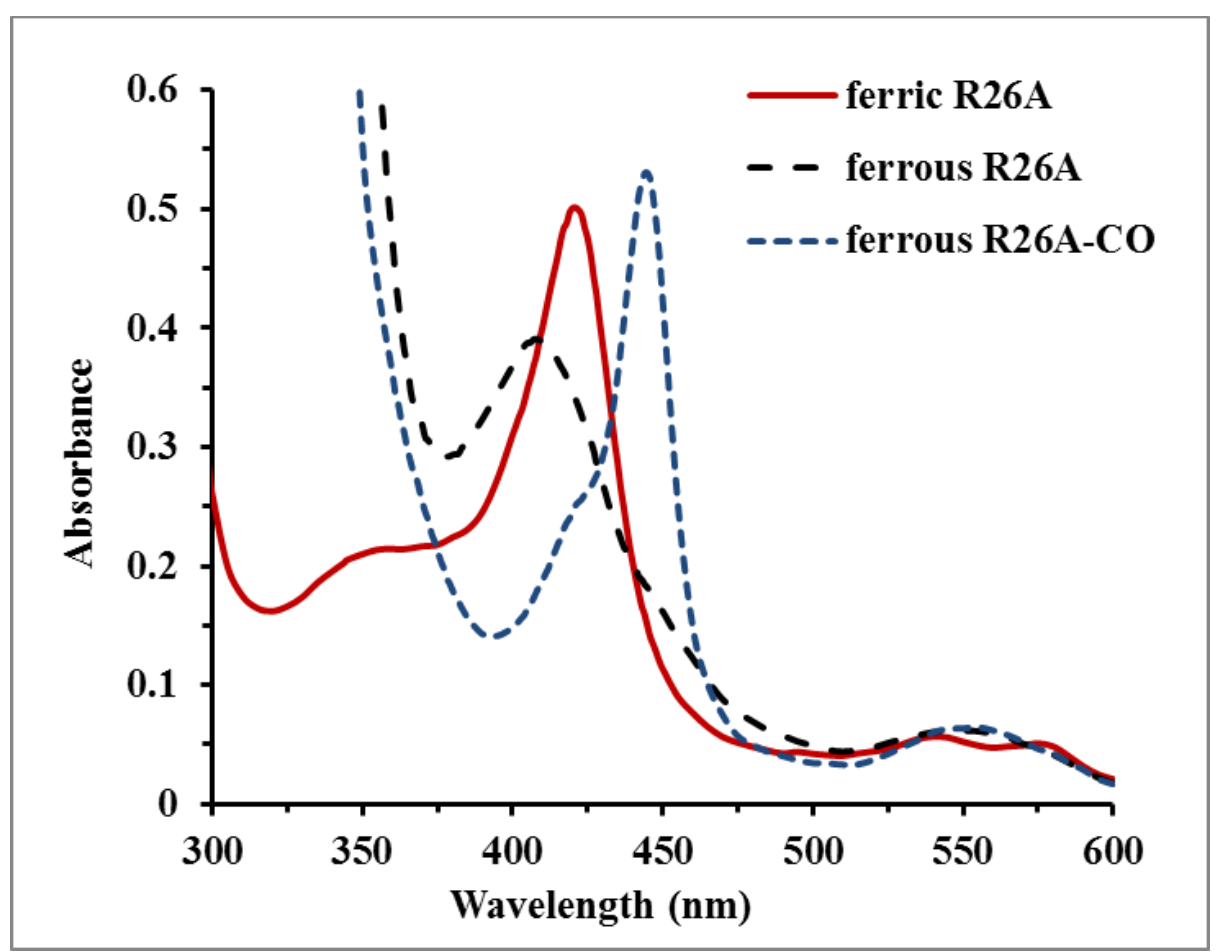

Figure 3.18. UV-Vis absorption spectra of ferric R26A, ferrous R26A, and ferrous R26A-CO complex in phosphate buffer, pH 5.9.

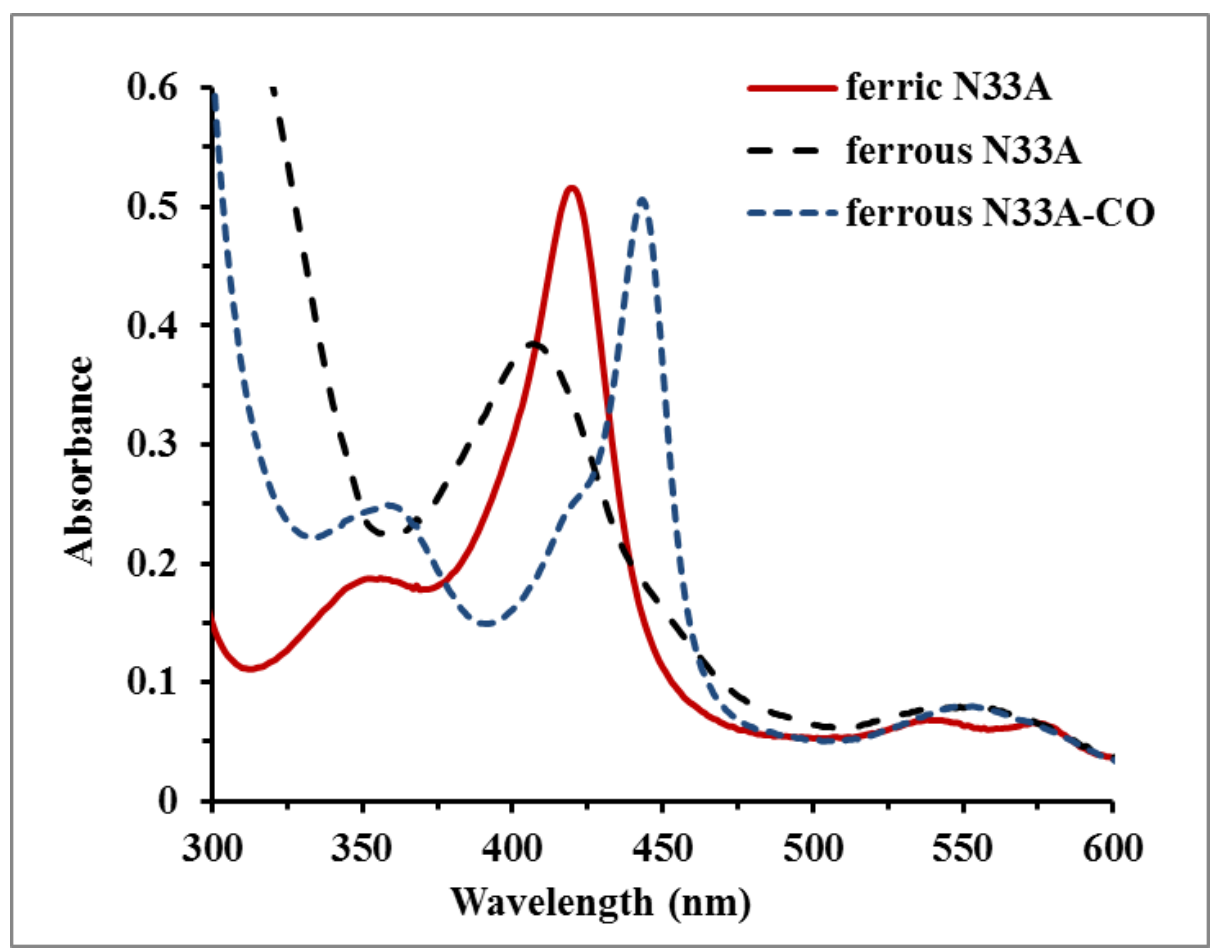

Figure 3.19. UV-Vis absorption spectra of ferric N33A, ferrous N33A, and ferrous N33ACO complex in phosphate buffer, pH 5.9. 


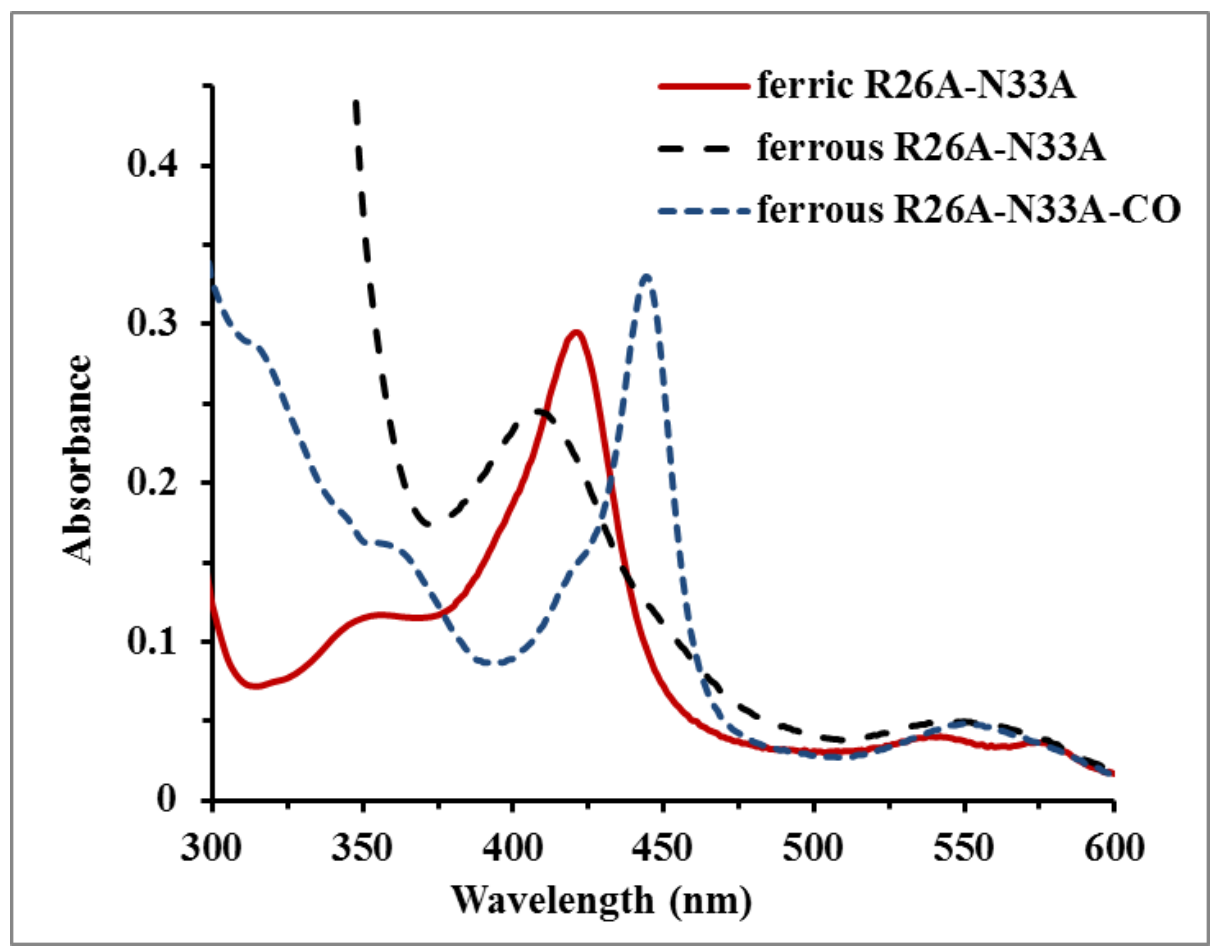

Figure 3.20. UV-Vis absorption spectra of ferric R26A/N33A, ferrous R26A/N33A, and ferrous R26A/N33A-CO complex in phosphate buffer, $\mathrm{pH}$ 5.9.

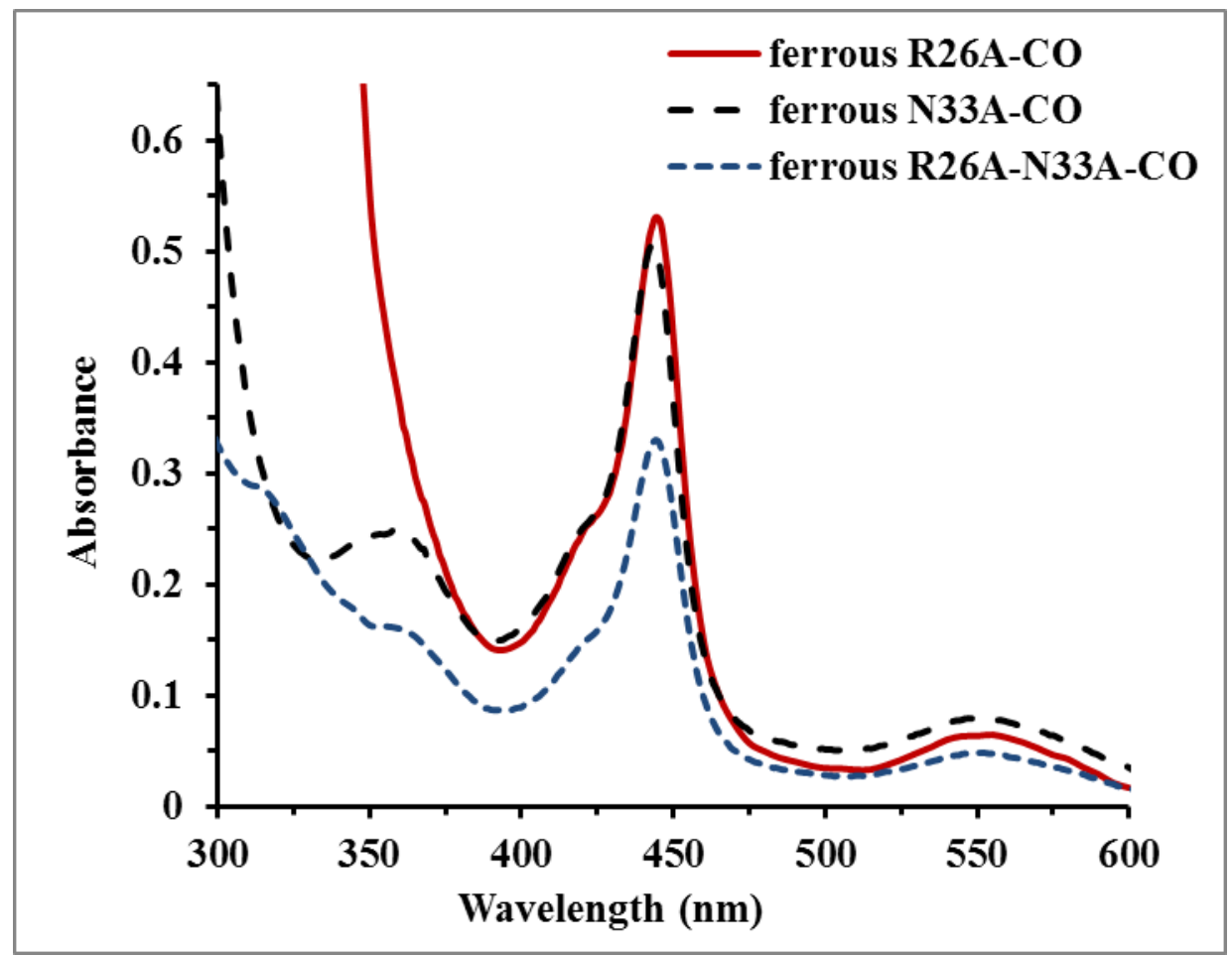

Figure 3.21. Comparison of UV-Vis absorption spectra of ferrous mutant-CO complexes in phosphate buffer, pH 5.9. 
The overall shape of WT, R26A, N33A, and R26A/N33A CPO spectra resemble each other suggesting similar ferrous CPO states in both the WT and mutants. The Soret absorption of the ferrous mutant CPO-CO complexes (443-444 nm) is almost identical to that of the ferrous WT-CO (445 nm) (Fig. 3.21). Interestingly, N33A-CO and R26A/N33A-CO complexes show the delta band at $358 \mathrm{~nm}$ and $357 \mathrm{~nm}$, respectively, with the first mutant having more defined peak (Fig. 3.21). The presence of delta band indicates differences in the electronegative environment of the heme in these two mutants, which can influence the catalytic properties of CPO (discussed in chapter IV).

\subsubsection{Cyanide binding to WT and mutant CPO}

To examine mutant ligand-binding properties further, the UV-Vis spectra of ferric WT and mutant cyanide complexes were obtained at $\mathrm{pH} 6.0$ and $\mathrm{pH} 12.0$ in the presence of $\mathrm{CN}^{-}$excess $(100 \mathrm{mM})$. The spectra were recorded upon addition of cyanide ( $\left.0 \mathrm{~min}\right)$ and after incubation of the sample for 1 hour at room temperature (60 min) (Figs. 3.22-3.25). First, I will discuss results obtained at acidic pH. The absorption spectra of the ferric WTCN and mutant-CN resemble each other, exhibiting delta, Soret, and alpha bands. The initial Soret position in the mutant-CN spectra is similar to that of the WT-CN (Table 3.4). However, after a 1-hour incubation, mutant Soret bands have shifted to slightly different locations with N33A-CN Soret blue-shifted the most $(10 \mathrm{~nm})$ while R26A and R26A/N33A Soret bands blue-shifted only $4 \mathrm{~nm}$ and $2 \mathrm{~nm}$, respectively. The spectrum of the ferric WT$\mathrm{CN}$ remained the same after 1 hour, indicating stronger $\mathrm{CN}^{-}$binding to the WT. To address the question of whether $\mathrm{CN}^{-}$affinity is higher for the WT CPO than for the mutants, both WT and mutant CPOs were titrated with potassium cyanide at $\mathrm{pH} 6.0$ to determine cyanide dissociation constant. 


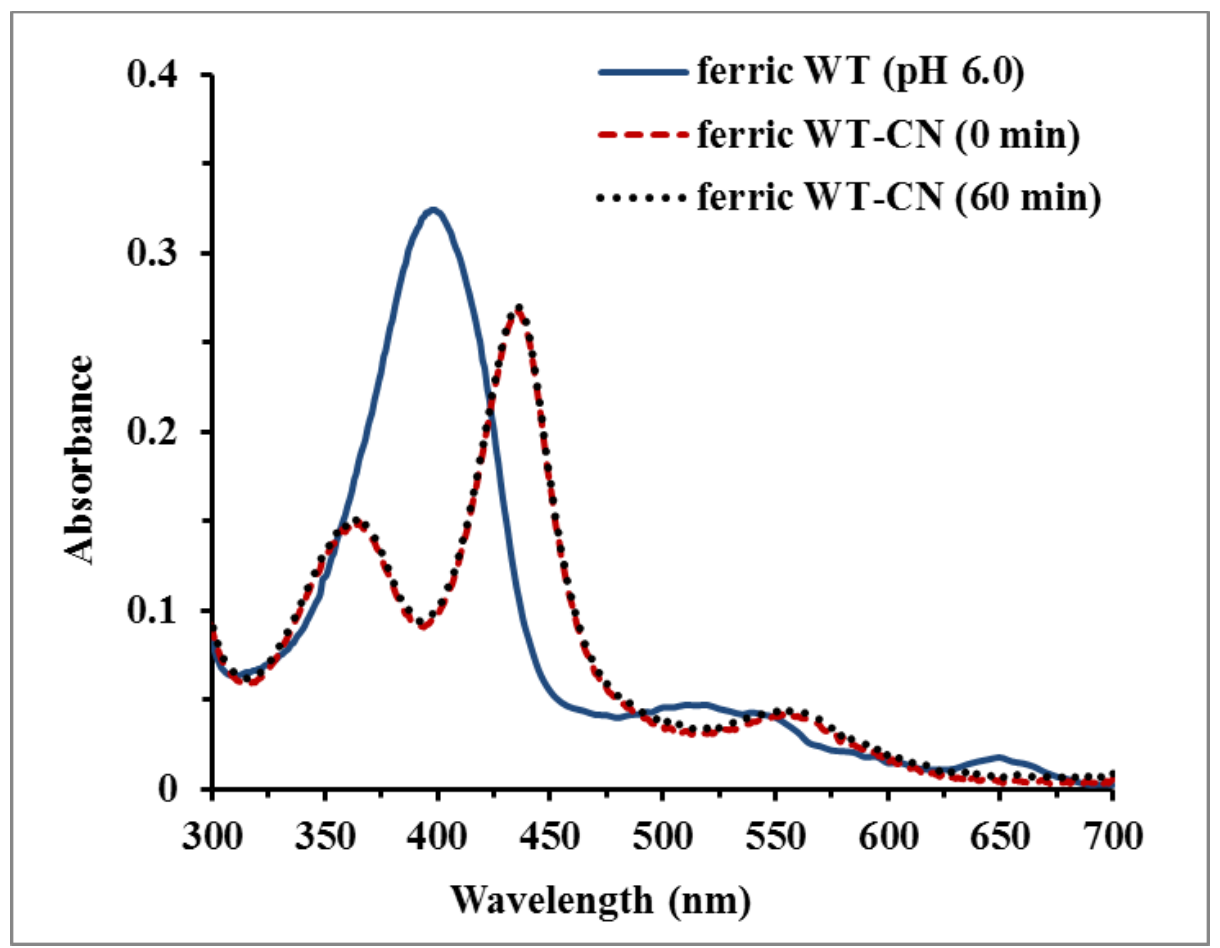

Figure 3.22. Cyanide (100 mM) binding to WT CPO in phosphate buffer, pH 6.0.

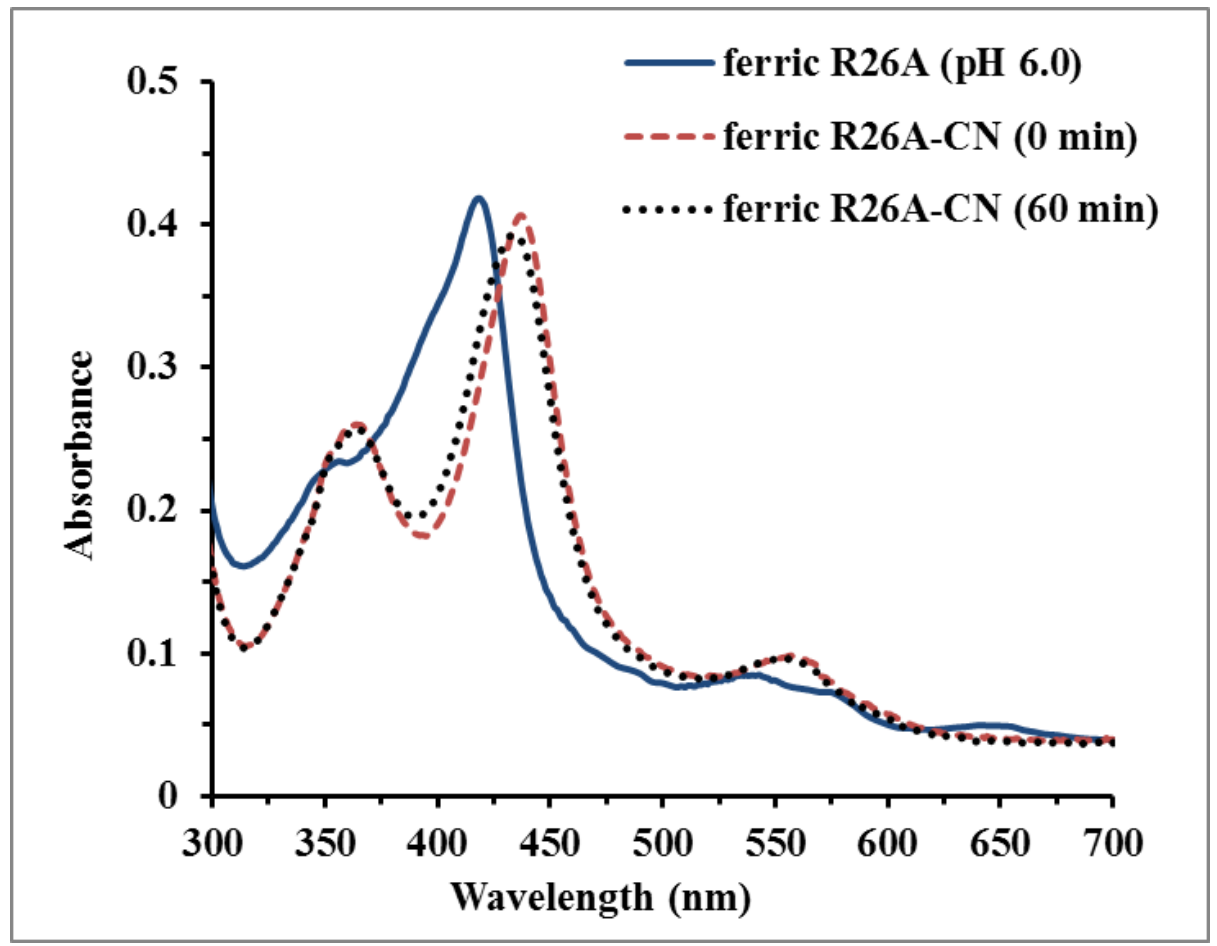

Figure 3.23. Cyanide (100 mM) binding to R26A CPO in phosphate buffer, pH 6.0. 


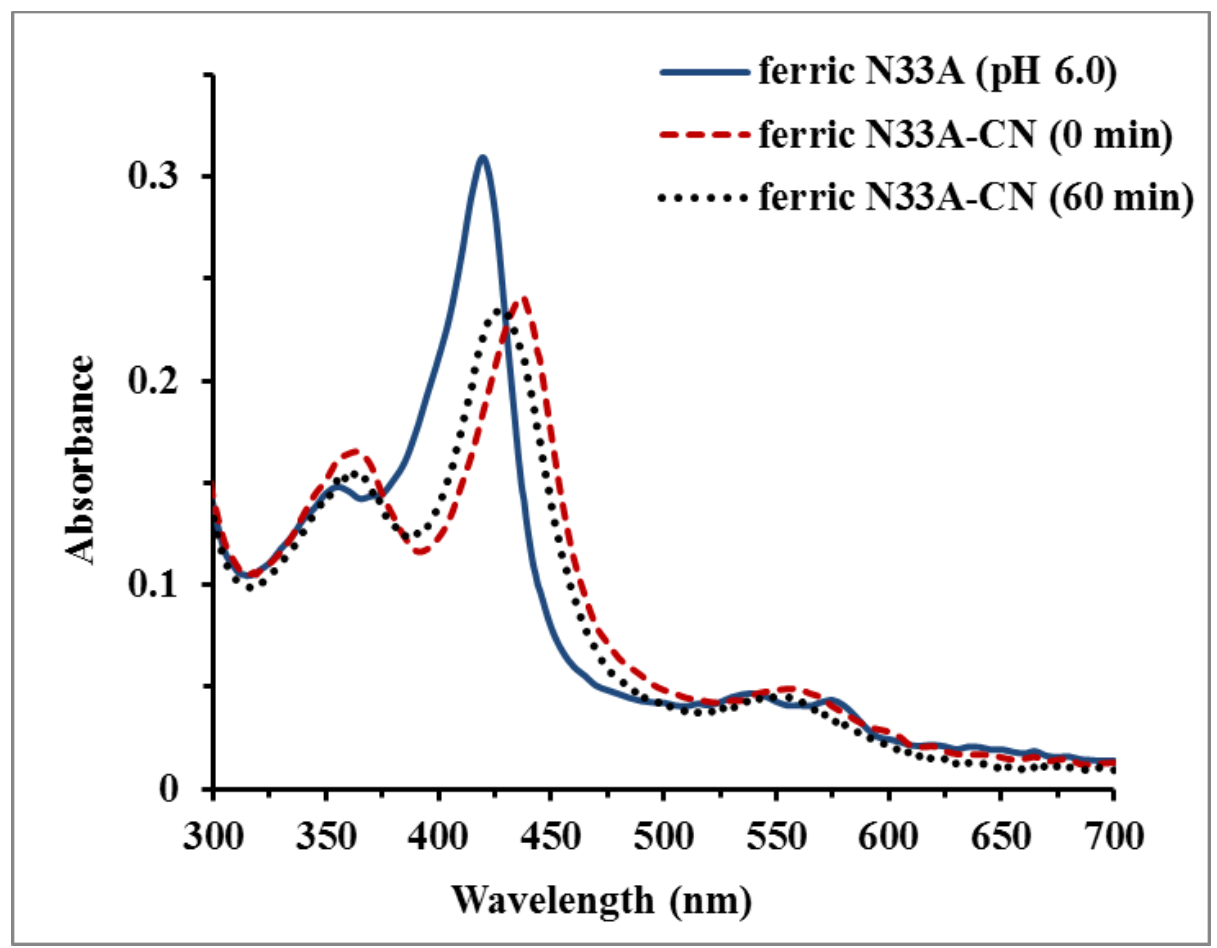

Figure 3.24. Cyanide (100 mM) binding to N33A CPO in phosphate buffer, pH 6.0.

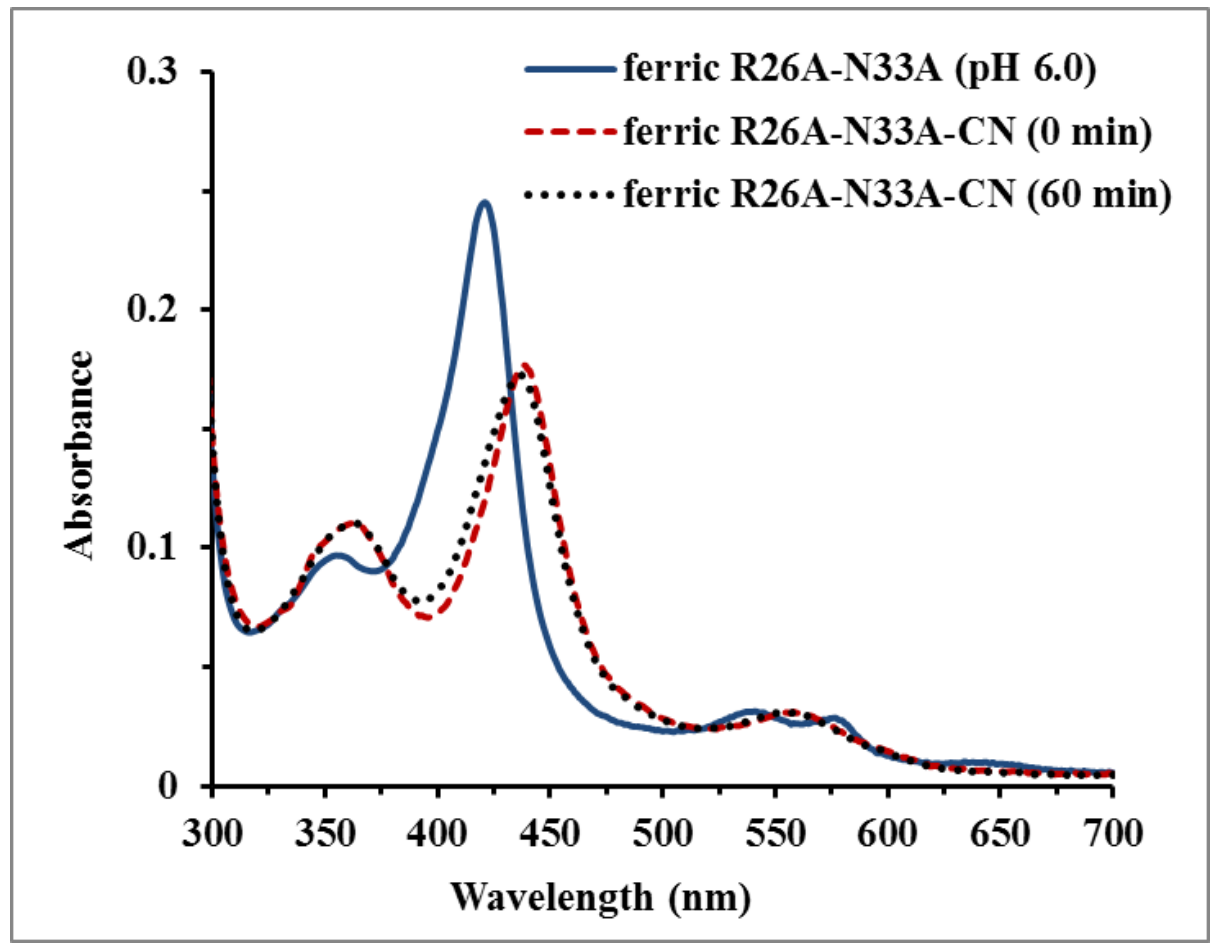

Figure 3.25. Cyanide (100 mM) binding to R26A/N33A CPO in phosphate buffer, pH 6.0. 
Since the pre-requisite for using Eq. 3.1 to determine Kd is titrations with excess ligand, 1-10 $\mathrm{mM}$ (for mutants) or 2-10 $\mathrm{mM}$ (for WT) cyanide was added to protein solutions (3-8 $\mu \mathrm{M}$ ) in titration studies. Upon addition of $2 \mathrm{mM} \mathrm{CN}^{-}$to the WT CPO (red spectrum), the Soret band shifted from $398 \mathrm{~nm}$ to $439 \mathrm{~nm}$ (Fig. 3.26, A). Upon gradual increase of $\mathrm{CN}^{-}$concentration, the Soret peak has remained at $439 \mathrm{~nm}$ while absorption has slightly increased. The shapes of other WT-CN spectra $\left(4-10 \mathrm{mM} \mathrm{CN}^{-}\right)$are essentially identical, and there is no isosbestic point formed by these spectra indicating the presence of a single species in the solution at higher ligand concentrations. The spectrum of WT $\mathrm{CPO}$ after addition of $2 \mathrm{mM} \mathrm{CN}^{-}$(red) has a slightly different shape, and it possesses an isosbestic point ( $427 \mathrm{~nm}$ ) with spectra taken at higher $\mathrm{CN}^{-}$concentrations. This isosbestic point can be explained by the presence of the equilibrium between uncomplexed WT and WT-CN upon initial addition of $\mathrm{CN}^{-}$. Once the concentration of the ligand is increased, the equilibrium shifts to WT-CN side, and no further changes in the WT-CN spectra are observed. Since the shift of the Soret band is observed upon addition of $2 \mathrm{mM} \mathrm{CN}^{-}$, WT has been titrated with 10-800 $\mu \mathrm{M}$ cyanide to observe gradual changes in the WT spectrum. However, at these cyanide concentrations the protein to ligand ratio is smaller with the highest being approximately 1:100, which introduces larger error when using Eq. 3.1 for Kd determination.

Upon addition of lower concentrations of $\mathrm{CN}^{-}(10-800 \mu \mathrm{M})$ to the WT CPO, the intensity of the Soret band at $398 \mathrm{~nm}$ has gradually decreased while a new peak has formed at $439 \mathrm{~nm}$, indicating formation of WT-CN complex (Fig. 3.26, B). The isosbestic point at $427 \mathrm{~nm}$ is consistent with the presence of two species in the solution, uncomplexed WT CPO and WT bound to cyanide. The Kd value calculated in this study, $130.0 \pm 6.5 \mu \mathrm{M}$, is 
close to the Kd reported previously for CPO-CN at pH 6.0, 95 $\pm 10 \mu \mathrm{M}$ (243). The difference in values may arise from temperature variations during titrations and the fact that the protein to ligand ratio was not high enough as required for calculations using Eq. 3.1. However, addition of higher concentrations of the cyanide did not produce large enough change in the spectrum of the WT that is needed for Kd determination. Thus, Kd result here should be treated as an estimate.

When $1 \mathrm{mM} \mathrm{CN}^{-}$is added to the mutants, the intensity of the Soret peak decreases and it red-shifts to $421 \mathrm{~nm}$ for R26A and N33A mutants (Figs. 3.27 and 3.28) and to 422 $\mathrm{nm}$ for R26A/N33A (Fig. 3.29). However, when $\mathrm{CN}^{-}$concentration is increased, the Soret band moves to $422 \mathrm{~nm}, 425 \mathrm{~nm}$, and $430 \mathrm{~nm}$ for R26A, N33A, and R26A/N33A, respectively, while band intensities decrease further. The presence of the isosbestic point in all mutant spectra is consistent with the equilibrium between uncomplexed CPO and CPO-CN species. Higher $\mathrm{CN}^{-}$concentrations (mM range) required to observe spectral changes indicate that cyanide has lower affinity for the mutants. The data for $\mathrm{Kd}$ determination is plotted in Fig. 3.30. Calculated Kd values for R26A, N33A, and R26A/N33A are $3.6 \mathrm{mM}, 10.5 \pm 1.0 \mathrm{mM}$, and $3.8 \pm 0.4 \mathrm{mM}$, respectively, which confirms that $\mathrm{CN}^{-}$has lower affinity for the mutants. The Kd result for R26A mutant does not include the standard deviation and should be treated with caution since only one trial was performed because of the low yield of this mutant.

Previous studies on ligand binding to CPO revealed that cyanide diffuses into the distal heme pocket in a protonated form, HCN, and loses its proton upon binding to the heme iron $(174,220)$. Since WT CPO has no distal ligand, HCN can bind to the heme more readily. In contrast, R26A, N33A, and R26A/N33A CPO have low-spin ferric heme and 
likely possess water or another amino acid as a distal ligand that competes with $\mathrm{CN}^{-}$for binding to the heme, which would explain lower $\mathrm{CN}^{-}$affinity for the mutants.

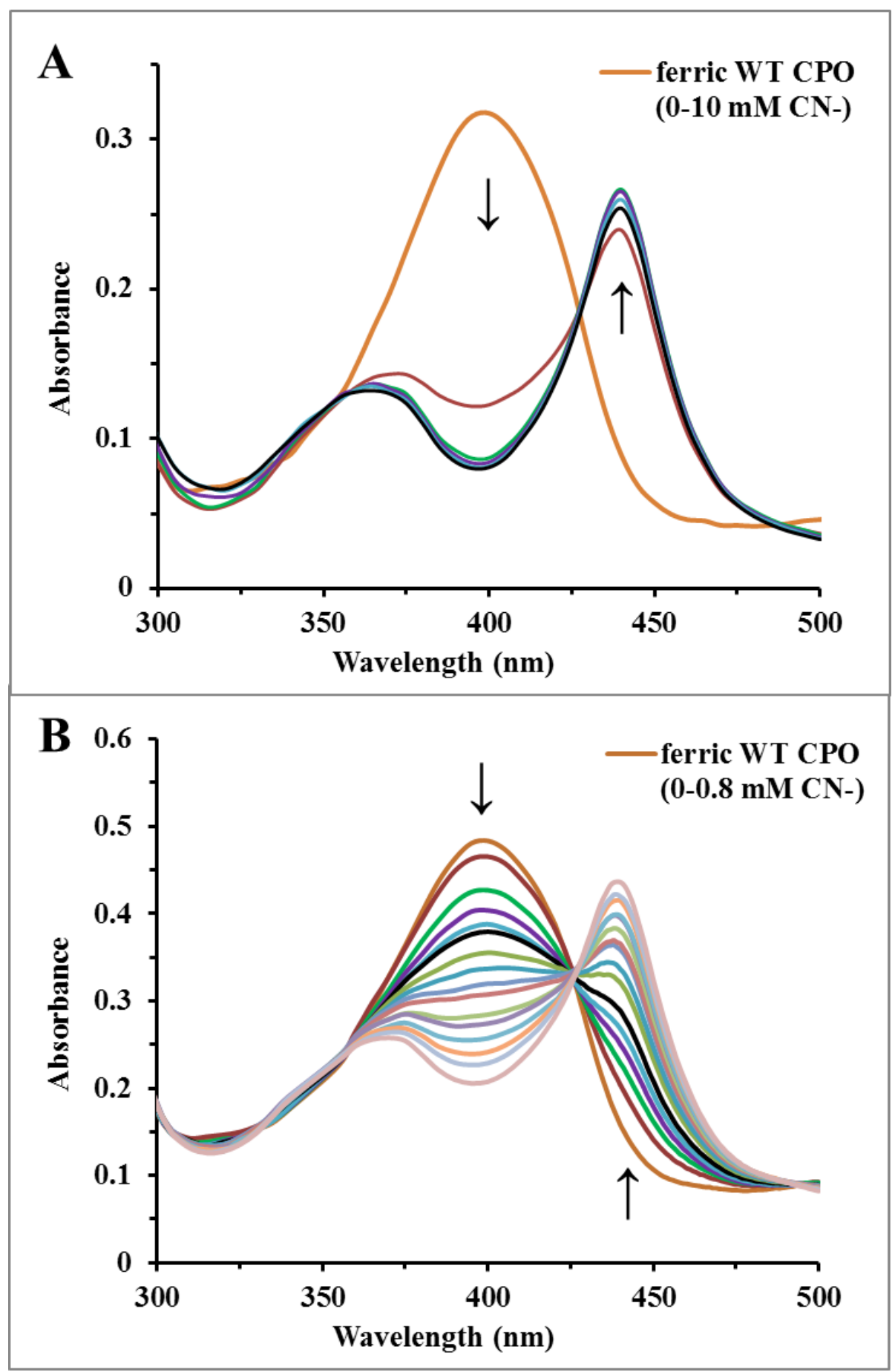

Figure 3.26. Titration of WT CPO with cyanide in phosphate buffer, $\mathrm{pH}$ 6.0. A. 2-10 mM CN-. B. 10-800 $\mu \mathrm{M}$ CN-. 


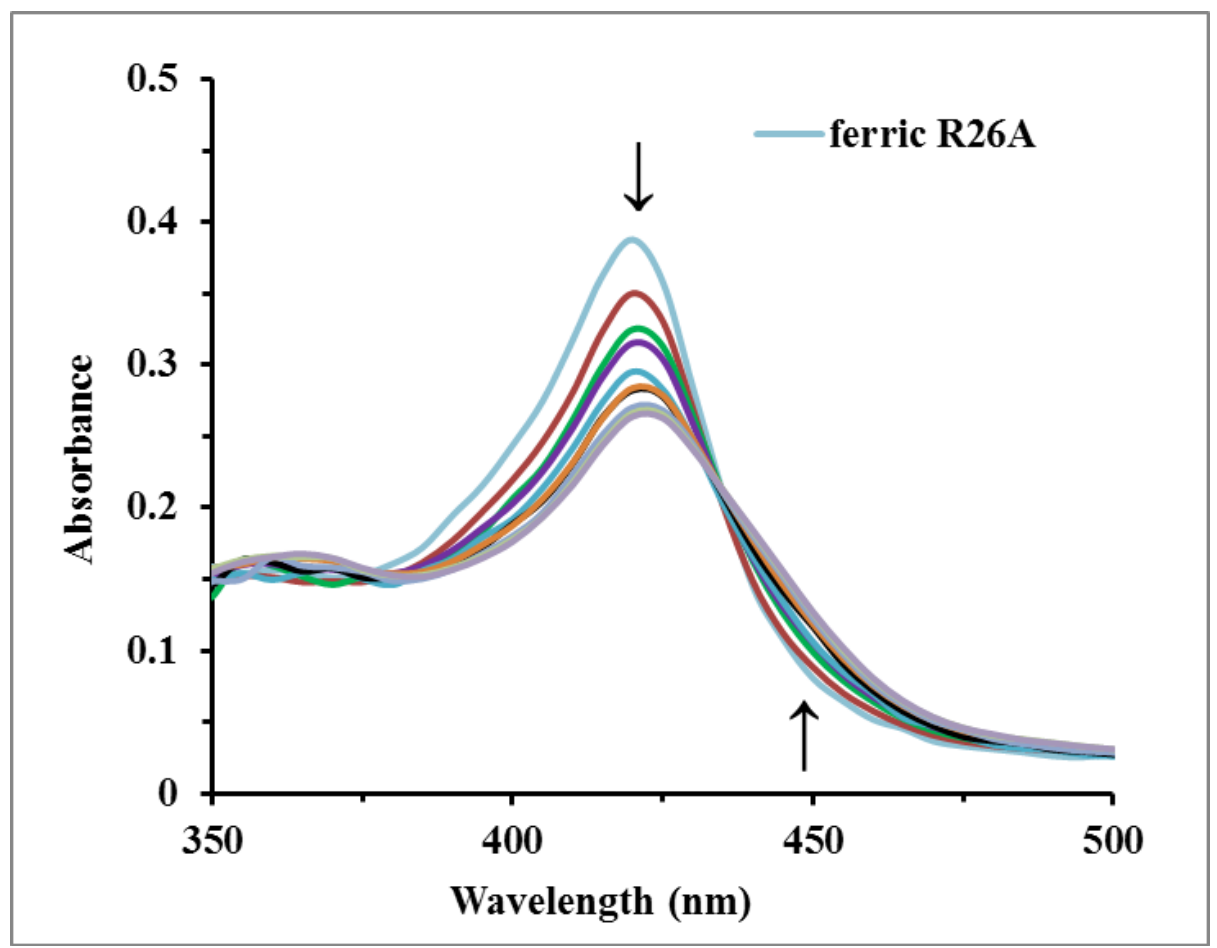

Figure 3.27. Titration of R26A CPO with $\mathrm{CN}^{-}(1-10 \mathrm{mM})$ in phosphate buffer, pH 6.0.

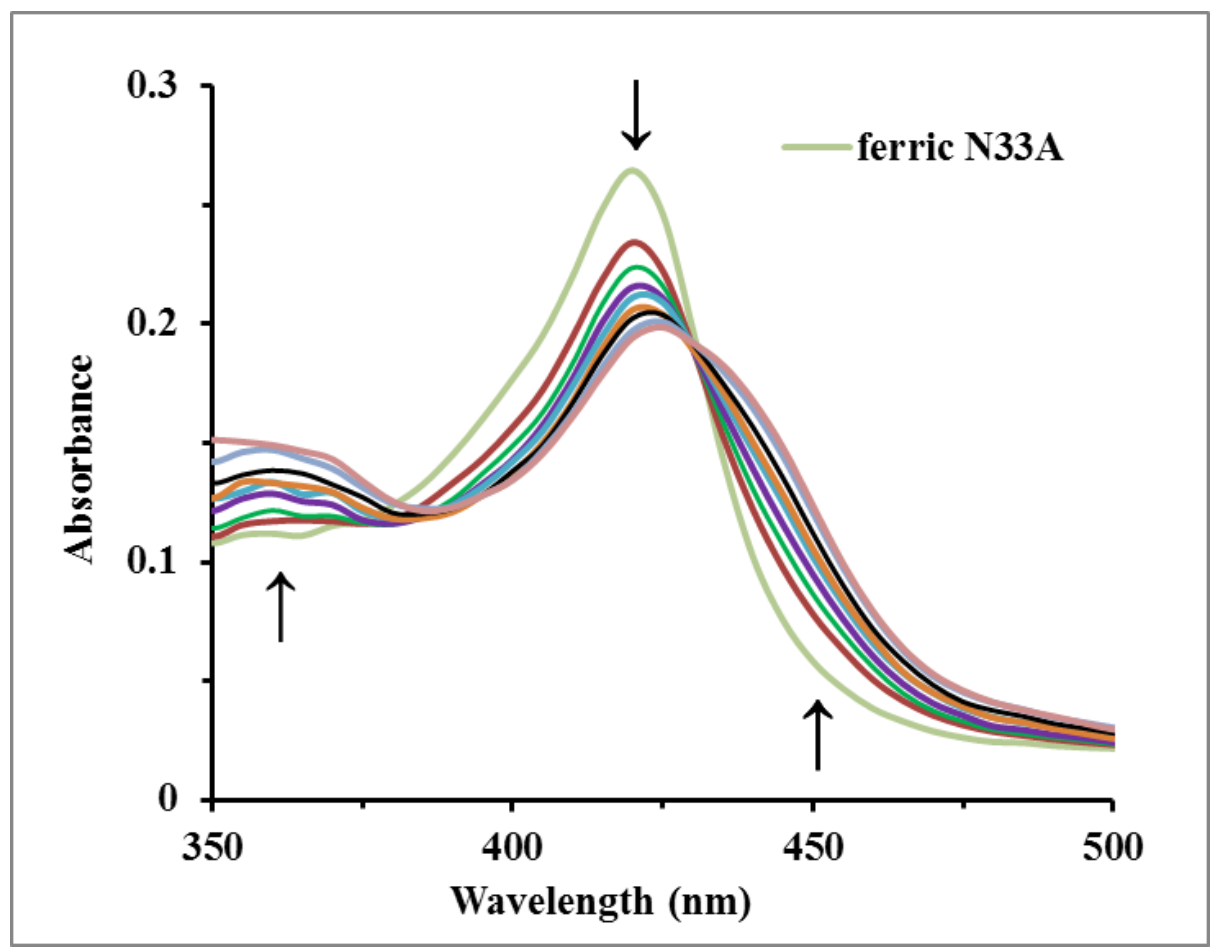

Figure 3.28. Titration of N33A CPO with $\mathrm{CN}^{-}(1-10 \mathrm{mM})$ in phosphate buffer, pH 6.0. 


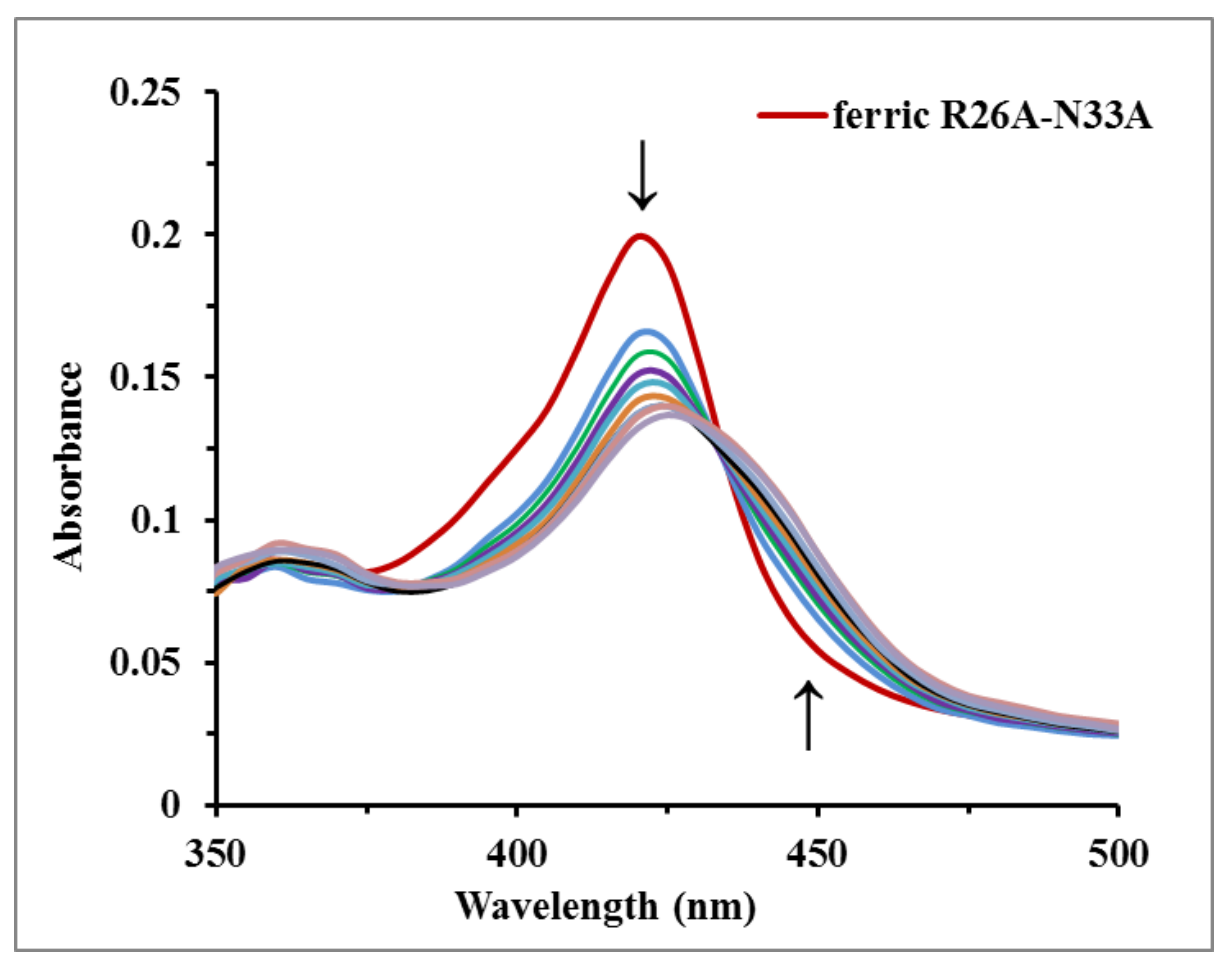

Figure 3.29. Titration of R26A/N33A with $\mathrm{CN}^{-}(1-10 \mathrm{mM})$ in phosphate buffer, $\mathrm{pH}$ 6.0.

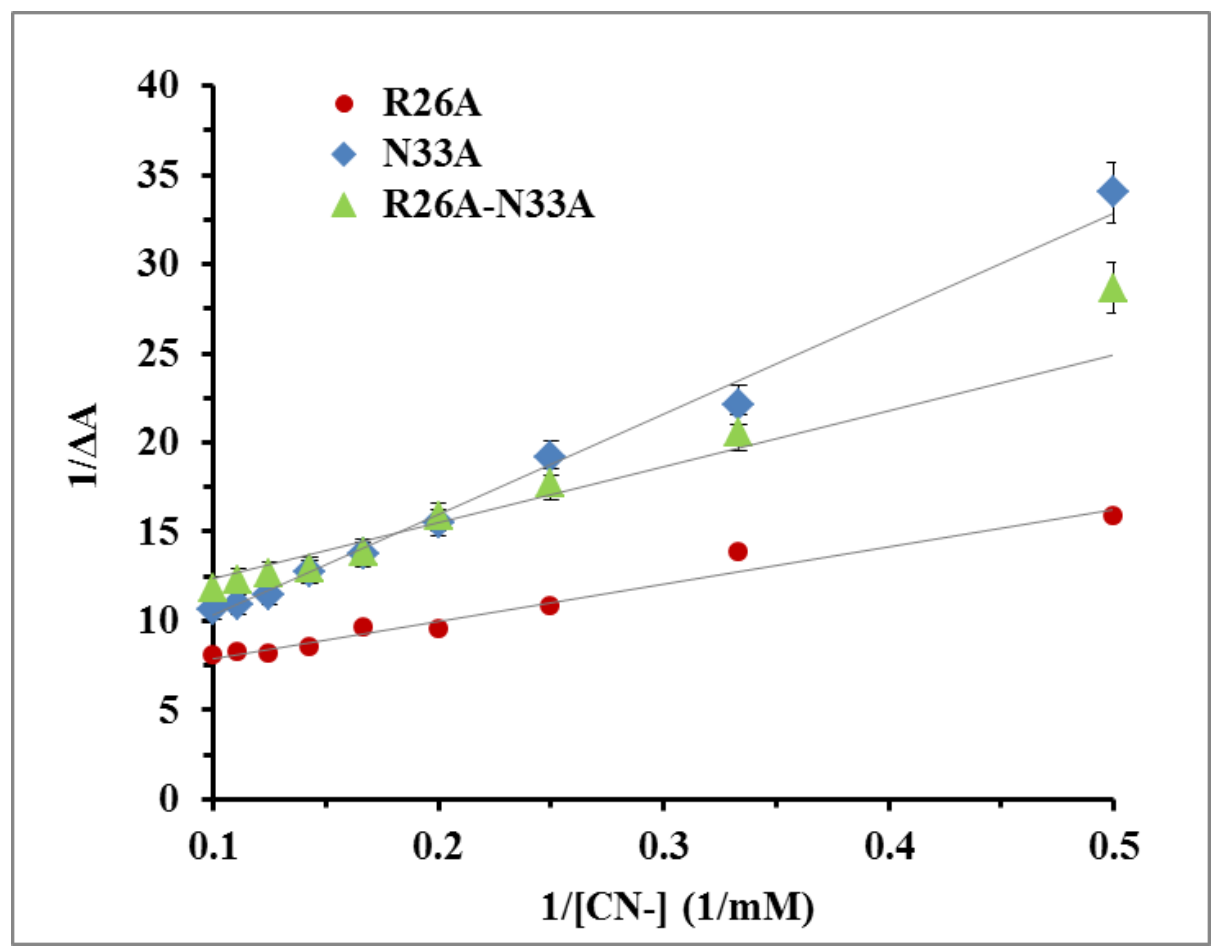

Figure 3.30. $\mathrm{CN}^{-} \mathrm{Kd}$ determination for the mutant $\mathrm{CPO}$ (Only 1 trial for R26A was performed because of the limited amount of the protein, hence there are no error bars). 
The Hill plot (Fig. 3.31) (240) was used to explore the binding stoichiometry of $\mathrm{CN}^{-}$ to mutant CPOs. The slopes of all three graphs are very close to 1 confirming that $\mathrm{CN}^{-}$binding to the R26A, N33A, and R26A/N33A mutants follows the same stoichiometry as in binding to the WT: one molecule of cyanide binds to one molecule of CPO.

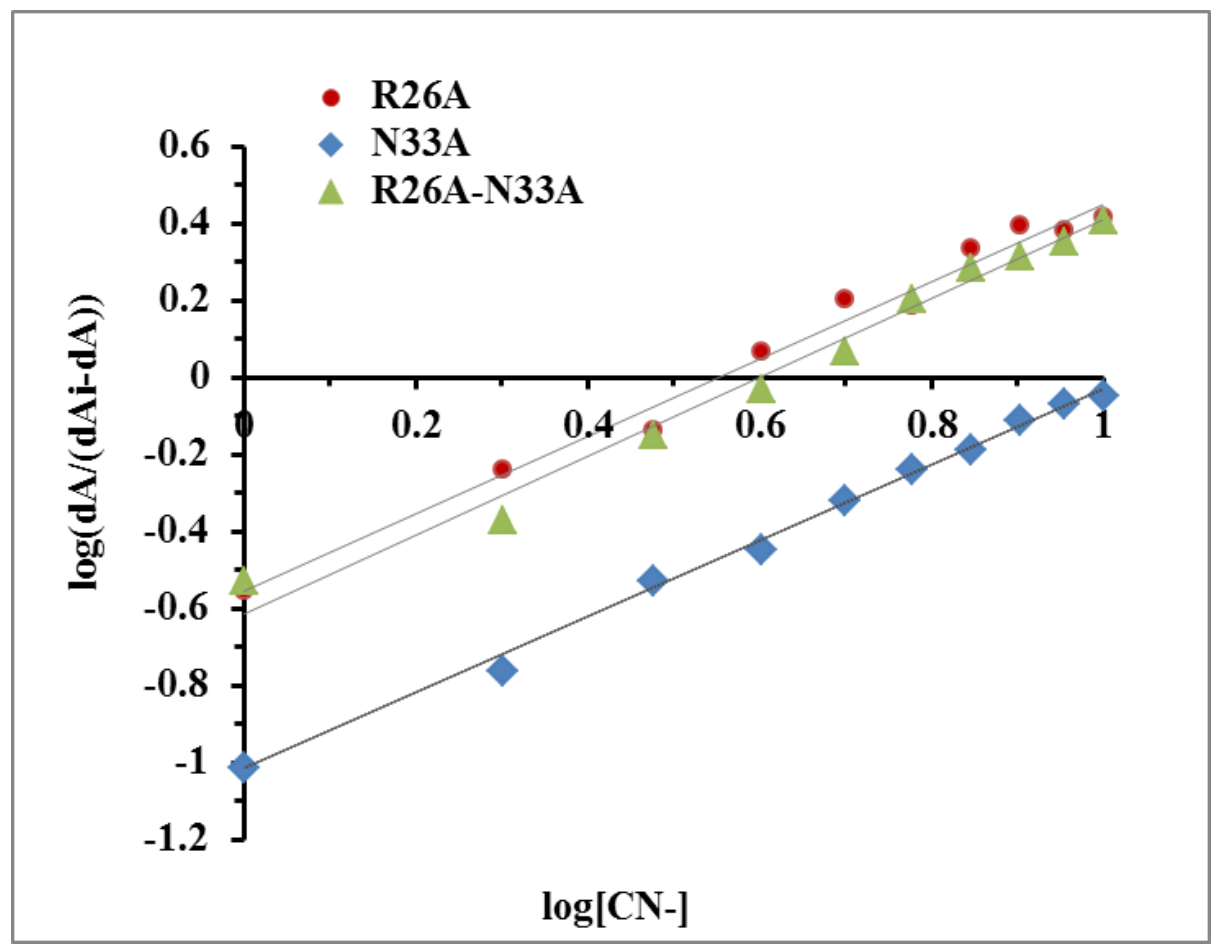

Figure 3.31. The Hill plot (determination of mutant CPO-CN binding stoichiometry). The slopes of R26A, N33A, and R26A-N33A plots are 1.0048, 0.9943, and 1.0249, respectively.

Cyanide (100 mM) binding was also performed at pH 12.0 to probe its ability to bind to mutant CPO in deprotonated form (pKa 9.14). The WT spectrum at pH 12.0 is consistent with the second alkaline transition occurring near $\mathrm{pH}$ 11.0, reported previously for CPO (Fig. 3.32) (220). Alkaline transitions at $\mathrm{pH} 12.0$ have been also observed in N33A and R26A/N33A CPOs (Fig. 3.33 and 3.34). The $\mathrm{CN}^{-}$binding to R26A at alkaline $\mathrm{pH}$ has not been performed because of the low yield of R26A. Spectral properties of CPO and cyanide complexes are summarized in Table 3.4. 


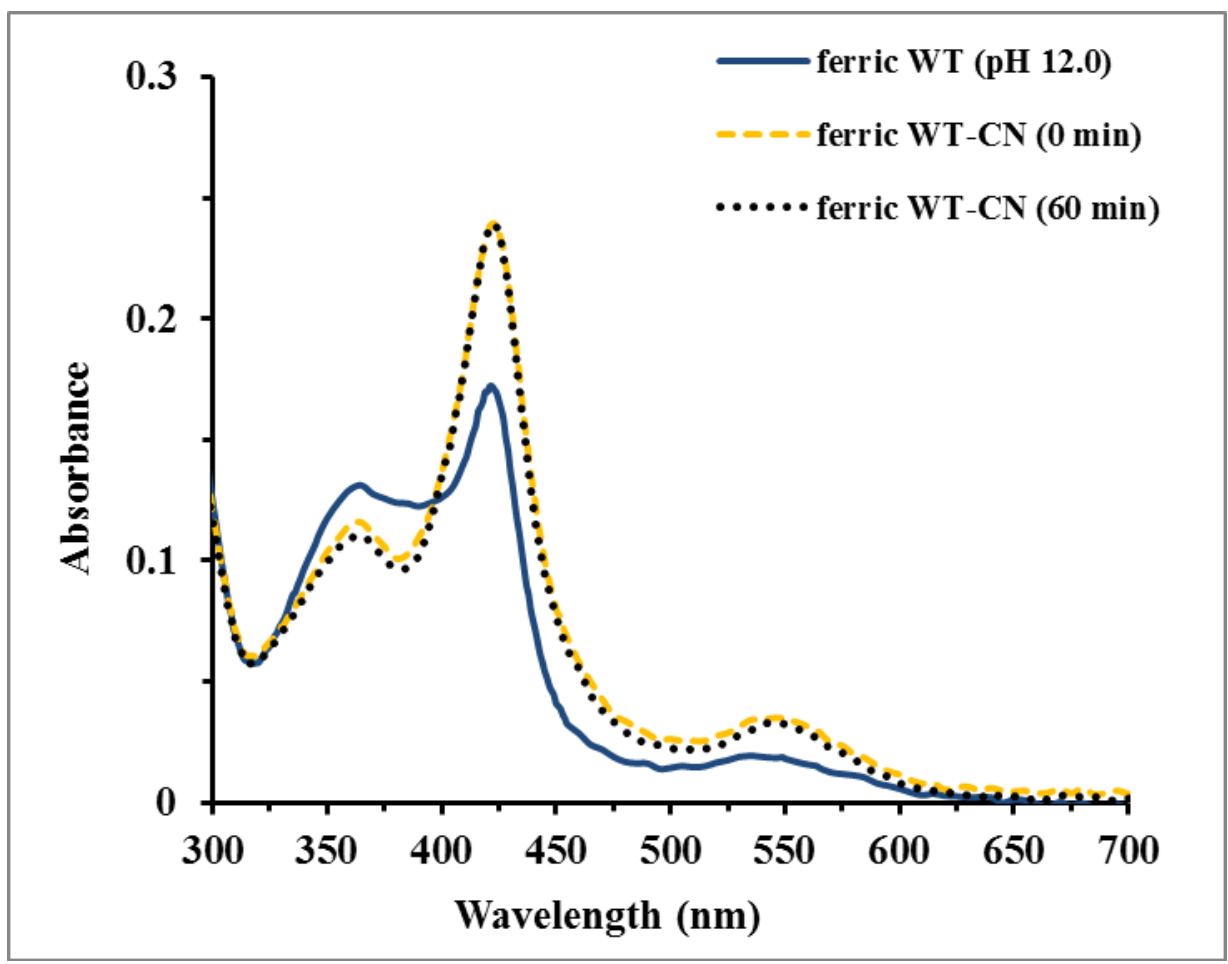

Figure 3.32. Cyanide (100 mM) binding to WT CPO in phosphate buffer, pH 12.0.

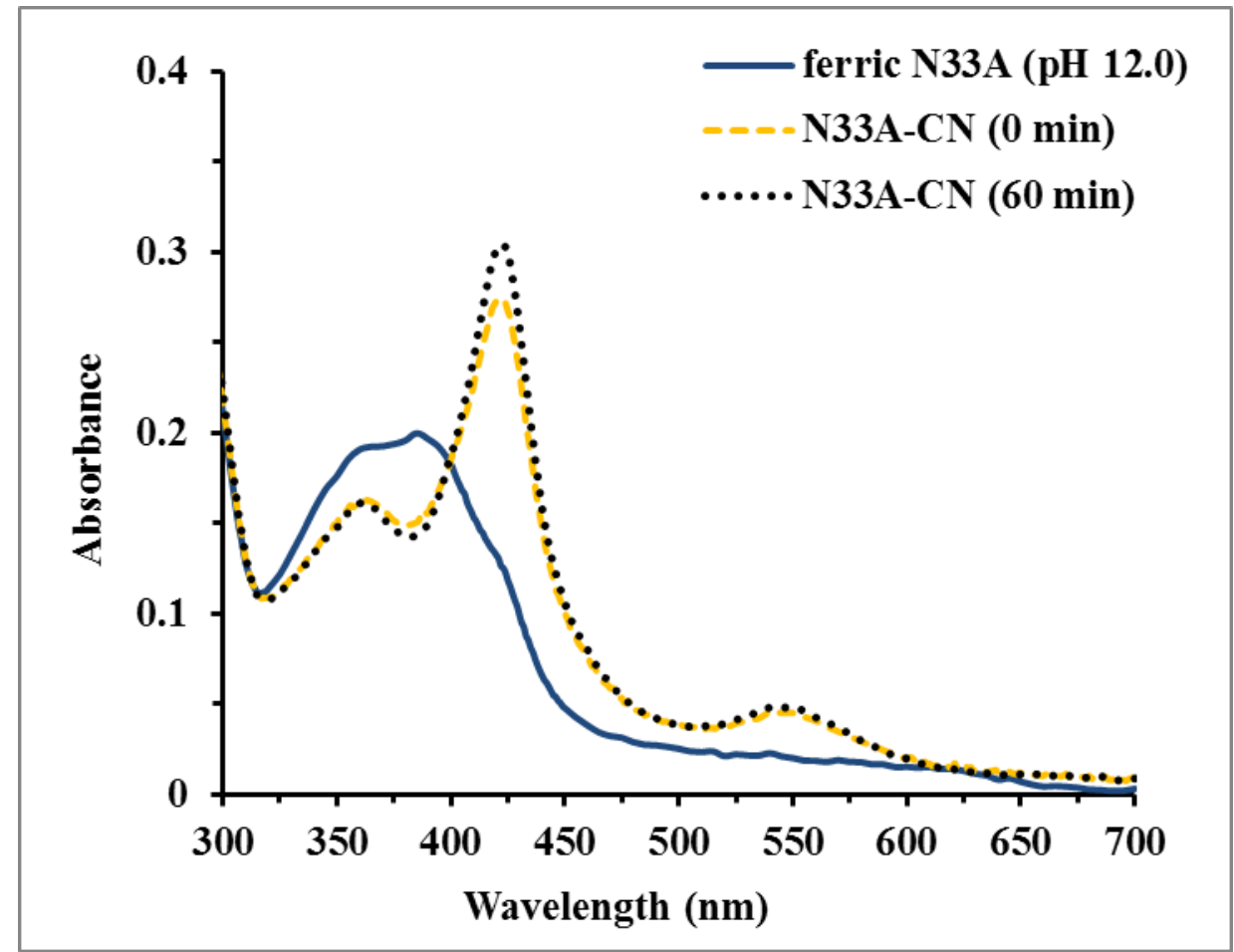

Figure 3.33. Cyanide (100 mM) binding to N33A CPO in phosphate buffer, $\mathrm{pH} 12.0$. 


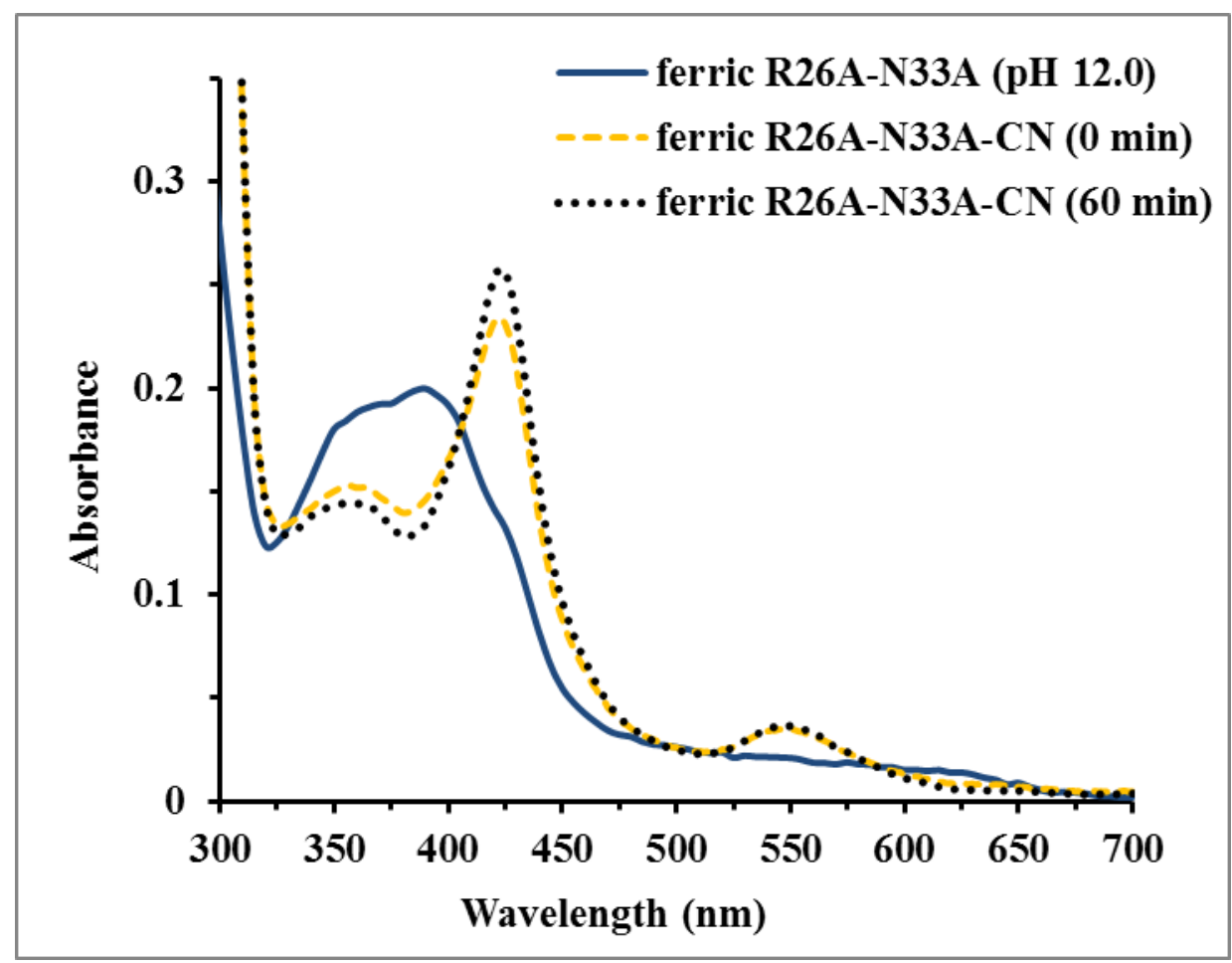

Figure 3.34. Cyanide (100 mM) binding to R26A/N33A in phosphate buffer, pH 12.0.

When $100 \mathrm{mM} \mathrm{CN}^{-}$is added to the WT, N33A, and R26A/N33A, the spectra of all three complexes look essentially the same, with the Soret band at $422 \mathrm{~nm}$, and do not change after 1-hour incubation suggesting that these cyanide derivatives are different from those formed at $\mathrm{pH}$ 6.0. This result is expected since the native forms of the WT and mutants at $\mathrm{pH} 12.0$ are different from those at $\mathrm{pH}$ 6.0.

The mechanism of $\mathrm{CN}^{-}$binding at alkaline $\mathrm{pH}$ is more difficult to interpret since several changes are occurring. First, $\mathrm{HCN}$ ionizes to $\mathrm{CN}^{-}$possibly making it more difficult to diffuse into the active site and/or bind to the heme iron. Second, WT CPO undergoes an alkaline transition that seems to differ from that in the mutants judging from the spectra at $\mathrm{pH}$ 12.0. My results for $\mathrm{CN}^{-}$binding at alkaline $\mathrm{pH}$ are consistent with decreased $\mathrm{CN}^{-}$affinity to the WT (Kd of $4 \mathrm{mM}$ ) reported at $\mathrm{pH} 9.5$ (174). However, it should be noted that the cyanide derivative of the WT CPO at $\mathrm{pH} 9.5$ is different from that at $\mathrm{pH} 12.0$ since 
its Soret peak is $15 \mathrm{~nm}$ red-shifted from that in the WT-CN complex at pH 12.0 (437 nm vs. $422 \mathrm{~nm}$ ), and the second alkaline transition in WT CPO takes place at $\mathrm{pH} 11$, thus $\mathrm{CN}^{-}$ binds to two different forms of CPO.

Table 3.4. UV-Vis spectral properties of ferric WT and mutant CPO and their cyanide complexes at $\mathrm{pH} 6.0$ and $\mathrm{pH}$ 12.0. (s), (n.s), and (s.s) denote the presence of a shoulder, no shoulder, or a slight shoulder, respectively.

\begin{tabular}{|c|c|c|c|c|c|}
\hline Protein & $\begin{array}{c}\delta \text { band } \\
(\mathrm{nm})\end{array}$ & $\begin{array}{l}\text { Soret } \\
(\mathrm{nm})\end{array}$ & $\begin{array}{c}\beta \text { band } \\
(\mathrm{nm})\end{array}$ & $\begin{array}{c}\alpha \text { band } \\
(\mathrm{nm})\end{array}$ & $\begin{array}{l}\text { CT } \\
(\mathbf{n m})\end{array}$ \\
\hline WT CPO (pH 6) & -- & 398 & 515 & 550 & 650 \\
\hline WT-CN (pH 6) & 364 & 439 & -- & 564 & -- \\
\hline WT CPO (pH 12) & 361 & 422 & -- & 547 & -- \\
\hline WT-CN (pH 12) & 361 & 422 & -- & 547 & -- \\
\hline R26A (pH 6) & 354 & $\begin{array}{c}418 \\
\text { (s) }\end{array}$ & 540 & 568 & 644 \\
\hline R26A-CN (pH 6) & 364 & $436 \rightarrow 432$ & -- & 560 & -- \\
\hline N33A (pH 6) & 356 & $\begin{array}{l}420 \\
\text { (n.s) } \\
\end{array}$ & 540 & 575 & -- \\
\hline N33A-CN (pH 6) & 364 & $437 \rightarrow 427$ & -- & 557 & -- \\
\hline N33A (pH 12) & $\begin{array}{l}363 \text { (as a } \\
\text { shoulder) }\end{array}$ & 385 & -- & -- & -- \\
\hline N33A-CN (pH 12) & 363 & 422 & -- & 547 & -- \\
\hline R26A/N33A (pH 6) & 356 & $\begin{array}{l}421 \\
(\mathrm{~s} . \mathrm{s})\end{array}$ & 540 & 575 & $\begin{array}{l}644 \\
\text { (low) }\end{array}$ \\
\hline R26A/N33A-CN (pH 6) & 364 & $439 \rightarrow 437$ & -- & 555 & -- \\
\hline R26A/N33A (pH 12) & 360 & 422 & 543 & 578 & 646 \\
\hline $\begin{array}{l}\mathrm{R} 26 \mathrm{~A} / \mathrm{N} 33 \mathrm{~A}-\mathrm{CN} \quad(\mathrm{pH} \\
12)\end{array}$ & 360 & $436 \rightarrow 430$ & -- & 555 & -- \\
\hline
\end{tabular}

Interestingly, the spectrum of the WT after transition at $\mathrm{pH} 7.5$ to the low-pin form is similar to the spectra of the mutants at $\mathrm{pH} 6.0$ that also possess a low-spin heme iron, and the cyanide Kd values for these CPO forms are comparable (4 mM for the WT vs. $3.6 \mathrm{mM}$, 
$3.8 \mathrm{mM}$, and $10.5 \mathrm{mM}$ for the mutants). However, the WT and mutant low-spin forms must be different since the low-spin WT is enzymatically inactive, while the mutants are active.

\subsection{Conclusion}

H-bonding networks within the active sites are conserved features in many heme

proteins. Such networks in the distal and proximal heme sides of peroxidases and cytochrome P450s are important for their structure and catalysis. My study examined the contributions of the H-bonding network formed by Arg 26-Asn 37 and Ala 27-Asn33 on the proximal side of CPO to the structural and thus spectroscopic properties of the enzyme. The R26A, N33A, and R26A/N33A CPO were characterized with various spectroscopic techniques and compared to the WT.

My results have shown that removal of H-bonds in either of the residue pairs does not significantly affect secondary structure. However, alpha-helical content is dramatically increased if all H-bonds are removed. Additionally, all mutants exhibit differences in tertiary CD, which indicates that the proximal H-bond network is important for maintaining the tertiary structure of $\mathrm{CPO}$, which in turn influences the environment of the active site. Carbon monoxide and cyanide binding results have demonstrated that removal of H-bonds in the proximal site of CPO induces structural changes that have a larger effect on binding of anionic ligand than on binding of a neutral ligand.

It is likely that removal of the proximal H-bonds changes the flexibility of helix A, affecting its position relative to the heme and influencing its interactions with the protein matrix. The dislocation of the helix A is likely to change the bond length between the iron and the proximal thiolate, which in turn would affect the heme environment and thus the catalytic properties of CPO discussed in the next chapter. 


\section{CHAPTER IV.}

\section{EFFECTS OF HYDROGEN BOND NETWORK IN THE PROXIMAL HELIX ON CATALYTIC PROPERTIES OF CHLOROPEROXIDASE}

\subsection{Background}

As mentioned in Chapter I, CPO is a heme-thiolate protein that belongs to the peroxidase-peroxygenase family of enzymes $(28,71)$. Many heme enzymes are capable of efficiently catalyzing one or two particular types of reactions, depending on the class of the enzyme. However, CPO is capable of catalyzing a plethora of reactions such as halogenation, peroxidation, peroxide dismutation, and epoxidation that are characteristic of multiple enzyme classes $(112,113,141,142)$. The aforementioned reactions and other oxidations are catalyzed by the reactive form of CPO, Cpd I, which is formed by binding of hydrogen peroxide to the ferric resting state of the protein. The proximal oxygen of the peroxide is deprotonated by Glu 183, producing the so called Compound 0 (Cpd 0), followed by an initial homolytic O-O bond scission. The homolytic process becomes heterolytic as the proton which was removed from the proximal oxygen, is transferred to the distal oxygen, and a water moiety departs, producing the oxo-ferryl cationic-radical intermediate, Cpd I (106, 226).

The formation rates and reactivity of Cpd I depend on the pKa of the distal ligand and electron-donating ability of the proximal ligand $(204,244)$. The higher basicity of the distal ligand $\left(\mathrm{H}_{2} \mathrm{O}_{2}\right)$ would result in slower formation of Cpd 0 since proton removal from peroxide-bound ferric heme would be more difficult. However, if the pKa is too low it would result in slower formation of Cpd I from Cpd 0 because of the difficulty in protonating Cpd 0. Additionally, the electronegativity of the proximal thiolate creates a so- 
called 'push effect' that directly influences distal ligand pKa and Cpd I redox potential (234, 244-246). The 'push' provided by the proximal thiolate increases the basicity of the distal oxygen in Cpd 0, thus facilitating water release and Cpd I formation. However, the 'push' concurrently decreases the redox potential and thus the reactivity of Cpd I. To modulate the 'push' for the optimal activity without preventing formation of Cpd 0, CPO and other heme thiolate enzymes evolved mechanisms for reducing thiolate electronegativity via Cys-amide H-bonds and the electropositive environment of the Nterminus of the proximal helix (244).

Hydrogen bonding between a proximal Cys and polypeptide amides is a common feature of many heme thiolate proteins including CPO, P450s, and NO synthase (71, 98, 247). In CPO, Cys 29 is H-bonded to the peptide NH groups of Ala 31 and Leu 32 situated at the N-terminus of helix A, while in P450cam the proximal Cys 357 is H-bonded to $\mathrm{NH}$ groups of Leu 358, Gly 359, and Gln 360 (71, 98). These H-bonds contribute to the increase of the iron redox potential by decreasing the negative charge on the Cys ligand, and stabilize the thiolate by preventing its protonation and protecting against diatomic reactants, all of which result in the formation of inactive forms of the enzyme $(101,102)$. As demonstrated by mutation studies on P450cam by Yoshioka et al., removal of thiolateamide bonds in L358P, Q360P, and Q360L mutants results in increased push effect and reduced redox potential of the enzyme $(101,211)$.

Once Cpd I is formed in CPO, it is able to follow different catalytic pathways. For example, Cpd I can either form oxo-ferryl (possibly, hydroxy-ferryl) Cpd II in the peroxidation pathway, oxo-ferric Cpd $\mathrm{X}$ in halogenation pathway, or return to its resting state via dismutation and P450 pathways $(104,107,109)$. One-electron oxidations that 
occur via the peroxidation pathway include peroxidation and oxidative de-halogenation reactions and have been proposed to occur on the surface of the enzyme (112, 127, 137, 138). Although Cpd II, an intermediate formed during one-electron oxidations, is postulated to be an oxo-ferryl species, its protonation state is still debated. Computational studies $(107,108)$ have suggested the iron(IV)-hydroxide species to be the major form of Cpd II; however, this hypothesis has not been confirmed experimentally. Two-electron oxidations include a variety of reactions such as halogenation, epoxidation, sulfoxidation, peroxide dismutation, and are suggested to occur at the active site of CPO, except for halogenations (112, 113, 141, 142). Compound $\mathrm{X}$, ferric $\mathrm{Fe}-\mathrm{HOCl}$, is formed during chlorination reactions catalyzed by CPO (109). No intermediates besides Cpd I have been identified in other two-electron oxidations. The ability of CPO to catalyze such diverse reactions is postulated to be a result of its unique active site structure.

Chloroperoxidase is folded into $\mathrm{N}$ - and C-terminal domains with the heme group located between the domains. The active site of CPO is a peroxidase-P450 hybrid with a peroxidase-like polar distal environment and a P450-like proximal thiolate ligation (71). The proximal heme ligand, Cys 29, is located in the N-terminus of helix A, which is almost perpendicular to the heme plane (71). Interestingly, in cytochromes P450 and most other peroxidases with the imidazolate ligation, the helix containing the proximal ligand is situated in the C-terminal domain and is parallel to heme (98). The electropositive helix environment reduces the electronegativity of the proximal Cys, thus contributing to the increase in redox potential, and has been termed the 'proximal helix effect'. The almost perpendicular orientation of the proximal helix to the heme in CPO provides a larger increase in redox potential than observed in P450 with its parallel helix $(99,100,248)$. 
Thus, Cys-amide H-bonds together with the proximal helix dipole have a significant influence on the reactivity of CPO.

Hydrogen bond networks are important structural features in heme imidazolate enzymes as well. In many peroxidases, the distal His-Arg pair is essential for efficient production of Cpd I (64-66). In CPO, the role of the His-Arg pair is carried out by Glu 183 H-bonded to His 105. Both Arg in peroxidases and His in CPO provide a particular orientation to the catalytic His and Glu, respectively, and aid in achieving optimal rates of Cpd I formation (202, 249, 250). Proximal H-bond networks consisting of His-AspTrp/Phe and His-Asn-Arg are conserved in peroxidases-catalases and peroxidasescyclooxygenases, respectively. These networks are believed to stabilize higher oxidation states of Cpd I and Cpd II by de-protonating the proximal His at $\mathrm{N} \delta$ position and maintaining shorter Fe-imidazolate bond distances in peroxidases as compared to globins that lack such a network $(251,252)$.

There are several amino acids in the proximal helix of CPO that also form a H-bonding network, in particular Arg 26-Asn 37 and Ala 27-Asn 33. It is reasonable to suggest that this H-bond network provides structural support for the proximal helix and helps its positioning in a particular orientation relative to the heme, possibly influencing the catalytic properties of the enzyme. However, no research has been done on the role of this proximal H-bond network in defining the biochemical properties of CPO.

In this study, I investigated how deletion of H-bonds between Arg 26-Asn 37 and Ala 27-Asn 33 affect catalytic properties of CPO. I replaced Arg 26 and Asn 33 with Ala to disrupt H-bonding and characterized R26A, N33A, and R26A/N33A CPO using various 
enzymatic assays and UV-Vis spectrophotometry. My results show that the H-bonding network of the proximal helix is important for maintaining the catalytic diversity of CPO.

\subsection{Experimental procedures}

All the chemicals and materials used are described in section 2.2.1.

\subsubsection{Construction of R26A, N33A, and R26A/N33A CPO mutants}

To investigate the role of the hydrogen bond network in the proximal helix of CPO (Arg 26-Asn 37 and Ala 27-Asn 33) in catalysis, R26A, N33A, R26A/N33A mutants were constructed. Gene construction, expression, and purification of the mutants were performed as described in sections 3.2.1 and 3.2.2.

\subsubsection{Enzymatic assays}

To investigate catalytic properties of the mutants lacking hydrogen bonds in the proximal helix, enzymatic assays described in section 2.2.7 were performed with the following modifications. Enzyme amounts used for the ABTS assay at various $\mathrm{pH}$ are listed in Table 4.1.

Table 4.1. Enzyme amounts used in ABTS assay at different $\mathrm{pH}$.

\begin{tabular}{lll}
\hline $\mathbf{p H}$ & $\mathbf{W T}(\boldsymbol{\mu M})$ & Mutant $(\boldsymbol{\mu M})$ \\
\hline $2.75,3.0$ & 0.03 & 0.03 \\
\hline 3.5 & 0.1 & 0.03 \\
\hline $4.0,4.5$ & 0.5 & 0.03 \\
\hline $5.0,5.5$ & 2.0 & 0.1 \\
\hline 6.0 & 5.0 & 0.2 \\
\hline
\end{tabular}

Styrene epoxidation assay (15) was performed in $100 \mathrm{mM}$ phosphate-citrate or phosphate buffer (pH 2.75, 3.0, 3.5, 4.0, 4.5, 5.0, 5.5, 6.0, 7.0, 8.0). Protein concentration 
was $0.3 \mu \mathrm{M}$. MCD assay was performed in $100 \mathrm{mM}$ phosphate-citrate or phosphate buffer (pH 2.75, 3.0, 3.5, 4.0, 4.5, 5.0). Protein concentration was $0.6 \mu \mathrm{M}$. Finally, dismutation assay was performed in $100 \mathrm{mM}$ phosphate-citrate or phosphate buffer $(\mathrm{pH}$ 2.4, 3.0, 3.5, 4.0, 4.5, 5.0, 5.5, 6.0). Protein concentration was $0.5 \mu \mathrm{M}$.

\subsection{Results and discussion}

In general, catalytic properties of heme proteins are influenced by the heme iron redox potential, architecture of the active site, and electrostatic interactions present in the distal and proximal sides $(48,106,207,244)$. To examine the effect of the proximal hydrogen bonding on catalytic properties of CPO, chlorination (MCD), dismutation $\left(\mathrm{H}_{2} \mathrm{O}_{2}\right)$, peroxidation (ABTS), and epoxidation (styrene) assays were performed.

\subsubsection{Chlorination and dismutation activities of $\mathrm{WT}$ and mutant $\mathrm{CPO}$ at various $\mathrm{pH}$}

The chlorination reaction catalyzed by WT CPO is most efficient at very acidic $\mathrm{pH}$ with highest rates at $\mathrm{pH}$ 2.75. Chlorination proceeds via one two-electron step with the formation of Cpd I and Cpd X $(109,141)$. Thus, the rate of chloride oxidation depends on the formation rates of the two intermediates. Since the rate of Cpd I formation has been shown to be independent of $\mathrm{pH}(104,106)$, Cpd X must be responsible for differences in activities observed at various $\mathrm{pH}$. Indeed, it has been demonstrated that only protonated Cpd X is able to chlorinate MCD (109), which explains WT's high chlorination activity at acidic pH. In contrast, the mutant CPOs (R26A, N33A, and R26A/N33A) lacking H-bonds in the heme proximal helix almost completely abolish chlorination activity at all $\mathrm{pH}$ values checked (2.4-5.0, Figs. 4.1-4.2). The loss of chlorination activity may be explained by the decrease in the Cpd I redox potential that is affected by the 'push', spin state, and the extent of heme burial. 


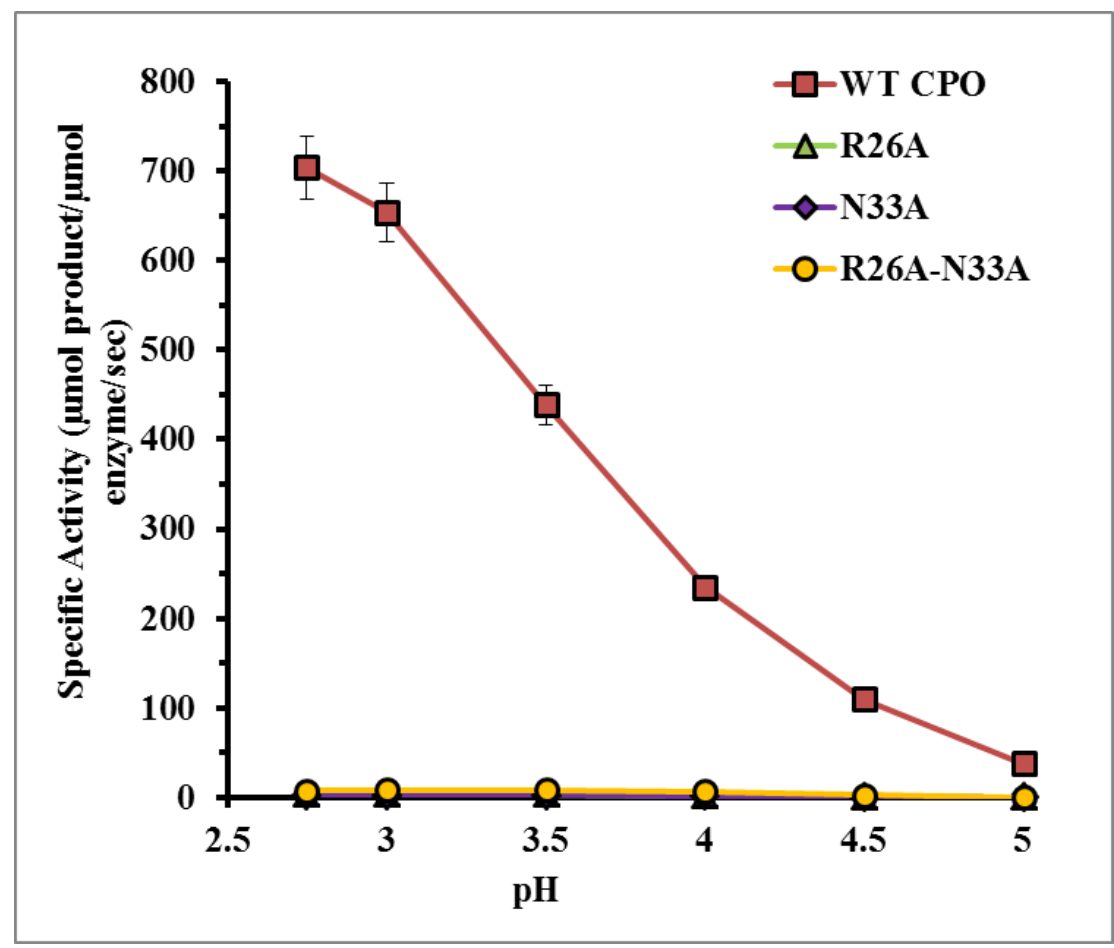

Figure 4.1. pH profiles of WT and mutant CPO chlorination activities (some error bars are smaller than the point size and are not visible on the graph).

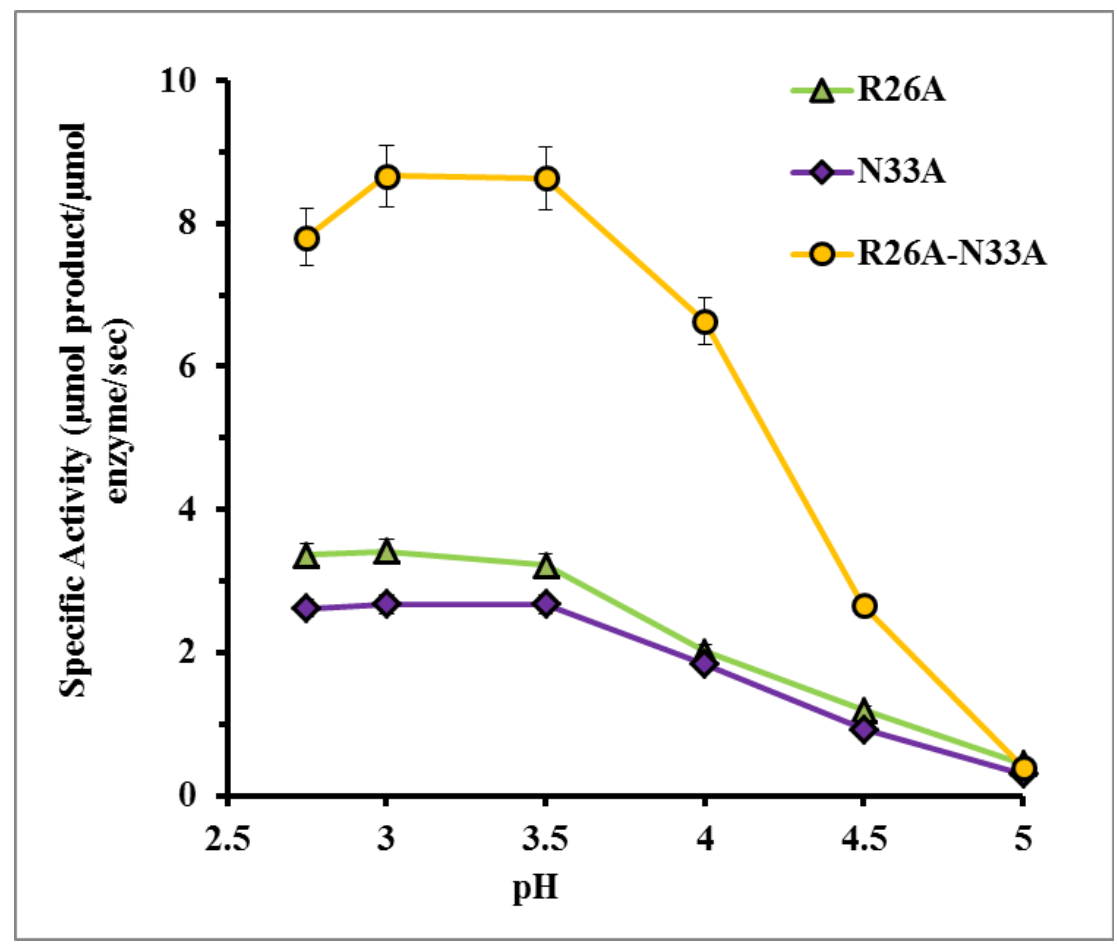

Figure 4.2. $\mathrm{pH}$ profiles of R26A, N33A, and R26A/N33A CPO chlorination activities (some error bars are smaller than the point size and are not visible on the graph). 


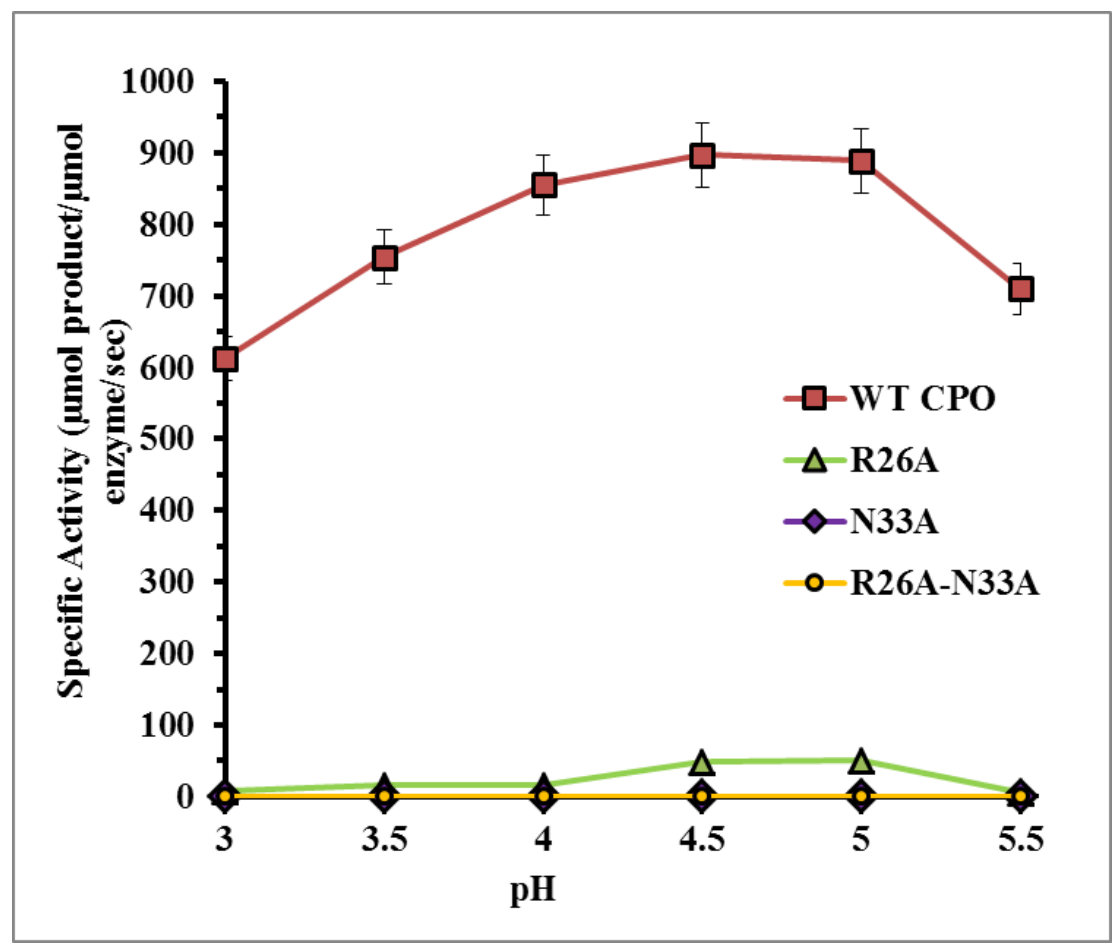

Figure 4.3. $\mathrm{pH}$ profiles of WT, R26A, N33A, and R26A/N33A CPO dismutation activities (some error bars are smaller than the point size and are not visible on the graph).

Since disruption of the H-bond network in the heme proximal helix affects tertiary structures of the mutants, it is likely that perturbations in the active site cause increase in the electron push from the thiolate ligand, which in turn decreases redox potential. For example, removal of the H-bonds may affect the positioning of the proximal helix, whose dipole contributes to the thiolate 'push’ decrease. Changes in helix A orientation may result in a smaller electron density withdrawal from the heme that would reduce the redox potential.

In P450s with a parallel position of the proximal helix relative to the heme, the helix dipole results in only a $70 \mathrm{mV}$ redox potential increase of $\mathrm{Fe}(\mathrm{III}) / \mathrm{Fe}(\mathrm{II})$ couple as opposed to $130 \mathrm{mV}$ in CPO whose proximal helix is perpendicular to the heme (204). In addition, since the proximal helix of CPO contains Cys 29 ligated to the heme, changes in the 
position of the helix may affect the orientation of the proximal thiolate, which in turn can affect the heme environment.

Tertiary structural changes, observed by CD, could have affected the heme exposure to the solvent, which would also contribute to the decrease of both the redox potential and catalatic activity of the enzyme $(253,254)$. This is consistent with negligible dismutation activity of the mutants (Fig. 4.3).

Hydrogen peroxide decomposition, a two-electron oxidation reaction, is catalyzed by WT CPO most efficiently at acidic $\mathrm{pH}$ with the highest rates at $\mathrm{pH}$ 4.5-5.0. N33A and R26A/N33A do not have any dismutation activity in the acidic $\mathrm{pH}$ range, while R26A mutant has a very low activity at $\mathrm{pH}$ 4.5-5.0. The low catalatic activity of R26A indicates that it might have a slightly higher redox potential than the other two mutants, which would allow peroxide dismutation though not be high enough for chloride oxidation.

\subsubsection{Peroxidation and epoxidation activities of WT and mutant $\mathrm{CPO}$ at various pH}

The optimal $\mathrm{pH}$ range for ABTS peroxidation by the WT CPO is 2.75-3.0. The activity of WT CPO is decreased more than 10 -folds at $\mathrm{pH} 3.5$, and peroxidation is negligible at $\mathrm{pH}$ above 4.0. In contrast, R26A and N33A possess significantly higher peroxidation ability in $\mathrm{pH}$ range 3.5-5.0 with the optimal activity at $\mathrm{pH} 3.5$ while $\mathrm{R} 26 \mathrm{~A} / \mathrm{N} 33 \mathrm{~A}$ is more active at all pH checked (Fig. 4.4). The peroxidation activity of the double mutant is about 2-fold higher than that of R26A and N33A at pH 3.5.

The shift in optimal $\mathrm{pH}$ for peroxidation activity can be explained by the possible increase in the pKa of Cpd II. High activity of the WT below pH 3.5 suggests that protonation of Cpd II might be required for the peroxidation reaction to occur, similarly to the protonation of Cpd X in the halogenation pathway, and that Cpd II has a low pKa 
(below 3.5). In mutant CPOs, the pKa of Cpd II might be higher, and that would result in the formation of the active protonated Cpd II at higher $\mathrm{pH}$. Hence, peroxidation is able to occur over a wider $\mathrm{pH}$ range. This observation is also consistent with the proposed inverse relation of the heme redox potential and pKa of the distal ligand. The lower redox potential of mutant CPOs that can be inferred from their inability to oxidize chloride would increase the pKa of the distal ligand.

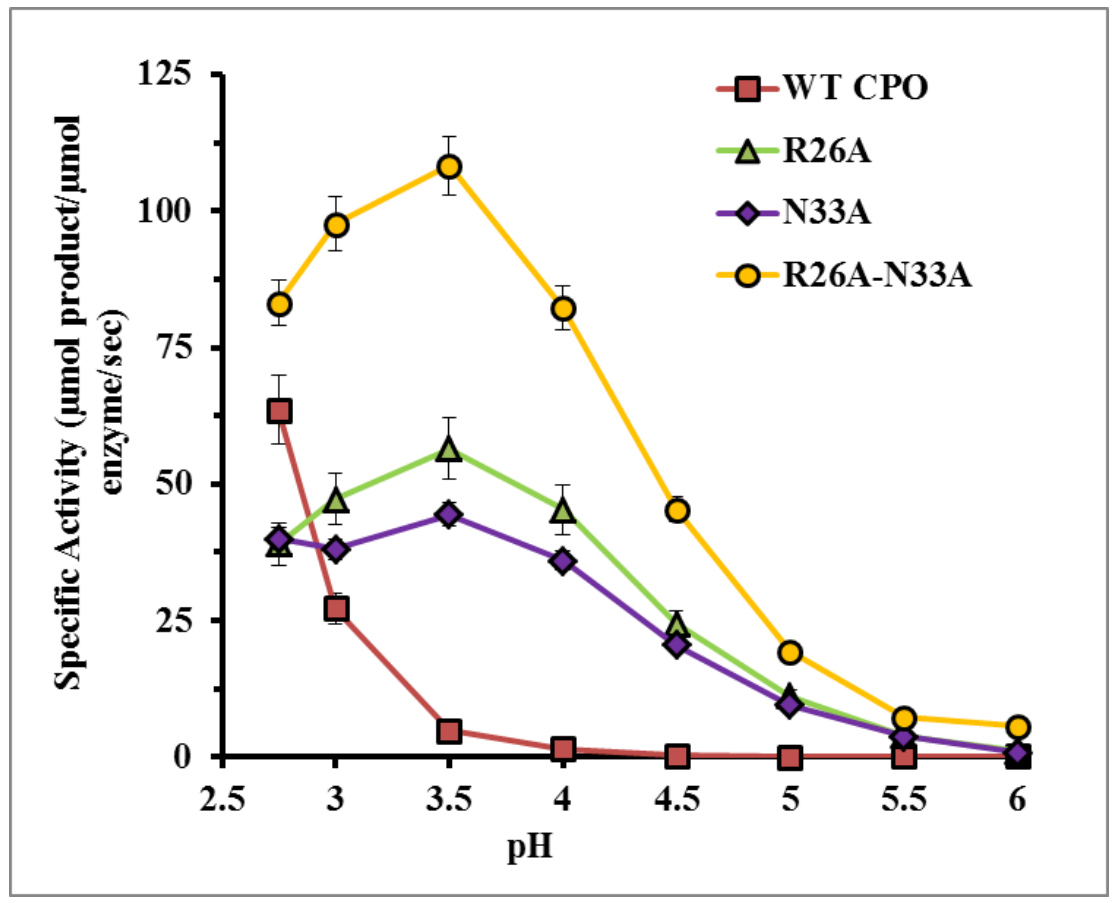

Figure 4.4. $\mathrm{pH}$ profiles of $\mathrm{WT}$ and mutant $\mathrm{CPO}$ peroxidation activities (some error bars are smaller than the point size and are not visible on the graph).

Epoxidation of styrene, catalyzed by mutant CPOs, occurs at significantly higher rates as compared to the WT. Wild-type CPO oxidizes styrene over a wide $\mathrm{pH}$ range, 2.75-7.0 (Fig. 4.5). A significant decrease in activity is observed at $\mathrm{pH}$ higher than 7.0 because of the formation of the inactive alkaline species of CPO. The rates of styrene epoxidation do not seem to heavily depend on $\mathrm{pH}$ since the difference between specific activities, for example, at pH 6.0 and 2.4 is only 2-fold. A similar situation is observed in epoxidations 
catalyzed by the mutants, which are active in the $\mathrm{pH}$ range 2.4-9.0 with specific activities varying only about 1.5-fold (Fig. 4.5). This observation indicates that epoxidation does not require a catalytic intermediate, whose protonation state determines the reaction rates, as has been observed in chlorination and peroxidation assays. Also, it should be noted that in the case of mutant CPOs, the enzymes remain very active at $\mathrm{pH} 9.0$, suggesting that mutant CPOs do not form the inactive alkaline form similarly to WT CPO. This result is also confirmed by electronic absorption spectra of the mutants showing that native enzyme forms start changing only at $\mathrm{pH} 10.0$.

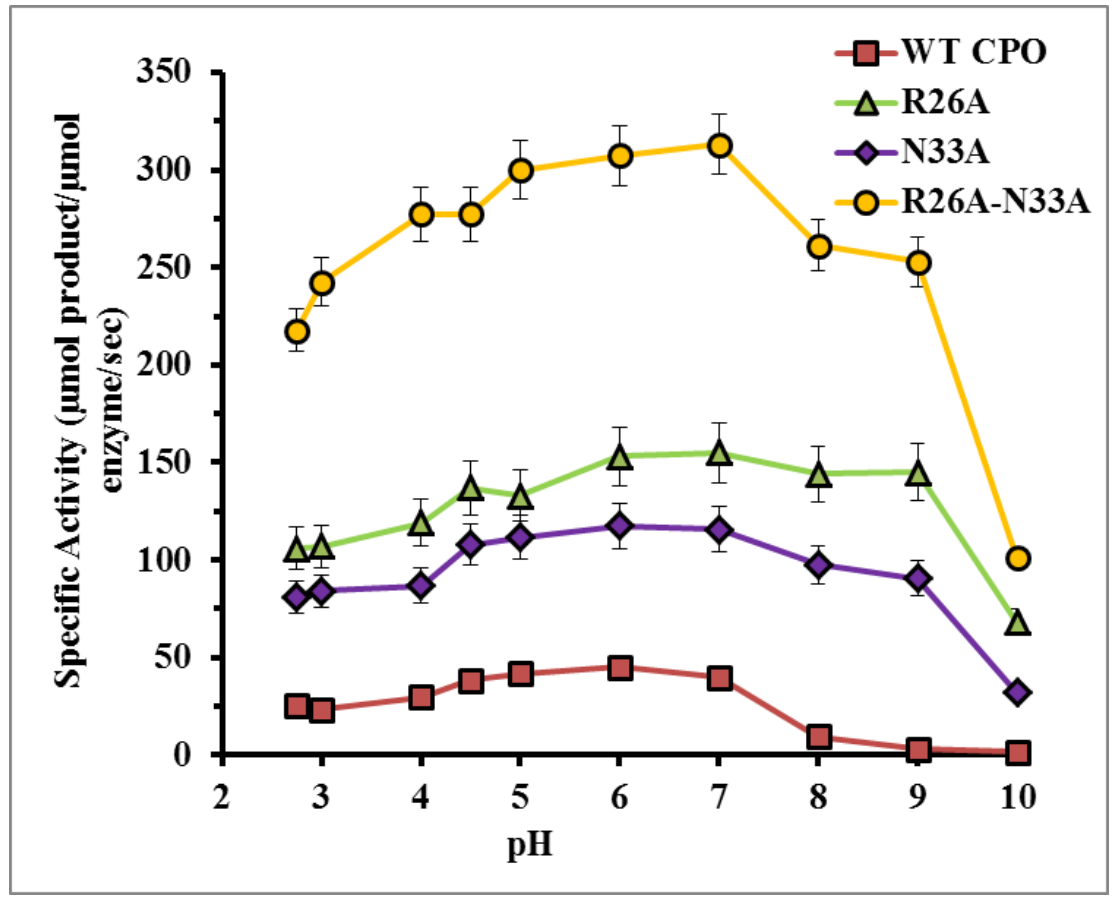

Figure 4.5. pH profiles of WT and R26A CPO epoxidation activity (some error bars are smaller than the point size and are not visible on the graph).

In mutant CPOs, the lack of catalatic activity, which competes with other reactions for Cpd I, is expected to contribute to the increase in peroxidation and epoxidation rates. During hydrogen peroxide decomposition, a molecule of hydrogen peroxide binds the ferric enzyme, and Cpd I is formed. Next, another molecule of hydrogen peroxide binds to 
Cpd I. Hydrogen peroxide is decomposed into water and oxygen while Cpd I is converted back to the ferric resting state. This process results in premature depletion of the oxidizing agent necessary for the formation of Cpd I, which is required for peroxidation and epoxidation. When dismutation is absent, more Cpd I is available in the reaction and hydrogen peroxide is not depleted via the dismutation pathway.

As judged from tertiary CD (chapter 3), another possible contribution to the increase in epoxidation activity of the mutants is the changes in accessibility of the active site by substrates, which is important for two-electron oxidations by heme enzymes (237). Large changes in the secondary structure of the double mutant, having the highest epoxidation rates, are consistent with this hypothesis.

\subsubsection{Comparison of WT and mutant CPO activities at optimal pH}

pH 2.75 and $\mathrm{pH} 4.5$ were chosen to compare specific activities of WT and the mutants since these are the optimal $\mathrm{pH}$ values for chlorination/peroxidation and epoxidation/dismutation activities, respectively. Furthermore, the majority of specific activities of the WT and other mutant CPOs reported in the literature were measured at $\mathrm{pH}$ 2.75 (chlorination) and $\mathrm{pH} 4.5$ (dismutation, peroxidation, epoxidation). Hence, my results for mutant activities can be compared to the activities of other CPO mutants.

At $\mathrm{pH} 2.75$, R26A and N33A possess about $60 \%$ peroxidation activity of that of the WT (Fig. 4.6). However, at pH 4.5, R26A and N33A showed around 80-fold and 70-fold increase in peroxidation activity, respectively (Fig. 4.7). The double mutant, R26A/N33A, is more active than the WT at both $\mathrm{pH} 2.75$ and 4.5, showing 1.3-fold and an astounding 250-fold increase in peroxidation, respectively (Figs. 4.6-4.7). All mutants are more active in epoxidation assay as well, showing 3.5-fold, 2.8-fold, and 7.2-fold increase (R26A, 
N33A, and R26A/N33A, respectively) (Fig. 4.8). Different trends are observed in chlorination and dismutation assays.

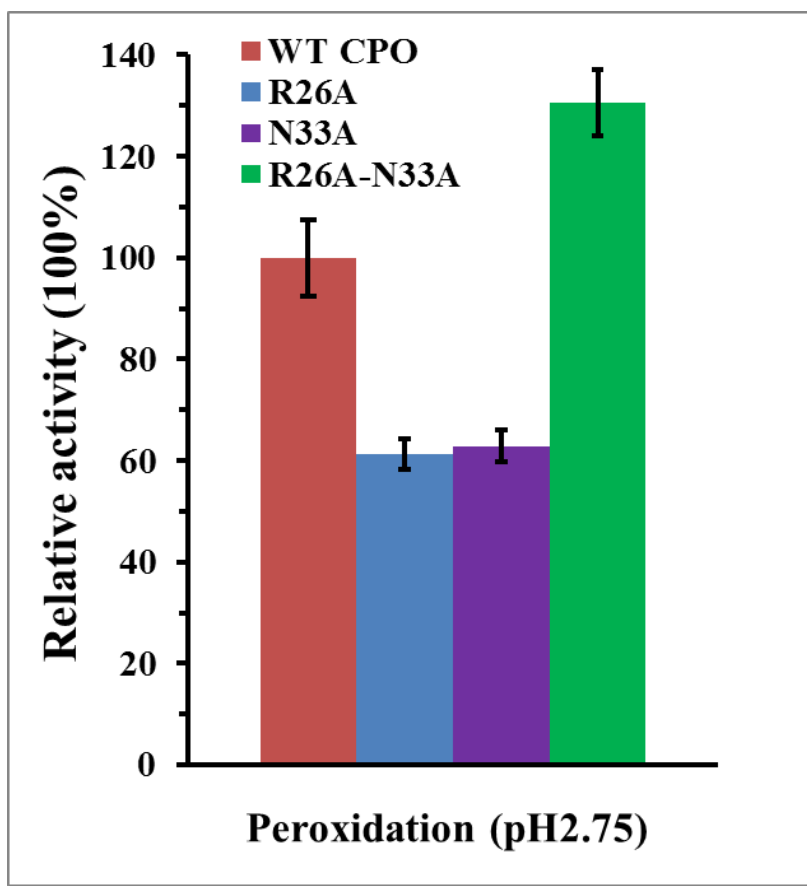

Figure 4.6. Relative peroxidation activities of the WT and mutant CPOs at pH 2.75.

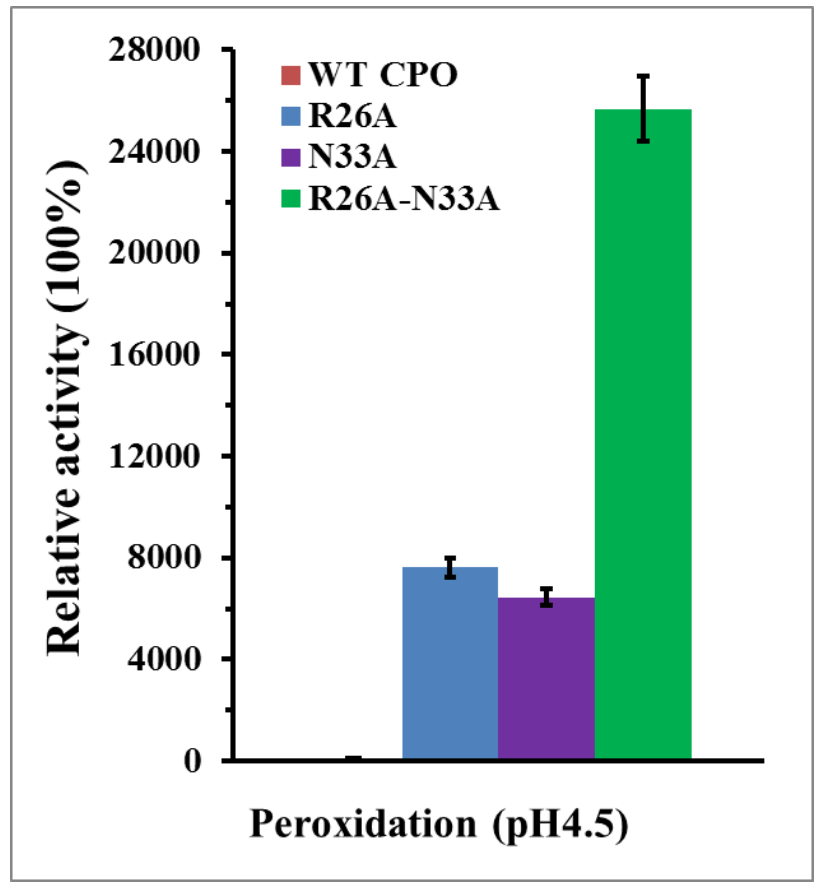

Figure 4.7. Relative peroxidation activities of the WT and mutant CPOs at pH 4.5. 


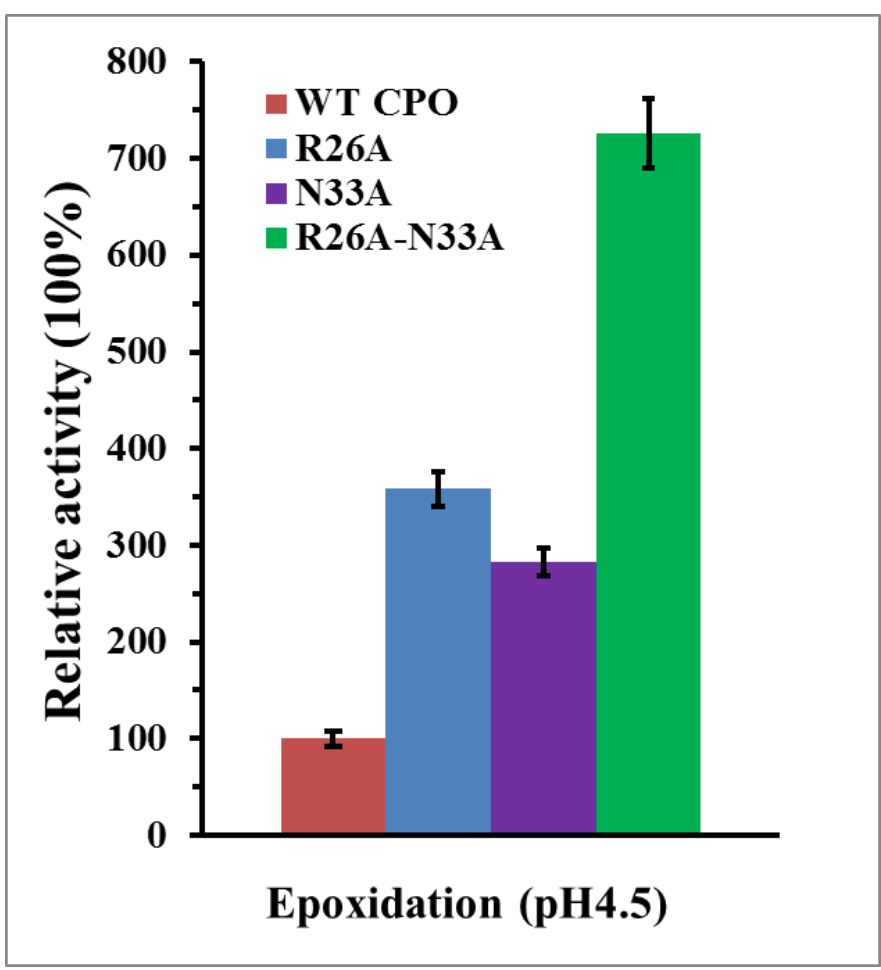

Figure 4.8. Relative epoxidation activities of the WT and mutant CPOs at $\mathrm{pH} 4.5$.

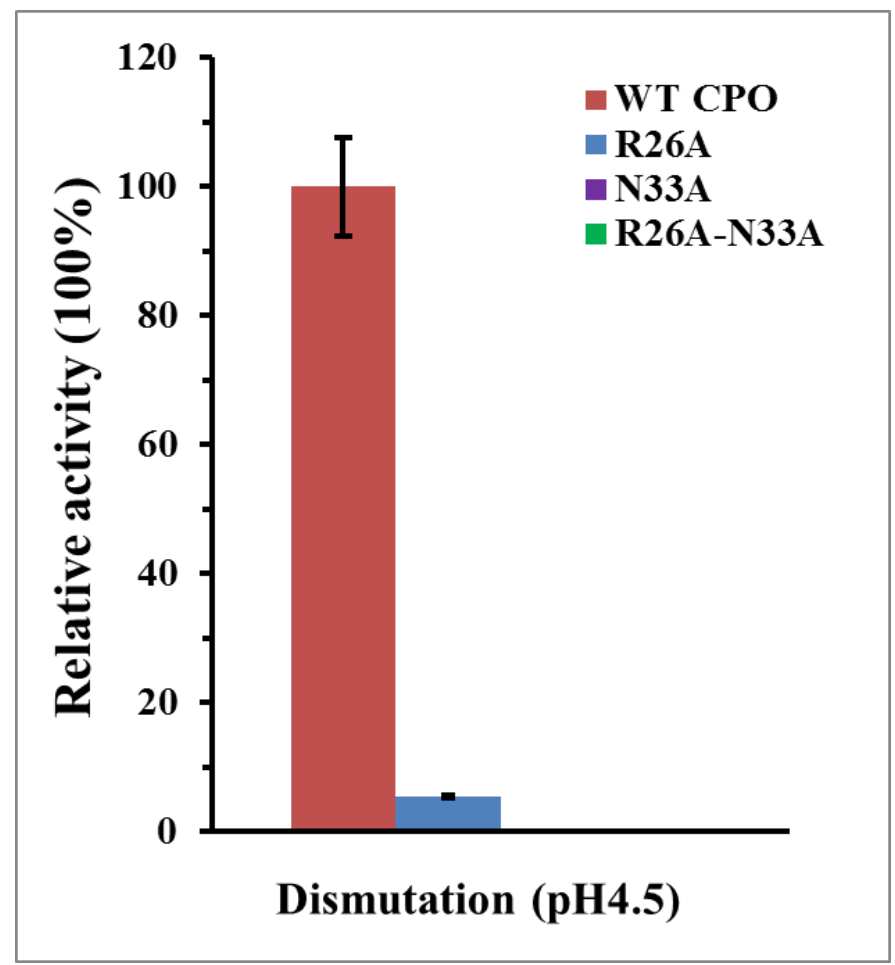

Figure 4.9. Relative dismutation activities of the WT and mutant CPOs at $\mathrm{pH} 4.5$. 


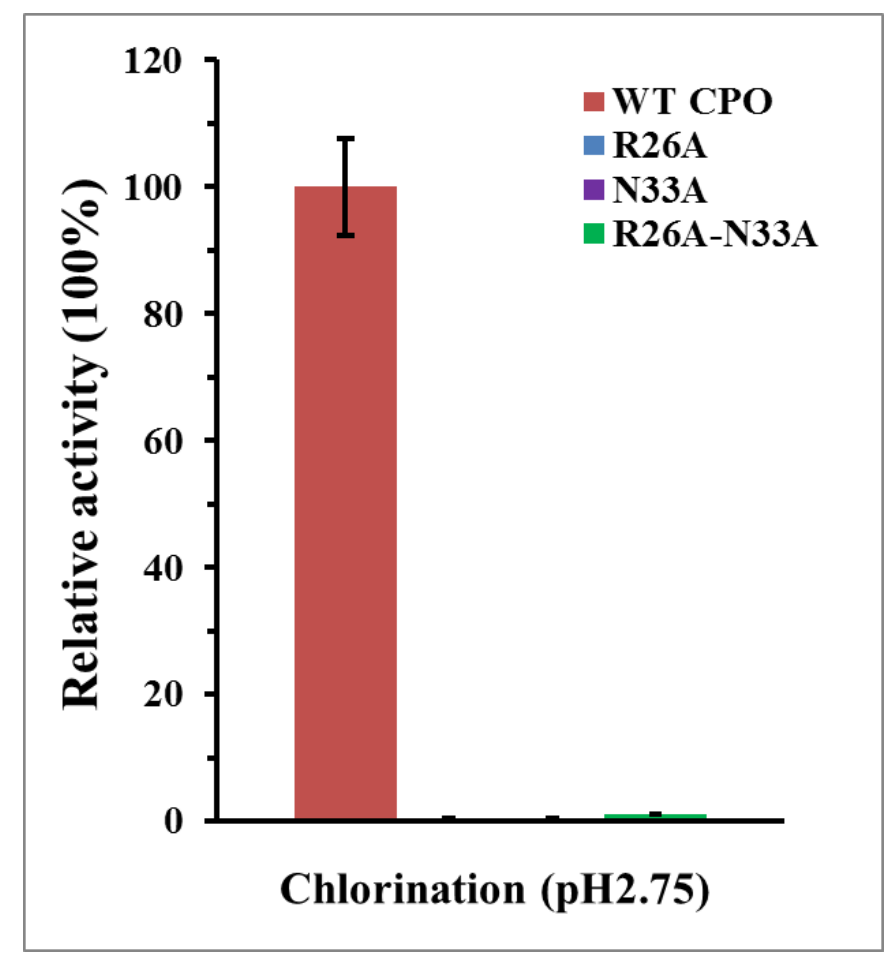

Figure 4.10. Relative chlorination activities of the WT and mutant CPOs at pH 2.75.

R26A shows a slight dismutation activity (5\% of that of the WT) (Fig. 4.9). All mutants possess negligible chlorination activity (Fig. 4.10). These results suggest that the redox potentials of R26A, N33A, and R26A/N33A mutants might be lower than that of the WT.

Overall, my results indicate that H-bond network in the proximal helix A of CPO plays an important role in maintaining the secondary and tertiary structures of CPO and contributes to its high chlorination and dismutation activities. However, the presence of these H-bonds also results in decreased epoxidation and peroxidation rates, especially at slightly acidic and neutral $\mathrm{pH}$.

\subsection{Conclusion}

There has been quite a substantial amount of work published on heme protein catalysis over the years. It is clear that heme proteins evolved multiple mechanisms that work 
together to carry out their particular biological functions. Many factors that influence catalytic properties of heme enzymes have been identified including types of electronic structures, nature of heme ligands, electrostatic interactions, and H-bond networks. However, the importance and particular contributions of many structural features in catalysis remain to be delineated. No research has been published on the role of the H-bond network in the proximal helix of CPO on its catalysis. In this work, I employed rational design to investigate the role of this H-bond network in the catalytic properties of CPO. Three CPO mutants (R26A, N33A, and R26A/N33A) were engineered and characterized using various enzymatic assays. My results have shown that the proximal H-bond network is as important for the catalytic diversity of $\mathrm{CPO}$ as the proximal thiolate ligand, and is also responsible for providing $\mathrm{CPO}$ with its high chlorination and dismutation activities. My research contributes to the ongoing exploration of factors that influence heme catalysis and helps us better understand the structure-function relations in heme-thiolate proteins in general and in CPO in particular. 


\section{CHAPTER V.}

\section{PERSPECTIVES}

The results of this work show that several structural features of the proximal heme pocket, particularly the axial thiolate and H-bond network, strongly influence the ligandbinding and catalytic properties of CPO. Although C29, R26, and N33 in the proximal site do not directly participate in catalysis, they influence the structure of the protein and the heme environment, which in turn significantly affect the catalytic behavior of CPO.

The CPO mutants investigated in this work (C29H, R26A, N33A, and R26A/N33A) can be further studied by RR and NMR spectroscopies to obtain more information about the structure of the active site. Solving crystal structures of the mutants would help better understand ligand-binding and catalysis results. The ligand-binding properties of mutant CPO can be further investigated with thiocyanate, nitric oxide, halides, etc. to gain insights into factors affecting ligand binding. Catalytic behavior can be further explored with other reactions such as sulfoxidation, hydroxylation, bromination together with various substrates. The enantioselectivity of oxygen insertions would present an attractive target for the pharmaceutical and chemical industries. Investigations of CPO with other point mutations in the active site or disulfide bond region would be also interesting.

Mutant CPO production requires large amounts of media because of much lower protein yields as compared to the WT. Hence, determination of factors influencing the expression of CPO mutants and their secretion in the holo form, and optimization of fungal expression systems would help increase mutant CPO yields, encouraging further research on CPO and other heme proteins, especially those ones that are understudied because of the difficulties associated with obtaining good yields. 


\section{REFERENCES}

1. Antonini, E. and Brunori, M. Hemoglobin and myoglobin in their reactions with ligands. North-Holland Publishing Co.: Amsterdam, 1971.

2. Perutz, M. Myoglobin and haemoglobin: role of distal residues in reactions with haem ligands. Trends Biochem. Sci. 1989, 14, 42-44.

3. Wittenberg, J. and Wittenberg, B. Mechanisms of cytoplasmic hemoglobin and myoglobin function. Annu. Rev. Biophys. Biophys. Chem. 1990, 19, 217-241.

4. Appleby, C.A. Leghemoglobin and Rhizobium respiration. Annu. Rev. Plant Physiol. 1984, 35, 443-478.

5. Burmester, T., Weich, B., Reinhardt, S., Hankeln, T. A vertebrate globin expressed in the brain. Nature 2000, 407, 520-523.

6. Kawada, N., Kristensen, D. B., Asahina, K., Nakatani, K., Minamiyama, Y., Seki, S., Yoshizato, K. Characterization of a stellate cell activation-associated protein (STAP) with peroxidase activity found in rat hepatic stellate cells. $J$. Biol. Chem. 2001, 276, 25318-25323.

7. Burmester, T., Ebner, B., Weich, B., Hankeln, T. Cytoglobin: a novel globin type ubiquitously expressed in vertebrate tissues. Mol. Biol. Evol. 2002, 19, 416-421.

8. Keilin, D. Cytochrome and respiratory enzymes. Proc. Royal Soc. Lon., B: Biol. Sci.. 1929, 104, 206-252.

9. $\quad$ Bierich, R., Rosenbohm, A. Cytochrome. Z. Physyol. Chem. 1929, 184, 246256.

10. Truman, J. W., de Vente, J., Ball, Eldon E. Nitric oxide - sensitive guanylate cyclase activity is associated with the maturational phase of neuronal development in insects. Development, Cambridge, U.K. 1996, 122, 3949-3958.

11. Rodgers, K. R. Heme-based sensors in biological systems. Curr. Opin. Chem. Biol. 1999, 3, 158-167.

12. Chan, M. K. Heme protein biosensors. J. Porph. Phthalocyan. 2000, 4, 358361.

13. Monson, E. K., Weinstein, M., Ditta, G. S., Helinski, D. R. The FixL protein of Rhizobium meliloti can be separated into a heme-binding oxygen-sensing 
domain and a functional C-terminal kinase domain. Proc. Nat.Acad. Sci. U.S.A. 1992, 89, 4280-4284.

14. Pellequer, J. L., Brudler, R., Getzoff, E. D. Biological sensors: more than one way to sense oxygen. Curr. Biol. 1999, 9, R416-418.

15. Perutz, M. F., Paoli, M., Lesk, A. M. Fix L, a haemoglobin that acts as an oxygen sensor: signalling mechanism and structural basis of its homology with PAS domains. Chem. Biol. 1999, 6, R291-297.

16. Delgado-Nixon, V.M., Gonzalez, G., Gilles-Gonzalez, M.A. Dos, a hemebinding PAS protein from Escherichia coli, is a direct oxygen sensor. Biochem. 2000, 39, 2685-2691.

17. Sasakura, Y., Yoshimura-Suzuki, T., Kurokawa, H., Shimizu, T. Structurefunction relationships of $E c D O S$ a heme-regulated phosphodiesterase from Escherichia coli. Acc. Chem. Res. 2006, 39, 37-43.

18. Dioum, E. M., Rutter, J., Tuckerman, J. R., Gonzalez, G., Gilles-Gonzalez, M. A., McKnight, S. L. NPAS2: a gas-responsive transcription factor. Science. 2002, 298, 2385-2387.

19. Gilles-Gonzalez, M. A. and Gonzalez, G. Signal transduction by hemecontaining PAS-domain proteins. J. App. Physiol. 2004, 96, 774-783.

20. Zhang, W. and Phillips, G. N. Jr. Structure of the oxygen sensorin Bacillus subtilis: signal transduction of chemotaxis by control of symmetry. Structure. 2003, 11, 1097-1110.

21. He, Y., Shelver, D., Kerby, R. L., Roberts, G. P. Characterization of a COresponsive transcriptional activator from Rhodospirillum rubrum. J. Biol. Chem. 1996, 271, 120-123.

22. Shelver, D., Kerby, R. L., He, Y., Roberts, G. P. CooA, a CO-sensing transcription factor from Rhodospirillum rubrum, is a CO-binding heme protein. Proc. Nat. Acad. Sci. U. S. A. 1997, 94, 11216-11220.

23. Klapper, M. H. and Hackett, D. P. The oxidative activity of horse-radish peroxidase. I. Oxidation of hydro- and naphthohydroquinones. J. Biol. Chem. 1963, 238, 3736-3742.

24. Renganathan, V., Miki, K., Gold, M. H. Haloperoxidase reactions catalyzed by lignin peroxidase, an extracellular enzyme from the basidiomycete Phanerochaete chrysosporium. Biochem. 1987, 26, 5127-5132. 
25. Wariishi, H., Valli, K., Renganathan, V., Gold, M. H. Thiol-mediated oxidation of nonphenolic lignin model compounds by manganese peroxidase of Phanerochaete chrysosporium. J. Biol. Chem.1989, 264, 14185-14191.

26. Daugherty, A., Dunn, J. L., Rateri, D. L., Heinecke, J. W. Myeloperoxidase, a catalyst for lipoprotein oxidation, is expressed in human atherosclerotic lesions. J. Clin. Investig. 1994, 94, 437-444.

27. Shaw, P. D. and Hager, L. P. Biological chlorination. VI. Chloroperoxidase: a component of the $\beta$-ketoadipate chlorinase system. J. Biol. Chem. 1961, 236, 1626-1630.

28. Hager, L. P., Morris, D. R., Brown, F. S., Eberwein, H. Chloroperoxidase. II. Utilization of halogen anions. J. Biol. Chem. 1966, 241, 1769-1777.

29. Beyer, W. F. J., Fridovich, I. Catalases - with and without heme. Basic Life Sci. 1988, 49, 651-661.

30. Deisseroth, A., Dounce, A. L. Catalase: physical and chemical properties, mechanism of catalysis, and physiological role. Physiol. Rev. 1970, 50, 319375.

31. Griffith, O. W., Stuehr, D. J. Nitric oxide synthases: properties and catalytic mechanism. Annu. Rev. Physiol. 1995, 57, 707-736.

32. Nakahara, K., Tanimoto, T., Hatano, K., Usuda, K., Shoun, H. Cytochrome P450 55A1 (P-450dNIR) acts as nitric oxide reductase employing NADH as the direct electron donor. J. Biol. Chem. 1993, 268, 8350-8355.

33. Mansuy, D. The great diversity of reactions catalyzed by cytochromes P450. Comp. Biochem. Physiol. Part C, Pharmacol. Toxicol. Endocrin. 1998, 121, 514.

34. Ortiz de Montellano, P. R., Choe, P., DePillis, G., Catalano, C. E. Structuremechanism relationships in hemoproteins. Oxygenations catalyzed by chloroperoxidase and horseradish peroxidase. J. Biol. Chem. 1987, 262, 1164111646.

35. Malatesta, F., Antonini, G., Sarti, P., Brunori, M. Structure and function of a molecular machine: cytochrome c oxidase. Biophys. Chem. 1995, 54, 1-33.

36. Silvestrini, M. C., Falcinelli, S., Ciabatti, I., Cutruzzolà, F., Brunori, M. Pseudomonas aeruginosa nitrite reductase (or cytochrome oxidase): An overview. Biochimie. 1994, 76, 641-654. 
37. Scott, R. A., and Mauk, A. G. Cytochrome c: A multidisciplinary approach. University Science Books. 1996.

38. Stroebel, D., Choquet, Y., Popot J. L., Picot, D. An atypical haem in the cytochrome $b_{6} f$ complex. Nature. 2003, 426, 413-418.

39. Puustinen, A. and Wikstrom, M. The heme groups of cytochrome o from Escherichia coli. Proc. Nat. Acad. Sci. U. S. A. 1991, 88, 6122-6126.

40. Zhang, Li (Editor). Heme biology: the secret life of heme in regulating diverse biological processes. World Sci. Pub. 2011, 167.

41. Landfried, D. A., Vuletich, D. A., Pond, M. P., Lecomte J. T. Structural and thermodynamic consequences of $\mathrm{b}$ heme binding for monomeric apoglobins and other apoproteins. Gene, 2007, 398, 12-28.

42. Schneider, S., Marles-Wright, J., Sharp, K. H., Paoli, M. Diversity and conservation of interactions for binding heme in b-type heme proteins. Nat. Prod. Rep. 2007, 24, 621-630.

43. Lu, Y., Berry, S. M., Pfister, T. D. Engineering novel metalloproteins: design of metal-binding sites into native protein scaffolds. Chem. Rev. 2001, 101, 3047-3080.

44. Chang, C. K., Traylor, T. G. Proximal base influence on the binding of oxygen and carbon monoxide to heme. J. Am. Chem. Soc. 1973, 95, 8477-8479.

45. Valentine, J. S., Sheridan, R. P., Allen, L. C., Kahn, P. C. Coupling between oxidation state and hydrogen bond conformation in heme proteins. Proc. Nat. Acad. Sci. USA 1979, 76, 1009-1013.

46. Ursini, F. and Bindoli, A. The role of selenium peroxidases in the protection against oxidative damage of membranes. Chem. Phys. Lipid. 1987, 44, 255276.

47. Zamocky, M., Hofbauer, S., Schaffner, I., Gasselhuber, B., Nicolussi, A., Soudi, M., Pirker, K. F., Furtmuller, P. G., Obinger, C. Independent evolution of four heme peroxidase superfamilies. Arch. Biochem. Biophys. 2015, 574, 108-119.

48. Torres E. and Ayala M. Biocatalysis based on heme peroxidases. Peroxidases as potential industrial biocatalysts. Springer Heidelberg Dordrecht Lon. NY. 2010.

49. Goblirsch, B., Kurker, R. C., Streit, B. R., Wilmot, C. M., DuBois, J. L. Chlorite 
dismutases, DyPs, and EfeB: 3 microbial heme enzyme families comprise the CDE structural superfamily. J. Mol. Biol. 2011, 408, 379-398.

50. Zamocky, M., Regelsberger, G., Jakopitsch, C., Obinger, C. The molecular peculiarities of catalase-peroxidases. FEBS let. 2001, 492, 177-182.

51. Altschul, A. M., Abrams, R., Hogness, T. R. Cytochrome c peroxidase. J. Biol. Chem. 1940, 136, 777-794.

52. Gerbling, K. P., Kelly, G. J., Fischer, K. H., Latzko, E. Partial purification and properties of soluble ascorbate peroxidases from pea leaves. J. Plant Physiol. 1984, 115, 59-67.

53. Camarero, S., Sarkar, S., Ruiz-Duenas, F. J., Martinez, M. J., Martinez, A. T. Description of a versatile peroxidase involved in the natural degradation of lignin that has both manganese peroxidase and lignin peroxidase substrate interaction sites. J. Biol. Chem. 1999, 274, 10324-10330.

54. Migler, R., De Chatelet, L. R. Human eosinophilic peroxidase: biochemical characterization. Biochem. Med. 1978, 19, 16-26.

55. Polis, B. D. and Shmukler, H. W. Crystalline lactoperoxidase. I. Isolation by displacement chromatography. II. Physicochemical and enzymic properties. $J$. Biol. Chem. 1953, 201, 475-500.

56. Alexander, N. M. A spectrophotometric assay for iodide oxidation by thyroid peroxidase. Anal. Biochem. 1962, 4, 341-345.

57. Streit, B. R. and DuBois, J. L. Chemical and Steady-State Kinetic Analyses of a Heterologously Expressed Heme Dependent Chlorite Dismutase. Biochemistry 2008, 47, 5271-5280.

58. Mlynek, G., Sjoeblom, B., Kostan, J., Fuereder, S., Maixner, F., Gysel, K., Furtmueller, P. G., Obinger, C., Wagner, M., Daims, H., Djinovic-Carugo, K. Unexpected diversity of chlorite dismutases: a catalytically efficient dimeric enzyme from Nitrobacter winogradskyi. J. Bacteriol. 2011, 193, 2408-2417.

59. Faraco, V., Piscitelli, A., Sannia, G., Giardina, P. Identification of a new member of the dye-decolorizing peroxidase family from Pleurotus ostreatus. World J. Microbiol. Biotechnol. 2007, 23, 889-893.

60. Zubieta, C., Krishna, S. S., Kapoor, M., Kozbial, P., McMullan, D., Axelrod, H. L., Miller, M. D., Abdubek, P., Ambing, E., Astakhova, T., et al. Crystal structures of two novel dye-decolorizing peroxidases reveal a $\beta$-barrel fold with a conserved heme-binding motif. Prot. Str. Func. Bioinform. 2007, 69, 223233. 
61. Liu, X., Du, Q., Wang, Z., Zhu, D., Huang, Y., Li, N., Wei, T., Xu, S., Gu, L. Crystal structure and biochemical features of EfeB/YcdB from Escherichia coli O157: ASP235 plays divergent roles in different enzyme-catalyzed processes. J. Biol. Chem. 2011, 286, 14922-14931.

62. Hofrichter, M., Kellner, H., Pecyna, M. J., Ullrich, R. Fungal unspecific peroxygenases: heme-thiolate proteins that combine peroxidase and cytochrome P450 properties. Adv. Exp. Med. Biol. 2015, 341-368.

63. Poulos, T. Peroxidase and heme thiolate enzymes. Handbook of porph sci. 2012, 19, 45-109.

64. Goodin, D. B., McRee, D. E. The Asp-His-Fe triad of cytochrome c peroxidase controls the reduction potential, electronic structure, and coupling of the tryptophan free radical to the heme (1CCA). Biochemistry 1993, 32, 3313-3324.

65. Blodig, W., Smith, A. T., Doyle, W. A., Piontek, K. Crystal structures of pristine and oxidatively processed lignin peroxidase expressed in Escherichia coli and of the W171F variant that eliminates the redox active tryptophan 171. Implications for the reaction mechanism (1B82). J. Mol. Biol. 2001, 305, 851861.

66. Gajhede, M., Schuller, D. J., Henriksen, A., Smith, A. T., Poulos, T. L. Crystal structure of horseradish peroxidase C at 2.15 A resolution (1ATJ). Nat. Struct. Biol. 1997, 4, 1032-1038.

67. de Visser, S. P. What external perturbations influence the electronic properties of catalase compound I? Inorg. Chem. 2006, 45, 9551-9557.

68. Fitzpatrick, F. A. Cyclooxygenase enzymes: regulation and function. Curr. Pharm. Des. 2004, 10, 577-588.

69. Sheikh, I. A., Ethayathulla, A. S., Singh, A. K., Singh, N., Sharma, S., Singh, T. P. Crystal structure of Buffalo lactoperoxidase at 2.75A resolution. P. D. B. (2GJM), 2006.

70. Yoshida, T., Tsuge, H., Konno, H., Hisabori, T., Sugano, Y. The catalytic mechanism of dye-decolorizing peroxidase DyP may require the swinging movement of an aspartic acid residue (3AFV). FEBS J. 2011, 278, 2387-2394.

71. Sundaramoorthy, M., Terner, J., Poulos, T. The crystal structure of chloroperoxidase: a heme peroxidase-cytochrome P450 functional hybrid. Structure 1995, 3, 1367-1377. 
72. Sugano, Y., Muramatsu, R., Ichiyanagi, A., Sato, T., Shoda, M. DyP, a unique dye-decolorizing peroxidase, represents a novel heme peroxidase family: ASP171 replaces the distal histidine of classical peroxidases. J. Biol. Chem. 2007, 282, 36652-36658.

73. Kim, S. J. and Shoda, S. Purification and characterization of a novel peroxidase from Geotrichum candidum Dec 1 involved in decolorization of dyes. Appl. Environ. Microbiol. 1999, 65, 1029-1035.

74. Johjima, T., Ohkuma, M., Kudo T. Isolation and cDNA cloning of novel hydrogen peroxide-dependent phenol oxidase from the basidiomycete Termitomyces albuminosus. Appl. Microbiol. Biotechnol. 2003, 61, 220-225.

75. Kim S. J., Ishikawa K., Hirai M., Shoda M. Characteristics of a newly isolated fungus, Geotrichum candidum Dec 1, which decolorizes various dyes. J. Ferm. Bioeng. 1995, 79, 601-607.

76. Liers, C., Bobeth, C., Pecyna, M., Ullrich, R., Hofrichter, M. DyP-like peroxidases of the jelly fungus Auricularia auricula-judae oxidize nonphenolic lignin model compounds and high-redox potential dyes. Appl. Microbio.l Biotechnol. 2010, 85(6):1869-1879.

77. Yoshida T., Tsuge H., Hisabori T., Sugano, Y. Crystal structures of dyedecolorizing peroxidase with ascorbic acid and 2,6-dimethoxyphenol. FEBS Let. 2012, 586, 4351-4356.

78. Linde, D., Coscolín, C., Liers, C., Hofrichter, M., Martínez, A. T. , RuizDueñas, F.J. Heterologous expression and physicochemical characterization of a fungal dye-decolorizing peroxidase from Auricularia auricula-judae. Prot. Expr. Purif. 2014, 103, 28-37.

79. Linde, D. , Ruiz-Dueñas, F. J.,Fernández-Fueyo, E., Guallar, V., Hammel, K. E., Pogni , R., Martínez A. T. Basidiomycete DyPs: genomic diversity, structural-functional aspects, reaction mechanism and environmental significance. Arch. Biochem. Biophys. 2015, 574, 66-74.

80. Anh, D. H. Ullrich, R., benndorf, D., Svatos, A., Muck, A., Hofrichter M. The coprophilous mushroom Coprinus radians secrets a haloperoxidase that catalyzes aromatic peroxygenation. Appl. Environ. Microbiol. 2007, 73, 54775485.

81. Pecyna, M. J., Ullrich, R., Bittner, B., Clemens, A., Scheibner, K., Schubert, R., Hofrichter, M. Molecular characterization of aromatic peroxygenases from Agrocybe aegerite. Appl. Microbiol. Biotechnol. 2009, 84, 885-897. 
82. Grobe, G., Ullrich, R., Pecyna, M. J., Kapturska, D., Friedrich, S., Hofrivhter, M., Scheibner, K. High-yield production of aromatic peroxygenases by the agaric fungus Marasmius rotula. AMB Express. 2011, 1, 31.

83. Ullrich, R. and Hofrichter, M. Enzymatic hydroxylation of aromatic compounds. Cell. Mol. Life Sci. 2007, 64, 271-293.

84. Aranda, E., Kinne, M., Kluge, M., Ullrich, R., Hofrichter, M. Conversion of dibenzothiophene by the mushrooms Agrocybe aegerita and Coprinellus radians and their extracellular peroxygenases. Appl. Microbiol. Biotechnol. 2009, 82, 1057-1066.

85. Aranda, E., Ullrich, R., Hofrichter, M. Conversion of polycyclic aromatic hydrocarbons, methyl naphthalenes and dibenzofuran by two fungal peroxygenases. Biodegradation 2009, 21, 267-281.

86. Poraj-Kobielska, M., Kinne, M., Ullrich, R., Scheibner, K., Kayser, G., Hammel, K. E., Hofrichter, M. Preparation of human drug metabolites using fungal peroxygenases. Biochem. Pharmacol. 2011, 82, 789-796.

87. Wanga, X., Ullrich, R., Hofrichter, M., Groves, J. T. Heme-thiolate ferryl of aromatic peroxygenase is basic and reactive. PNAS 2015, 112, 3686-3691.

88. Morozov, A. N., Pardillo, A. D., Chatfield, D. C. Chloroperoxidase-catalyzed epoxidation of cis- $\beta$-methylstyrene: $\mathrm{NH}-\mathrm{S}$ hydrogen bonds and proximal helix dipole change the catalytic mechanism and significantly lower the reaction barrier. J. Phys. Chem. B, 2015, 119, 14350-14363.

89. Piontek, K., Strittmatter, E., Ullrich, R., Grobe, G., Pecyna, M. J., Kluge, M., Scheibner, K., Hofrichter, M., Plattner, D. A. Structural Basis of Substrate Conversion in a New Aromatic Peroxygenase: P450 Functionality with Benefits (2YOR). J.Biol.Chem. 2013, 288, 34767-34776.

90. Williams, P. A., Cosme, J., Vinkovic, D. M., Ward, A., Angove, H. C., Day, P. J., Vonrhein, C., Tickle, I. J., Jhoti, H. Crystal Structures of Human Cytochrome P450 3A4 Bound to Metyrapone and Progesterone. Science 2004, 305, 683686.

91. Isin, E. M. and Guengerich, F. P. Complex reactions catalyzed by cytochrome P450 enzymes. Biochim. Biophys. Act. 2007, 1770, 314-329.

92. Matsunaga, T., Tanaka, H., Komura, A., Watanabe, K., Yamamoto, I., Yoshimura, $\mathrm{H}$. Microsomal alcohol oxygenase: purification and characterization of a cytochrome P450 responsible for oxidation of 7-hydroxy$\Delta 8$-tetrahydrocannabinol to 7-oxo- $\Delta 8$-tetrahydrocannabinol in guinea pig liver. 
Arch. Biochem. Biophys. 1997, 348, 56-64.

93. Matsunaga, I., Ueda, A., Fujiwara, N., Sumimoto, T., Ichihara, K. Characterization of the ybdT gene product of Bacillus subtilis: novel fatty acid $\beta$-hydroxylating cytochrome P450. Lipids 1999, 34, 841-846.

94. Hofrichter, M. and Ullrich, R. Heme-thiolate haloperoxidases: versatile biocatalysts with biotechnological and environmental significance. Appl. Microbiol. Biotechnol. 2006, 71, 276-288.

95. Morris, D. R. and Hager, L. P. Chloroperoxidase. I. Isolation and properties of the crystalline glycoprotein. J. Biol. Chem. 1966, 241, 1763-1768.

96. Kenigsberg P, Fang G, Hager L. Posttranslational modifications of chloroperoxidase from Caldariomyces fumago. Arch. Biochem. Biophys. 1987, 254, 409-415.

97. Conesa, A., Weelink, G., van den Hondel, C. A., Punt, P. J. C-terminal propeptide of the Caldariomyces fumago chloroperoxidase: an intramolecular chaperone? FEBS Lett. 2001, 503, 117-120.

98. Poulos, T. L., Finzel, B. C., Howard, A. J. High-resolution crystal structure of cytochrome P450cam. J. Mol. Biol. 1987, 195, 687-700.

99. Hol, W. G. J., van Duijnen, P. T., Berendsen, H. J. C. The $\alpha$-helix dipole and the properties of proteins. Nature 1978, 273, 443-446.

100. Lockhart, D. J. and Kim, P. S. Internal Stark Effect measurement of the electric field at the amino terminus of an $\alpha$-helix. Science 1992, 257, 947-951.

101. Yoshioka, S., Tosha, T., Takahashi, S., Ishimori, K., Hori, H., Morishima, I. Roles of the Proximal Hydrogen Bonding Network in Cytochrome P450camCatalyzed Oxygenation. J. Am. Chem. Soc. 2002, 124, 14571-14579.

102. Galinato,M. G. I., Spolitak, T., Ballou D. P., Lehnert, N. Elucidating the role of the proximal cysteine hydrogen bonding network in ferric cytochrome P450cam and corresponding mutants using magnetic circular dichroism spectroscopy. Biochem. 2011, 50, 1053-1069.

103. Kuehnel, K., Blankenfeldt, W.,Terner, J., Schlichting, I. Crystal structures of chloroperoxidase with its bound substrates and complexed with formate, acetate, and nitrate. J. Biol. Chem. 2006, 281, 23990-23998. 
104. Hager, L. P., Doubek, D. L., Silverstein, R. M., Hargis, J. H., Martin, J. C. Chloroperoxidase. IX. The structure of compound I. J. Am. Chem. Soc. 1972, 94, 4364-4366.

105. Chen, H., Hirao, H., Derat, E., Schlichting, I., Shaik, S. Quantum mechanical/molecular mechanical study on the mechanisms of compound I formation in the catalytic cycle of chloroperoxidase: an overview on heme enzymes. J. Phys. Chem. B. 2008, 112, 9490-9500.

106. Choudhury, K.., Sundaramoorthy, M., Hickman, A., Yonetani, T., Woehl, E., Dunn, M. F., Poulos, T. L. Role of the proximal ligand in peroxidase catalysis. Crystallographic, kinetic, and spectral studies of cytochrome c peroxidase proximal ligand mutants. J. Biol. Chem. 1994, 269, 20239-20249.

107. Green, M. T., Dawson, J. H., Gray, H. B. Oxoiron(IV) in chloroperoxidase Compound II is basic: implications for P450 chemistry. Science 2004, 304, 1653-1656.

108. Lai, W., Chen, H., Shaik, S. What kinds of ferryl species exist for compound II of chloroperoxidase? A dialog of theory with experiment. J. Phys. Chem. B. 2009, 113, 7912-7917.

109. Wagenknecht, H. A. and Woggon, W. D. Identification of intermediates in the catalytic cycle of chloroperoxidase. Chem. Biol. 1997, 4, 367-372.

110. Woggon, W. D., Wagenknecht, H. A., Claude, C. Synthetic active site analogues of heme-thiolate proteins: Characterization and identification of intermediates of the catalytic cycles of cytochrome P450cam and chloroperoxidase. J. Inorg. Biochem. 2001, 83, 289-300.

111. Doerge, D. R. and Corbett, M. D. Peroxygenation mechanism for chloroperoxidase-catalyzed N-oxidation of arylamines. Chem. Res. Toxicol. 1991, 4, 556-560.

112. Manoj, K. M. and Hager, L. P. Chloroperoxidase, a Janus enzyme. Biochemistry 2008, 47, 2997-3003.

113. Hofrichter, M., Ullrich, R., Pecyna, M. J., Liers, C., Lundell, T. New and classic families of secreted fungal heme peroxidases. Appl. Microbiol. Biotechnol. 2010, 87, 871-897.

114. Corbett, M. D, Chipko, B. R., Baden, D. G. Chloroperoxidase-catalyzed oxidation of 4-chloroaniline to 4-chloronitrosobenzene. Biochem. J. 1978, 175, 353-360. 
115. Geigert, J., Dalietos, D. J., Neidleman, S. L., Lee, T. D., Wadsworth, J. Peroxide oxidation of primary alcohols to aldehydes by chloroperoxidase catalysis. Biochem. Biophys. Res. Commun. 1983, 114, 1104-1108.

116. Kiljunen, E. and Kanerva, L. T. Chloroperoxidase-catalysed oxidation of alcohols to aldehydes. J. Mol. Cat. B-Enz. 2000, 9, 163-172.

117. Zaks, A. and Dodds, D. R. Chloroperoxidase-catalyzed asymmetric oxidations: substrate specificity and mechanistic study. J. Am. Chem. Soc. 1995, 117, 10419-10424.

118. Geigert, J., DeWitt, S. K., Neidleman, S. L., Lee, G., Dalietos, D. J., Moreland, M. DMSO is a substrate for chloroperoxidase. Biochem. Biophys. Res. Commun. 1983, 116, 82-85.

119. Lakner, F., Cain, K., Hager, L. Enantioselective epoxidation of omega-bromo2-methyl-1-alkenes catalyzed by chloroperoxidase. Effect of chain length on selectivity and efficiency. J. Am. Chem. Soc. 1997, 119, 443-444.

120. Hu, S. and Hager, L. Unusual propargylic oxidations catalyzed by chloroperoxidase. Biochem. Biophys. Res. Commun. 1998, 253, 544-546.

121. Hu, S. and Hager, L. Asymmetric epoxidation of functionalized cis-olefins catalyzed by chloroperoxidase. Tetrahedron Lett. 1999, 40, 1641-1644.

122. Hu, S. and Hager, L. Highly enantioselective propargylic hydroxylations catalyzed by chloroperoxidase. J. Am. Chem. Soc. 1999, 121, 872-873.

123. Bougioukou, D. J. and Smonou, I. Chloroperoxidase-catalyzed oxidation of conjugated dienoic esters. Tetrahedron Lett. 2002, 43, 339-342.

124. Miller, V. P., Tschirret-Guth, R. A., Ortiz de Montellano, P. R. Chloroperoxidase-catalyzed benzylic hydroxylation. Arch. Biochem. Biophys. 1995, 319, 333-340.

125. van Deurzen, M. P. J., Remkes, I. J., van Rantwijk, F., Sheldon, R. A. Chloroperoxidase catalyzed oxidations in $t$-butyl alcohol/water mixtures. $J$. Mol. Cat. A-Chem. 1997, 117, 329-337.

126. van de Velde, F., Bakker, M., van Rantwijk, F., Sheldon, R. Chloroperoxidasecatalyzed enantioselective oxidations in hydrophobic organic media. Biotechnol. Bioeng. 2001, 72, 523-529.

127. Lambeir, A. M., Dunford, H. B., Pickard, M. A. Kinetics of the oxidation of ascorbic acid, ferrocyanide and p-phenolsulfonic acid by chloroperoxidase compounds I and II. Eur. J. Biochem. 1987, 163, 123-127. 
128. Conesa, A., Punt, P., van den Hondel, C. Fungal peroxidases: molecular aspects and applications. J. Biotechnol. 2002, 93, 143-158.

129. Gallati, H. Horseradish-peroxidase - study of the kinetics and the determination of optimal reaction conditions, using hydrogen-peroxide and 2,2-azino-bis-3ethylbenzthiazoline-6-sulfonic acid (ABTS) as substrates. J. Clin. Chem. Clin. Biochem. 1979, 17, 1-7.

130. Doerge, D. R., Divi, R. L., Churchwell, M. I. Identification of colored guaiacol oxidation product produced by peroxidases. Anal. Biochem. 1997, 250, 10-17.

131. Casella, L., Poli, S., Gulloti, M., Selvaggini, C., Beringhelli, T., Marchesini, A. The chloroperoxidase-catalyzed oxidation of phenols. Mechanism, selectivity, and characterization of enzyme-substrate complexes. Biochem. 1994, 33, 63776386.

132. Thomas, J. A., Morris, D. R., and Hager, L. P. Chloroperoxidase VII: Classical peroxidatic, catalatic and halogenating forms of the enzyme. J. Biol. Chem. 1970, 245, 3129-3134.

133. Reichman, M. and Ritter, H. Synthesis of phenol polymers using peroxidases. Adv. Polym. Sci. 2006, 194, 1-49.

134. Ujama, K. and Kobayashi, S. Enzyme-catalyzed polymerization to functional polymers. J. Mol. Catal. B: Enzym. 2002, 19-20, 117-127.

135. Bodalo, A., Gomez, J. L., Gomez, E., Bastyda, J., Malimo, M.F. Comparison of commercial peroxidases for removing phenol from water solutions. Chemosphere 2006, 63, 626-632.

136. Cheng, X., Jia, R., Li, P., Tu, S., Zhu, Q., Tang, W., Li, X. Purification of a new manganese peroxidase of the white-rot fungus Schizophyllum sp.F17, and decolorization of azo dyes by the enzyme. Enz. Microb. Technol. 2007, 41, 258264.

137. Osborne, R., Raner, G., Hager, L., Dawson, J. C. fumago chloroperoxidase is also a dehaloperoxidase: oxidative dehalogenation of halophenols. J. Am. Chem. Soc. 2006, 128, 1036-1037.

138. Osborne, R. L., Coggins, M. K., Terner, J., Dawson, J. H. Caldariomyces fumago chloroperoxidase catalyzes the oxidative dehalogenation of chlorophenols by a mechanism involving two one-electron steps. J. Am. Chem. Soc. 2007, 129, 14838-14839. 
139. Murphy, C. D. Fluorophenol oxidation by a fungal chloroperoxidase. Biotechnol. Lett. 2007, 29, 45-49.

140. Diaz-Diaz, G., Blanco-Lopez, M. C., Lobo-Castanon, M. J., Miranda-Ordieres, A. J., Tunon-Blanco, P. Kinetic study of the oxidative dehalogenation of 2,4,6trichlorophenol catalyzed by chloroperoxidase. J. Mol. Cat. B Enz. 2010, 66, 332-336.

141. Manoj, K. M. Chlorinations catalyzed by chloroperoxidase occur via diffusible intermediate(s) and the reaction components play multiple roles in the overall process. Biochim. Biophys. Acta 2006, 1764, 1325-1339.

142. Casella, L., Gulloti, M., Ghezzi, R., Poli, S., Beringhelli, T., Colonna, S., Carrea, G. Mechanism of enantioselective oxygenation of sulfides catalyzed by chloroperoxidase and horseradish peroxidase. Spectral studies and characterization of enzyme-substrate complexes. Biochemistry 1992, 31, 94519459.

143. Libby, R., Thomas, J., Kaiser, L., Hager, L. Chloroperoxidase halogenation reactions - chemical versus enzymic halogenating intermediates. J. Biol. Chem. 1982, 257, 5030-5037.

144. Taurog, A. and Howells, E. M. Enzymatic iodination of tyrosine and thyroglobulin with chloroperoxidase. J. Biol. Chem. 1966, 241, 1329-1339.

145. Vazquez-Duhalt,R., Ayala, M., Marquez-Rocha, F. Biocatalytic chlorination of aromatic hydrocarbons by chloroperoxidase of Caldariomyces fumago. Phytochem. 2001, 58, 929-933.

146. Ramakrishnan, K., Oppenhuizen, M., Saunder, S. S., Fisher, J. Stereoselectivity of chloroperoxidase-dependent halogenation. Biochemistry 1983, 22, 32713277.

147. Allain, E. J., Hager, L. P., Deng, L., Jacobsen, E. N. Highly enantioselective epoxidation of disubstituted alkenes with hydrogen peroxide catalyzed by chloroperoxidase. J. Am. Chem. Soc. 1993, 115, 4415-4416.

148. Colonna, S., Gaggero, N., Casella, L., Garrea, G., Pasta, P. Chloroperoxidase and hydrogen peroxide: an efficient system for enzymatic enantioselective sulfoxidations. Tetrahedron-Asymm. 1992, 3, 95-106.

149. van Rantwijk, F. and Sheldon, R. A. Selective oxygen transfer catalyzed by heme peroxidases: synthetic and mechanistic aspects. Curr. Opin. Biotechnol. 2000, 11, 554-564. 
150. Dexter, A. F., Lakner, F. J., Campbell, R. A., Hager, L. P. Highly enantioselective epoxidation of 1,1-disubstituted alkenes catalyzed by chloroperoxidase. J. Am. Chem. Soc. 1995, 117, 6412-6413.

151. Besse, P. and Veschambre, H. Chemical and biological synthesis of chiral epoxides. Tetrahedron 1994, 50, 8885-8927.

152. Ayala, M., Robledo, N., Lopez-Munguia, A., Vazquez-Duhalt, R. Substrate specificity and ionization potential in chloroperoxidase-catalyzed oxidation of diesel fuel. Environ. Sci. Technol. 2000, 34, 2804-2809.

153. Manoj, K. M. and Hager, L. P. Utilization of peroxide and its relevance in oxygen insertion reactions catalyzed by chloroperoxidase. Biochim. Biophys. Acta 2001, 1547, 408-417.

154. Lakner, F. and Hager, L. Chloroperoxidase as enantioselective epoxidation catalyst: An efficient synthesis of (R)-(-)-mevalonolactone. J. Org. Chem. 1996, 61, 3923-3925.

155. Lakner, F. and Hager, L. Chloroperoxidase-mediated asymmetric epoxidation. Synthesis of (R)-dimethyl-2-methylaziridine-1,2-dicarboxylate - a potential alpha-methylamino acid synthon. Tetrahedron-Asymm. 1997, 8, 3547-3550.

156. Kobayashi, S., Nakano, M., Goto, T., Kimura, T., Schaap, A. P. An evidence of the peroxidase-dependent oxygen transfer from hydrogen peroxide to sulfides. Biochem. Biophys. Res. Commun. 1986, 135, 166-171.

157. Colonna, S., Gaggero, N., Manfredi, A., Casella, L., Gullotti, M., Garrea, G., Pasta, P. Enantioselective oxidations of sulfides catalyzed by chloroperoxidase. Biochemistry 1990, 29, 10465-10468.

158. Allenmark, S. G. and Andersson, M. A. Chloroperoxidase-catalyzed asymmetric synthesis of a series of aromatic cyclic sulfoxides. TetrahedronAsymm. 1996, 7, 1089-1094.

159. Pasta, P., Carrea, G., Monzani, E., Gaggero, N., Colonna, S. Chloroperoxidasecatalyzed enantioselective oxidation of methyl phenyl sulfide with dihydroxyfumaric acid/oxygen or ascorbic acid/oxygen as oxidants. Biotechnol. Bioengin. 1999, 62, 489-493.

160. Vargas, R. R., Bechara, E. J. H., Marzorati, L., Wladislaw, B. Asymmetric sulfoxidation of a beta-carbonyl sulfide series by chloroperoxidase. Tetrahedron-Asymm. 1999, 10, 3219-3227. 
161. Ayala, M., Pickard, M. A., Vazquez-Duhalt, R. Fungal enzymes for environmental purposes, a molecular biology challenge. J. Mol. Microbiol. Biotechnol. 2008, 15:172-180.

162. Gao, F., Wang, L., Liu, Y., Wang, S., Jiang, Y., Hu, M., Li, S., Zhai, Q. Enzymatic synthesis of $(R)$-modafinil by chloroperoxidase-catalyzed enantioselective sulfoxidation of 2-(diphenylmethylthio) acetamide. Biochem. Engineer. J. 2015, 93, 243-249.

163. Sanfilippo, C. and Nicolosi, G. Catalytic behaviour of chloroperoxidase from Caldariomyces fumago in the oxidation of cyclic conjugated dienes. Tetrahedron-Asymm. 2002, 13, 1889-1892.

164. Sanfilippo, C., Patti, A., Nicolosi, G. Asymmetric oxidation of 1,3cyclohexadiene catalysed by chloroperoxidase from Caldariomyces fumago. Tetrahedron-Asymm. 2000, 11, 3269-3272.

165. Aguila, S., Vazquez-Duhalt, R., Tinoco, R., Rivera, M., Pecchi, G., Alderete, J. B. Stereoselective oxidation of R-(+)-limonene by chloroperoxidase from Caldariomyces fumago. Green Chem. 2008, 10, 647-653.

166. Liu, Y., Wang, Y., Jiang, Y., Hu, M., Li, S., Zhai, Q. Biocatalytic synthesis of C3 chiral building blocks by chloroperoxidase-catalyzed enantioselective halohydroxylation and epoxidation in the presence of ionic liquids. Biotechnol. Prog. 2015, 31, 724-729.

167. Kaup, B., Piantini, U., Wuest, M., Schrader, J. Monoterpenes as novel substrates for oxidation and halo-hydroxylation with chloroperoxidase from Caldariomyces fumago. Appl. Microbiol. Biotechnol. 2007, 73, 1087-1096.

168. Fu, H., Kondo, H., Ichikawa, Y., Look, G. C., Wong, C. H. Chloroperoxidasecatalyzed asymmetric synthesis: enantioselective reactions of chiral hydroperoxides with sulfides and bromohydration of glycals. J. Org. Chem. 1992, 57, 7265-7270.

169. Corbett, M. D. and Chipko, B. R. Peroxide oxidation of indole to oxindole by chloroperoxidase catalysis. Biochem. J. 1979, 183, 269-276.

170. Zhang, R., He, Q., Chatfield, D., Wang, X. Paramagnetic nuclear magnetic resonance relaxation and molecular mechanics studies of the chloroperoxidaseindole complex: insights into the mechanism of chloroperoxidase-catalyzed regioselective oxidation of indole. Biochemistry 2013, 52, 3688-3701.

171. Corbett, M. D., Baden, D. G., Chipko, B. R. Arylamine oxidations by chloroperoxidase. Bioorg. Chem. 1979, 8, 91-95. 
172. Hollenberg, P. F. and Hager, L. P. P-450 nature of the carbon monoxide complex of ferrous chloroperoxidase. J. Biol. Chem. 1973, 248, 2630-2633.

173. Sono, M. and Dawson, J. H. Formation of low spin complexes of ferric cytochrome P-450-CAM with anionic ligands. Spin state and ligand affinity comparison to myoglobin. J. Biol. Chem.1982, 257, 5496-5502.

174. Sono, M., Dawson, J., Hall, K., Hager, L. Ligand and halide binding properties of chloroperoxidase - peroxidase-type active site heme environment with cytochrome P-450 type endogenous axial ligand and spectroscopic properties. Biochem (NY) 1986, 25, 347-356.

175. Ogunmola, G. B., Zipp, A., Chen, F., Kauzmann, W. Effects of pressure on visible spectra of complexes of myoglobin, hemoglobin, cytochrome c, and horse radish peroxidase. Proc. Natl. Acad. Sci. U. S. A. 1977, 74, 1-4.

176. Sundaramoorthy, M., Terner, J., Poulos, T. Stereochemistry of the chloroperoxidase active site: crystallographic and molecular-modeling studies. Chem. Biol. 1998, 5, 461-473.

177. Sawamura, T., Tanaka, T., Ishige, H., Iizuka, M., Murayama, Y., Otsuji, E., Ohkubo, A., Ogura, S. I., Yuasa, H. The effect of coating on the affinity of lanthanide nanoparticles to MKN45 and HeLa cancer cells and improvement in photodynamic therapy efficiency. Int. J. Mol. Sci. 2015, 16, 22415-22424.

178. Gouterman, M., Wagniere, G. H., Snyder, L. C. Spectra of porphyrins: part II. Four orbital model. J. Mol. Spectrosc. 1963, 11, 108-127.

179. Perrin, M. H., Gouterman, M., Perrin, C. L. Vibronic coupling. VI. Vibronic borrowing in cyclic polyenes and porphyrin. J. Chem. Phys. 1969, 50, 41374150 .

180. Marsh, D. F. and Mink, L. M. Microscale synthesis and electronic absorption spectroscopy of tetraphenylporphyrin H2(TPP) and metalloporphyrins ZnII(TPP) and Ni II (TPP), J. Chem. Ed., 1996, 73, 1181.

181. Spiro, T. G. and Strekas, T. C. Resonance Raman spectra of heme proteins. Effects of oxidation and spin state. J.A.C.S. 1974, 96, 338-345.

182. Spiro, T. G. Resonance Raman spectroscopy as a probe of heme protein structure and dynamics. Adv. Prot. Chem. 1985, 37, 111-159.

183. Wang, X., Tachikawa, H., Yi, X., Manoj, K., Hager, L. Two-dimensional NMR 
study of the heme active site structure of chloroperoxidase. J. Biol. Chem. 2003, $278,7765-7774$.

184. Provencher, S.W. and Glockner, J. Estimation of globular protein secondary structure from circular dichroism. Biochemistry 1981, 20, 33-37.

185. van Stokkum, I. H. M., Spoelder, H. J. W., Bloemendal, M., Van Grondelle, R., and Groen, F. C. A. Estimation of protein secondary structure and error analysis from CD spectra. Anal. Biochemistry 1990, 191, 110-118.

186. Sreerema, N. and Woody, R.W. A self-consistent method for the analysis of protein secondary structure from circular dichroism. Anal. Biochem. 1993, 209, 32-44.

187. Sreerema, N., Venyaminov, S.Y., and Woody, R.W. Estimation of the number of helical and strand segments in proteins using CD spectroscopy. Protein Sci. 1999, 8, 370-380.

188. Compton, L. A. and Johnson, W. C., Jr. Analysis of protein circular dichroism spectra for secondary structure using a simple matrix multiplication. Anal. Biochem. 1986, 155, 155-167.

189. Manavalan, P. and Johnson, W.C., Jr. Variable selection method improves the prediction of protein secondary structure from circular dichroism spectra. Anal. Biochem. 1987, 167, 76-85.

190. Sreerama, N. and Woody, R.W. Estimation of protein secondary structure from CD spectra: Comparison of CONTIN, SELCON and CDSSTR methods with an expanded reference set. Anal. Biochem. 2000, 287, 252-260.

191. Andrade, M. A., Chacón, P., Merelo, J. J., Morán, F. Evaluation of secondary structure of proteins from UV circular dichroism using an unsupervised learning neural network. Prot. Engineer. 1993, 6, 383-390.

192. Munro, A. W., Lindsay, J. G., Coggins, J.R., Kelly, S. M., Price, N. C. Structural and enzymological analysis of the interaction of isolated domains of cytochrome P-450 BM3. FEBS Lett. 1994, 343, 70-74.

193. Venyaminov, S. Y. and Vassilenko, K. S. Determination of protein tertiary structure class from circular dichroism spectra. Anal. Biochem. 1994, 222, 176184.

194. Watanabe, Y. Construction of heme enzymes: four approaches. Curr. Opin. Chem. Biol. 2002, 6, 208-216.

195. Ozaki, S. I., Roach, M. P., Matsui, T., Watanabe, Y. Investigations of the roles 
of distal heme environment and the proximal heme iron ligand in peroxide activation by heme enzymes via molecular engineering of myoglobin. Acc. Chem. Res. 2001, 34, 818-825.

196. Adachi, S., Nagano, S., Ishimori, K., Watanabe, Y., Morishima, I., Egawa, T., Kitagawa, T., Makino, R. Roles of proximal ligand in heme proteins: replacement of proximal histidine of human myoglobin with cysteine and tyrosine by site-directed mutagenesis as models for P-450, chloroperoxidase, and catalase. Biochem. 1993, 32, 241-252.

197. Savenkova, M. I., Kuo, J. M., Ortiz de Montellano, P. R. Improvement of peroxygenase activity by relocation of a catalytic histidine within the active site of horseradish peroxidase. Biochem. 1998, 37, 10828-10836.

198. Tanaka, M., Ishimori, K., Mukai, M., Kitagawa, T., Morishima, I. Catalytic activities and structural properties of horseradish peroxidase distal His $42 \rightarrow$ Glu or Gln mutant. Biochemistry 1997, 36, 9889-9898.

199. Newmyer, S. L. and Ortiz de Montellano, P. R. Horseradish peroxidase His$42 \rightarrow$ Ala, His- $42 \rightarrow$ Val, and Phe- $41 \rightarrow$ Ala mutants. Histidine catalysis and control of substarte acces to the heme iron. J. Biol. Chem. 1995, 270, 1943019438.

200. Nagano, S., Tanaka, M., Ishimori, K., Watanabe, Y., Morishima, I. Catalytic roles of the distal site asparagine-histidine couple in peroxidases. Biochem. 1996, 35, 14251-14258.

201. Foshay, M. C. Vitello, L. B., Erman, J. E. Relocation of the distal histidine in cytochrome $c$ peroxidase: properties of $\mathrm{CcP}(\mathrm{W} 51 \mathrm{H}), \mathrm{CcP}(\mathrm{W} 51 \mathrm{H} / \mathrm{H} 52 \mathrm{~W})$, and CcP(W51H/H52L). Biochemistry 2009, 48, 5417-5425.

202. Foshay, M. C., Vitello, L. B., Erman, J. E. Effect of alternative distal residues on the reactivity of cytochrome c peroxidase: properties of CcP mutants H52D, H52E, H52N, and H52Q. Biochim. Biophys. Acta, 2011, 1814, 525-535.

203. England, P. A., Harford-Cross, C. F., Stevenson, J. A., Rouch, D. A., Wong, L. L. The oxidation of naphthalene and pyrene by cytochrome P450cam. FEBS Lett. 1998, 424, 271-274.

204. Aldag, C., Gromov, I. A., Garcia-Rubio, I., von Koenig, K., Schlichting, I., Juan, B., Hilvert, D. Probing the role of the proximal heme ligand in cytochrome P450cam by recombinant incorporation of selenocysteine. Proc. Nat. Acad. Sci. U. S. A. 2009, 106, 5481-5486. 
205. Behera, R. K., Goyal, S., Mazumdar, S. Modification of the heme active site to increase the peroxidase activity of thermophilic cytochrome P450: a rational approach. J. Inorg. Biochem. 2010, 104, 1185-1194.

206. Yi, X., Conesa, A., Punt, P., Hager, L. Examining the role of glutamic acid 183 in chloroperoxidase catalysis. J. Biol. Chem. 2003, 278, 13855-13859.

207. Li, H. and Poulos, T. L. Structural variation in heme enzymes: a comparative analysis of peroxidase and P450 crystal structures. Structure 1994, 2, 461-464.

208. Collman, J. P., brauman, J. I., Doxsee, K. M. Carbon monoxide binding to iron porphyrins. Proc. Natl. Acad. Sci. U. S. A. 1979, 76, 6035-6039.

209. Farhangrazi, Z. S, Sinclair, R., Yamazaki, I., Powers, L. S. Haloperoxidase activity of Phanerochaete chrysosporium lignin peroxidases H2 and H8. Biochemistry 1992, 31, 10763-10768.

210. Taurog, A. and Dorris, M. L. Peroxidase-catalyzed bromination of tyrosine, thyroglobulin, and bovine serum albumin: comparison of thyroid peroxidase and lactoperoxidase. Arch. Biochem. Biophys. 1991, 287, 288-296.

211. Matsui, T., Nagano, S., Ishimori, K., Watanabe, Y., Morishima, I. Preparation and reactions of myoglobin mutants bearing both proximal cysteine ligand and hydrophobic distal cavity: protein models for the active site of P450. Biochem. 1996, 35, 13118-13124.

212. Vatsis, K. P., Peng, H. M., Coon M. J. Abolition of oxygenase function, retention of NADPH oxidase activity, and emergence of peroxidase activity upon replacement of the axial cysteine-436 ligand by histidine in cytochrome P450 2B4. Arch. Biochem. Biophys. 2005, 434, 128-138.

213. Yoshioka, S., Takahashi, S., Hori, H., Ishimori, K., Morishima, I. Proximal cysteine residue is essential for the enzymatic activities of cytochrome P450cam. Eur. J. Biochem. / FEBS 2001, 268(2), 252-259.

214. Auclair, K., Moeenne-Loccoz, P., Ortiz de Montellano, P. R. Roles of the proximal heme thiolate ligand in cytochrome P450cam. J. Am. Chem. Soc. 2001, 123, 4877-4885.

215. Yi, X., Mroczko, M., Manoj, K. M., Wang, X., Hager, L. P. Replacement of the proximal heme thiolate ligand in chloroperoxidase with a histidine residue. Proc. Nat. Acad. Sci. U. S. A. 1999, 96, 12412-12417.

216. Punt, P. J. and van den Hondel, C. A. M. J. J. Transformation of filamentous fungi based on hygromycin B and phleomycin resistance markers. Meth. 
Enzymol. 1992, 216, 447-457.

217. Hutner, S. H., Provasoli, L., Schatz, A., Haskins, C. P. Some approaches to the study of the role of metals in the metabolism of microorganisms. Proc. Am. Phil. Soc. 1950, 94, 152-170.

218. Whitmore, L., and Wallace, B. A. DICHROWEB, an online server for protein secondary structure analyses from circular dichroism spectroscopic data. Nuc. Ac. Res. 2004, 32, W668-673.

219. Campbell, B. N. Jr., Araiso, T., Reinisch, L., Yue, K. T., Hager, L. P. A kinetic study of the binding of carbon monoxide to ferrous chloroperoxidase. Biochem. 1982, 21, 4343-4349.

220. Lambeir, A. M., Heremans, K., Dunford, H. B. High-pressure effect on the equilibrium and kinetics of cyanide binding to chloroperoxidase. Biophys. Chem. 1983, 18, 195-201.

221. Gallati, H. Horseradish-peroxidase - study of the kinetics and the determination of optimal reaction conditions, using hydrogen-peroxide and 2,2-azino-bis-3ethylbenzthiazoline-6-sulfonic acid (ABTS) as substrates. J. Clin. Chem. Clin. Biochem. 1979, 17, 1-7.

222. Hager, L. P., Morris, D. R., Brown, F. S., Eberwein, H. Chloroperoxidase. II. Utilization of halogen anions. J. Biol. Chem. 1966, 241, 1769-1777.

223. Banci, L., Bertini, I., Turano, P., Ferrer, J. C., Mauk,G. Comparative ${ }^{1} \mathrm{H}$ NMR study of ferric low-spin cytochrome c peroxidase and horseradish peroxidase. Inorg. Chem. 1991, 30, 4510-4516.

224. Richards, F. M. Areas, volumes, packing and protein structure. Annu. Rev. Biophys. Bioeng. 1977, 6, 151-176.

225. Baumann, G., Frommel, C., Sander, C. Polarity as a criterion in protein design. Prot. Eng. 1989, 2, 329-334.

226. Pardillo, A. D., Morozov, A. N., Chatfield, D. C. Proximal pocket hydrogen bonds significantly influence the mechanism of chloroperoxidase Compound I formation. J. Phys. Chem. 2015, 119, 12590-12602.

227. Nwamba, C. O. and Chilaka, F. C. A proposed significance of the $\delta$ region and its implications in the mechanism of cooperativity in hemoglobin. Med. Hypotheses Res. 2010, 6, 25-35.

228. Blanke, S. R., Martinis, S. A., Sligar, S. G., Hager, L. P. Probing the heme iron coordination structure of alkaline chloroperoxidase. Biochemistry 1996, 35, 
$14537-14543$.

229. Danehy, J. P. and Parameswaran, K. N. Acidic dissociation constants of thiols. J. Chem. Eng. Data 1968, 13, 386-389.

230. Bethe, H. Splitting of terms in crystals. Ann. Phys. 1929, 3, 133-206.

231. Kramers, H. A. A general theoty of paramagnetic rotation in crystals. Proc. Kon. Nederland. Akad. Wetenschappen. 1930, 33, 959-972.

232. Van Vleck, J. H. Valence strength and the magnetism of complex salts. J. Chem . Phys. 1935, 3, 807-813.

233. Banci, L. Structural properties of peroxidases. J. Biotechnol. 1997, 53, 253-263.

234. Dawson, J. H. and Sono, M. Cytochrome P-450 and chloroperoxidase: thiolateligated heme enzymes. Spectroscopic determination of their active-site structures and mechanistic implications of thiolate ligation. Chem. Rev. 1987, 87, 1255-1276.

235. Harris, D. L., Loew,G. H. Theoretical investigation of the proton assisted pathway to formation of cytochrome P450 Compound I. J. Am. Chem. Soc. 1998, 120, 8941-8948.

236. Dey, A., Jiang, Y., Ortiz de Montellano, P., Hodgson, K. O., Hedman, B., Solomon, E. I. S K-edge XAS and DFT calculations on cytochrome P450: covalent and ionic contributions to the cysteine - Fe bond and their contribution to reactivity. J. Am. Chem. Soc. 2009, 131, 7869-7878.

237. Harris, R. Z., Newmyer, S. L., Ortiz de Montellano, P. R. Horseradish peroxidase-catalyzed two-electron oxidations. Oxidation of iodide, thioanisoles, and phenols at distinct sites. J. Biol. Chem. 1993, 268, 1637-1645.

238. Kelly, S. M. and Price, N. C. The use of circular dichroism in the investigation of protein structure and function. Curr. Prot. Pept. Sci. 2000, 1, 349-384.

239. Casella, L., Gullotti, M., Poli, S., Bonfa, M., Ferrari, R. P., Marchesini, A. Spectroscopic and binding studies on the stereoselective interaction of tyrosine with horseradish peroxidase and lactoperoxidase. Biochem. J. 1991, 279, 245250.

240. Hill, A. V. The possible effects of the aggregation of the molecules of haemoglobin on its dissociation curves. J. Physiol. 1910, 40, 389-403. 
241. Kay, E., Strickland, H., Billups, C. Near ultraviolet circular dichroism and absorption of chicken ovomucoid and acetylated derivatives at 297 and $77^{\circ} \mathrm{K}$. J. Biol. Chem. 1974, 249, 797-802.

242. De Villiers, K. A., Kaschula, C. H., Egan, T. J., Marques, H. M. Speciation and structure of ferriprotoporphyrin IX in aqueous solution: Spectroscopic and diffusion measurements demonstrate dimerization, but not $\mu$-oxo dimer formation. J. Biol. Inorg. Chem. 2007, 12, 101-117.

243. Lambeir, A. M., Dunford, H. B., Pickard, M. A. Kinetics of cyanide binding to chloroperoxidase in the presence of nitrate: detection of the influence of a heme-linked acid group by shift in the apparent pKa value. J. Inorg. Biochem. 1983, 19, 291-300.

244. Poulos, T. L. The role of the proximal ligand in heme enzymes. J. Biol. Inorg. Chem. 1996, 1, 356-359.

245. Dawson, J. H., Holm. R. H., Trudell, J. R., Barth, G., Linder, R. E., Bunnenberg, E., Djerassi, C., Tang, S. C. Magnetic circular dichroism studies. 43. Oxidized cytochrome P-450. Magnetic circular dichroism evidence for thiolate ligation in the substrate-bound form. Implications for the catalytic mechanism. J. Am. Chem. Soc. 1976, 98, 3707-3709.

246. Ogliaro, F., de Visser, S. P., Shaik, S. The 'push' effect of the thiolate ligand in cytochrome P450: a theoretical gauging. J. Inorg. Biochem. 2002, 91, 554-567.

247. Crane, B. R., Arvai, A. S., Gachhui, R., Wu, C., Ghosh, D. K., Getzoff, E.D., Stuehr, D. J., Tainer, J. A. The structure of nitric oxide synthase oxygenase domain and inhibitor complexes. Science 1997, 278, 425-431.

248. Ueno, T., Kousumi, Y., Yoshizawa-Kumagaye, K., Nakajima, K., Ueyama, N., Okamura, T., Nakamura, A. Role of $\alpha$-helix conformation cooperating with $\mathrm{NH} \cdots \mathrm{S}$ hydrogen bond in the active site of cytochrome P-450 and chloroperoxidase: synthesis and properties of [MIII(OEP)(Cys-helical peptide)] (M = Fe and Ga). J. Am. Chem. Soc., 1998, 120, 12264-12273.

249. Filizola, M. and Loew, G. H. Probing the role of protein environment in Compound I formation of chloroperoxidase. J. Am. Chem. Soc. 2000, 122, 3599-3605.

250. Tanaka, M., Morimoto, A., Ishimori, K., Morishima, I. Structure-activity relation of horseradish peroxidases as studied with mutations at heme distal and proximal sites. Pure Appl. Chem. 1998, 70, 911-916. 
251. Sinclair. R., Hallam, H., Chen, M., Chance, B., Powers, L. Active site structure in cytochrome $c$ peroxidase and myoglobin mutants: effects of altered hydrogen bonding to the proximal histidine. Biochemistry 1996, 35, 15120-15128.

252. Carpena, X., Vidossich, P., Schroettner, K., Calisto, B. M., Banerjee, S., Stampler, J., Soudi, M., Furtmu"ller, P. G., Rovira, C., Fita, I., Obinger, C. Essential role of proximal Histidine-Asparagine interaction in mammalian peroxidases. J. Biol. Chem. 2009, 284, 25929-25937.

253. Stellwagen, E. Haem exposure as the determinate of oxidation-reduction potential of haem proteins. Nature 1978, 275, 73-74.

254. Furtmüller, P. G., Zederbauer, M., Jantschko, W., Helm, J., Bogner, M., Jakopitsch, C., Obinger, C. Active site structure and catalytic mechanisms of human peroxidases. Arch. Biochem. Biophys. 2006, 445, 199-213. 


\section{APPENDIX}

Figure A1. Primers used for construction of C29H/C79H and C29H/C79H/C87H CPO (His mutation is shown in bold).

Coding strain - $\mathrm{C} 29 \mathrm{H} / \mathrm{C} 79 \mathrm{H}$ primer 1 (C29H pCPO was used as a template)

5'- GTCGTCCACGAGTACGTTACTGGCTCCGACTGTGGTGACAGC - 3'

Non-coding strain - C29H/C79H primer 2

5’- AGCCAGTAACGTACTCGTGGACGACGAAGGTGTTGTCAGAGC - 3’

Coding strain - C29H/C79H/C87H primer 1 (C29H/C79H pCPO was used as a template) 5’- CTCCGACCATGGTGACAGCCTTGTCAACCTGACTC - 3’

Non-coding strain - C29H/C79H/C87H primer 2

5’- GTCACCATGGTCGGAGCCAGTAACGTACTCGTG - 3’

Figure A2. Nucleotide sequence of full-length WT CPO gene.

ATGTTCTCCAAGGTCCTTCCCTTCGTGGGAGCGGTTGCCGCCСTCCСTCACTC CGTCCGTCAGGAGCCTGGCTCCGGCATTGGCTACCCATACGACAACAACACC CTGCCATATGTCGCCCCAGGTCCTACCGACTCTCGTGCTCCTTGCCCAGCTCT GAACGCTCTTGCCAACCACGGTTACATTCCTCACGATGGCCGTGCCATCAGC AGGGAGACCCTCCAGAACGCTTTCCTCAACCACATGGGTATTGCCAACTCCG TCATTGAGCTTGCTCTGACCAACGCCTTCGTCGTCTGCGAGTACGTTACTGGC TCCGACTGTGGTGACAGCCTTGTCAACCTGACTCTGCTCGCCGAGCCCCACGC TTTCGAGCACGACCACTCCTTCTCCCGCAAGGATTACAAGCAGGGTGTCGCC AACTCCAACGACTTCATCGACAACAGGAACTTCGATGCCGAGACCTTCCAGA CCTCTCTGGATGTCGTTGCAGGCAAGACCCACTTCGACTATGCCGACATGAA CGAGATCCGCCTTCAGCGCGAGTCCCTCTCCAACGAGCTTGACTTCCCCGGTT GGTTCACCGAGTCCAAGCCAATCCAGAACGTCGAGTCTGGCTTCATCTTCGCC CTTGTCTCTGACTTCAACCTGCCCGACAACGATGAGAACCCTCTGGTTCGCAT TGACTGGTGGAAGTACTGGTTCACCAACGAGTCCTTCCCATACCACCTCGGCT 
GGCACCCCCCGTCTCCAGCCAGGGAGATCGAGTTCGTCACCTCCGCCTCCTCC GCTGTCCTGGCTGCCTCTGTCACCTCTACTCCATCTTCCCTTCCATCCGGTGCC ATCGGCCCAGGTGCCGAGGCTGTCCСTCTCTCCTTCGCCTCCACCATGACCCC ATTCCTCCTCGCCACCAATGCTCCTTACTACGCCCAGGACCCAACTCTCGGCC CCAACGACAAGCGTGAGGCTGCCCCAGCTGCCACCACCTCCATGGCCGTCTT CAAGAACCCATACCTCGAGGCCATTGGCACCCAGGACATCAAGAACCAGCA GGCTTACGTCAGCTCCAAGGCTGCTGCCATGGCCTCTGCCATGGCCGCCAAC AAGGCCCGCAACCTTTAA

Figure A3. Amino acid sequence of WT CPO including signal peptide (1-20) and propeptide (322-373).

1 MFSKVLPFVG AVAALPHSVR QEPGSGIGYP YDNNTLPYVA PGPTDSRAPC 51 PALNALANHG YIPHDGRAIS RETLQNAFLN HMGIANSVIE LALTNAFVVC 101 EYVTGSDCGD SLVNLTLLAE PHAFEHDHSF SRKDYKQGVA NSNDFIDNRN 151 FDAETFQTSL DVVAGKTHFD YADMNEIRLQ RESLSNELDF PGWFTESKPI 201 QNVESGFIFA LVSDFNLPDN DENPLVRIDW WKYWFTNESF PYHLGWHPPS 251 PAREIEFVTS ASSAVLAASV TSTPSSLPSG AIGPGAEAVP LSFASTMTPF 301 LLATNAPYYA QDPTLGPNDK REAAPAATTS MAVFKNPYLE AIGTQDIKNQ 351 QAYVSSKAAA MASAMAANKA RNL 
VITA

\section{ELENA SHERSHER}

$2000-2003$

$2007-2011$

$2010-2011$

$2013-2014$

$2011-2014$

$2011-2016$

2012

2013

2013
Linguistic Studies

Institute of International Relations and Law

Tolyatti, Russia

Bachelor of Arts in Chemistry

Minor in Biology

Florida International University

Miami, Florida, USA

Teaching Assistant

Florida International University

Miami, Florida, USA

Presidential Fellowship

Florida International University

Miami, Florida, USA

Doctoral Student (biochemistry)

Florida International University

Miami, Florida, USA

Biomedical Research Initiative Student Research Award Funded through the MBRS RISE

Florida International University

Miami, Florida, USA

Best Graduate Student Oral Presentation

15th Annual Biomedical and Comparative Immunology

Symposium

Miami, Florida, USA

\section{PUBLICATIONS AND PRESENTATIONS}

Shersher, Elena; Wang, Xiaotang. "The Role of Hydrogen Bonding between Arg26 and Asn37 in Chloroperoxidase Catalysis,” 250th ACS National Meeting \& Exposition, Boston, MA, United States, August 16-20, 2015 (2015), BIOL-185.

Shersher, Elena; Bolhassani, Andrew; Wang, Xiaotang. "The Role of Proximal Thiolate in Chloroperoxidase Catalysis.” 249th ACS National Meeting \& Exposition, Denver, CO, United States, March 22-26, 2015 (2015), BIOL-200. 
Shersher, Elena; Wang, Xiaotang. "Characterization of Bovine Prion Protein Interactions with Wogonin, a Potential Antiprion Compound," 16 ${ }^{\text {th }}$ Annual Biomedical and Comparative Immunology Symposium, Miami, FL, United States, February 13-14, 2014 (oral presentation).

Shersher, Elena; Wang, Xiaotang. "Prion Protein in Transmissible Spongiform Encephalopathies and Identification of Antiprion Compounds," $15^{\text {th }}$ Annual Biomedical and Comparative Immunology Symposium, Miami, FL, United States, March 7-8, 2013 (oral presentation). 